\title{
USING PASSIVE SAMPLERS TO ASSESS BIOAVAILABILITY, TOXICITY, AND REACTIVITY OF HYDROPHOBIC ORGANIC CHEMICALS (HOCS)
}

\author{
By \\ Alexandra Patricia Tcaciuc \\ B.S., Chemistry, Environmental Engineering Science \\ Massachusetts Institute of Technology, 2009 \\ Submitted in partial fulfillment of the requirements for the degree of \\ Doctor of Philosophy \\ at the \\ MASSACHUSETTS INSTITUTE OF TECHNOLOGY \\ and the \\ WOODS HOLE OCEANOGRAPHIC INSTITUTION
}

June 2015

(C)2015 Alexandra Patricia Tcaciuc. All rights reserved.

The author hereby grants to MIT and WHOI permission to reproduce and to

distribute publicly paper and electronic copies of this thesis document in whole or in part in any medium now known or hereafter created.

Signature of Author

Department of Civil and Environmental Engineering, MIT \&

Department of Marine Chemistry and Geochemistry, WHOI

April 30, 2015

Certified by

Philip M. Gschwend

Ford Professor of Civil and Environmental Engineering, MIT

Thesis Supervisor

Accepted by

Elizabeth B. Kujawinski

Associate Scientist of Marine Chemistry \& Geochemistry, WHOI

Chair, Joint Committee for Chemical Oceanography

Accepted by

Heidi Nepf

Donald and Martha Harleman Professor of Civil and Environmental Engineering, MIT

Chair, Graduate Program Committee 


\title{
Using Passive Samplers to Assess Bioavailability, Toxicity, and Reactivity of Hydrophobic Organic Chemicals (HOCs)
}

\author{
by \\ Alexandra Patricia Tcaciuc
}

Submitted to the Department of Civil and Environmental Engineering (MIT) and the Academic Programs Office (WHOI) on April 30th, 2015 in partial fulfillment of the requirements for the degree of Doctor of Philosophy in the field of Environmental Chemistry and Geochemistry

\begin{abstract}
Hydrophobic organic chemicals (HOCs) are a class of environmental contaminants responsible for numerous acute and chronic health effects in humans and wildlife. This thesis illustrates three applications of polyethylene (PE) passive sampling, which enhance our toolbox for estimating environmental hazards associated with HOCs.

First, we present a methodology that can be used to estimate the bioaccumulation potential of numerous organic chemicals based on passive sampling and comprehensive two dimensional gas chromatography $(\mathrm{GC} \times \mathrm{GC})$. Using GC $\times \mathrm{GC}$ retention times, we show that lipid-water and samplerwater partition coefficients can be estimated within a factor of 2 and 3, respectively. The method was then applied to estimate body burdens of various HOCs in benthic organisms from GC $\times$ GC analysis of PE equilibrated with contaminated sediment. Empirical observations of accumulation in the Nereis virens polychaete were in good agreement with PE-based predictions for PCBs, but were lower by at least an order of magnitude for other classes of HOCs (such as PAHs) presumably due to metabolism.

Second, we applied the same methodology to a set of contaminated sediments and estimated the cumulative baseline toxicity associated with environmental mixtures of HOCs. The predictions were compared against empirical measurements of baseline toxicity using the water flea Daphnia magna. The estimated total body burdens of HOCs were in good agreement with measured toxicity, with toxicity occurring at body burdens larger than $30 \mathrm{mg} / \mathrm{g}_{\text {lipid }}$. In contrast, the toxicity estimated based on priority pollutants severely underestimated the observed toxicity, emphasizing the importance of cumulative effects.

Lastly, to advance our understanding of the processes that affect passive sampling results in situ (when they are operating away from equilibrium), a mathematical model was developed for reactive chemicals transferring between PE and sediment beds. The reaction diffusion model was used to infer in situ degradation rates of dichlorodiphenyltrichloroethane (DDT), which in the sediments of a freshwater lake were found to be between 0.09 and $0.9 \mathrm{~d}^{-1}$. A second mathematical model describing the kinetics of exchange between passive samplers and water was also developed, which can be used in both field (infinite baths) and laboratory (finite baths) conditions.
\end{abstract}

Thesis Supervisor: Philip M. Gschwend Title: Ford Professor of Civil and Environmental Engineering, MIT 


\section{ACKNOWLEDGMENTS}

This research has been supported by funding from the MIT Sea Grant College Program (grant numbers NA060AR4170019 and NA100AR4170086); CICEET, the Cooperative Institute for Coastal and Estuarine Environmental Technology (award number 015553-001); ENI S.p.A through the MIT Energy Initiative (contract numbers 5200003626 and 5210000541); Exponent, Inc. as part of ESTCP project ER-201216 (S15-0551); ESTCP, the Environmental Security Technology Certification Program (W912HQ-09-C-0008); SEA Engineering as part of a US EPA project (agreement dated 8/13/13 under prime award EP-S9-08-04); the MIT Presidential Fellowship and the Martin Family Society of Fellows for Sustainability at MIT. A special thanks also to the Ippen Travel Fund (MIT) which has enabled me to travel to conferences and share my work with the research community.

First and foremost, I would like to thank my adviser, Phil Gschwend, who inspired me to look at chemistry from a different perspective. I am grateful for his tremendous support along the way, for everything that he has taught me, and for his patience and guidance. I thank him for holding my work to a high scientific and writing standard, and for encouraging me to pursue independent research ideas.

I could not have hoped for a better thesis committee. In the past three years, Chris Reddy and Ann Tarrant, along with Phil, have provided valuable feedback and constant encouragements, and shared their resources and knowledge with me. Chris has graciously allowed me to use his instruments many times. A big thank you as well to Ben van Mooy for chairing my defense and also allowing me to use his lab.

I would also like to thank the numerous people who made this work possible starting with John MacFarlane who is the most awesome, most patient and most generous research technician any graduate (or undergraduate) student could ever hope for! I owe a lot of my laboratory skills to his patient teaching and I've benefited from his support on numerous occasions when research was, well, research...

An extensive group of people helped acquire, prepare, ship and analyze the various samples in this thesis. At ENI, Raffaella Borrelli, Pietro Cesti, Fabio Vago, and Alessandro Oldani, and Luciano Zaninetta (Syndial) were instrumental to fabricating, deploying and retrieving the passive samplers in Lake Maggiore. I also want to thank Robert Burgess (EPA) for generously sharing his expertise with setting up bioaccumulation tests, and Guilherme Lotufo (US ACE) for providing the Lauritzen Channel sediment samples, and collaborators Scott Carr and Marion Nipper (Texas A\&M) whose work I had the opportunity to build on.

I have also been very fortunate to have an amazing group of lab mates, Loretta Fernandez, Dave Griffith, Debra Hausladen, Da Deng, Dave Kuo, Desiree Plata, Jordon Hemingway, Elizabeth Finn (Follett), Jennifer Apell, Dan Prendergast, and Zhijiang Lu, who have helped in countless ways from teaching me various techniques, to reading drafts, shuttling samples to WHOI and just making 48412 an awesome place to work. A special thanks to Jennifer Apell for bringing in some extra enthusiasm and drive when mine was running low, for proofreading the numerous drafts of Chapter 4 and for letting me bounce ideas off of her (and vice versa). Also, I could not have done 
half the work I did in the past year without the help of two hardworking and talented undergraduate students, Josefin Betsholtz and Lauren Rotkovitz.

I also like to thank my second academic family in Christopher Reddy's lab at WHOI, and in particular Bob Nelson, who always found time to squeeze my samples in the GC $\times$ GC queue, as well as Karin Lemkau, Catherine Carmichael, and Christoph Aeppli who showed me how to use various instruments and made me feel welcome in Fye on every occasion.

I have been fortunate to be a part of both the Joint Program and the Parsons Lab communities and I am grateful for all the brilliant people that I have met. In Parsons Lab, I want to thank the members of Supergroup (Irene Hu, Kyle Delwiche, Sarah Jane White, Schuyler Senft Grupp, Anthony Carrasquillo, Kelly Daumit, James Hunter, Ben Scandella, Mason Stahl, Alison Hoyt, Amy Muller, Matt Orosz and more) - I have learned a lot from you guys and my presentations would not have been half as good without your feedback. Thank you to my cubicle neighbors and friends, Teresa Yamana and Jessie Berta-Thompson, who fueled this thesis with plenty of coffee breaks. I've also benefited from the scientific insight of numerous faculty at MIT including Charles Harvey and Heidi Nepf. I am grateful for the friendship and advice of many JP students (Britta Voss, Jill McDermott, Kim Popendorf, Jess Fitzsimmons, Chris Follett and more). A special thank you as well to the members of the JCCO who have helped me navigate the Joint Program - Liz Kujawinski, Mark Kurz and Bernhard Peucker-Ehrenbrink.

Although their hard work is not mentioned here (but in their own theses) I am grateful to the three graduate student mentors (Loretta Fernandez, Cristina Cismasu and Charu Varadharajan) who took me under their wings when I was an inexperienced undergraduate and let me mess with their experiments so that I could learn!

I am also grateful to the wonderful staff in Parsons - Vicki Murphy, whose door is always open and whose office always has chocolate, Jim Long, who helps us all keep our head above the water and Sheila Frankel, who among many other things, found me my first summer job. A special thanks also to the academic administrators and support staff for being so understanding and supportive to the graduate students, at WHOI - Lea Fraser, Marsha Armando, Tricia Gebbie and Julia Westwater, Valerie Caron, Sheila Clifford and at MIT - Kiley Clapper, Kris Kipp and Ronni Schwartz. I'm sure we all ask the same questions over and over again and you bear with us!

My family and friends have helped me every step of the way and I wouldn't have made it this far without their constant encouragement and support. Special thanks to my parents and grandparents for raising me, to my great-grandmother for instilling in me the gift of patience, and to my mother for always believing in me (much more than I did). Thank you also to my oldest and dearest friend Irina, for always putting a smile on my face, but also to Alexandra, Ryan, Tristan, Erica and many more, for being awesome friends!

Last, but not least, my deepest gratitude goes out to my husband Mishu who shared this journey with me, reminded me to eat, sleep and take vacations, and put up with me (or the absence of me) in the past few months. He has been an endless source of understanding, advice and kindness and I am looking forward to our next adventures together. 


\section{TABLE OF CONTENTS}

Chapter 1. Hydrophobic organic chemicals (HOCs) and passive sampling ............ 15

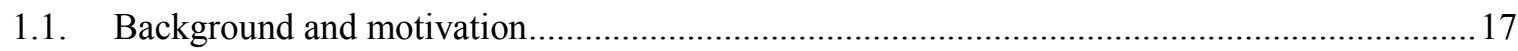

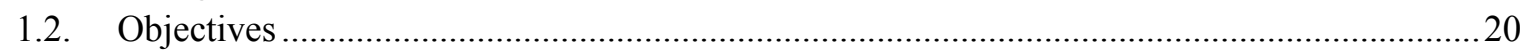

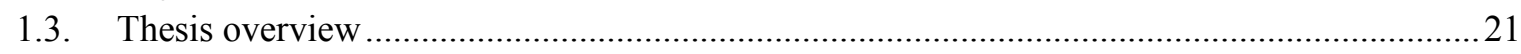

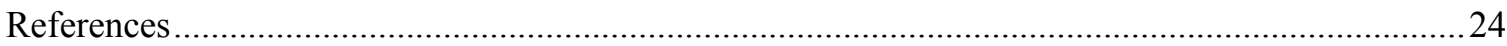

\section{Chapter 2 Estimating phospholipid membrane-water partition coefficients using} comprehensive two-dimensional gas chromatography .................................................227

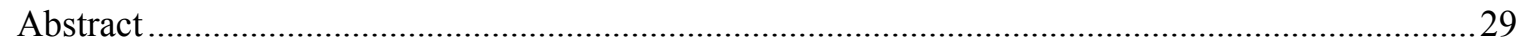

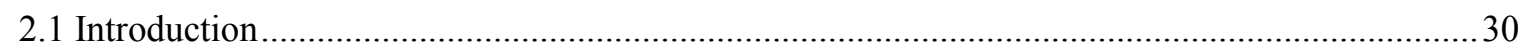

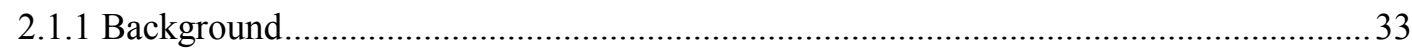

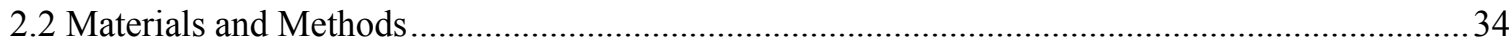

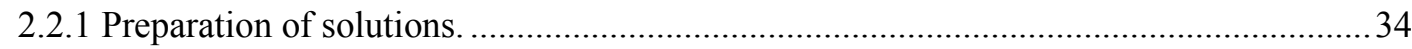

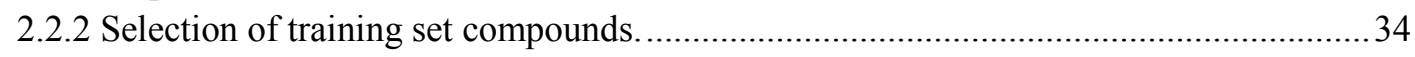

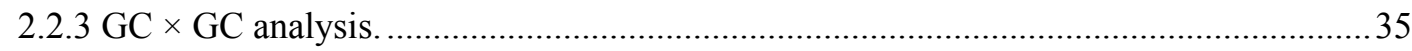

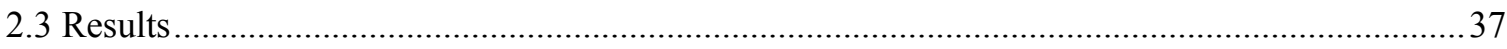

2.3.1 GC $\times$ GC system check and use of retention times to estimate $\log K_{O W}$ values ..............37

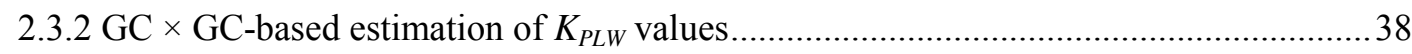

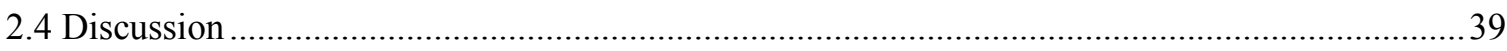

2.4.1 Comparison of $\mathrm{GC} \times \mathrm{GC}$ method versus ppLFER and $\log K_{O W}$ approaches ...................39

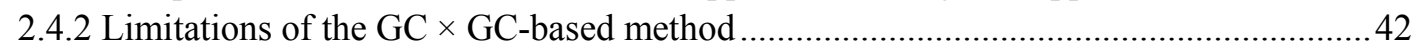

2.4.3 Applications of the GC $\times$ GC-based method: estimating $K_{P L W}$ values for new compounds

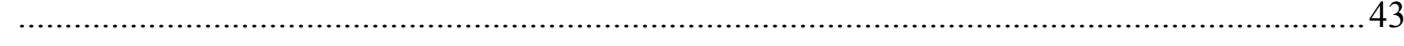

2.4.4 Applications of the GC $\times$ GC-based method: estimating baseline narcosis risks........... 44

2.4.5 Applications of the $\mathrm{GC} \times \mathrm{GC}$-based method: estimating bioaccumulation of mixtures 45

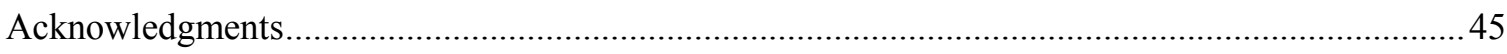

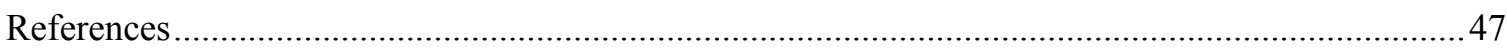

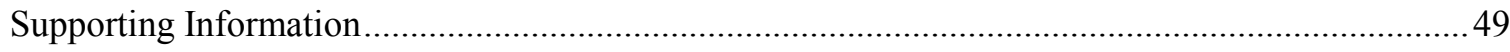

\section{Chapter 3.Evaluating bioaccumulation potential of HOC mixtures using GC $\times$ GC} and passive sampling ............................................................................................................................. 61

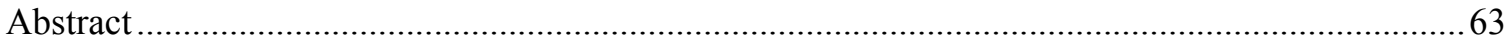

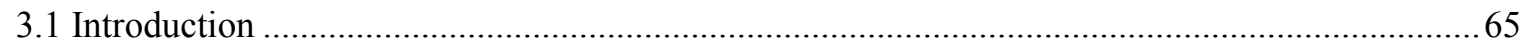

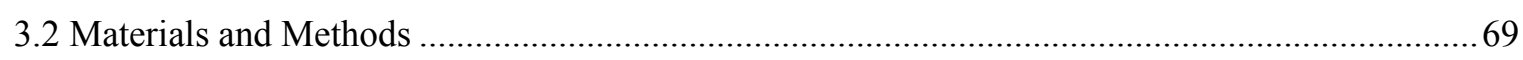

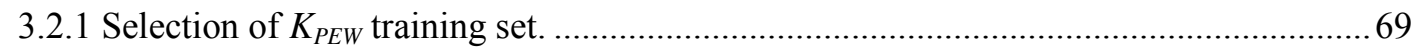

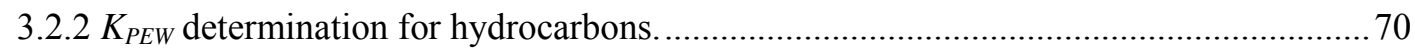

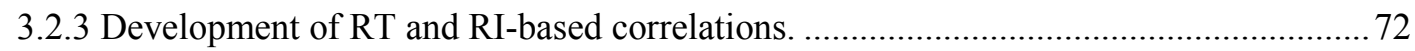

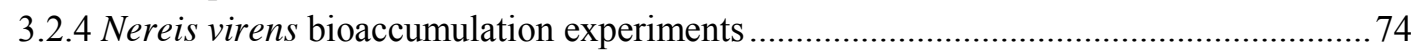

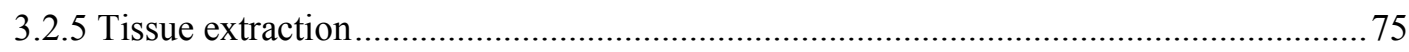

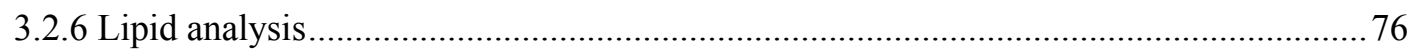

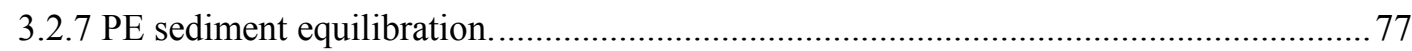




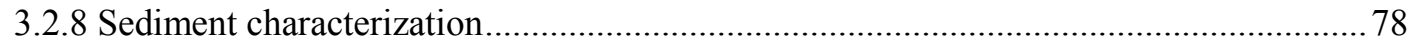

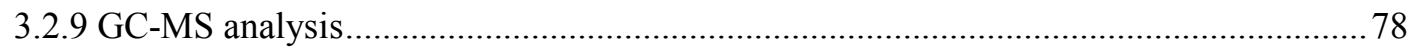

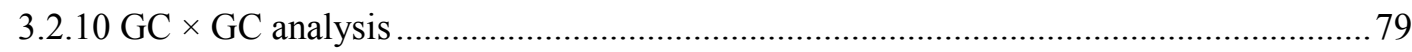

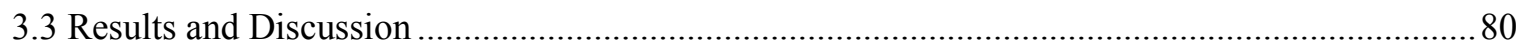

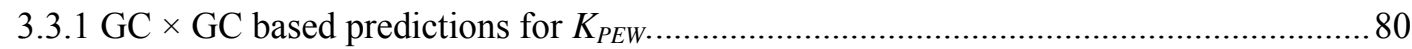

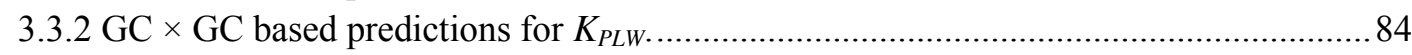

3.3.3 GC $\times$ GC-based predictions of lipid/PE differential accumulation of HOCs .................. 86

3.3.4 Accumulation of HOCs in Nereis virens and GC $\times$ GC based predictions ..................... 89

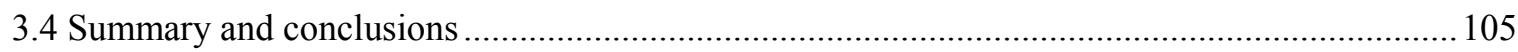

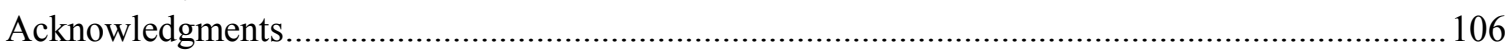

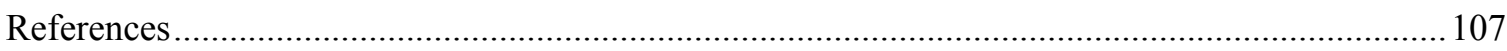

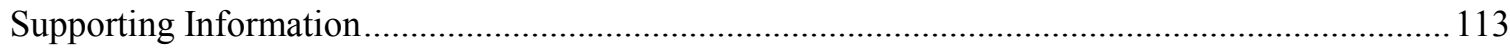

\section{Chapter 4. Modeling the transport of organic chemicals between polyethylene} passive samplers and water in finite and infinite bath conditions ........................... 139

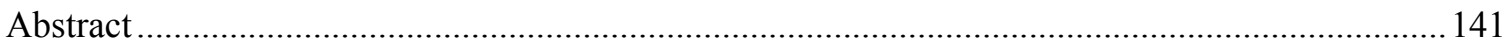

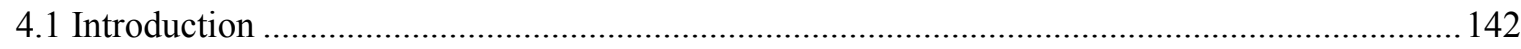

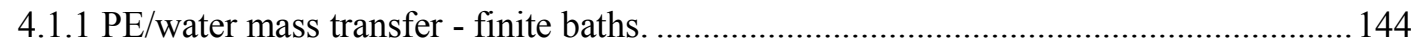

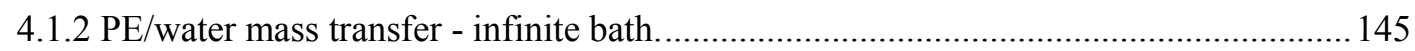

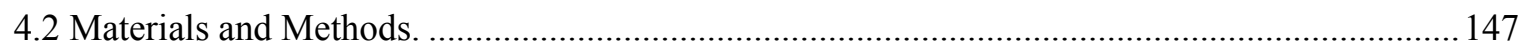

4.2.1 Phenanthrene and pyrene uptake at variable stirring speeds. .................................... 147

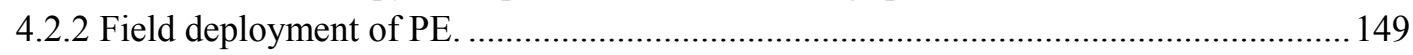

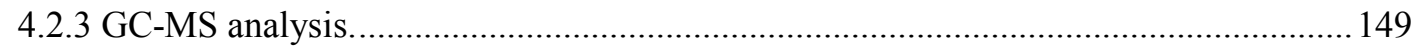

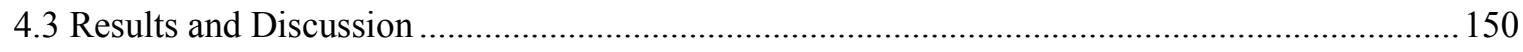

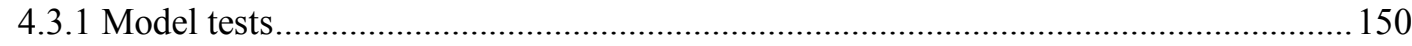

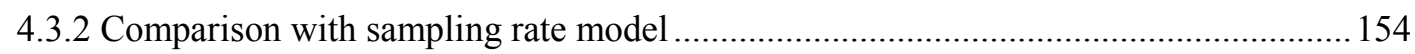

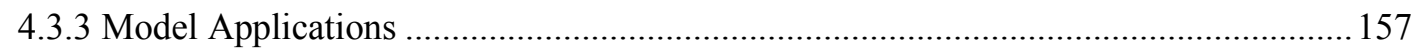

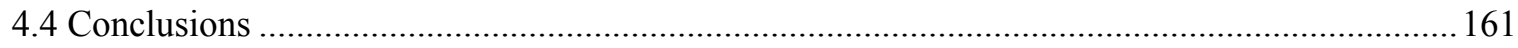

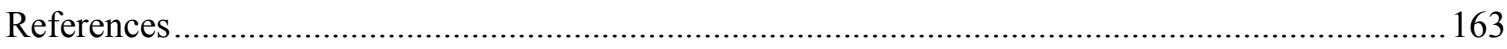

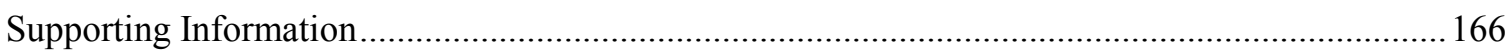

\section{Chapter 5. Characterizing baseline toxicity of HOC mixtures with GC $\times$ GC and passive dosing ............................................................................................................................................. 191}

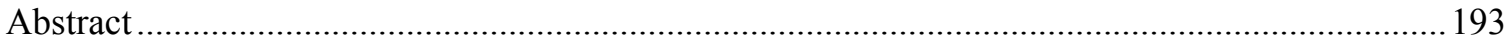

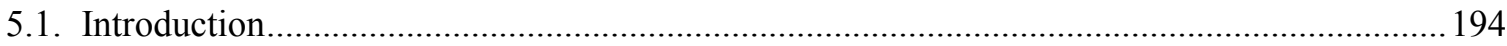

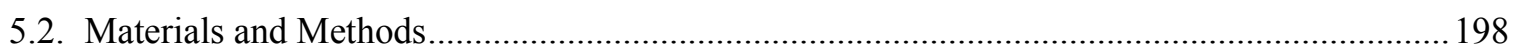

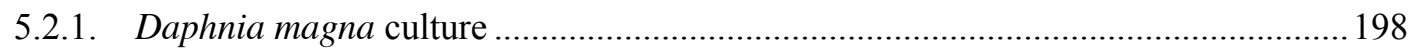

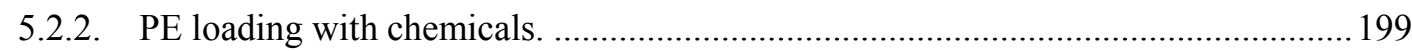

5.2.3. Characterization of PE-water equilibration timescales in static conditions............... 199

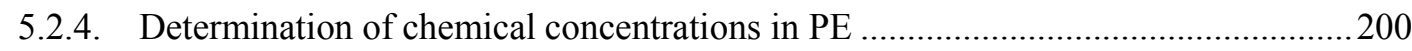

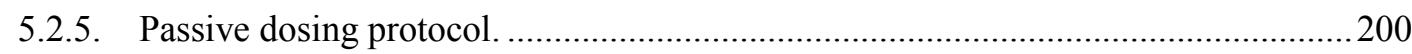




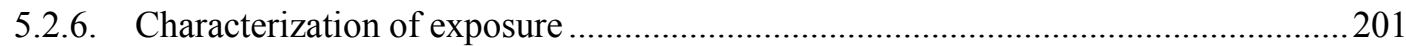

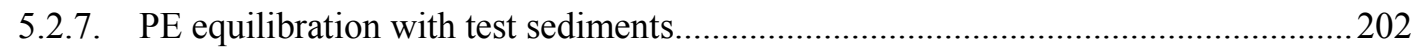

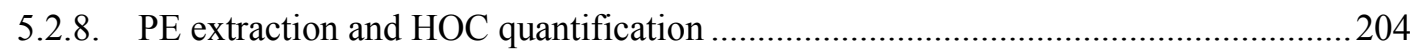

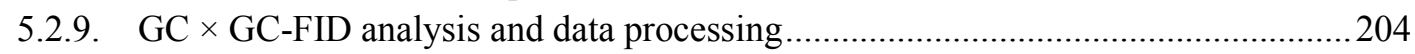

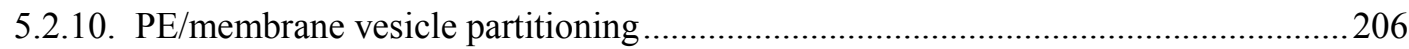

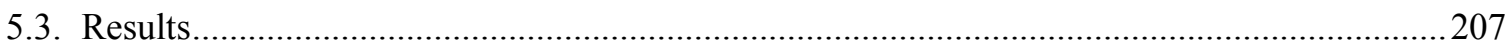

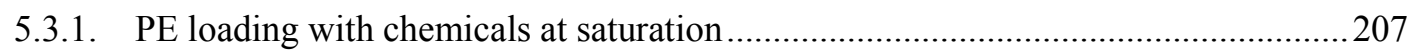

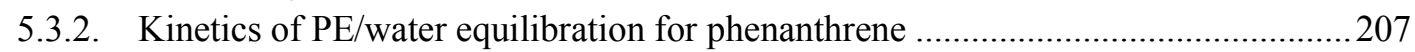

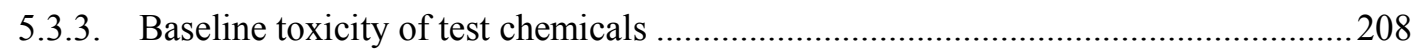

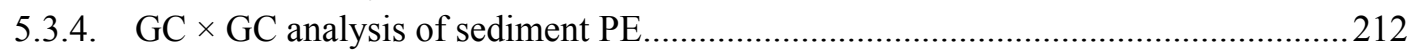

5.3.5. Comparison between empirical and GC $\times$ GC-based estimations of toxicity ..........2 216

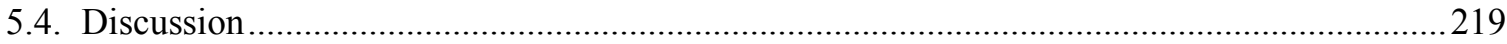

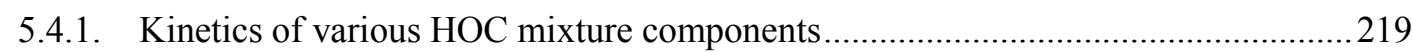

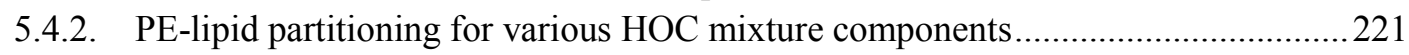

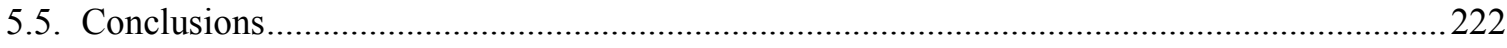

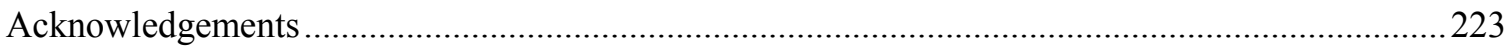

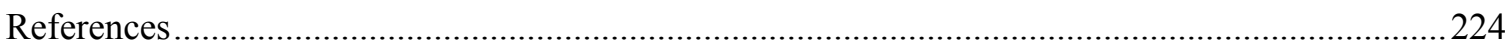

\section{Chapter 6. Measuring in-situ degradation rates of DDT with passive sampling . 229}

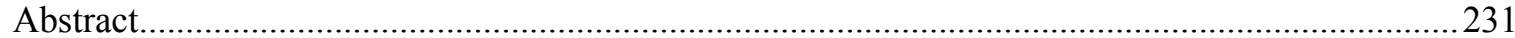

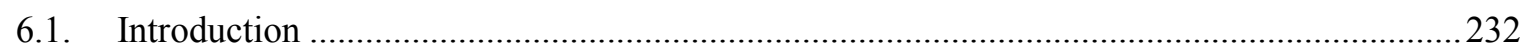

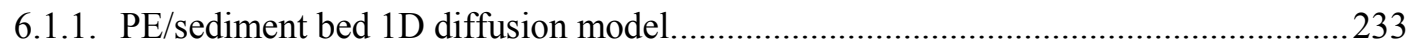

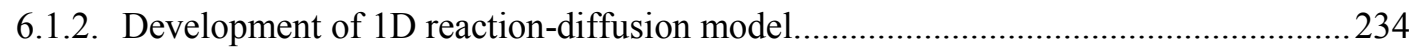

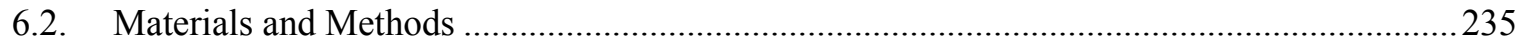

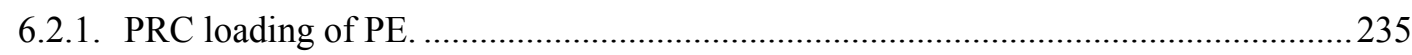

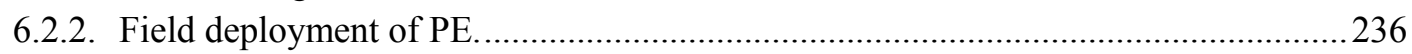

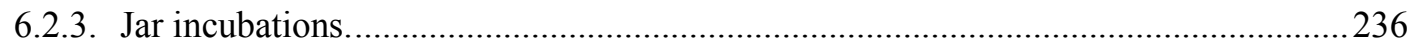

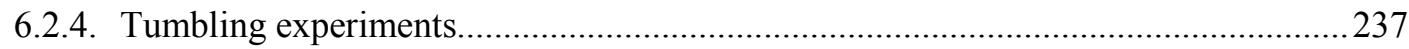

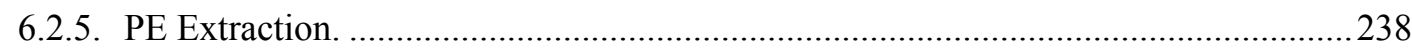

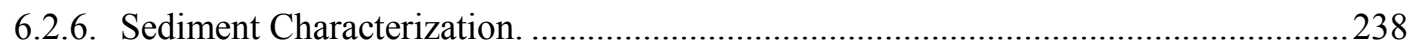

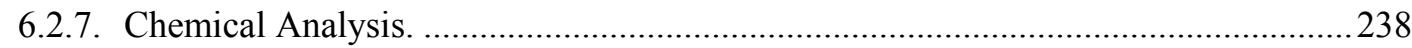

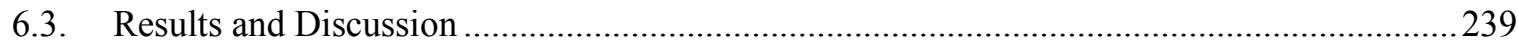

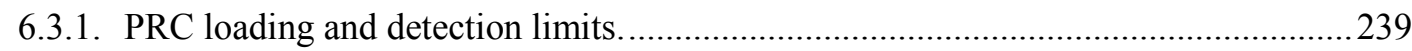

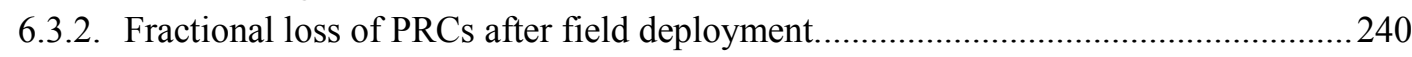

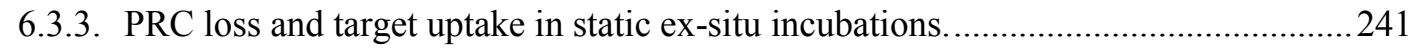

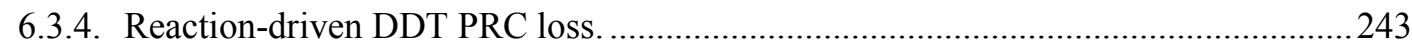

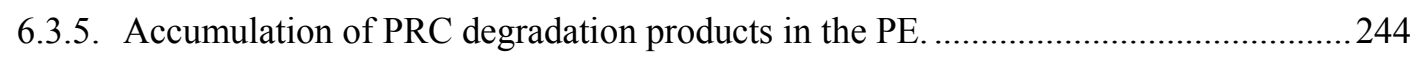

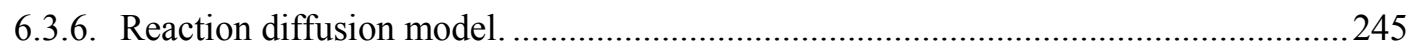

6.3.7. Implications for interpretation of PRCs and measuring reactive compounds...........249

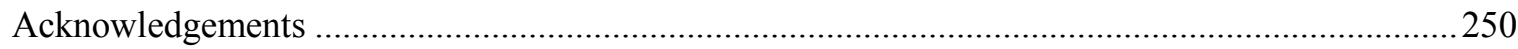

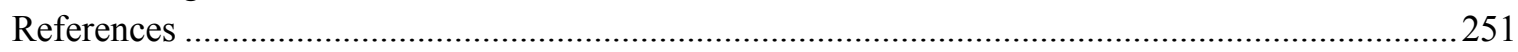

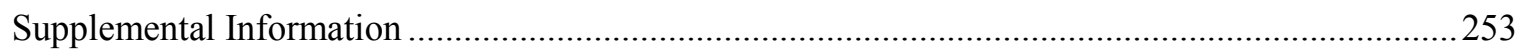




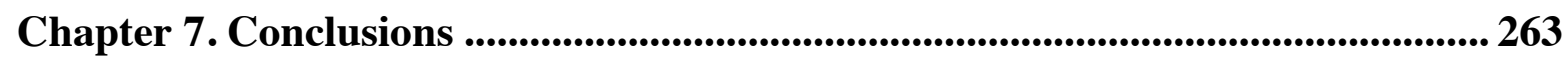

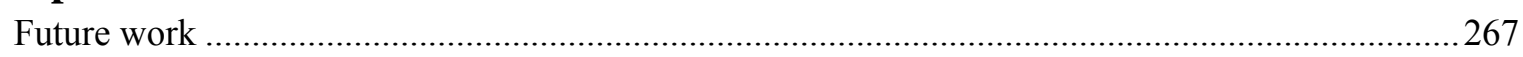

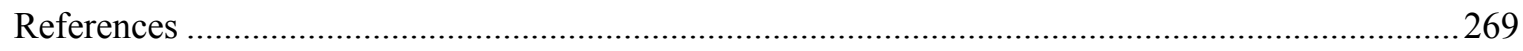




\section{LIST OF FIGURES}

\section{Chapter 2 Estimating phospholipid membrane-water partition coefficients using comprehensive two-dimensional gas chromatography

Figure 2.1 Examples of a triglyceride storage lipid and a membrane phospholipid ..............32

Figure 2.2 $K_{\mathrm{PLW}}$ from $\mathrm{GC} \times \mathrm{GC}, K_{\mathrm{OW}}$ and Abraham polyparameter model ..........................40

Figure $2.3 K_{\mathrm{PLW}}$ of $\mathrm{PCBs}$ and $\mathrm{OCPs}$ from $\mathrm{GC} \times \mathrm{GC}$ and Abraham polyparameter model....44

\section{Chapter 3.Evaluating bioaccumulation potential of HOC mixtures using GC $\times$ GC and passive sampling .............................................................................................................. 61}

Figure 3.1 Schematic of relationships between concentrations in various compartments with equilibrium partitioning or toxico-kinetic approaches

Figure 3.2 Nereis virens lipid contents .78

Figure 3.3 Measured and $\mathrm{GC} \times \mathrm{GC}$-derived $K_{P E W}$ values using retention times and retention indices for PAHs, PCBs, hydrocarbons (HCs) and organochlorine pesticides (OCPs .83

Figure 3.4 Correlation between $\log K_{P E W}$ and the first dimension retention vector. .84

Figure 3.5 Comparison between retention time and retention indices methods for evaluating $\log K_{P L W}$ across the $\mathrm{GC} \times \mathrm{GC}$ retention space.

Figure 3.6 Contour plots of the ratio of $K_{P L W} / K_{P E W}$, in $\log _{10}$ space, against retention indices

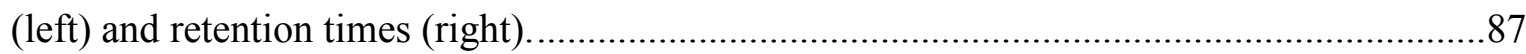

Figure 3.7 PCB concentrations measured in blank and exposed worms...............................92

Figure 3.8 Comparison of PCBs concentrations in PE and corresponding worm tissue........94

Figure 3.9 Ratio of PAH concentrations measured in lipid and in PE .................................97

Figure 3.10 Fraction of 4,4'-DDE, 4,4'-DDD and 4,4'-DDT of total 4,4'-DDX in the sediment, $N$. virens tissue and $\mathrm{PE}$ for $\mathrm{C} 400$ and $\mathrm{C} 1800$ sediments ...................................100

Figure 3.11 Ratio of concentrations in worms and PE for various DDXs ...........................102

Figure $3.12 \mathrm{GC} \times \mathrm{GC}-\mathrm{FID}$ chromatograms of PE and worms incubated with Island End sediment.

Chapter 4. Modeling the transport of organic chemicals between polyethylene passive samplers and water in finite and infinite bath conditions 
Figure 4.1 Phenanthrene and pyrene uptake by PE at different stirring speeds 151

Figure 4.2 Best fit boundary layer thicknesses as a function of stirring speed for phenanthrene and pyrene

Figure 4.3 Model fit results for finite bath sorption experiments illustrated for select PCB and DDTs

Figure 4.4 Illustration of membrane controlled, mixed membrane-WBL controlled and WBL controlled mass transfer between PE and and an infinite water bath

Figure 4.5 Measured fractions of PRCs left in field deployed $25 \mu \mathrm{m}$ and $10 \mu \mathrm{m}$ PE after 10 day and 30 day deployments in the water column of Lake Maggiore.

\section{Chapter 5. Characterizing baseline toxicity of HOC mixtures with GC $\times$ GC and passive dosing}

Figure 5.1 Approach to equilibrium of phenanthrene desorbing from a $100 \mu \mathrm{m}$ PE in static conditions. .208

Figure 5.2 Dose response curves for phenanthrene and 1,3,5-trichlorobenzene in $48 \mathrm{~h}$ Daphnia magna immobilization tests.

Figure 5.3 Daphnia magna immobilization against concentration in the lipid 211

Figure 5.4. GC $\times$ GC-FID chromatograms of PE extracts and model-estimated PL chromatograms for Island End, C1800 and Tabbs Bay 8 test sediments.....

Figure 5.5 Distribution of priority chemical classes measured in PE equilibrated with each test sediment and predicted in the lipid.

Figure 5.6. Comparison between \% Daphnia magna immobilization observed using 1 day and 3 day pre-equilibration times.

Figure 5.7. Observations of \% Daphnia magna immobilization versus estimated body burdens from $\mathrm{GC} \times \mathrm{GC}$-based analysis of sediment-equilibrated PE

Figure 5.8 Measured DMPC-PE partition coefficients and GC $\times$ GC-estimated PL-PE partition coefficients for phenanthrene, $1,3,5-\mathrm{TCB}, n \mathrm{C} 14$ benzene and octadecane.

\section{Chapter 6. Measuring in-situ degradation rates of DDT with passive sampling ....229}

Figure 6.1 Fractional PRC remaining in PE as a function of time in PE deployment in sediment at various depth horizons....

Figure 6.2 Fraction of PRC remaining and fraction of target compound for static incubation of PE in F2, CARM and LM sediment for 4,4'-DDE and 4,4'-DDD. .241 
Figure 6.3 Fraction of ${ }^{13} \mathrm{C} 2,4$ '-DDT PRC remaining in PE and measured accumulation of the degradation product ${ }^{13} \mathrm{C} 2,4^{\prime}$-DDD after deployment in sediment at various depth

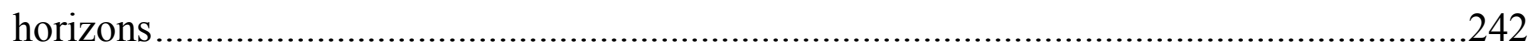

Figure 6.4 Fraction of ${ }^{13} \mathrm{C} 2,4^{\prime}$-DDT remaining in $\mathrm{PE}$ and measured as its degradation product ${ }^{13} \mathrm{C} 2,4^{\prime}$-DDD as a function of time after incubation in CARM, LM, and F2

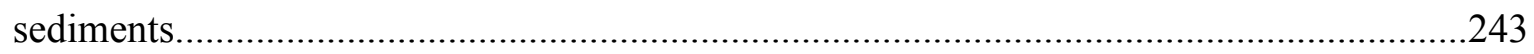

Figure 6.5 Fraction of DDT PRC remaining after 10 and 30 day deployments at various depths into the sediment. .245

Figure 6.6 Model fits for the fraction of ${ }^{13} \mathrm{C} 2,4^{\prime}$-DDT remaining in PE as a function of time after incubation in CARM, LM and F2 246

Figure 6.7 Accumulation of target compounds and release of PRCs for the non-reactive compound, DDE and for the reactive compound, DDT . .248 


\section{LIST OF TABLES}

\section{Chapter 2 Estimating phospholipid membrane-water partition coefficients using comprehensive two-dimensional gas chromatography}

Table 2.1. Experimental and predicted $\log K_{P L W}$ using three methods: $\mathrm{GC} \times \mathrm{GC}$,

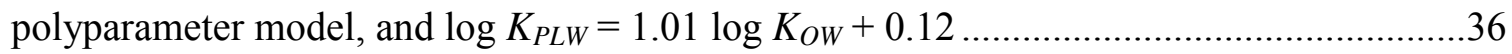

Table 2.2. Poly-parameter model coefficients for evaluating retention behavior on the GC $\times$ GC stationary phases, as well as for calculating $\log K_{O W}, \log K_{P L W}$ and $\log K_{\text {olive oil/water } \ldots . . . .42}$

\section{Chapter 3.Evaluating bioaccumulation potential of HOC mixtures using GC $\times$ GC} and passive sampling

Table 3.1. Regression lines for evaluating partition coefficients $K_{P E W}$ and $K_{P L W}$ from retention time and retention indices

Table 3.2. Concentration of total PAHs, total quantified PCBs and total DDXs measured in sediments, worm tissue and tumbled PE in the four test sediments, 303.1, C400, C1800 and Island End, along with the corresponding $\mathrm{f}_{\mathrm{OC}}$ and $\mathrm{f}_{\mathrm{BC}}$ measured for each sediment.

Chapter 5. Characterizing baseline toxicity of HOC mixtures with GC $\times$ GC and passive dosing

Table 5.1. Tabulated properties of various test compounds and measured concentrations at saturation in PE and in pure water equilibrated with PE at $21^{\circ} \mathrm{C}$

Table 5.2. Total concentration of HOCs calculated based on GC $\times$ GC-FID chromatograms of $\mathrm{PE}$ equilibrated with various sediments, and corresponding model-estimated total concentration of HOCs in membrane lipids.

\section{Chapter 6. Measuring in-situ degradation rates of DDT with passive sampling ....229}

Table 6.1. Log $K_{d}$ values determined from PE/sediment tumbling experiments $\left(K_{d, T}\right)$, and from fits of diffusion model to PRC loss from field deployed $\left(K_{d, F}\right)$ and PRC loss from laboratory incubated PE $\left(K_{d, L}\right)$ in various sediments collected from Lake Maggiore. 


\section{Chapter 1.}

Hydrophobic organic chemicals (HOCs) and passive sampling 


\subsection{Background and motivation}

Use of synthetic chemicals has become an integral part of our society. We routinely use chemicals or chemical products in industrial manufacturing, agriculture, water treatment, transportation and more. But this extensive use has also led to unintended consequences. A recent survey found that as much as $11 \%$ of the surveyed coastline contained sediments that were posing health threats to humans and wildlife. ${ }^{1}$ Adding to this, is the growing concern over the large volumes of synthetic compounds (300 million tons) produced annually and the impacts that their release could have in the environment. ${ }^{2}$ For many of these chemicals, the potential for adverse effects is unknown, and the unintended negative effects are often only realized after the chemical is already in use (e.g., the case for dichlorodiphenyltrichloroethane (DDT), triclosan). Even less is known about the effects of the mixtures of these chemicals. There is thus a clear need for better understanding the fate and impacts of chemicals in the environment, which can help us make better decisions about which chemicals we use and how we manage our natural resources.

Many toxic organic contaminants fall under the broad category of hydrophobic organic chemicals (HOCs), and numerous HOCs are at the same time, bioaccumulative, persistent and toxic to aquatic life and to humans. HOCs include legacy pollutants such as polychlorinated biphenyls (PCBs), which were banned in 1979, as well as contaminants of current or emerging concern, such as polybrominated diphenyl ethers (PBDEs) and perfluorinated compounds (PFCs). For many of these compounds, the strong bonds that impart their thermal and chemical stability, and make them excellent industrial chemicals (e.g., PFCs are used in non stick coatings, PBDEs are flame retardants), also make them resistant to environmental degradation. Most HOCs mentioned thus far have been linked to chronic and acute toxicity effects, which threaten the health of ecosystems (e.g., DDT impairs bird reproduction ${ }^{3}$ ) as well as humans (e.g., PBDE exposure linked to neurodevelopmental toxicity ${ }^{4}$ ). Contamination of aquatic systems with HOCs can affect not only the health of communities but also their economic livelihood and food security. In many places across the US, contamination with DDTs and PCBs has lead to fishing advisories or fishing bans. ${ }^{5}$ Lastly, for persistent HOCs that undergo long-range transport, the exposed population is not limited to the vicinity of chemical "hot spots", but includes remote locations as far as the Arctic Ocean. ${ }^{6,7}$ 
Remediation of contaminated sites is often focused on sediments because sediments tend to be the largest aquatic reservoir of HOCs. While traditional risk assessment techniques rely on bulk measurements of sediment concentration, there is a growing consensus that risk assessment should be based on freely dissolved concentrations of chemicals. ${ }^{8}$ The freely dissolved concentration is a metric of the contaminant's chemical activity (or fugacity), which in turn governs its tendency to be mobilized and transported into the overlying water or taken up by organisms. Sediments can act as a sink for contaminants due to the presence of highly sorptive phases like organic carbon and black carbon, which sequester and reduce the chemical activity of HOCs in sediments. ${ }^{9}, 10$ But sediments can also act as a source of contamination through sediment to water diffusive fluxes ${ }^{11}$ or through migration of contaminants up the food webs. ${ }^{12}$ Whether the sediment beds act as a sink or source depends on difference in chemical activities between the sediment and the overlying water. Various studies have shown that porewater concentrations (or freely dissolved concentrations) were better predictors of bioaccumulation, ${ }^{13}$ toxicity, ${ }^{14}$ and sediment-to-water fluxes ${ }^{15}$ compared to estimates based on bulk sediment concentrations. In theory, estimations of porewater concentrations from bulk sediment concentration via the sediment-water partition coefficient $\left(K_{d}\right)$ should yield the same result as direct porewater measurements, but in practice, the large uncertainty associated with $K_{d}$ renders this approach inaccurate. ${ }^{16}$

Advances in passive sampling in recent years have improved the ease and reliability of measuring freely dissolved concentration of HOCs in sediments. With passive sampling, dissolved concentrations are measured based on the accumulation of chemicals in a polymer membrane such as polyethylene (PE). The PE accumulates chemicals according to their fugacity or chemical activity, and with known values of polyethylene-water partition coefficients, the concentrations of chemicals in PE can be translated in the corresponding freely dissolved concentrations in the porewater (or in the water in the case of water column sampling). In contrast, direct porewater measurements can be biased high by inclusion of HOCs sorbed to colloids and dissolved organic carbon. In addition, adjusting the measured concentration to the freely dissolved value, requires quantification of the organic carbon concentration in the sample, as well as assumptions about the sorptive capacity of the dissolved organic carbon. Passive sampling techniques also have the advantage of improved detection limits over direct porewater measurements. For example, assuming an average detection limit of $10 \mathrm{pg} / \mu \mathrm{L}$ would allow us to 
easily measure concentrations of $10 \mathrm{pg} / \mathrm{L}$ in the porewater (assuming an extract volume of 0.1 $\mathrm{mL}$ and sampler mass of $0.1 \mathrm{~g}$; this leads to detection limits in the sampler of $10 \mathrm{ng} / \mathrm{g}$, which for a compound with a sampler-water partition coefficient of $10^{6}$ corresponds to an aqueous concentration of $10 \mathrm{pg} / \mathrm{L}$ ). Instead, the detection limit for a direct porewater measurement would be higher $(\sim 1 \mathrm{ng} / \mathrm{L}$, for the same extract volume of $0.1 \mathrm{~mL}$ and assuming $1 \mathrm{~L}$ of porewater is extracted) and the porewater measurement would require considerably more effort (i.e., $1 \mathrm{~L}$ of porewater requires processing of $\sim 4 \mathrm{~L}$ of sediment). Passive sampling involves a simpler analytical procedure, which makes it a suitable technique for assessing heterogeneity of contamination in field sites.

Baseline or narcosis toxicity is one of the mechanisms through which HOCs can affect the health of exposed organisms. Yet, after more than 100 years of narcosis research, we still do not fully understand the biochemical mechanism behind this toxicity. ${ }^{17}$ We know from studies of lethal body burdens, that narcosis occurs when body burdens reach $34-400 \mu \mathrm{mol} / \mathrm{g}_{\text {lipid }},{ }^{18-20}$ but several researchers have raised the concern that there are multiple types of lipid, with different affinities for chemicals ${ }^{21-23}$ and only the accumulation of chemicals inside the membrane lipids is likely to elicit a biological response. ${ }^{22}$ Thus, a better criteria needs to be established based on membrane lipid chemical burdens. Lastly, since organisms are regularly exposed to mixtures of narcotic chemicals at various concentrations, some models have been developed for evaluating narcosis toxicity of mixtures. They are based on the assumption that narcosis effects are additive and they require knowledge about the identity and the octanol-water partition constant $\left(K_{\mathrm{OW}}\right)$ of all the mixture components, which can be difficult to obtain in some cases. One example is the unresolved complex mixture (UCM) associated with petroleum contamination, for which methods of estimating narcosis toxicity are still being developed. ${ }^{19,}{ }^{24}$ Narcosis toxicity due to petroleum hydrocarbons is a concern in marine environments, given the large inputs of oil to the sea from a wide array of sources (natural seeps, extraction, and transportation) and its toxicity to marine biota. ${ }^{25}$

Even though the presence of HOC mixtures is widespread in the environment, the focus of current regulatory standards, as well as that of passive sampling exercises thus far, has been on individual contaminants. ${ }^{26,27}$ Cumulative toxicity is a concern, however, given that approximately $60 \%$ of the industrial chemicals entering the aquatic environment, have been linked to narcosis toxicity, ${ }^{28}$ and that many chemical products such as fuels or lubricants are 
often mixtures as well (e.g., aroclors, PBDE technical mixtures). Furthermore, recent studies ${ }^{29}$ have shown that some HOCs (in particular PAHs such as anthracene, benzo[a]pyrene, which have limited chemical activity at saturation due to large melting points $>200{ }^{\circ} \mathrm{C}$ and high entropies of fusion), exhibit limited toxicity when tested individually in Daphnia magna immobilization tests, but they do cause toxicity when tested as a mixture in the same immobilization assay. Thus, it is noteworthy that there may be HOCs (e.g., high melting point PAHs but potentially other HOCs with large entropies of fusion), for which individual dose response curves cannot be derived, and the calculation of cumulative effects based on toxicity units $^{30}$ (ratio of concentration to LC50) may not accurately represent the cumulative toxicity of a mixture.

In evaluating the toxicity of environmental mixtures, one must consider both the composition of the mixture as well as the dissolved concentrations of its components (i.e., their availability to partition into membrane lipids). For example, numerous studies have tried to understand the toxicity of UCMs associated with petroleum contamination, by measuring toxicity of individual chemicals $^{31}$ or the toxicity of the compounds present in an exhaustive solvent extract. ${ }^{24,32}$ But these approaches do not account for the bioavailability of mixture components in the environment. On the other hand, passive sampling studies have been undertaken for evaluating contamination with priority pollutants (Greenberg et al. ${ }^{33}$ and references within) but very few targeted HOC mixtures, either in terms of bioaccumulation ${ }^{34}$ or toxicity. ${ }^{35}$

\subsection{Objectives}

The main objective of this thesis was to expand the use of passive samplers from measurement of individual chemicals towards characterization of bioaccumulation and toxicity of mixtures of HOCs. To this end, equilibrium passive sampling was combined with comprehensive two-dimensional gas chromatography $(\mathrm{GC} \times \mathrm{GC})$ to help us characterize the composition and properties of environmental mixtures. Recent studies have devised methods for using $\mathrm{GC} \times \mathrm{GC}$ retention times for the calculation of numerous environmentally relevant properties such as aqueous solubilities, vapor pressures and $K_{O W} \cdot{ }^{36-38}$ In this work we developed similar methods for evaluating polyethylene-water $\left(K_{P E W}\right)$ and the phospholipid membrane-water $\left(K_{P L W}\right)$ partition coefficients from $\mathrm{GC} \times \mathrm{GC}$ retention times, which can be used to calculate the corresponding concentration of a chemical in the lipids of an exposed organism, for any given 
concentration in the passive sampler. The advantages of using chromatography for evaluating partition coefficients is that we can apply this method without needing to identify all the mixture components.

A second objective of this work was to advance our understanding of passive sampling exercises, particularly when passive samplers are used in non-equilibrium conditions or for targeting reactive compounds (i.e., chemicals that degrade on timescales that smaller or comparable to passive sampling deployment timescales which are on the order of days to months). For in situ passive sampler deployments, most HOCs do not have enough time to reach equilibrium concentrations in the sampler, ${ }^{39}$ and the measured concentration in the sampler has to be adjusted upwards by the extent of disequilibrium. This adjustment is usually done based on the measured loss during the deployment time of performance reference compounds (PRCs), which are pre-loaded into the sampler before deployment. For in situ deployments in sediments, it is believed that the PRC loss is controlled by diffusion through the sediment bed, but less is known about what controls the PRC loss when samplers are deployed in water or when targeting reactive chemicals. ${ }^{40,41}$ Better understanding the processes that impact the PRC loss can help us (1) gain confidence in the passive sampling results and (2) provide useful biogeochemical information about the sampled environment. For example, existing mathematical models ${ }^{42,} 43$ relate the PRC loss to the sediment $K_{d}$, which is difficult to estimate or measure experimentally, but particularly useful in assessing the bioavailability and mobility of contaminants out of sediment beds. The working hypothesis for reactive chemicals (i.e. degrading on timescales of days to months) was that in situ passive sampling with PRCs coupled with a reaction diffusion model could provide estimates about in situ degradation rates.

\subsection{Thesis overview}

In Chapter 2, we present a method for estimation of $K_{P L W}$ values for nonpolar HOCs from $\mathrm{GC} \times \mathrm{GC}$ retention times, which can be used to evaluate baseline toxicity potential of mixtures, as well as membrane-water partition coefficients for numerous HOCs. In Chapter 3 we develop a similar relationship for $K_{P E W}$ and we investigate whether the combination of equilibrium passive sampling and $\mathrm{GC} \times \mathrm{GC}$-based equilibrium partition model could provide accurate predictions of bioaccumulation for a range of HOCs. To this end, a series of contaminated sediments were selected which were previously collected from (1) Lauritzen Channel, Richmond, CA, a 
Superfund site in San Francisco Bay contaminated with DDT from on-shore pesticide packing and shipping activities until $1966^{44}$ and (2) Island End, Chelsea, MA a hydrocarbon contaminated site in Boston Harbor, situated next to an coal tar processing facility, operational between $1890 \mathrm{~s}$ to late $1950 \mathrm{~s}^{45}$. The sediments collected from these two sites were used in bioaccumulation experiments with the polychaete Nereis virens. The contamination patterns present in the test sediments allowed us to compare the measured accumulation in the tissues of Nereis virens of a range of HOCs including PAHs, PCBs, DDTs, and UCM hydrocarbons, against estimated body burdens of the same chemicals using passive samplers and GC $\times$ GC. Furthermore, the test sediments also had a wide range of organic and black carbon contents, which allowed us to explore the impact of sorptive phases on bioavailability of contaminants, as measured both in the PE and in the worms.

Chapter 4 is dedicated to understanding the mass transfer between PE passive samplers in water in both finite (laboratory) and infinite (field) conditions. The Laplace space mathematical model characterizing the PE/water exchange was derived by applying Fick's second law to the diffusion of chemicals through the polymer membrane and an aqueous boundary layer. In addition the model was applied towards understanding the PE-water kinetics in finite bath systems to infer whether PE could be used as a dosing phase in toxicity assays. Lastly, the model was employed for the interpretation of data from passive samplers deployed in Lake Maggiore, a subalpine freshwater lake situated at the border of Italy and Switzerland. Lake Maggiore has been subject to DDT contamination due to the waste disposal of a DDT manufacturing plant situated on the River Toce, which flows into Lake Maggiore. Field activities at this site were directed towards characterization of dissolved concentrations using passive sampling, of DDT and its degradation products DDE and DDD in both sediments and water column. Chapter 4 will focus on the data from the water column samplers, whereas Chapter 6 will focus on the passive sampler deployments in the sediment beds.

In Chapter 5, we investigate the link between $\mathrm{GC} \times \mathrm{GC}$-based predictions of body burdens, which were used to estimate baseline toxicity, and empirical observations of toxicity from Daphnia magna immobilization tests. To measure the toxicity of HOCs mixtures present in various sediments, a novel PE-based passive dosing protocol was designed and implemented in which the polymer membrane equilibrated with contaminated sediment was used to reproduce the composition and chemical activity of environmental mixtures in test vials with Daphnia 
magna. Compared to traditional sediment toxicity tests, passive dosing was chosen because it was more likely to reflect the toxicity of HOCs (as opposed to other water quality issues or inorganic contaminants) in the sediment. The toxicity associated with sediment-equilibrated PE was measured for sediments collected from Island End and Lauritzen Channel sites described above, as well as Tabbs Bay, TX, a site contaminated with oil field produced water. The set of test sediments were chosen to reflect different levels of contamination as well as different distribution of HOC mixtures (various combination of priority pollutants and unresolved complex mixtures). As such, sediments collected from Island End were used because they contained both UCM hydrocarbons as well as PAH priority pollutants. The sediments from Lauritzen Channel had high concentrations of DDT (and degradation products DDE and DDD), as well as a late-eluting hydrocarbon signature. In contrast, the sediments from Tabbs Bay were predominantly contaminated with an early eluting hydrocarbon mixture, and contained very little PAH contamination.

Finally, a reaction-diffusion transport model is introduced in Chapter 6. The model was applied to measured PRC loss from field-deployed PE in the sediment bed of Lake Maggiore (discussed above) and used to derive the magnitude of in situ degradation rates of DDT. Given the history of the DDT pollution at Lake Maggiore, the site was suitable for the study of DDT degradation processes, and measurements of degradation rates are relevant both to any future application of PRC-loaded passive samplers at the site, as well as for assessing the long term fate of the DDT in the lake. A summary of major findings and potential future research avenues will be presented in Chapter 7. 


\section{References}

1. US Environmental Protection Agency National Coastal Conditions Report II; EPA620/R-03/002; Washington, DC, USA 2004.

2. Schwarzenbach, R. P.; Escher, B. I.; Fenner, K.; Hofstetter, T. B.; Johnson, C. A.; von Gunten, U.; Wehrli, B., The challenge of micropollutants in aquatic systems. Science 2006, 313, (5790), 1072-1077.

3. Cooke, A. S., Shell thinning in avian eggs by environmental pollutants. Environmental Pollution (1970) 1973, 4, (2), 85-152.

4. $\quad$ Eskenazi, B.; Chevrier, J.; Rauch, S. A.; Kogut, K.; Harley, K. G.; Johnson, C.; Trujillo, C.; Sjodin, A.; Bradman, A., In utero and childhood polybrominated diphenyl ether (PBDE) exposures and neurodevelopment in the CHAMACOS study. Environ Health Perspect 2013, 121, (2), 257-62.

5. US Environmental Protection Agency National Listing of Fish Advisories. http://fishadvisoryonline.epa.gov/Advisories.aspx

6. de Wit, C. A.; Herzke, D.; Vorkamp, K., Brominated flame retardants in the Arctic environment--trends and new candidates. Sci Total Environ 2010, 408, (15), 2885-918.

7. Dewailly, E.; Nantel, A.; Weber, J. P.; Meyer, F., High levels of PCBs in breast milk of Inuit women from Arctic Quebec. Bull Environ Contam Toxicol 1989, 43, (5), 641-6.

8. $\quad$ Mayer, P.; Parkerton, T. F.; Adams, R. G.; Cargill, J. G.; Gan, J.; Gouin, T.; Gschwend, P. M.; Hawthorne, S. B.; Helm, P.; Witt, G.; You, J.; Escher, B. I., Passive sampling methods for contaminated sediments: scientific rationale supporting use of freely dissolved concentrations. Integr Environ Assess Manag 2014, 10, (2), 197-209.

9. Lohmann, R.; MacFarlane, J. K.; Gschwend, P. M., Importance of black carbon to sorption of native PAHs, PCBs, and PCDDs in Boston and New York, Harbor sediments. Environ Sci Technol 2005, 39, (1), 141-148.

10. Koelmans, A. A.; Jonker, M. T.; Cornelissen, G.; Bucheli, T. D.; Van Noort, P. C.; Gustafsson, 0., Black carbon: the reverse of its dark side. Chemosphere 2006, 63, (3), 36577.

11. Fernandez, L. A.; Lao, W.; Maruya, K. A.; Burgess, R. M., Calculating the diffusive flux of persistent organic pollutants between sediments and the water column on the Palos Verdes shelf superfund site using polymeric passive samplers. Environ Sci Technol 2014, 48, (7), 3925-34.

12. Khairy, M. A.; Weinstein, M. P.; Lohmann, R., Trophodynamic behavior of hydrophobic organic contaminants in the aquatic food web of a tidal river. Environ Sci Technol 2014, 48, (21), 12533-42.

13. Kraaij, R.; Seinen, W.; Tolls, J., Direct evidence of sequestration in sediments affecting the bioavailability of hydrophobic organic chemicals to benthic deposit-feeders. Environ Sci Technol 2002, 36, (16), 3525-3529.

14. Kreitinger, J. P.; Neuhauser, E. F.; Doherty, F. G.; Hawthorne, S. B., Greatly reduced bioavailability and toxicity of polycyclic aromatic hydrocarbons to Hyalella azteca in sediments from manufactured-gas plant sites. Environ Toxicol Chem 2007, 26, (6), 11461157.

15. Liu, H. H.; Bao, L. J.; Zhang, K.; Xu, S. P.; Wu, F. C.; Zeng, E. Y., Novel passive sampling device for measuring sediment-water diffusion fluxes of hydrophobic organic chemicals. Environ Sci Technol 2013, 47, (17), 9866-73. 
16. Accardi-Dey, A.; Gschwend, P. M., Reinterpreting literature sorption data considering both absorption into organic carbon and adsorption onto black carbon. Environ Sci Technol 2003, 37, (1), 99-106.

17. Sandberg, W. S.; Dubois, B. W.; Miller, K. M., Lipid membrane and ligand-gated ion channels in general anesthetic action. In Current Topics in Membranes. , Academic Press: 1999; Vol. 48, pp 311-350.

18. McCarty, L. S.; Mackay, D.; Smith, A. D.; Ozburn, G. W.; Dixon, D. G., Residue-based interpretation of toxicity and bioconcentration QSARs from aquatic bioassays - neutral narcotic organics. Environ Toxicol Chem 1992, 11, (7), 917-930.

19. Di Toro, D. M.; McGrath, J. A.; Hansen, D. J., Technical basis for narcotic chemicals and polycyclic aromatic hydrocarbon criteria. I. Water and tissue. Environ Toxicol Chem 2000, 19, (8), 1951-1970.

20. McGrath, J. A.; Parkerton, T. E.; Hellweger, F. L.; Di Toro, D. M., Validation of the narcosis target lipid model for petroleum products: Gasoline as a case study. Environ Toxicol Chem 2005, 24, (9), 2382-2394.

21. Jabusch, T. W.; Swackhamer, D. L., Partitioning of polychlorinated biphenyls in octanol/water, triolein/water, and membrane/water systems. Chemosphere 2005, 60, (9), 1270-8.

22. Sandermann, H., Jr., Differential lipid affinity of xenobiotics and natural compounds. Febs Lett 2003, 554, (1-2), 165-8.

23. Endo, S.; Escher, B. I.; Goss, K. U., Capacities of membrane lipids to accumulate neutral organic chemicals. Environ Sci Technol 2011, 45, (14), 5912-21.

24. Mao, D.; Lookman, R.; Van De Weghe, H.; Weltens, R.; Vanermen, G.; De Brucker, N.; Diels, L., Estimation of ecotoxicity of petroleum hydrocarbon mixtures in soil based on HPLC-GCXGC analysis. Chemosphere 2009, 77, (11), 1508-13.

25. National Research Council, Oil in the Sea III: Inputs, Fates, and Effects. The National Academies Press: Washington, D.C., 2003.

26. Altenburger, R.; Greco, W. R., Extrapolation concepts for dealing with multiple contamination in environmental risk assessment. Integr Environ Assess Manag 2009, 5, (1), 62-8.

27. Landrum, P. F.; Chapman, P. M.; Neff, J.; Page, D. S., Evaluating the aquatic toxicity of complex organic chemical mixtures: lessons learned from polycyclic aromatic hydrocarbon and petroleum hydrocarbon case studies. Integr Environ Assess Manag 2012, 8, (2), 217-30. 28. Veith, G. D.; Call, D. J.; Brooke, L. T., Structure-toxicity relationships for the fathead minnow, Pimephales promelas: narcotic industrial chemicals. Can J Fish Aquat Sci 1983, 40, (6), 743-748.

29. Smith, K. E.; Schmidt, S. N.; Dom, N.; Blust, R.; Holmstrup, M.; Mayer, P., Baseline toxic mixtures of non-toxic chemicals: "solubility addition" increases exposure for solid hydrophobic chemicals. Environ Sci Technol 2013, 47, (4), 2026-33.

30. Lin, Z. F.; Du, J. W.; Yin, K. D.; Wang, L. S.; Yu, H. X., Mechanism of concentration addition toxicity: they are different for nonpolar narcotic chemicals, polar narcotic chemicals and reactive chemicals. Chemosphere 2004, 54, (11), 1691-1701.

31. Smith, E.; Wraige, E.; Donkin, P.; Rowland, S., Hydrocarbon humps in the marine environment: Synthesis, toxicity, and aqueous solubility of monoaromatic compounds. Environ Toxicol Chem 2001, 20, (11), 2428-2432. 
32. Echeveste, P.; Dachs, J.; Berrojalbiz, N.; Agusti, S., Decrease in the abundance and viability of oceanic phytoplankton due to trace levels of complex mixtures of organic pollutants. Chemosphere 2010, 81, (2), 161-168.

33. Greenberg, M. S.; Chapman, P. M.; Allan, I. J.; Anderson, K. A.; Apitz, S. E.; Beegan, C.; Bridges, T. S.; Brown, S. S.; Cargill, J. G. t.; McCulloch, M. C.; Menzie, C. A.; Shine, J. P.; Parkerton, T. F., Passive sampling methods for contaminated sediments: risk assessment and management. Integr Environ Assess Manag 2014, 10, (2), 224-36.

34. Muijs, B.; Jonker, M. T., Assessing the bioavailability of complex petroleum hydrocarbon mixtures in sediments. Environ Sci Technol 2011, 45, (8), 3554-61.

35. Rojo-Nieto, E.; Smith, K. E. C.; Perales, J. A.; Mayer, P., Recreating the seawater mixture composition of HOCs in toxicity tests with Artemia franciscana by passive dosing. Aquatic Toxicol 2012, 120, 27-34.

36. Arey, J. S.; Nelson, R. K.; Xu, L.; Reddy, C. M., Using comprehensive two-dimensional gas chromatography retention indices to estimate environmental partitioning properties for a complete set of diesel fuel hydrocarbons. Anal Chem 2005, 77, (22), 7172-82.

37. Nabi, D.; Gros, J.; Dimitriou-Christidis, P.; Arey, J. S., Mapping environmental partitioning properties of nonpolar complex mixtures by use of GC x GC. Environ Sci Technol 2014, 48, (12), 6814-26.

38. Antle, P. M.; Zeigler, C. D.; Livitz, D. G.; Robbat, A., Two-dimensional gas chromatography/mass spectrometry, physical property modeling and automated production of component maps to assess the weathering of pollutants. J Chromatogr A 2014, 1364, 223-33.

39. Lohmann, R., Critical review of low-density polyethylene's partitioning and diffusion coefficients for trace organic contaminants and implications for its use as a passive sampler. Environ Sci Technol 2012, 46, (2), 606-18.

40. $\quad$ Fernandez, L. A.; Lao, W.; Maruya, K. A.; White, C.; Burgess, R. M., Passive sampling to measure baseline dissolved persistent organic pollutant concentrations in the water column of the Palos Verdes Shelf Superfund site. Environ Sci Technol 2012, 46, (21), 1193747.

41. Lydy, M. J.; Landrum, P. F.; Oen, A. M.; Allinson, M.; Smedes, F.; Harwood, A. D.; Li, H.; Maruya, K. A.; Liu, J., Passive sampling methods for contaminated sediments: state of the science for organic contaminants. Integr Environ Assess Manag 2014, 10, (2), 167-78.

42. Fernandez, L. A.; Harvey, C. F.; Gschwend, P. M., Using performance reference compounds in polyethylene passive samplers to deduce sediment porewater concentrations for numerous target chemicals. Environ Sci Technol 2009, 43, (23), 8888-94. 43. Lampert, D. J.; Thomas, C.; Reible, D. D., Internal and external transport significance for predicting contaminant uptake rates in passive samplers. Chemosphere 2015, 119, 910916.

44. US Environmental Protection Agency Overview of United Heckathorn Co. Superfund Site. $\quad$ http://yosemite.epa.gov/r9/sfund/r9sfdocw.nsf/BySite/United Heckathorn Co.?OpenDocument (accessed April 2015),

45. Damage, Assessment, Remediation and Restoration Program, NOAA Island End River, MA Case Overview. http://www.darrp.noaa.gov/northeast/island/ (accessed April 2015), 


\title{
Chapter 2
}

\section{Estimating phospholipid membrane-water partition coefficients using comprehensive two-dimensional gas chromatography}

\author{
A. Patricia Tcaciuc ${ }^{1,2} *$, Robert K. Nelson ${ }^{2}$, Christopher M. Reddy ${ }^{2}$, and Philip M. Gschwend ${ }^{1}$ \\ ${ }^{1}$ Department of Civil and Environmental Engineering, Massachusetts Institute of Technology, \\ Cambridge, MA 02138, USA \\ ${ }^{2}$ Department of Marine Chemistry and Geochemistry, Woods Hole Oceanographic Institution, \\ Woods Hole, MA 02543, USA
}

Reproduced with permission from Environmental Science \& Technology, 46 (6), 3449-56 Copyright 2012 American Chemical Society

\section{Citation}

Tcaciuc, A. P.; Nelson, R. K.; Reddy, C. M.; Gschwend, P. M., Estimating phospholipid membrane-water partition coefficients using comprehensive two-dimensional gas chromatography. Environmental Science \& Technology 2012, 46, (6), 3449-56. 


\begin{abstract}
Recent studies have shown that phospholipid membrane-water partition coefficients of organic chemicals can be used to predict bioaccumulation and type I narcosis toxicity more accurately than the traditional $K_{O W^{-}}$-based approach. In this paper, we demonstrate how comprehensive twodimensional gas chromatography $(\mathrm{GC} \times \mathrm{GC})$ can be used to estimate such membrane-water partition coefficients $\left(K_{\mathrm{PLWS}}\right)$, focusing in particular on phosphatidyl choline based lipids. This method performed well for a set of 38 compounds, including polycyclic aromatic hydrocarbons, polychlorinated benzenes and biphenyls, and substituted benzenes including some phenols and anilines. The average difference between the estimated and the measured log $K_{\text {PLW }}$ values of $0.47 \log$ units is smaller than in the case of a $\log K_{\mathrm{OW}}$ correlation approach but larger than for a polyparameter model based approach in which $\log K_{\mathrm{PLW}}$ is described by a set of five solutesolvent interactions. However, the GC $\times$ GC based method has the advantage that it can be applied to mixtures of chemicals that are not completely identified, such as petroleum hydrocarbon mixtures. At the same time, our application of the $\mathrm{GC} \times \mathrm{GC}$ method suffered larger errors when applied to certain hydrogen bonding compounds due to the inability of the GC $\times \mathrm{GC}$ capillary columns phases that we used to participate in hydrogen bond donation.
\end{abstract}




\subsection{Introduction}

The bioaccumulation potential of organic contaminants is a key factor in environmental risk assessment of hydrophobic organic chemicals (HOCs). Compounds, for which the rate of biotransformation is slow compared to uptake, tend to accumulate in the lipids of exposed organisms, as dictated by their lipid-water or lipid-air partition coefficients ${ }^{1}$. One approach for calculating bioaccumulation relies on bioaccumulation/bioconcentration factors, obtained from linear free energy relationship correlations involving $K_{\mathrm{OW}}$, the $n$-octanol-water partition coefficient ${ }^{2}$. This approach assumes that the partition properties of all types of lipids are the same, although recent studies ${ }^{3,4}$ show that significant differences exist between the partitioning of chemicals into storage- versus membrane-lipids. In general, storage lipids consist of triacylglycerides (i.e., three aliphatic side-chains attached to a glycerol moiety). In contrast, membrane lipids are predominantly diacylglycerides (i.e., only two aliphatic side-chains attached to the glycerol unit), with a polar group attached at the third oxygen (Figure 2.1). This structural nature helps them form bilayers, a critical feature of biological membranes. For important environmental contaminants such as phenols Sandermann et al. ${ }^{3}$ found that partitioning into storage lipids can be as much as a factor of ten lower than the partitioning into membrane lipids, whereas for dichlorodiphenyltrichloroethane (DDT), partitioning into storage lipids was a factor of ten higher. This could be explained, at least in part, by the fact that unlike the triacylgliceride storage lipids, the diacylglicerides have moieties that can function as electron density acceptors. Thus, in order to accurately predict bioaccumulation, both the phospholipid-water and triglyceride-water partition coefficients must be known. This is of particular importance in smaller organisms, such as plankton, in which the proportion of membrane-to-storage lipids is $\operatorname{larger}^{3}$ (e.g. in plankton membrane lipids are $90 \%$ of total lipids, whereas in fish the membrane lipids account for $22 \%$ to $69 \%$ of the total lipids ${ }^{5}$ ). In addition, the correlation between the bioconcentration factor $(\mathrm{BCF})$ and $K_{\mathrm{OW}}$ has been shown to break down for certain classes of compounds, including highly hydrophobic HOCs with $\log K_{\mathrm{OW}}$ greater than $6^{6}$, and recent studies suggest membrane-water partition coefficients are better predictors for BCF than $K_{\mathrm{OW}}{ }^{7}$.

The differential affinities of contaminants for the two different lipid classes are also relevant from a toxicological perspective. Membranes have been identified as the target site for nonspecific (or type I) narcosis toxicity ${ }^{8}$. Therefore, by knowing the partition coefficient of a particular contaminant into membrane lipids, one can calculate its activity at the target site of 
toxicity and better evaluate the potential for toxic effects ${ }^{9}$. Generally, such baseline toxicity has been obtained, similarly to BCFs, via a correlation against $K_{\mathrm{OW}}$. However, Vaes et al. ${ }^{10}$ found that by using the membrane-water partition coefficient as a predictor instead of $K_{\mathrm{OW}}$, (1) the relationship can be extended to a larger set of compounds, and (2) lethal body burdens (LBBs) can be better predicted by considering the differential partitioning into two separate lipid compartments. In addition, evaluating the partitioning into storage lipids is also relevant because storage lipids are more labile than membrane lipids (i.e. the amount of storage lipids is known to vary with season), and because storage lipids are mobilized during reproduction, leading to transfer of contaminants to offspring during embryo development or lactation phase.

Experimentally, determination of phospholipid-water partition coefficients ( $\left.K_{\mathrm{PLW}}\right)$ has focused on phosphatidyl cholines (PCs) with various side chains, as PCs readily form vesicles in water and because PCs are one of the most common components of membrane lipids in higher organisms ${ }^{11}$. These coefficients have been measured for a wide range of organic compounds including known environmental contaminants such as polychlorinated biphenyls $(\mathrm{PCB})^{3,7,12-14}$, polycyclic aromatic hydrocarbons $(\mathrm{PAHs})^{7,15,16}$, chlorobenzenes $(\mathrm{CBs}),{ }^{7,12,15-18}$ and other compound classes. ${ }^{18}$ Unfortunately, for many compounds, there can be significant variability in the available data (e.g., $K_{\mathrm{PLW}}$ for PCB congener \#155 (2,2',4,4',6,6'-hexachlorobiphenyl) varies by more than two orders of magnitude between different measurements ${ }^{4,13}$ ). This may be due to experimental artifacts, but the data variation may also be caused by differences in the composition of aliphatic side chains or experimental temperatures that affect the phase of the lipids in question. At temperatures below the phase transition temperature (i.e., in the rigid gel phase), the aliphatic carbon atoms of the hydrophobic side chains reside primarily in the anti conformation; whereas above the phase transition temperature (i.e., in the liquid crystalline state), the gauche conformation becomes energetically favorable, leading to a more fluid, less well packed membrane. ${ }^{19}$ Thus, the ability of the liposome to accumulate contaminants is higher in the liquid crystalline phase than in the rigid gel phase. ${ }^{17}$ The value of the phase transition temperature is, in turn, affected by the choice of lipid side chain, with smaller chains and higher degrees of unsaturation leading to lower transition temperatures. 

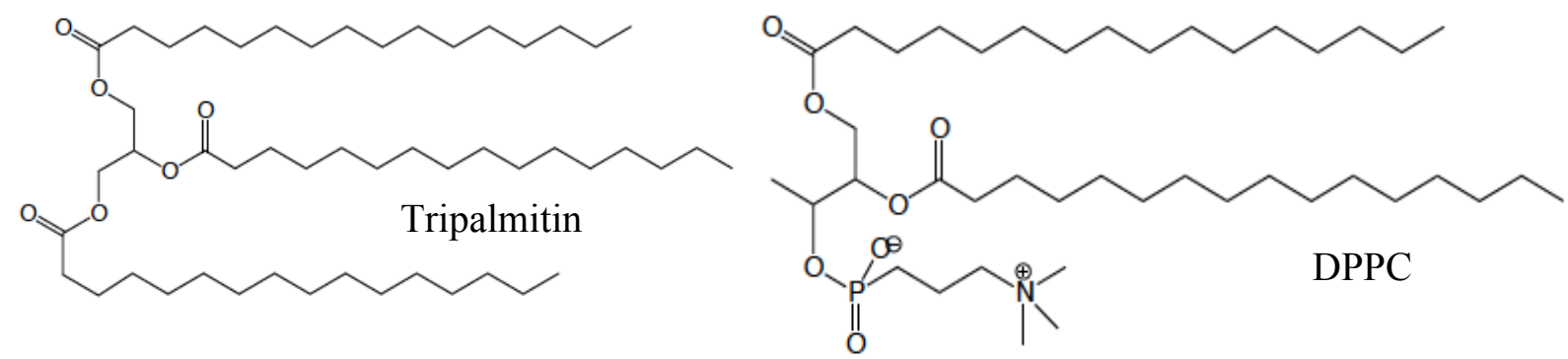

Figure 2.1 Examples of a triglyceride storage lipid, tripalmitin (C51H98O6, MW $807.34 \mathrm{~g} / \mathrm{mol}$ ), and a membrane phospholipid, dipalmitophosphatidyl choline (DPPC, C40H80NO8P, MW $734.04 \mathrm{~g} / \mathrm{mol})$

In this study, we present a new method of estimating the $K_{\text {PLW }}$ values of organic chemicals based on their retention behavior on a comprehensive two-dimensional gas chromatography system $(\mathrm{GC} \times \mathrm{GC})$. Current experimental determinations of $K_{\mathrm{PLW}}$ values require phospholipid vesicles to be formed in a reproducible fashion and incubation experiments to be performed in such a way to ensure liposome stability and enough time for equilibration of highly hydrophobic compounds. In addition, $K_{\mathrm{PLW}}$ determinations are subject to variability due to exact nature of lipid and experimental temperature, as mentioned above. The method of estimating $K_{\text {PLW }}$ values proposed here bypasses such methodological difficulties, and it can be applied when dealing with compounds for which experimental analysis is difficult.

Furthermore, the $\mathrm{GC} \times \mathrm{GC}$ method can be used to evaluate the potential for baseline (type I) narcosis toxicity of mixtures of chemicals, such as those in petroleum, which are not completely identified. Several studies have shown that components of the unresolved complex mixtures associated with petroleum contamination, can cause baseline narcosis type I toxic effects in invertebrates, but a method is still needed to evaluate this toxicity. The high separation power given by $\mathrm{GC} \times \mathrm{GC}$, coupled with the ability to give values of $K_{\mathrm{PLW}}$ for each of the mixture's components, which can in turn help us calculate the concentration of narcosis pollutants at their site of action, makes this technique ideal for estimating the cumulative baseline narcosis toxicity of such a mixture. The focus of this paper will be obtaining the values of $K_{\mathrm{PLW}}$ from a GC $\times \mathrm{GC}$ chromatogram, while the application to mixture toxicity will be addressed in a subsequent paper. 


\subsubsection{Background}

The advent of GC $\times$ GC has greatly improved our ability to separate and characterize components of complex organic mixtures; and recent studies show that with appropriate training sets, a range of physico-chemical properties can be estimated from the retention behaviors of the analytes including their vapor pressures and octanol-water partition coefficients. ${ }^{20}$ In $\mathrm{GC} \times \mathrm{GC}$, the effluent from the first column is trapped, focused, and injected onto a second, shorter column at discrete time intervals. The stationary phase of the first dimension column is typically nonpolar (e.g., polydimethylsiloxane), resolving compounds based chiefly on their London dispersive interactions with the stationary phase (interactions dependent on a compound's molecular volume and polarizability). In the second dimension column, due to the presence of the phenyl groups (stationary phase is 50\% phenyl polysilphenylene-siloxane, Figure S1), additional intermolecular interactions are also possible, notably hydrogen bond acceptance by the stationary phase (i.e. the contribution of a compound's polarizability, dipole and induce dipole and electron acceptor character to the overall retention increase by factors of 4,3 and 2, respectively, as will be discussed in section 2.4.1 and in Table 2.2). The apolar nature of the stationary phase mimics the partitioning from gas phase to a lipid phase, whereas the more polar nature of the second dimension reflects interactions that govern the partitioning from gas phase to water. Since $K_{\mathrm{PLW}}$ is a ratio of the lipid/gas and water/gas partition coefficients, then we expect that $\log K_{\mathrm{PLW}}$ will be positively correlated with the first dimension retention time, and negatively with respect to the second dimension. Mathematically this translates into a relationship of this form for the calculation of $\log K_{\mathrm{PLW}}$ :

$$
\log K_{\mathrm{PLW}}=\mathrm{a} * T_{1}+\mathrm{b} * T_{2}+\mathrm{c}
$$

where $T_{1}$ and $T_{2}$ are the retention times of compound of interest in the first and second dimension, respectively.

Determination of partition coefficients, such as $\log K_{\mathrm{OW}}$, has also been previously done using high pressure liquid chromatography and reverse phase column materials (HPLC). The advantage of this technique over GC is the presence of an aqueous phase, which directly captures the behavior of organic chemicals in water. However, in HPLC it takes a very long time to elute most compounds with only water; and if one uses an organic co-solvent, one has to train the system to correct for co-solvent effects. Also, compared to GC, LC is not as effective at separating complex mixtures, thereby limiting one's ability to examine such real world exposures 
to mixtures. Further, the detector response (e.g., absorbance or fluorescence) is not anywhere near as constant from analyte to analyte in HPLC as that seen with a flame ionization detector (FID); hence HPLC detectors do not allow as dependable a simultaneous quantification of mixture components as the FID when examining mixtures such as those in petroleum hydrocarbons. Such contaminant quantification, when combined with key physical chemical properties like $K_{\text {PLW }}$ values of each eluting peak, should allow eventual estimation of integrated membrane doses from mixtures (the long range goal of our work).

\subsection{Materials and Methods}

\subsubsection{Preparation of solutions.}

Most of the compounds used in the training sets were purchased as mixtures or individual compounds from Ultra Scientific, Inc. with the exception of the ones in the miscellaneous group, which were purchased as individual compounds from Sigma-Aldrich Co. Neat compounds were dissolved in dichloromethane and stock solutions were diluted to appropriate levels for GC $\times$ GC - FID analysis $(\sim 1-10 \mathrm{ng} / \mu \mathrm{L})$.

\subsubsection{Selection of training set compounds.}

We selected known environmental contaminants such as PCBs, PAHs, chlorobenzenes (CBs), as well as several structurally diverse benzene derivatives like phenols, anilines and nitroaromatics previously found to cause narcosis. ${ }^{\mathbf{1 0}}$ We chose the values of $K_{P L W}$ for which the experiments were performed at temperatures at which the liposomes were in the liquid crystalline phase, since biological membranes are mostly found in this state at environmental conditions (for example, transition phase temperature of egg phosphatidyl choline is $-10 \pm 5$ ${ }^{\circ} \mathrm{C}^{14}$ ). Additionally, in the liquid crystalline phase, the $K_{P L W}$ dependence with temperature is small, on the order of 0.1-0.2 log units per $10{ }^{\circ} \mathrm{C},{ }^{7,14,17}$ whereas a sharp change in partition behavior occurs below the transition phase temperature (e.g., for chlorobenzene there is a $1.6 \log$

units difference in $K_{P L W}$ between the two lipid states). ${ }^{17}$ After critically reviewing the available data (see Supplemental Information-1, Table S2) and checking it for consistency, we obtained the training set displayed in Table 2.1. The average $\log K_{P L W}$ was used when multiple $K_{P L W}$ values were available from different experimental setups. The regression of $\log K_{P L W}$ values 
against the two retention times (Eq. 1) was performed using the Data Analysis feature in Microsoft Excel 2004.

\subsubsection{GC $\times$ GC analysis.}

GC $\times$ GC analyses were performed on an Agilent 7890A gas chromatograph, equipped with a $7683 \mathrm{split} / \mathrm{splitless}$ injector, two capillary gas chromatography columns, a quad jet modulator (LECO Corporation, St Joseph, MI), and flame ionization detector (FID). The samples were injected in splitless mode. The inlet temperature was set at $300{ }^{\circ} \mathrm{C}$ and the purge valve was opened after $1 \mathrm{~min}$. The carrier gas used was $\mathrm{H}_{2}$, set at a flow rate of $1 \mathrm{~mL} / \mathrm{min}$ throughout the run. Using sequential pentane injections at 10 minute intervals, we determined that the breakthrough time through the second dimension column decreased by $\sim 30 \%$ throughout the run (from 1.710 seconds to 1.150 seconds), indicating that the flow rate in fact speeds up throughout the run. First dimension separations were performed using a 100\% dimethylpolysiloxane capillary column (Restek, RTX-1, $0.25 \mathrm{~mm}$ inner diameter, $0.24 \mu \mathrm{m}$ film thickness, $27.5 \mathrm{~m}$ length), which was ramped from $40{ }^{\circ} \mathrm{C}(0.5 \mathrm{~min}$ hold $)$, to $333{ }^{\circ} \mathrm{C}$ at $4.92{ }^{\circ} \mathrm{C} / \mathrm{min}$. Compounds exiting the first column were cryogenically trapped and re-injected (modulated) onto the second column at $6 \mathrm{~s}$ intervals, via the quad jet modulator. The cold jet was dry liquid $\mathrm{N}_{2}$. The hot jet was set at $40{ }^{\circ} \mathrm{C}$ above the temperature of the first dimension oven. The second column was a $50 \%$ phenyl polysilphenylene-siloxane capillary column (SGE BPX-50, $0.10 \mathrm{~mm}$ ID, $0.25 \mu \mathrm{m}$ thickness, $1.5 \mathrm{~m}$ length), and it was programmed from $55^{\circ} \mathrm{C}(0.5 \mathrm{~min}$ hold $)$ and ramped to 348 ${ }^{\circ} \mathrm{C}$ at $4.92{ }^{\circ} \mathrm{C} / \mathrm{min}$, which maintained a constant offset of $15{ }^{\circ} \mathrm{C}$ between the two columns throughout the run. The FID was set at $330{ }^{\circ} \mathrm{C}$ and sampled at $100 \mathrm{~Hz}$. This relatively fast temperature program sacrificed part of the separation power, but a wide range of compounds ( $n$ $\mathrm{C}_{8}$ to $n-\mathrm{C}_{34}$ alkanes in the first dimension) were eluted in a time efficient manner, suited to processing large sample sets as well as complex mixtures such as spilled petroleum. The variability of the $\mathrm{GC} \times \mathrm{GC}$ retention times from run to run was very small (variations of less than $0.01 \mathrm{~min}$ in the first dimension and of less than $0.03 \mathrm{~s}$ in the second dimension observed throughout all the runs). 
Table 2.1 Experimental and predicted $\log K_{\mathrm{PLW}}$ using three methods: GC $\times$ GC, polyparameter model $^{\mathrm{a}}$, and $\log K_{\mathrm{PLW}}=1.01 \log K_{\mathrm{OW}}+0.12^{\mathrm{a}}$

\begin{tabular}{|c|c|c|c|c|c|c|}
\hline \# & Compound & $\begin{array}{l}\text { Experimental } \\
\text { conditions }^{\mathrm{b}}\end{array}$ & $\begin{array}{l}\log \\
K_{\mathrm{PLW}} \\
\end{array}$ & $\begin{array}{l}\text { This } \\
\text { study }\end{array}$ & $\begin{array}{l}\text { Poly-param } \\
\text { model }\end{array}$ & $\begin{array}{l}\log \\
K_{\mathrm{OW}} \text { fit } \\
\end{array}$ \\
\hline \multicolumn{7}{|c|}{$\mathrm{PCBs}$} \\
\hline 1 & 2-chlorobiphenyl (\#1) & soy PC $\left(25^{\circ} C\right)^{d}$ & 4.83 & 4.68 & 4.47 & 4.97 \\
\hline 2 & 2,2',5,5'-tetrachlorobiphenyl (\#52) & $\begin{array}{l}\text { soy PC }\left(25^{\circ} \mathrm{C}\right)^{\mathrm{d}} \text {, } \\
\text { POPC }\left(20{ }^{\circ} \mathrm{C}\right)^{\mathrm{e}} \text {, } \\
\text { DMPC }\left(2{ }^{\circ} \mathrm{C}\right)^{\mathrm{e}} \text {, } \\
\text { DMPC }\left(25^{\circ} \mathrm{C}\right)^{\mathrm{f}}\end{array}$ & 6.12 & 6.71 & 5.84 & 6.02 \\
\hline 3 & 2,3,4,5- tetrachlorobiphenyl (\#61) & soy $\operatorname{PC}\left(25^{\circ} \mathrm{C}\right)^{\mathrm{d}}$ & 7.15 & 7.01 & 6.24 & 6.59 \\
\hline 4 & 2,2',4,4',6,6'-hexachlorobiphenyl (\#155) & soy $\mathrm{PC}\left(25^{\circ} \mathrm{C}\right)^{\mathrm{d}}$ & 7.65 & 7.47 & 6.44 & 7.48 \\
\hline 5 & 2,2',3,3',4,4'-hexachlorobiphenyl (\#128) & soy $\mathrm{PC}\left(25^{\circ} \mathrm{C}\right)^{\mathrm{d}}$ & 7.88 & 7.71 & 7.13 & 7.02 \\
\hline 6 & 2,2',3,3',6,6'-hexachlorobiphenyl (\#136) & $\operatorname{POPC}\left(25^{\circ} \mathrm{C}\right)^{\mathrm{a}}$ & 6.50 & 7.16 & 6.67 & 7.31 \\
\hline \multicolumn{7}{|c|}{ PAHs } \\
\hline 7 & naphthalene & $\operatorname{egg} \mathrm{PC}\left(20^{\circ} \mathrm{C}\right)^{\mathrm{g}}$ & 3.38 & 3.09 & 3.58 & 3.52 \\
\hline 8 & phenanthrene & $\begin{array}{l}\text { POPC }\left(20^{\circ} \mathrm{C}\right)^{\mathrm{e}, \mathrm{h}}, \\
\operatorname{egg} \operatorname{PC}\left(20^{\circ} \mathrm{C}\right)^{\mathrm{g}}\end{array}$ & 4.91 & 4.99 & 4.91 & 4.74 \\
\hline 9 & anthracene & $\begin{array}{l}\operatorname{POPC}\left(20^{\circ} \mathrm{C}\right)^{\mathrm{e}, \mathrm{h}}, \\
\operatorname{egg} \operatorname{PC}\left(20^{\circ} \mathrm{C}\right)^{\mathrm{g}}\end{array}$ & 5.04 & 5.08 & 5.00 & 4.71 \\
\hline 10 & fluoranthene & $\operatorname{POPC}\left(20^{\circ} \mathrm{C}\right)^{\mathrm{e}, \mathrm{h}}$ & 5.68 & 5.81 & 5.51 & 5.39 \\
\hline 11 & pyrene & $\begin{array}{l}\text { POPC }\left(20^{\circ} \mathrm{C}\right)^{\mathrm{e}, \mathrm{h}}, \\
\operatorname{egg} \operatorname{PC}\left(20^{\circ} \mathrm{C}\right)^{\mathrm{g}}\end{array}$ & 5.48 & 5.57 & 5.60 & 5.35 \\
\hline 12 & benz $[a]$ anthracene & $\operatorname{POPC}\left(20^{\circ} \mathrm{C}\right)^{\mathrm{e}, \mathrm{h}}$ & 6.53 & 6.64 & 6.37 & 6.04 \\
\hline 13 & chrysene & $\operatorname{POPC}\left(20^{\circ} \mathrm{C}\right)^{\mathrm{e}, \mathrm{h}}$ & 6.49 & 6.44 & 6.38 & 6.09 \\
\hline 14 & benzo $[b]$ fluoranthene & $\operatorname{POPC}\left(20^{\circ} \mathrm{C}\right)^{\mathrm{e}, \mathrm{h}}$ & 7.23 & 7.25 & 6.62 & 6.22 \\
\hline 15 & benzo $[k]$ fluoranthene & $\operatorname{POPC}\left(20^{\circ} \mathrm{C}\right)^{\mathrm{e}, \mathrm{h}}$ & 7.24 & 7.25 & 6.82 & 6.22 \\
\hline 16 & benzo $[a]$ pyrene & $\operatorname{POPC}\left(20^{\circ} \mathrm{C}\right)^{\mathrm{e}, \mathrm{h}}$ & 7.37 & 6.85 & 7.00 & 6.08 \\
\hline 17 & dibenz $[a, h]$ anthracene & $\operatorname{POPC}\left(20^{\circ} \mathrm{C}\right)^{\mathrm{e}, \mathrm{h}}$ & 7.80 & 7.64 & 7.78 & 6.69 \\
\hline 18 & indeno $[1,2,3-c d]$ pyrene & $\operatorname{POPC}\left(20^{\circ} \mathrm{C}\right)^{\mathrm{e}, \mathrm{h}}$ & 7.97 & 7.62 & 7.25 & 6.69 \\
\hline 19 & benzo $[g, h, i]$ perylene & $\operatorname{POPC}\left(20^{\circ} \mathrm{C}\right)^{\mathrm{e}, \mathrm{h}}$ & 7.91 & 7.26 & 7.55 & 6.94 \\
\hline \multicolumn{7}{|c|}{$\mathrm{CBs}$} \\
\hline 20 & chlorobenzene & $\begin{array}{l}\text { DMPC }\left(36{ }^{\circ} \mathrm{C}\right)^{\mathrm{i}} \text {, } \\
\text { DMPC }\left(26.5^{\circ} \mathrm{C}\right)^{\mathrm{f}}\end{array}$ & 2.91 & 2.39 & 2.92 & 3.05 \\
\hline 21 & 1,3-dichlorobenzene & $\operatorname{DMPC}\left(26.5^{\circ} \mathrm{C}\right)^{\mathrm{g}}$ & 3.71 & 2.81 & 3.58 & 3.69 \\
\hline 22 & 1,4-dichlorobenzene & $\operatorname{DMPC}\left(26.5^{\circ} \mathrm{C}\right)^{\mathrm{g}}$ & 3.57 & 2.79 & 3.54 & 3.59 \\
\hline 23 & 1,2-dichlorobenzene & $\begin{array}{l}\text { DMPC }\left(26.5^{\circ} \mathrm{C}\right)^{\mathrm{g}} \text {, } \\
\operatorname{egg} \text { PC }\left(20^{\circ} \mathrm{C}\right)^{\mathrm{g}}\end{array}$ & 3.49 & 2.78 & 3.48 & 3.58 \\
\hline 24 & 1,2,4-trichlorobenzene & $\operatorname{DMPC}\left(26.5^{\circ} \mathrm{C}\right)^{\mathrm{g}}$ & 4.20 & 3.52 & 4.12 & 4.21 \\
\hline 25 & 1,2,3-trichlorobenzene & $\begin{array}{l}\text { POPC }\left(20{ }^{\circ} \mathrm{C}\right)^{\mathrm{e}}, \\
\text { DMPC }\left(36^{\circ} \mathrm{C}\right)^{\mathrm{i}}, \\
\text { egg PC }\left(20^{\circ} \mathrm{C}\right)^{\mathrm{g}}\end{array}$ & 4.08 & 3.53 & 4.12 & 4.30 \\
\hline 26 & 1,2,4,5-tetrachlorobenzene & $\operatorname{DMPC}\left(26.5^{\circ} \mathrm{C}\right)^{\mathrm{g}}$ & 4.49 & 4.43 & 5.05 & 4.77 \\
\hline 27 & pentachlorobenzene & DMPC $\left(26.5^{\circ} \mathrm{C}\right)^{\mathrm{g}}$ & 5.06 & 5.22 & 5.17 & 5.35 \\
\hline 28 & hexachlorobenzene & DMPC $\left(26.5^{\circ} \mathrm{C}\right)^{\mathrm{g}}$ & 5.56 & 6.08 & 5.64 & 5.98 \\
\hline \multicolumn{7}{|c|}{ Miscellaneous } \\
\hline 29 & $p$-xylene & $\operatorname{DMPC}\left(36^{\circ} \mathrm{C}\right)^{\mathrm{i}}$ & 2.98 & 2.71 & 3.12 & 3.30 \\
\hline 30 & aniline & $\operatorname{DMPC}\left(36^{\circ} \mathrm{C}\right)^{\mathrm{i}}$ & 1.63 & 1.96 & 1.59 & 1.03 \\
\hline
\end{tabular}




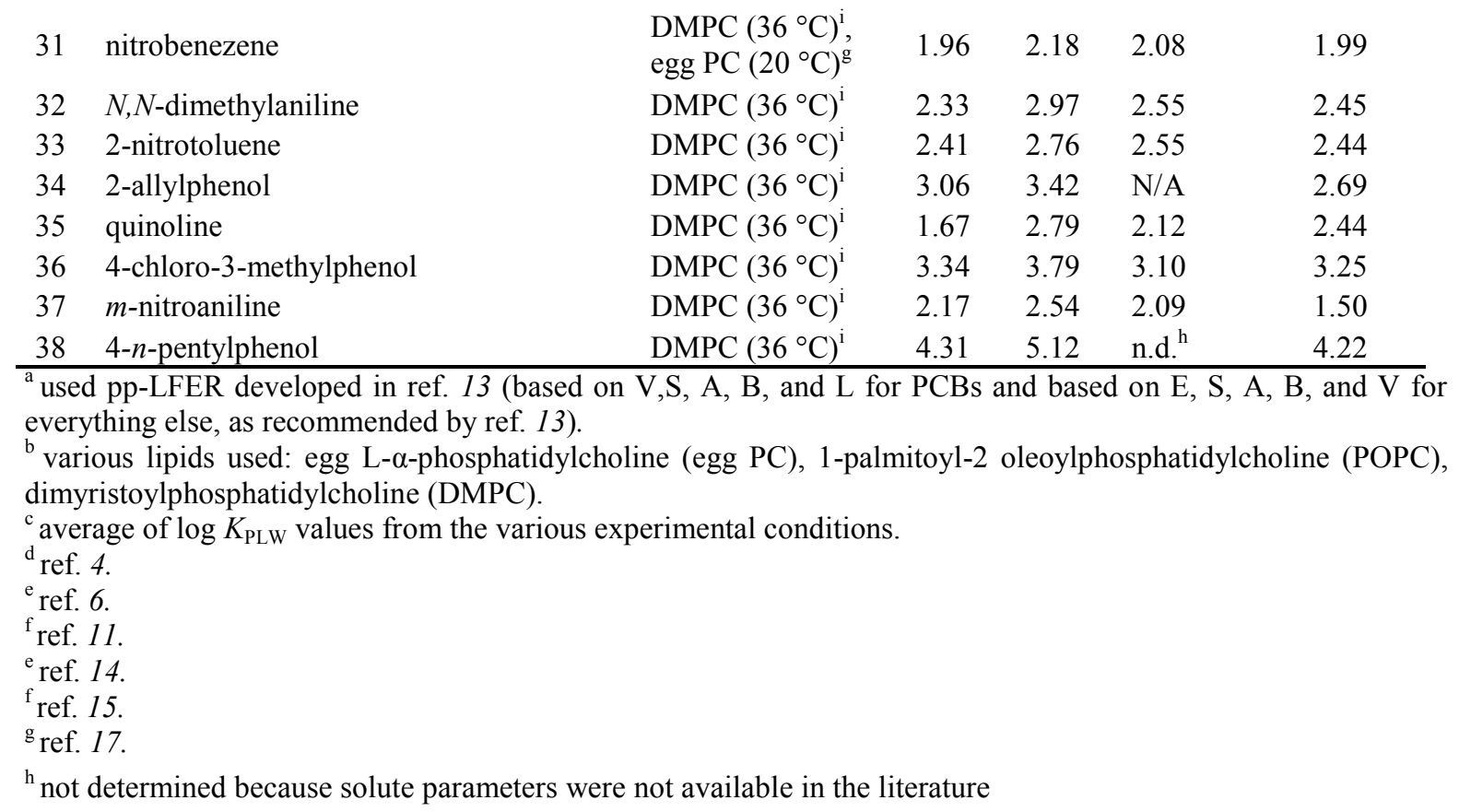

\subsection{Results}

\subsubsection{GC $\times$ GC system check and use of retention times to estimate log Kow values}

In order to test the validity of the $\mathrm{GC} \times \mathrm{GC}$ setup used, we first performed a regression of $\log$ $K_{\mathrm{OW}}$ against the two retention times. Based on the work done by Arey et al. ${ }^{20}, \mathrm{GC} \times \mathrm{GC}$ retention indices allow one to estimate $\log K_{\mathrm{OW}}$ with a standard error of about $0.2 \log$ units, using the same two stationary phases as the ones used in this study. However, we wanted to observe the consequences of using retention times instead of retention indices used by Arey et al. ${ }^{20} \mathrm{We}$ found that for a comparable training set (Figure S2, Table S1), consisting of only apolar and monopolar compounds (PAHs, PCBs and CBs), there was a good correlation between $\log K_{\mathrm{Ow}}$ and retention times:

$$
\begin{aligned}
& \log K_{\mathrm{OW}}=(0.165 \pm 0.006)^{*} T_{1}+(-1.33 \pm 0.09) * T_{2}+(3.32 \pm 0.16) \\
& \mathrm{N}=41, \mathrm{R}^{2}=0.957, \text { and } \mathrm{SE}=0.29
\end{aligned}
$$

As shown by Arey et al. ${ }^{19}$, retention indices can be reproduced by using two different instrument setups: different column lengths, temperature ramps and gas flows, but that would not be the case for retention times. Thus, we can obtain comparable results by using retention times 
instead of indices, with the downside that we would have to retrain the relationship if the instrument or the setup (e.g., column lengths or carrier flows) is changed. However, the task of retraining only involves running a set of standard mixtures and performing the two dimensional regression. In contrast, the calculation of retention indices of Arey et al. ${ }^{20}$ is significantly more involved mathematically, and requires GC $\times$ GC-specific parameters as inputs, such as hold up times or phase ratios of the columns.

\subsubsection{GC $\times$ GC-based estimation of $K_{P L W}$ values}

The training set used to calibrate the relationship between $K_{\mathrm{PLW}}$ and retention times included six PCBs, 13 PAHs, nine CBs and 10 benzene derivatives including phenols, nitroaromatic compounds, and anilines (Table 2.1). The regression using the reported $K_{\text {PLW }}$ values of this training set and the $\mathrm{GC} \times \mathrm{GC}$ retention times was quite good:

$$
\begin{aligned}
& \log K_{\mathrm{PLW}}=(0.208 \pm 0.010) * T_{1}+(-1.42 \pm 0.16) * T_{2}+(2.50 \pm 0.22) \\
& N=38, R^{2}=0.953, S E=0.45
\end{aligned}
$$

The statistics of this regression are clearly better (smaller standard error, and larger $R^{2}$ ) than if we use only the first dimension retention times. This can be seen by using the first retention times ( $T_{1}$ values) of our training set of compounds to find a fit as if we had used a one dimensional GC equipped with the same capillary column as the first dimension of the GC $\times$ GC, and operated in similar flow and temperature program conditions:

$$
\begin{aligned}
& \log K_{\mathrm{PLW}}=(0.127 \pm 0.009) * T_{1}+(1.22 \pm 0.30) \\
& N=38, R^{2}=0.843, S E=0.80
\end{aligned}
$$

To evaluate the effectiveness of the GC $\times$ GC-deduced correlation (Eq 3), we re-trained the relationship (Eq. 1) with 37 compounds and predicted the $\log K_{\mathrm{PLW}}$ of the $38^{\text {th }}$ compound, and we repeated this procedure for each compound in our training set. The predicted $\log K_{\text {PLW }}$ values (Table 2.1, Figure 2.2A) had an average deviation from the measured value of 0.47 or a factor of 3 in the $K_{\text {PLW }}$ (calculated as the square root of the sum of square deviations divided by number of observations minus 1). We note that four of the divergent compounds were chlorinated benzenes which were all estimated too low, while three were hydrogen bonding compounds which were all overestimated ( $N, N$-dimethylaniline, quinoline, $n$-pentylphenol). Suspecting that this bias may arise by inclusion of the polar compounds, we refit Eq. 1 excluding these compounds and found:

$$
\begin{aligned}
& \log K_{\mathrm{PLW}}=(0.170 \pm 0.010) * T_{1}+(-0.984 \pm 0.146) * T_{2}+(2.71 \pm 0.16) \\
& N=29, R^{2}=0.969, S E=0.30
\end{aligned}
$$


The expression greatly reduced the chlorobenzene's deviations from measured values (error now near 0.2 log unit) at the cost of no longer accurately estimating the polar compounds (error now near $1 \log$ unit).

\subsection{Discussion}

\subsubsection{Comparison of GC $\times$ GC method versus ppLFER and log Kow approaches}

Two other approaches have commonly been used to estimate $K_{\mathrm{PLW}}$ values. The first involves a correlation with octanol-water partitioning coefficients and the second entails use of a polyparameter linear free energy relationship (ppLFER). To ascertain the accuracy of our new GC $\times$ GC approach, we contrasted estimates made in this way with those derived from these other methods. In each case, the $K_{\mathrm{PLW}}$ estimation method was applied, when possible to all the compounds in our GC $\times$ GC training set, and an average deviation between the estimated and the measured value of $K_{\mathrm{PLW}}$ was calculated as the square root of the sum of squared deviations divided by number of observations minus 1 .

First, we compared the performance of the $\mathrm{GC} \times \mathrm{GC}$-based method of estimating $K_{\mathrm{PLW}}$ to a linear free energy relationship (LFER) approach based on $\log K_{\text {Ow. While many such }}$ relationships are available in the literature, we chose to use the one of Endo et al. ${ }^{14}$ because it was developed using the largest number of compounds $\left(\log K_{\mathrm{PLW}}=1.01 \log K_{\mathrm{OW}}+0.12 ; N=156\right.$, $S E=0.426, R^{2}=0.948$, Table 2.1). This method of estimating $K_{\mathrm{PLW}}$ showed larger deviations than the $\mathrm{GC} \times \mathrm{GC}$ method (average deviation between estimated and measured log $K_{\mathrm{PLW}}$ of 0.58 vs 0.47). Also the approach using $\log K_{\mathrm{OW}}$ increasingly underestimated $\log K_{\mathrm{PLW}}$ values for the highly hydrophobic PAHs, while the GC $\times$ GC method did not do so (Figure 2.2A vs. 2.2B). In addition, the $K_{\mathrm{OW}}$-based method depends on the availability of accurate $K_{\mathrm{OW}}$ values, which for PCBs for example can vary in the literature by more than one order of magnitude. ${ }^{21}$ 

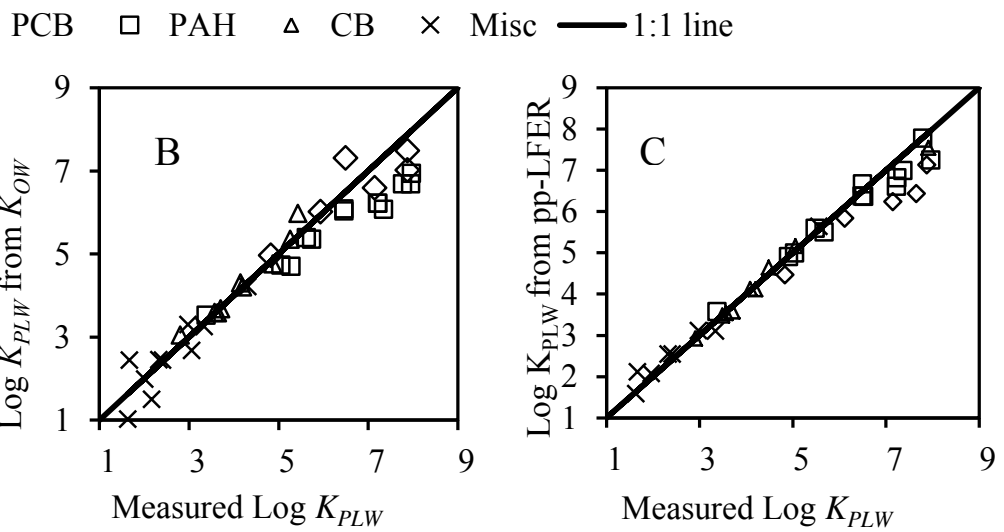

Figure 2.2 Comparison of $\log K_{\mathrm{PLW}}$ predicted from $\mathrm{GC} \times \mathrm{GC}$ (panel A), using $\log$ $K_{\mathrm{PLW}}=1.01 * \log K_{\mathrm{OW}}+0.12$ (ref. 13, Panel B), and polyparameter model ${ }^{13}$ (panel C) for different compounds (PCBs, PAHs, CBs and miscellaneous).

We also compared the $\mathrm{GC} \times \mathrm{GC}$-based method with results obtained using a polyparameter solvation model. In the polyparameter solvation model, ${ }^{22}$ the partitioning between two media $(\log K)$, such as water and phospholipids, can be described in terms of five dimensions of solutesolvent interactions using a relationship of the form:

$$
\log K=e^{*} E+s^{*} S+a^{*} A+b^{*} B+v^{*} V+c
$$

The capital letters refer to the solute parameters: $E$ (excess molar refraction and hence polarizability), $S$ (polarity), $A$ (hydrogen bond acidity), $B$ (hydrogen bond basicity), $V$ (solute size) and the small letters reflect the differential interactions of the solutes in the two partitioning phases. Previous investigations ${ }^{14}$ found that the best-fit interaction coefficients for phospholipid/water, olive oil/water and octanol/water partitioning systems have similar signs and magnitudes implying that the same intermolecular interactions govern partitioning in these systems (Table 2.2).

We applied the polyparameter equation developed for $\log K_{\text {PLW }}$ ppLFER by Endo et al. ${ }^{14}$ $\left(N=131, S E=0.277, R^{2}=0.979\right.$, Table 2, Eq. c) to all the compounds in our training set (Table 2.1, Figure 2.2C) with the exception of 4-n-pentylphenol and 2-allylphenol for which solute descriptors were not found in the literature. When we do this, for these 36 compounds, we find an average deviation between the estimated and the predicted $\log K_{\text {PLW }}$ of 0.38 . This is lower than the average deviation of 0.46 obtained when the $\mathrm{GC} \times \mathrm{GC}$-based method is used. The polyparameter model is especially able to better characterize the phenols, anilines, and 
nitroaromatic compounds (average deviation across the miscellaneous group of compounds of 0.23 compared to 0.59 in $\mathrm{GC} \times \mathrm{GC}$ approach). This is understandable because the polyparameter model takes into account a wider range of intermolecular interactions, such as the ability of compounds to donate electrons/accept hydrogens, which the stationary phases used in the GC $\times$ GC setup do not capture (Table 2.2). For example, Breitbach et al. ${ }^{23}$ investigated a series of phosphonium-based cations and found that when coupled with a $\mathrm{BF}_{4}{ }^{-}$or $\mathrm{Cl}^{-}$anions, the ionic liquids did show a small but significant $b$ character $(b=-0.5 \pm 0.1)$. Recently some ionic liquid columns have become commercially available (e.g., phosphonium cation coupled with a (trifluoromethylsulfonyl)imide anion, SLB®-IL59, Sigma Aldrich LLC, St Louis, MO), but their temperature limits are lower than those of BPX-50 (e.g., $300{ }^{\circ} \mathrm{C}$ for SLBß-IL59 but $330{ }^{\circ} \mathrm{C}$ isothermal and $350{ }^{\circ} \mathrm{C}$ programmed for BPX-50).

This limitation is apparent when one applies the ppLFER approach to explain partitioning behavior in GC systems. For the stationary phases we used, best fit ppLFER coefficients have been determined ${ }^{24}$ (Table 2.2), and these show similar $l$ coefficient values reflecting similar London interactions for both stationary phases, but increased $e, s$, and especially $a$ coefficients for the $50 \%$ phenyl phase of the second dimension column. However, what is most noteworthy is that for both of our stationary phases, $b$ is zero (neither of the two stationary phases can donate hydrogens) and this is the case for all commercially available stationary phases at this time. But for phospholipid-water partitioning, $b$ is nonzero and negative, as this term reflects the differential ability of water and phospholipids to donate protons/accept electrons to/from the compounds of interest.

However, both the polyparameter model as well as the $\mathrm{GC} \times \mathrm{GC}$ based method should be able to characterize equally well hydrophobic compounds like PCBs and PAHs. When applied to the PCBs in our training set, the polyparameter equation (c) in Table 2.2 estimates $\log K_{\mathrm{PLW}}$ with an average deviation of $0.79 \log$ units. In comparison, the average deviation obtained for the $K_{\mathrm{PLW}}$ of PCBs via the $\mathrm{GC} \times \mathrm{GC}$ method was 0.42 . One possible reason for this discrepancy may be the differences in the training sets. Interestingly, for the PCBs used in our training set and measured by Jabusch et al., ${ }^{4}$ there is an average difference of $0.8 \log$ units between the experimental value of $\log K_{\mathrm{PLW}}$ and the polyparameter model prediction, with the experimental value always being higher. In comparison, Endo et al. ${ }^{14}$ used a different PCB data set in developing their polyparameter model and thus, the differences between the two estimation 
methods appear to stem from the large variability in the available data on $K_{\mathrm{PLW}}$ values of PCBs. As with other partition constants, such as $K_{\mathrm{OW}}$, the $K_{\mathrm{PLW}}$ values for highly hydrophobic compounds like PCBs are difficult to observe due to low solubilities and long equilibration times.

Table 2.2 Poly-parameter model coefficients for retention behavior on two stationary phases (ref. 22, pg 100) similar to the ones used in the GC $\times$ GC setup of this work, as well as for calculating $\log K_{O W}, \log K_{P L W}$ and $\log K_{\text {olive oil/water }}$ (all from ref. 13).

\begin{tabular}{llllllll}
$\begin{array}{l}\text { Stationary phase or } \\
\text { partition coefficient }\end{array}$ & $c$ & $e$ & $s$ & $a$ & $b$ & $l$ & $v$ \\
\hline polydimethylsiloxane, $121{ }^{\circ} \mathrm{C}^{\mathrm{a}}$ & -0.19 & 0.024 & 0.190 & 0.125 & 0 & 0.498 & \\
polymethylphenylsiloxane, $121^{\circ} \mathrm{C}^{\mathrm{a}}$ & -0.372 & 0.071 & 0.653 & 0.263 & 0 & 0.518 & \\
$\log K_{\mathrm{PLW}}, 25{ }^{\circ} \mathrm{C}^{\mathrm{a}, \mathrm{b}}$ & 1.46 & -0.80 & -1.14 & -1.09 & -4.22 & 1.64 & \\
$\log K_{\mathrm{PLW}}, 25{ }^{\circ} \mathrm{C}^{\mathrm{c}}$ & 0.23 & 0.84 & -0.75 & 0.28 & -3.86 & & 3.37 \\
$\log K_{\mathrm{OW}}, 25{ }^{\circ} \mathrm{C}^{\mathrm{c}}$ & 0.09 & 0.56 & -1.05 & 0.03 & -3.34 & & 3.81 \\
$\log K_{\text {Olive oil } / \mathrm{W}}, 37^{\circ} \mathrm{C}^{\mathrm{c}}$ & 0.02 & 0.56 & -0.98 & -1.94 & -4.46 & & 4.22 \\
\hline
\end{tabular}

${ }^{a} \log \mathrm{K}=\mathrm{c}+\mathrm{e}^{*} \mathrm{E}+\mathrm{s} * \mathrm{~S}+\mathrm{a} * \mathrm{~A}+\mathrm{b} * \mathrm{~B}+1 * \mathrm{~L}$, the capital letters refer to descriptors of the compounds, previously explained in the text, with the exception of $\mathrm{L}$ which is the log of solute gas-liquid distribution constant on hexadecane at 298 $\mathrm{K}$ (also known as Ostwald solubility coefficient)

${ }^{b}$ adapted from ref. 13 to be in the same set of parameters as the pp-LFERs of the two stationary phases.

${ }^{c} \log \mathrm{K}=\mathrm{c}+\mathrm{e} * \mathrm{E}+\mathrm{s} * \mathrm{~S}+\mathrm{a} * \mathrm{~A}+\mathrm{b} * \mathrm{~B}+\mathrm{v} * \mathrm{~V}$.

\subsubsection{Limitations of the GC $\times$ GC-based method}

With our current choice of stationary phases in the $\mathrm{GC} \times \mathrm{GC}$, we expected that we would not be able to characterize compounds with a strong electron donating ( $\mathrm{H}^{+}$accepting) character as reflected in the polyparameter $B$ term. Neither of the two stationary phases can donate hydrogens (Table 2.2); yet, the correlation is able to predict compounds like PAHs (error 0.28), and PCBs (error 0.42) with non-zero $B$ character, as well as some of the miscellaneous compounds (error 0.59), such as aromatic amines. One explanation could be that the contribution of the $B$ character to the partitioning into phospholipids is minor compared to the contributions of the other interactions. This applies to PCBs for which $B$ ranges from 0.02 to $0.20^{25}$ rendering a maximum contribution of $0.8 \log$ units to the value of $\log K_{\text {PLW. The second }}$ possible explanation is that the $B$ character correlates with another descriptor, such as $E$ or $S$, which is true for PAHs (Figure S3). Arey at el. ${ }^{20}$ reached a similar conclusion when trying to investigate which kind of information can be provided from the retention behavior of diesel hydrocarbons on the same stationary phases, as used in this study. Lastly, the compounds that 
exhibit large $B$ values that do not correlate with either of the other descriptors, should reflect large errors in $\log K_{\mathrm{PLW}}$. This is true for some of the miscellaneous compounds such as quinoline (Figure S3). In order to reduce the errors associated with our method, we would need to employ better stationary phases, which could capture compounds with hydrogen-accepting character, but such GC phases are not currently available.

\subsubsection{Applications of the GC $\times$ GC-based method: estimating $\mathrm{K}_{\mathrm{PLW}}$ values for new compounds}

We applied this method to the prediction of $K_{\mathrm{PLW}}$ for a set of PCBs and organochlorine pesticides (OCPs) for which, to our knowledge, there are no available experimental data on $K_{\mathrm{PLW}}$. We compared our estimates against the polyparameter model of Endo et al. ${ }^{14}$ (Figure 2.3 and Table S3). For PCBs, the two methods agree well up to $\log K_{\mathrm{PLW}}$ of around 6 . Beyond that, the $\mathrm{GC} \times \mathrm{GC}$ method predicts consistently larger values than the polyparameter method, leading to an overall positive bias. This could be explained by the inability of the stationary phases to capture the hydrogen bond donation interaction, which has a negative contribution to $\log K_{\mathrm{PLW}}$ or by the difference in training sets mentioned earlier. A similar trend is observed for the organochlorine pesticides (Table S3), with the exception of a group of OCPs (heptachlor epoxide, methoxychlor, dieldrin, eldrin and endosulfan) which all contain one or more oxygen atoms. For these, the differences between the two predictive methods are on average $2.6 \log$ units, most likely due to their pronounced hydrogen-bond accepting character $(B$ terms larger than of all the compounds in the GC $\times$ GC training set).

Based on the results presented here, and the discussion on the limitation of the $\mathrm{GC} \times \mathrm{GC}$ method, we believe that this method can be accurately applied to compound classes such as petroleum hydrocarbons, PAHs, PCBs and CBs, that is, hydrophobic chemicals commonly assessed for their likely impacts via type I narcosis toxicity. Larger errors are expected when this method is applied to compounds which can accept hydrogen bonds (based on our training set, compounds with $B$ values greater than 0.4 tend to have deviations between the estimated and the measured $\log K_{\text {PLW }}$ greater than $0.5 \log$ units). However, we note that the method may still work for $B$ values greater than 0.4 , if the $\mathrm{B}$ character is correlated with another descriptor (for example PAHs have $B$ values greater than 0.4 , but there is a correlation within the PAH family between the $E$ and $B$ descriptors, as shown also in Figure S3). 


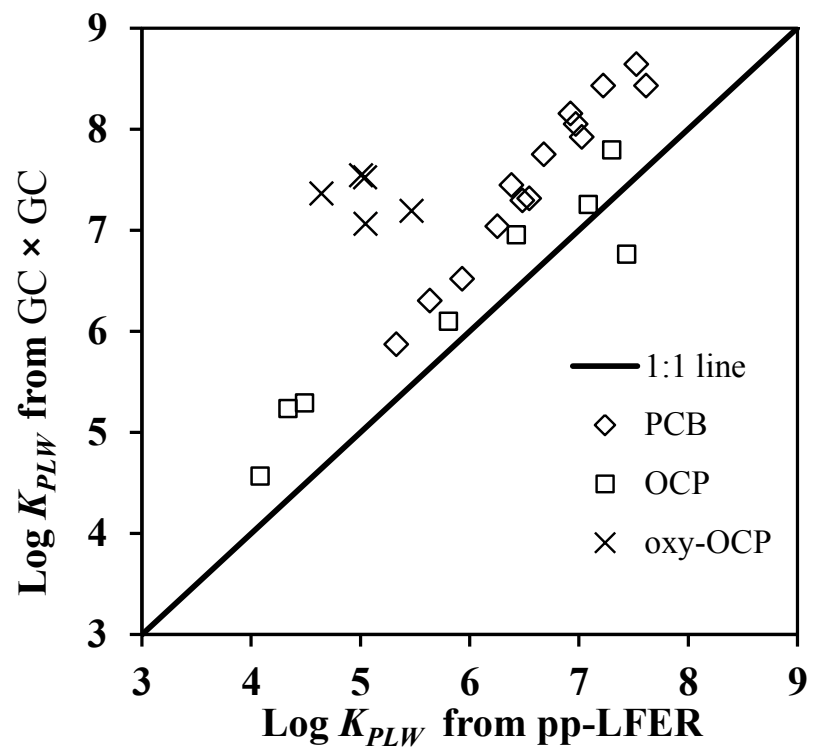

Figure 2.3 Comparison of $K_{\mathrm{PLW}}$ predicted from $\mathrm{GC} \times \mathrm{GC}$ and polyparameter model $^{13}$ for various PCBs and OCPs.

\subsubsection{Applications of the GC $\times$ GC-based method: estimating baseline narcosis risks}

The GC $\times$ GC method of estimating $K_{\mathrm{PLW}}$ values can also be applied to calculations of baseline (type I) narcosis toxicity of mixtures, e.g. petroleum hydrocarbon mixtures. For calculating baseline (type I) narcosis toxicity, we rely on two assumptions. First, we assume that all the components of the mixture partition to a certain extent in the membrane, contributing in an additive fashion to a type I narcosis effect. ${ }^{26}$ Secondly, we assume that all the analytes quantified in the $\mathrm{GC} \times \mathrm{GC}$ run have virtually the same flame ionization detector (FID) response factor. Consequently, one could start with the GC $\times$ GC chromatogram of a passive sampler extract in which the concentration of each peak/compound can be calculated using the relatively constant response factor of the FID. In addition, at each point in the $\mathrm{GC} \times \mathrm{GC}$ space, one can calculate the value of the $K_{\mathrm{PLW}}$ and the passive sampler-water partition coefficient by using equations such as Eq. 1. The integrated dose of contaminants inside the membrane lipid then becomes a sum across the entire GC $\times \mathrm{GC}$ space of all the calculated lipid concentrations of individual compounds. As opposed to other narcosis lipid models, such as the one proposed by McGrath et al., ${ }^{27}$ this approach would have the advantage that it does not require the identification of each single compound, nor specific knowledge about their effect concentration (i.e. the concentration required to produce a narcosis effect in $50 \%$ of the test organisms). 


\subsubsection{Applications of the GC $\times$ GC-based method: estimating bioaccumulation of mixtures}

For calculations of bioaccumulation, one would additionally require information about the proportion of storage versus membrane lipids, and the value of the partition constant between the triglycerides and water $\left(K_{\mathrm{TGW}}\right)$ at each point in the chromatogram. The values of $K_{\mathrm{TGW}}$ could be calculated with a relationship of the form of Eq 1, after running an appropriate training set of compounds with known $K_{\mathrm{TGW}}$ values on the $\mathrm{GC} \times \mathrm{GC}$, and finding the corresponding ppLFER regression coefficients. Then, assuming equilibrium with the environment, one could calculate the concentration of pollutants in each lipid compartment. One limitation of calculating bioaccumulation in this approach is that it would not apply to substances that are biotransformed at rates comparable to, or faster than, biouptake equilibration.

In this present study, we have shown that $\mathrm{GC} \times \mathrm{GC}$ retention behavior can be used to predict $K_{\text {PLW }}$ for a series of chemicals within about a factor of 3 . The results of the $\mathrm{GC} \times \mathrm{GC}$-based method compared well with those from two other $K_{\text {PLW }}$ prediction methods: a polyparameter model and a $\log K_{\mathrm{OW}}$ based LFER. The practical advantages of predicting $K_{\mathrm{PLW}}$ from GC $\times \mathrm{GC}$ retention behavior are (1) that it can be used to estimate $K_{\mathrm{PLW}}$ for compounds where experimental manipulations and analysis might be difficult, (for example hydrophobic PCBs with long equilibration timescales, like those in our training set and Figure 2.3) and (2) that it could also be applied to mixtures of hydrophobic chemicals that are likely to cause baseline narcosis toxicity (e.g. petroleum hydrocarbon mixtures), and for which separation, and characterization of individual components might not be feasible. Even though the relationship presented here between $\log K_{\mathrm{PLW}}$ and retention behavior is valid only for the particular GC $\times$ GC setup used in this study, the method is easily transferable to other GC $\times$ GC systems, as it simply involves (a) running an HOC training set on the system in use at the site, and (b) performing a simple regression on reported $K_{\mathrm{PLW}}$ values and the $\mathrm{GC} \times \mathrm{GC}$ retention times.

\section{Acknowledgments}

This publication is the result of research sponsored by The MIT Sea Grant College Program, under NOAA grant number NA06OAR4170019, project number 2008-R/RC-109. We also acknowledge funding from CICEET, the Cooperative Institute for Coastal and Estuarine Environmental Technology (award number 015553-001). The authors also acknowledge R. 
Scott Carr and Marion Nipper for their help with sample collection and useful discussions, and David Griffith for comments on the manuscript. 


\section{References}

1. Chiou, C. T., Partition coefficients of organic-compounds in lipid-water systems and correlations with fish bioconcentration factors. Environ Sci Technol 1985, 19, (1), 57-62.

2. US Environmental Protection Agency Methodology for Deriving Ambient Water Quality Criteria for the Protection of Human Health (2000) EPA-822-R-09-008; 2009.

3 . Sandermann, H., Jr., Differential lipid affinity of xenobiotics and natural compounds. Febs Lett 2003, 554, (1-2), 165-8.

4. Jabusch, T. W.; Swackhamer, D. L., Partitioning of polychlorinated biphenyls in octanol/water, triolein/water, and membrane/water systems. Chemosphere 2005, 60, (9), 1270-8.

5. Maenpaa, K.; Leppanen, M. T.; Figueiredo, K.; Tigistu-Sahle, F.; Kakela, R., Sorptive capacity of membrane lipids, storage lipids, and proteins: a preliminary study of partitioning of organochlorines in lean fish from a PCB-contaminated freshwater lake. Arch Environ Contam Toxicol 2015, 68, (1), 193-203.

6. Meylan, W. M.; Howard, P. H.; Boethling, R. S.; Aronson, D.; Printup, H.; Gouchie, S., Improved method for estimating bioconcentration/bioaccumulation factor from octanol/water partition coefficient. Environ Toxicol Chem 1999, 18, (4), 664-672.

7. van der Heijden, S. A.; Jonker, M. T., Evaluation of liposome-water partitioning for predicting bioaccumulation potential of hydrophobic organic chemicals. Environ Sci Technol 2009, 43, (23), 8854-9.

8. Sikkema, J.; de Bont, J. A.; Poolman, B., Mechanisms of membrane toxicity of hydrocarbons. Microbiol Rev 1995, 59, (2), 201-22.

9. Konemann, H., Quantitative structure-activity relationships in fish toxicity studies. Part 1: relationship for 50 industrial pollutants. Toxicology 1981, 19, (3), 209-21.

10. Vaes, W. H. J.; Urrestarazu Ramos, E.; Verhaar, H. J. M.; Hermens, J. L. M., Acute toxicity of nonpolar versus polar narcosis: is there a difference? Environ Toxicol Chem 1998, 17, (7), 1380.

11. Yeagle, P., The membrane of cells. 2nd Edition ed.; Academic Press Gennis, R. B. : Boston, 1993.

12. Gobas, F. A.; Lahittete, J. M.; Garofalo, G.; Shiu, W. Y.; Mackay, D., A novel method for measuring membrane-water partition coefficients of hydrophobic organic chemicals: comparison with 1-octanol-water partitioning. J Pharm Sci 1988, 77, (3), 265-72.

13. Dulfer, W. J.; Govers, H. A., Membrane-water partitioning of polychlorinated biphenyls in small unilamellar vesicles of four saturated phosphatidylcholines. Environ Sci Technol 1995, 29, (10), 2548-54.

14. Endo, S.; Escher, B. I.; Goss, K. U., Capacities of membrane lipids to accumulate neutral organic chemicals. Environ Sci Technol 2011, 45, (14), 5912-21.

15. Qu, X.; Wang, X.; Zhu, D., The partitioning of PAHs to egg phospholipids facilitated by copper and proton binding via cation-pi interactions. Environ Sci Technol 2007, 41, (24), 8321-7.

16. Jonker, M. T.; Van der Heijden, S. A., Bioconcentration factor hydrophobicity cutoff: an artificial phenomenon reconstructed. Environ Sci Technol 2007, 41, (21), 7363-9.

17. vanWezel, A. P.; Cornelissen, G.; vanMiltenburg, J. K.; Opperhuizen, A., Membrane burdens of chlorinated benzenes lower the main phase transition temperature in dipalmitoylphosphatidylcholine vesicles: Implications for toxicity by narcotic chemicals. Environ Toxicol Chem 1996, 15, (2), 203-212. 
18. Vaes, W. H.; Ramos, E. U.; Hamwijk, C.; van Holsteijn, I.; Blaauboer, B. J.; Seinen, W.; Verhaar, H. J.; Hermens, J. L., Solid phase microextraction as a tool to determine membrane/water partition coefficients and bioavailable concentrations in in vitro systems. Chem Res Toxicol 1997, 10, (10), 1067-72.

19. Garrett, R. G., C.M., Biochemistry. 2nd ed.

20. Arey, J. S.; Nelson, R. K.; Xu, L.; Reddy, C. M., Using comprehensive two-dimensional gas chromatography retention indices to estimate environmental partitioning properties for a complete set of diesel fuel hydrocarbons. Anal Chem 2005, 77, (22), 7172-82.

21. Wan, Y. S.; Mackay, D., A critical review of aqueous solubilities, vapor pressures, Henry law constants, and octanol-water partition coefficients of the polychlorinated biphenyls. $J$ Phys Chem Ref Data 1986, 15, (2), 911-929.

22. Abraham, M. H.; Poole, C. F.; Poole, S. K., Classification of stationary phases and other materials by gas chromatography. J Chromatogr A 1999, 842, (1-2), 79-114.

23. Breitbach, Z. S.; Armstrong, D. W., Characterization of phosphonium ionic liquids through a linear solvation energy relationship and their use as GLC stationary phases. Analytical and bioanalytical chemistry 2008, 390, (6), 1605-17.

24. Poole, C. F., The Essence of Chromatography. Elsevier: Boston, 2003.

25. Abraham, M. H.; Al-Hussaini, A. J., Solvation parameters for the 209 PCBs: calculation of physicochemical properties. J Environ Monit 2005, 7, (4), 295-301.

26. Hermens, J. L., Quantitative structure-activity relationships of environmental pollutants. In Handbook of Environmental Chemistry, Hutzinger, O., Ed. Springer-Verlag: Berlin, Germany, 1989; Vol. 2E, pp 111-162.

27. McGrath, J. A.; Parkerton, T. E.; Hellweger, F. L.; Di Toro, D. M., Validation of the narcosis target lipid model for petroleum products: Gasoline as a case study. Environ Toxicol Chem 2005, 24, (9), 2382-2394. 


\section{Supporting information for:}

\section{Chapter 2. Estimating phospholipid membrane-water partition coefficients using comprehensive two-dimensional gas chromatography}

\section{S1. Selection of Training Set Compounds}

Initially, our training set included 11 more PCBs (Table S2) with $K_{\text {PLW }}$ values taken from Dulfer and Govers ${ }^{1}$. However, several studies measured the partition coefficients for some of the PCBs in this data set $^{1}$ and found consistently higher values (congeners $\# 61, \# 128$ and $\# 155^{2}$, congeners $\# 136^{3}$ ). In addition, the values of $K_{\mathrm{PLW}}$ from Dulfer and Govers seems to decrease with $\log K_{\mathrm{OW}}$ larger than 6.5 , which is an indication of experimental artifact ${ }^{4}$. Lastly, a recent study on the solvation properties of lipid membranes on 131 compounds $^{3}$ revealed that the $K_{\mathrm{PLW}}$ for the PCBs in Dulfer and Govers ${ }^{1}$ were consistent outliers in this model and concluded that the values were artificially low. ${ }^{3}$ Given all this evidence, we decided to use a more restricted PCB data set, with $K_{\mathrm{PLWS}}$ taken from the compilation of literature values from Endo et al. ${ }^{3}$, as well as another data set from Jabusch and Schwackhamer ${ }^{2}$ (Table 1).

The additional values of $K_{\text {PLW }}$ of PCBs measured by Jabusch and Swackhammer ${ }^{2}$ had not been considered when developing polyparameter model of Endo et al., ${ }^{3}$ and thus we performed an independent multiple linear regression analysis to check for its consistency. We constructed a ppLFER using the descriptor values and $K_{\text {PLW }}$ data of Endo et al., ${ }^{3}$ but excluding all PCBs. We then applied the coefficients to the PCB data set from Jabusch and Scwackhamer. ${ }^{2}$ We found that for the PCB data set used by Endo et al., ${ }^{3}$ the average deviation was less than 2 times the standard error of the regression ( $\mathrm{SE}=0.3$, deviations $<0.6$ ), whereas for the data set of Jabusch and Scwackhamer ${ }^{2}$, the errors were higher, but for 9 out of 11 PBCs, the error was within 3 times the standard error ( errors $<0.9$ ). Thus it appears that there is a difference between the two data sets, but this was not considered sufficient evidence to discard one or the other data set. First, the training set of the ppLFER model consists largely of compounds with $\log K_{\mathrm{PLW}}$ smaller than 5, whereas 
PCBs have $\log K_{\text {PLW }}$ values of at least 5 , which makes the ppLFER not as well constrained at high $\log K$ values. Secondly, the difference between the data sets can also arise from differences in experimental conditions (different lipids used). Given these considerations, we decided to use PCBs from both data sets, and where overlap existed,

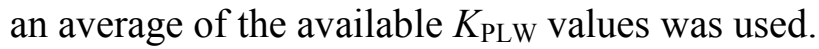


Table S1. Training set used for $\log K_{\mathrm{OW}}$ regression against the $\mathrm{GC} \times \mathrm{GC}$ two-dimension retention times.

\begin{tabular}{|c|c|c|c|c|}
\hline Compound & $\begin{array}{c}\text { First } \\
\text { dimension } \\
\text { retention } \\
\text { time (min) }\end{array}$ & $\begin{array}{l}\text { Second } \\
\text { dimension } \\
\text { retention } \\
\text { time (s) }\end{array}$ & $\begin{array}{c}\text { Experimental } \\
\log K_{\mathrm{OW}}\end{array}$ & $\begin{array}{c}\mathrm{GC} \times \mathrm{GC} \\
\text { predicted } \\
\log K_{\mathrm{OW}}\end{array}$ \\
\hline$p$-xylene & 8.57 & 1.09 & $3.15^{\mathrm{a}}$ & 3.28 \\
\hline naphthalene & 17.17 & 2.09 & $3.30^{\mathrm{b}}$ & 3.38 \\
\hline acenaphthylene & 24.27 & 2.51 & $4.00^{\mathrm{b}}$ & 3.99 \\
\hline acenaphthene & 25.07 & 2.42 & $3.92^{\mathrm{b}}$ & 4.24 \\
\hline fluorene & 27.47 & 2.42 & $4.18^{\mathrm{b}}$ & 4.63 \\
\hline phenanthrene & 31.77 & 2.90 & $4.46^{\mathrm{b}}$ & 4.71 \\
\hline anthracene & 31.97 & 2.86 & $4.45^{\mathrm{b}}$ & 4.79 \\
\hline fluoranthene & 37.37 & 3.14 & $5.16^{\mathrm{b}}$ & 5.31 \\
\hline pyrene & 38.27 & 3.44 & $4.88^{b}$ & 5.06 \\
\hline benz $[a]$ anthracene & 43.97 & 3.52 & $5.79^{\mathrm{b}}$ & 5.90 \\
\hline chrysene & 44.07 & 3.67 & $5.73^{b}$ & 5.72 \\
\hline benzo $[k]$ lfluoranthene & 48.77 & 3.79 & $5.78^{\mathrm{c}}$ & 6.33 \\
\hline benzo $[a]$ pyrene & 49.87 & 4.19 & $6.35^{\mathrm{c}}$ & 5.98 \\
\hline dibenz $[a, h]$ anthracene & 54.07 & 4.28 & $6.75^{\mathrm{d}}$ & 6.55 \\
\hline benzo $[g, h, i]$ perylene & 54.87 & 4.61 & $6.63^{d}$ & 6.25 \\
\hline 3,5-dichlorobiphenyl (\#14) & 30.77 & 2.04 & $5.37^{\mathrm{a}}$ & 5.68 \\
\hline 2,4,6-trichlorobiphenyl (\#30) & 31.37 & 2.06 & $5.71^{\mathrm{a}}$ & 5.76 \\
\hline 4,4'-dichlorobiphenyl (\#15) & 31.87 & 2.34 & $5.58^{\mathrm{a}}$ & 5.47 \\
\hline 2,4,5-trichlorobiphenyl (\#29) & 33.27 & 2.13 & $5.90^{\mathrm{a}}$ & 5.98 \\
\hline 2,4',5-trichlorobiphenyl (\#31) & 33.67 & 2.25 & $5.67^{\mathrm{e}}$ & 5.88 \\
\hline 2,2',4,5-tetrachlorobiphenyl (\#49) & 35.37 & 2.19 & $6.26^{\mathrm{a}}$ & 6.24 \\
\hline 2,2',3,5'-tetrachlorobiphenyl (\#44) & 35.87 & 2.42 & $5.75^{\mathrm{e}}$ & 6.02 \\
\hline 2,2',3,3'-tetrachlorobiphenyl (\#40) & 36.67 & 2.59 & $6.18^{\mathrm{a}}$ & 5.93 \\
\hline 2,3,4,5-tetrachlorobiphenyl (\#61) & 37.37 & 2.28 & $6.41^{\mathrm{a}}$ & 6.45 \\
\hline 2,2',4,4',6,6-hexachlorobiphenyl (\#155) & 38.27 & 2.08 & $7.29^{\mathrm{a}}$ & 6.87 \\
\hline 2,3,4,5,6-pentachlorobiphenyl (\#116) & 39.57 & 2.30 & $6.76^{\mathrm{a}}$ & 6.79 \\
\hline 2,2',3,3’,6,6'-hexachlorobiphenyl (\#136) & 39.67 & 2.56 & $7.12^{\mathrm{a}}$ & 6.46 \\
\hline 2,3,3’,4’,6-pentachlorobiphenyl (\#110) & 39.77 & 2.43 & $6.48^{\mathrm{e}}$ & 6.65 \\
\hline 2,2',3,5,5',6-hexachlorobiphenyl (\#151) & 40.37 & 2.21 & $6.64^{\mathrm{e}}$ & 7.04 \\
\hline 2,2’,3,4,5,5'-hexachlorobiphenyl (\#141) & 42.17 & 2.26 & $6.82^{\mathrm{e}}$ & 7.27 \\
\hline 2,2',3,3’,4,4'-hexachlorobiphenyl (\#128) & 43.47 & 2.68 & $7.32^{\mathrm{a}}$ & 6.93 \\
\hline 2,2',3,4,4',5,5'-heptachlorobiphenyl (\#180) & 45.07 & 2.27 & $8.04^{\mathrm{a}}$ & 7.74 \\
\hline 2,2',3,3’,4,5,5’,6-octachlorobiphenyl (\#198) & 46.37 & 2.20 & $8.38^{\mathrm{a}}$ & 8.04 \\
\hline 2,2',3,3',4,4',5,5,6,6'-decachlorobiphenyl (\#209) & 50.67 & 2.35 & $8.27^{\mathrm{a}}$ & 8.55 \\
\hline 1,3-dichlorobenzene & 12.07 & 1.51 & $3.53^{\mathrm{a}}$ & 3.30 \\
\hline 1,4-dichlorobenzene & 12.27 & 1.56 & $3.44^{\mathrm{a}}$ & 3.27 \\
\hline 1,2-dichlorobenzene & 12.87 & 1.66 & $3.43^{\mathrm{a}}$ & 3.24 \\
\hline 1,2,4-trichlorobenzene & 16.97 & 1.74 & $4.05^{\mathrm{a}}$ & 3.81 \\
\hline
\end{tabular}


1,2,3-trichlorobenzene

1,2,4,5-tetrachlorobenzene

pentachlorobenzene

${ }^{\mathrm{a}}$ ref. ${ }^{5}{ }^{\mathrm{b}}$ ref. ${ }^{6}{ }^{\mathrm{c}}$ ref. ${ }^{7}$ def. ${ }^{3}{ }^{\mathrm{e}}$ ref ${ }^{8}$
17.97

21.27

26.07
1.89

1.75

1.90 $4.14^{\mathrm{a}}$

$4.60^{\mathrm{a}}$

$5.18^{\mathrm{a}}$
3.77

4.50

5.09 
Table S2. Available $K_{\mathrm{PLW}}$ data considered for developing the $\log K_{\mathrm{PLW}}$ versus retention times correlation.

\begin{tabular}{|c|c|c|c|}
\hline Compound & $\begin{array}{c}\text { First } \\
\text { dimension } \\
\text { retention } \\
\text { time (min) }\end{array}$ & $\begin{array}{l}\text { Second } \\
\text { dimension } \\
\text { retention } \\
\text { time }(\mathrm{sec})\end{array}$ & $\begin{array}{c}\text { Experimental } \\
\log K_{\mathrm{PLW}}{ }^{\mathrm{a}}\end{array}$ \\
\hline 2-chlorobiphenyl (\#1) & 25.467 & 2.189 & 4.83 \\
\hline 3,5-dichlorobiphenyl (\#14) & 30.767 & 2.040 & $4.52^{\mathrm{b}}$ \\
\hline 2,4,6-trichlorobiphenyl (\#30) & 31.367 & 2.060 & $4.71^{\mathrm{b}}$ \\
\hline 4,4'-dichlorobiphenyl (\#15) & 31.867 & 2.340 & $4.78^{\mathrm{b}}$ \\
\hline 2,4,5-trichlorobiphenyl (\#29) & 33.267 & 2.130 & $5.16^{\mathrm{b}}$ \\
\hline 2,2',5,5'-tetrachlorobiphenyl (\#52) & 35.167 & 2.220 & 6.12 \\
\hline 2,2',4,5-tetrachlorobiphenyl (\#49) & 35.367 & 2.190 & $5.55^{\mathrm{b}}$ \\
\hline 2,2',3,3'-tetrachlorobiphenyl (\#40) & 36.667 & 2.590 & $5.40^{\mathrm{b}}$ \\
\hline 2,3,4,5-tetrachlorobiphenyl (\#61) & 37.367 & 2.280 & $6.30^{\mathrm{c}}$ \\
\hline 2,2',4,4',6,6'-hexachlorobiphenyl (\#155) & 38.267 & 2.080 & $6.82^{\mathrm{c}}$ \\
\hline 2,3,4,5,6-pentachlorobiphenyl (\#116) & 39.567 & 2.300 & $5.90^{\mathrm{b}}$ \\
\hline 2,2',3,3',6,6'-hexachlorobiphenyl (\#136) & 39.667 & 2.560 & $6.20^{\mathrm{d}}$ \\
\hline 2,2',3,3',4,4'-hexachlorobiphenyl (\#128) & 43.467 & 2.680 & $6.09^{\mathrm{b}}$ \\
\hline 2,2',3,4,4',5,5'-heptachlorobiphenyl (\#180) & 45.067 & 2.270 & $6.33^{\mathrm{b}}$ \\
\hline $2,2^{\prime}, 3,3^{\prime}, 4,5,5^{\prime}, 6$-octachlorobiphenyl (\#198) & 46.367 & 2.200 & $6.18^{\mathrm{b}}$ \\
\hline 2,2',3,3’,4,4',5,5,6,6'-decachlorobiphenyl (\#209) & 50.667 & 2.350 & $6.17^{\mathrm{b}}$ \\
\hline naphthalene & 17.167 & 2.088 & 3.38 \\
\hline phenanthrene & 31.767 & 2.896 & 4.91 \\
\hline anthracene & 31.967 & 2.862 & 5.04 \\
\hline fluoranthene & 37.367 & 3.143 & 5.68 \\
\hline pyrene & 38.267 & 3.440 & 5.48 \\
\hline benz $[a]$ anthracene & 43.967 & 3.520 & 6.53 \\
\hline chrysene & 44.067 & 3.669 & 6.49 \\
\hline benzo $[b]$ fluoranthene & 48.767 & 3.793 & 7.23 \\
\hline benzo $[k]$ fluoranthene & 48.767 & 3.793 & 7.24 \\
\hline benzo $[a]$ pyrene & 49.867 & 4.191 & 7.37 \\
\hline dibenz $[a, h]$ anthracene & 54.067 & 4.279 & 7.80 \\
\hline indeno $[1,2,3-c d]$ pyrene & 54.067 & 4.279 & 7.97 \\
\hline benzo $[g, h, i]$ perylene & 54.867 & 4.606 & 7.91 \\
\hline chlorobenzene & 7.867 & 1.200 & 2.91 \\
\hline 1,3-dichlorobenzene & 12.067 & 1.510 & 3.71 \\
\hline 1,4-dichlorobenzene & 12.267 & 1.560 & 3.57 \\
\hline 1,2-dichlorobenzene & 12.867 & 1.660 & 3.49 \\
\hline 1,2,4-trichlorobenzene & 16.967 & 1.740 & 4.20 \\
\hline 1,2,3-trichlorobenzene & 17.967 & 1.890 & 4.08 \\
\hline 1,2,4,5-tetrachlorobenzene & 21.267 & 1.750 & 4.49 \\
\hline pentachlorobenzene & 26.067 & 1.900 & 5.06 \\
\hline hexachlorobenzene & 30.567 & 1.980 & 5.56 \\
\hline$p$-xylene & 8.567 & 1.090 & 2.98 \\
\hline
\end{tabular}




\begin{tabular}{|c|c|c|c|}
\hline aniline & 11.167 & 2.040 & 1.63 \\
\hline nitrobenezene & 13.967 & 2.290 & 1.96 \\
\hline $\mathrm{N}, \mathrm{N}$-dimethylaniline & 14.467 & 1.810 & 2.33 \\
\hline 2-nitrotoluene & 16.167 & 2.200 & 2.41 \\
\hline 2-allylphenol & 17.267 & 1.890 & 3.06 \\
\hline quinoline & 18.467 & 2.570 & 1.67 \\
\hline 4-chloro-3-methylphenol & 20.067 & 2.040 & 3.34 \\
\hline$m$-nitroaniline & 24.367 & 3.603 & 2.17 \\
\hline 4-n-pentylphenol & 24.467 & 1.770 & 4.31 \\
\hline
\end{tabular}
set.

${ }^{\mathrm{b}}$ ref. $^{l}$

caverage from ref. ${ }^{1}$ and ${ }^{2}$.

$\mathrm{d}_{\text {average from ref. }}{ }^{1}$ and ${ }^{3}$. 
Table S3. Estimated $K_{\text {PLW }}$ using the GC $\times$ GC-based method and the polyparameter (pp) model for compounds with no experimentally determined $K_{\text {PLW. }}$

\begin{tabular}{|c|c|c|c|}
\hline Compound & $\begin{array}{c}\text { Predicted } \\
K_{\mathrm{PLW}} \text { from } \\
\mathrm{GC} \times \mathrm{GC}\end{array}$ & $\begin{array}{l}\text { Predicted } \\
\text { from pp- } \\
\text { model }\end{array}$ & Difference \\
\hline 2,2',5-trichlorobiphenyl (\#18) & 5.87 & $5.75^{\mathrm{a}}$ & 0.12 \\
\hline 2,4',5-trichlorobiphenyl (\#31) & 6.30 & $5.86^{\mathrm{a}}$ & 0.44 \\
\hline 2,2',3,5'-tetrachlorobiphenyl (\#44) & 6.52 & $6.27^{\mathrm{a}}$ & 0.25 \\
\hline 2,3',4,4'-tetrachlorobiphenyl (\#66) & 7.04 & $6.37^{\mathrm{a}}$ & 0.67 \\
\hline 2,2',4,5,5'-pentachlorobiphenyl (\#101) & 7.44 & $6.77^{\mathrm{a}}$ & 0.67 \\
\hline 2,2',3,4,5'-pentachlorobiphenyl (\#87) & 7.29 & $6.77^{\mathrm{a}}$ & 0.52 \\
\hline 2,3,3',4',6-pentachlorobiphenyl (\#110) & 7.31 & $6.77^{\mathrm{a}}$ & 0.54 \\
\hline $2,2^{\prime}, 3,5,5^{\prime}, 6$-hexachlorobiphenyl (\#151) & 7.75 & $7.27^{\mathrm{a}}$ & 0.47 \\
\hline 2,2',4,4',5,5'-hexachlorobiphenyl (\#153) & 8.15 & $7.28^{\mathrm{a}}$ & 0.87 \\
\hline 2,2',3,4,5,5'-hexachlorobiphenyl (\#141) & 8.05 & $7.29^{\mathrm{a}}$ & 0.76 \\
\hline 2,2',3,4,4',5'-hexachlorobiphenyl (\#138) & 7.92 & $7.28^{\mathrm{a}}$ & 0.64 \\
\hline $2,2^{\prime}, 3,4^{\prime}, 5,5^{\prime}, 6$-heptachlorobiphenyl (\#187) & 8.43 & $7.78^{\mathrm{a}}$ & 0.65 \\
\hline 2,2',3,4,4',5,5'-heptachlorobiphenyl (\#180) & 8.64 & $7.76^{\mathrm{a}}$ & 0.87 \\
\hline 2,2',3,3',4,4',5-heptachlorobiphenyl (\#170) & 8.43 & $7.80^{\mathrm{a}}$ & 0.63 \\
\hline$\alpha-\mathrm{HCH}$ & 5.29 & $4.49^{\mathrm{b}}$ & 0.80 \\
\hline$\beta-\mathrm{HCH}$ & 4.57 & $4.08^{\mathrm{b}}$ & 0.48 \\
\hline$\gamma-\mathrm{HCH}$ & 5.24 & $4.34^{\mathrm{b}}$ & 0.90 \\
\hline heptachlor & 6.10 & $5.81^{\mathrm{c}}$ & 0.29 \\
\hline aldrin & 6.95 & $6.43^{\mathrm{c}}$ & 0.53 \\
\hline heptachlor epoxide & 7.19 & $5.47^{\mathrm{c}}$ & 1.72 \\
\hline$\alpha$-endosulfan & 7.06 & $5.05^{\mathrm{c}}$ & 2.01 \\
\hline 4,4'-DDE & 7.25 & $7.09^{\mathrm{c}}$ & 0.17 \\
\hline dieldrin & 7.54 & $5.01^{\mathrm{c}}$ & 2.53 \\
\hline endrin & 7.36 & $4.64^{\mathrm{c}}$ & 2.72 \\
\hline 4,4'-DDD & 6.76 & $7.44^{\mathrm{c}}$ & -0.68 \\
\hline 4,4'-DDT & 7.79 & $7.30^{\mathrm{c}}$ & 0.49 \\
\hline methoxychlor & 7.52 & $5.04^{\mathrm{c}}$ & 2.48 \\
\hline
\end{tabular}

Solute descriptors were taken from ${ }^{\mathrm{a}}$ ref. ${ }^{9}$. ${ }^{\mathrm{ref}} .{ }^{10}$. ${ }^{\mathrm{c}}$ ref. ${ }^{11}$. 

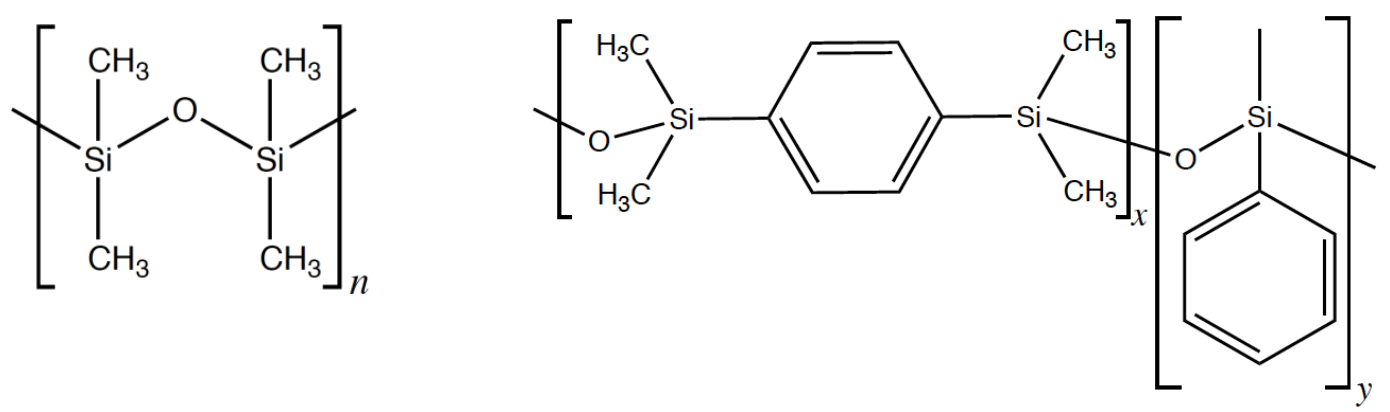

Figure S1. Stationary phases used in the $\mathrm{GC} \times \mathrm{GC}$ : polydimethyl siloxane, first dimension column stationary phase (left), and $50 \%$ phenyl polysilphenylene-siloxane, the second dimension column stationary phase (right). 


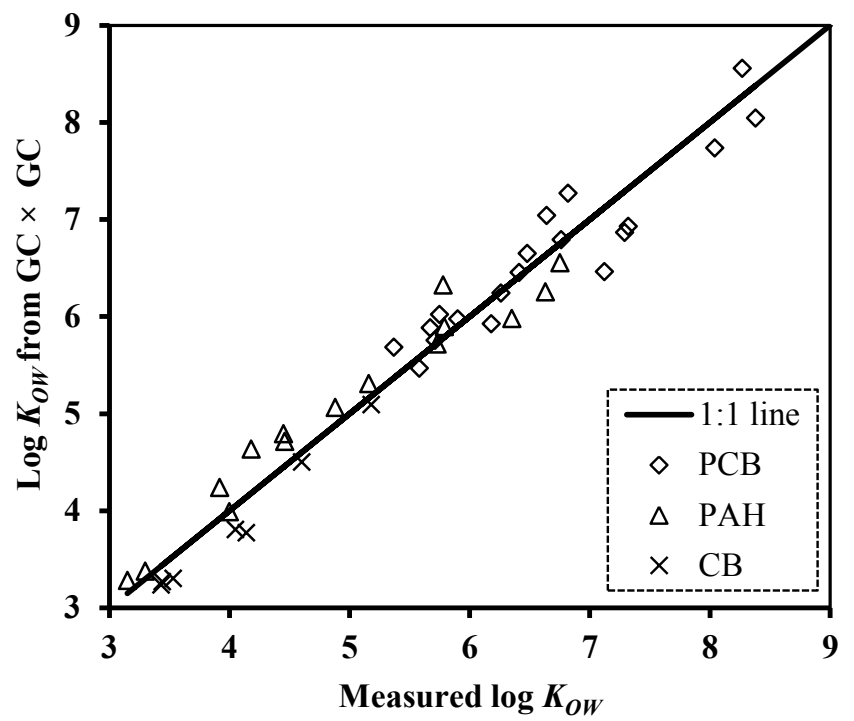

Figure S2. Measured $\log K_{\mathrm{Ow}}$ versus estimated $\log K_{\mathrm{OW}}$ from $\mathrm{GC} \times \mathrm{GC}$ retention behavior for a training set of apolar and monopolar chemicals. 

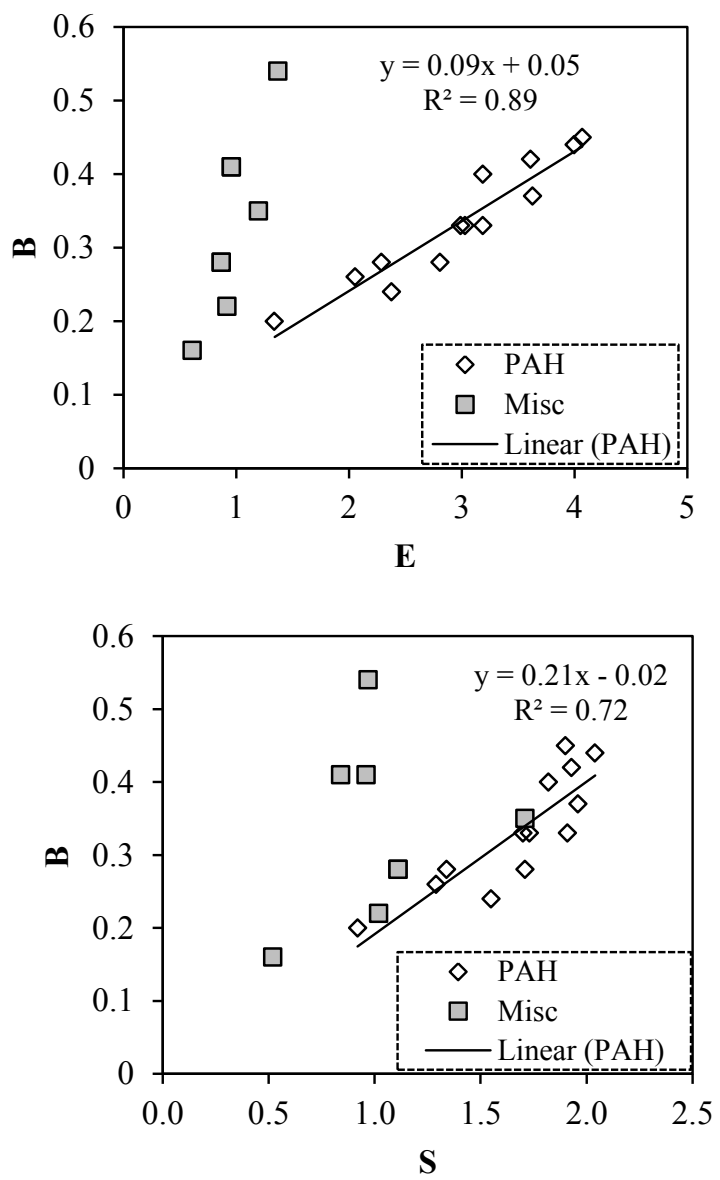

Figure S3. Correlation of $\mathrm{B}$ versus $\mathrm{E}$ and $\mathrm{S}$ for compounds with $\mathrm{B}>0.2$ in the training set. Solute descriptors were taken from ref. ${ }^{3}$ with the exception of naphthalene. ${ }^{12}$ 
Reference

1. Dulfer, W. J.; Govers, H. A., Membrane-water partitioning of polychlorinated biphenyls in small unilamellar vesicles of four saturated phosphatidylcholines. Environ Sci Technol 1995, 29, (10), 2548-54.

2. Jabusch, T. W.; Swackhamer, D. L., Partitioning of polychlorinated biphenyls in octanol/water, triolein/water, and membrane/water systems. Chemosphere 2005, 60, (9), 1270-8.

3. Endo, S.; Escher, B. I.; Goss, K. U., Capacities of membrane lipids to accumulate neutral organic chemicals. Environ Sci Technol 2011, 45, (14), 5912-21.

4. Jonker, M. T. O.; van der Heijden, S. A., Bioconcentration factor hydrophobicity cutoff: An artificial phenomenon reconstructed. Environ Sci Technol 2007, 41, (21), 7363-7369.

5. Patel, H.; Schultz, T. W.; Cronin, M. T. D., Physico-chemical interpretation and prediction of the dimyristoyl phosphatidyl choline-water partition coefficient. $J \mathrm{Mol}$ Struc-Theochem 2002, 593, 9-18.

6. Arey, J. S.; Nelson, R. K.; Xu, L.; Reddy, C. M., Using comprehensive twodimensional gas chromatography retention indices to estimate environmental partitioning properties for a complete set of diesel fuel hydrocarbons. Anal Chem 2005, 77, (22), 7172-82.

7. Fernandez, L. A.; MacFarlane, J. K.; Tcaciuc, A. P.; Gschwend, P. M., Measurement of freely dissolved PAH concentrations in sediment beds using passive sampling with low-density polyethylene strips. Environ Sci Technol 2009, 43, (5), 14301436.

8. Smedes, F.; Geertsma, R. W.; van der Zande, T.; Booij, K., Polymer-water partition coefficients of hydrophobic compounds for passive sampling: application of cosolvent models for validation. Environ Sci Technol 2009, 43, (18), 7047-54.

9. Abraham, M. H.; Al-Hussaini, A. J., Solvation parameters for the 209 PCBs: calculation of physicochemical properties. J Environ Monit 2005, 7, (4), 295-301.

10. Goss, K. U.; Arp, H. P. H.; Bronner, G.; Niederer, C., Partition behavior of hexachlorocyclohexane isomers. Journal of Chemical and Engineering Data 2008, 53, (3), 750-754.

11. Hale, S. E.; Martin, T. J.; Goss, K.-U.; Arp, H. P. H.; Werner, D., Partitioning of organochlorine pesticides from water to polyethylene passive samplers. Environ Pollut 2010, 158, (7), 2511-2517.

12. Burns, S. T.; Khaledi, M. G., Rapid determination of liposome-water partition coefficients (K-lw) using liposome electrokinetic chromatography (LEKC). J Pharm Sci 2002, 91, (7), 1601-1612. 



\section{Chapter 3}

\section{Evaluating bioaccumulation potential of HOC mixtures using GC $\times$ GC and passive sampling}

A. Patricia Tcaciuc ${ }^{1,2}$ *, Robert K. Nelson ${ }^{2}$, Josefin L Betsholtz ${ }^{1}$, Christopher M. Reddy ${ }^{2}$, and Philip M. Gschwend ${ }^{1}$

${ }^{1}$ Department of Civil and Environmental Engineering, Massachusetts Institute of Technology, Cambridge, MA 02139, USA

${ }^{2}$ Department of Marine Chemistry and Geochemistry, Woods Hole Oceanographic Institution, Woods Hole, MA 02543, USA 


\section{Abstract}

Chemical activities of hydrophobic organic compounds (HOCs) in sediments govern the tendency of these chemicals to bioaccumulate in benthic biota and migrate out of sediment beds. Advances in passive sampling have greatly improved our ability to measure freely dissolved concentrations (which are closely related to chemical activities) of HOCs, but challenges remain, particularly for mixtures, as the toxic effects of HOCs can be cumulative, and the identification and quantification of each mixture component may not be feasible. Combining comprehensive two dimensional gas chromatography $(\mathrm{GC} \times \mathrm{GC})$ and polyethylene $(\mathrm{PE})$ passive sampling can help us estimate body burdens of a variety of contaminants from their corresponding accumulation in passive samplers. To this end, we developed a GC $\times$ GC-based relationship for evaluating polyethylene-water partition coefficients $\left(K_{P E W}\right)$. The relationship performed well for a variety of chemicals (16 PAHs, 21 PCB, 12 benzene and cyclohexane derivatives and 5 OCPs), allowing for determination of $K_{P E W}$ values on average to within a factor of $3\left(R^{2}=0.88, S E=\right.$ $0.44 \log$ units). We then combined the relationship for $K_{P E W}$ with previous work relating the phospholipid membrane-water partition coefficients $\left(K_{P L W}\right)$ to $\mathrm{GC} \times \mathrm{GC}$ retention times, and estimated how different classes of HOCs would partition between passive samplers and lipids. Across the GC $\times$ GC space, the lipid-PE partition coefficients varied by two orders of magnitude. PAHs and PCBs preferred lipids over PE (by factors of six for PAHs and 2-3 for PCBs), while saturated hydrocarbons partitioned more into the PE phase (by factors of 3-5). These finding were in agreement with independent estimates based on literature values of $K_{P E W}$ and $K_{P L W}$. We also tested the predictions of the GC $\times$ GC model against observed bioaccumulation of various contaminants by the polychaete Nereis virens and PE. We found that the model performed well for PCBs (agreement within a factor of 2-3), but for all other contaminants (PAHs, DDXs (DDT and its degradation products DDE and DDD), hydrocarbons) investigated, the method overestimated the measured accumulation. For DDXs, the measured accumulation was within a factor of 2 for DDD and 6 for DDE. Metabolic processes affected the behavior of PAHs for which PE-based estimates were 10 - 300 times larger the measured accumulation in the worms. Based on analysis on a GC $\times$ GC coupled with flame ionization detector (FID), the accumulation of various aliphatic and aromatic hydrocarbons (but not condensed aromatic compounds like PAHs) was also between 10-100 times lower in the worms compared to PE. The observed disagreement between the GC $\times$ GC based estimations of lipid-PE partitioning and the measured 
concentrations of contaminants in actual biota probably reflects the impact of metabolic activities on contaminant accumulation. Nonetheless, body burdens calculated based on the assumption of chemical equilibrium between the sediment and the biota (and using $\mathrm{GC} \times \mathrm{GC}$ for evaluating the required partition coefficients), could (1) be useful for defining the upper limit of expected contaminant loads, and (2) serve as a starting point for investigating the conditions (species, contaminants site characteristics) when metabolic transformations and/or metabolic stress may be present. 


\subsection{Introduction}

Contamination of aquatic systems with hydrophobic organic chemicals (HOCs) is of concern due the bioaccumulation potential and toxicity of HOCs. Because of their hydrophobicities, HOCs are often found at high concentrations in sediment beds, where they associated with organic matter and other nonpolar sorptive phases (e.g., black carbon). Sediments can act as a source of HOCs to the overlying water through diffusive fluxes or via food webs. HOCs accumulate in the lipids of benthic organisms, from where they are transferred to benthic predators and higher trophic level fish. As many HOCs have been linked to chronic effects, body burdens of HOCs in fish can have negative effects on human health and are often the basis for fish consumption advisories ${ }^{1}$ or remediation decisions.

Prediction of body burdens from measurements of contaminant concentrations in sediment and in water is a key step in evaluating the hazard of contaminated sediments. Traditional methods have undergone scrutiny because they often did not match empirical observations of HOC accumulation. ${ }^{2-4}$ In one approach, sediment concentrations were used in combination with an equilibrium partitioning model (EqP) to determine corresponding body burdens expected at equilibrium with the sediments. However, the diversity of sorptive phases in sediments, whose abundances and affinities for HOCs are both difficult to measure, lead to uncertain estimations for bioavailability of HOCs, i.e., the fraction of the chemicals available for organism uptake. When only the sorption to organic carbon was considered, the EqP model usually overpredicted the accumulation of HOCs in benthic biota. $^{2,} 4$ In a different approach, bioaccumulation is formulated as the result of differential uptake and removal rates, which leads to steady-state body burdens. ${ }^{5}$ Traditionally, these toxicokinetics approaches rely on previously measured bioconcentration factors (BCF) and/or biota sediment accumulation factors (BSAF), which are the ratio of lipid normalized concentrations in organisms over the concentrations in water and organic carbon normalized concentrations in sediment, respectively. Both approaches can lead to erroneous results when bulk instead of freely dissolved concentrations are used, and researchers and regulatory agencies now recognize that bioaccumulation is more accurately characterized on the basis of freely dissolved concentrations, ${ }^{3,6,7}$ rather than bulk sediment concentrations.

Compared to traditional methods, involving concentration measurements in sediments and porewater, passive sampling offers a more reliable way of measuring the freely dissolved concentration of HOCs in sediments, which could in turn be used to evaluate body burdens. ${ }^{7,8}$ 
Several studies have employed passive sampling in combination with bioaccumulation experiments and found good agreement between passive sampler-based predictions and observations of body burdens in benthic biota. ${ }^{3,4,9-11}$ Although the use of passive sampling is preferred to direct measurement of freely dissolved concentrations, because it is analytically simpler, some challenges remain for the interpretation of passive sampling data. Notably, in an equilibrium approach, the estimated body burdens depend on the measured concentrations in the passive sampler but also on the partition coefficients between sampler-water and lipid-water (Figure 3.1). Even for polymers like polyethylene (PE), that have been in use for more than a decade, the measured PE-water partition coefficient $\left(\mathrm{K}_{\mathrm{PEW}}\right)$ can vary by more than one order of magnitude across different experimental determinations. ${ }^{12}$ Similar issues have been observed for lipid-water partitioning (Chapter 2), which can then lead to errors more than one order of magnitude in predicted body burdens. In addition, the octanol-water partition coefficient is often used a surrogate for the lipid water partition coefficient, which does not capture the fact that the partitioning into different lipid classes (membrane and storage lipids) can vary for different chemicals. $^{13}$ The use of many different polymers (PE, polyoxymethylene (POM), polydimethylsiloxane (PDMS) etc.) adds another layer of variability. While most of the polymers used to measure HOCs are nonpolar, and should have very similar affinities for HOCs, it is known that variations exists in both partitioning and diffusion properties. ${ }^{14}$ For example, relative to the partition coefficient for PDMS-water, the partitioning of PAHs in PE tends to be a $0.5-1$ $\log$ units larger, whereas partitioning into POM tends to be an order of magnitude lower. ${ }^{15}$ Lastly, we acknowledge that some of the variability that exists for $K_{P E W}$ could be due to variation in the PE properties (e.g., different sources, or batches), but this possibility has not been addressed thus far in the passive sampling literature.

For certain classes of HOCs, determination of partition coefficients is challenging due to the diversity of constituents. For hydrocarbon mixtures, such as those derived from petroleum, identification of each component is often not possible using simple gas chromatographic techniques. Although the separation of compounds is improved by using comprehensive twodimensional gas chromatography $(\mathrm{GC} \times \mathrm{GC})$, unresolved complex mixtures $(\mathrm{UCM})$ are still often present in part of the chromatogram. UCM components have been found to accumulate in marine organisms and have been linked to narcosis toxicity. ${ }^{16}$ For such mixtures, it is desirable to have a way to characterize the partitioning of all the mixture components in one step by making 
use of the fact that two dimensional gas chromatography analysis can also give us information on the properties of the chemicals. ${ }^{17}$ Additionally, chromatography-based methods of determining partition coefficients could be applied to chemicals that are usually used and consequently, released in the environment, as technical mixtures, such as PCBs or polychlorinated alkanes. Additionally, chromatography-based methods may be more efficient than direct measurements, because such determinations would only require running the compound(s) on a $\mathrm{GC} \times \mathrm{GC}$ system.

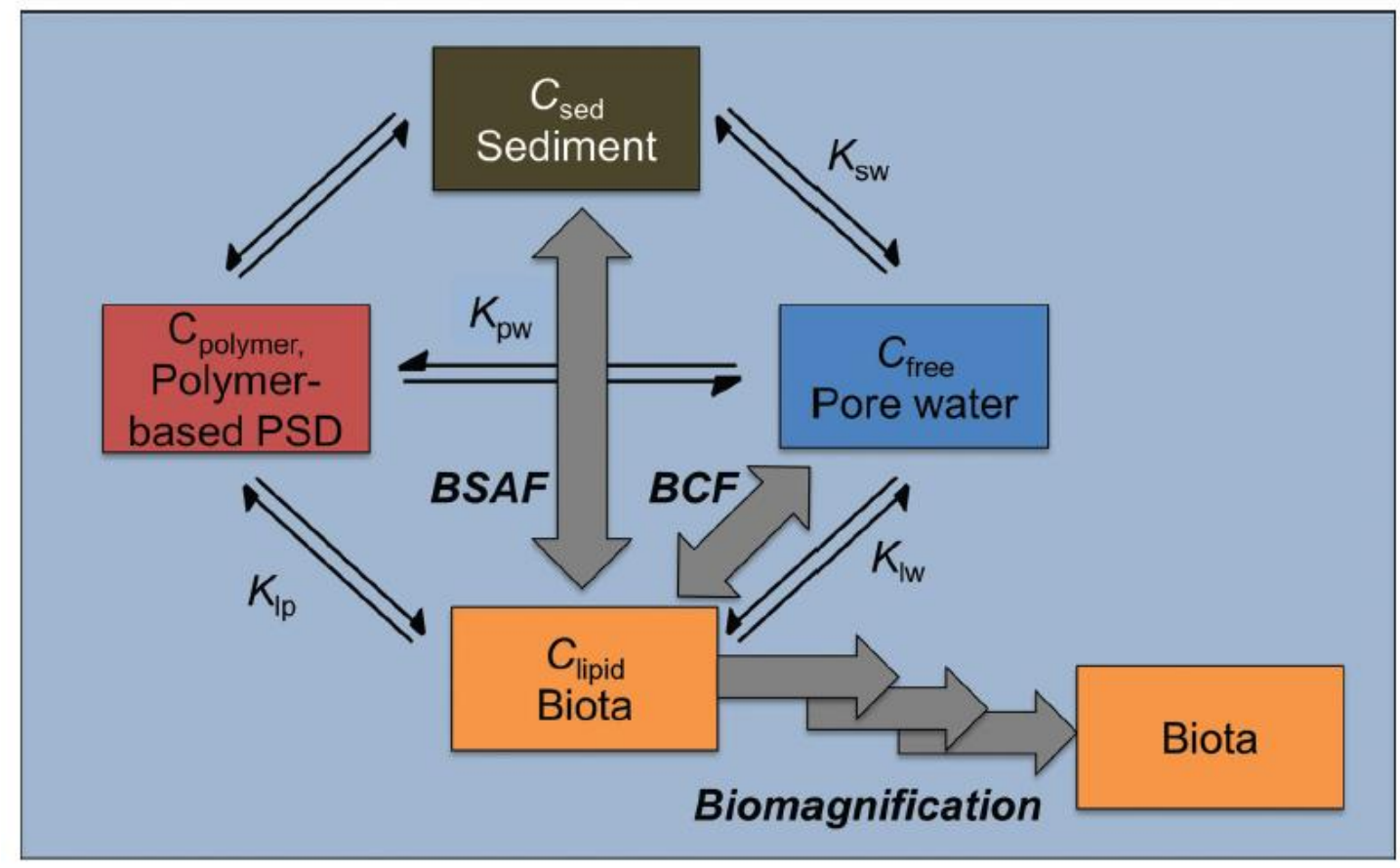

Figure 3.1 (reproduced from Lydy et al. ${ }^{10}$ ) Schematic of relationships that can be used to connect concentrations between various compartments with equilibrium partitioning (two way black arrows) or toxico-kinetic approaches (grey solid arrows). BSAF is the biota sediment accumulation factor equal to the ratio of lipid normalized biota concentration $\left(\mathrm{g} / \mathrm{g}_{\text {lipid }}\right)$ at steady state, divided by organic carbon normalized concentration in the sediment $\left(\mathrm{g} / \mathrm{g}_{\mathrm{OC}}\right)$. BCF is the bioconcentration factor expressed as lipid normalized biota concentration $\left(\mathrm{g} / \mathrm{g}_{\text {lipid }}\right)$ at steady state, and porewater concentration $\left(\mathrm{g} / \mathrm{cm}^{3}{ }_{\mathrm{w}}\right)$. At equilibrium, BCF is equal to the lipid-water partition coefficient ( $K_{l w}$ in the figure, $K_{P L W}$ in text). $K_{l p}$ in figure is $K_{P L-P E}$ in text.

The goal of this work was to use GC $\times$ GC retention time information to understand how various HOCs partition between passive samplers and lipids, in order to evaluate their bioaccumulation potential and aid in interpretation of HOC accumulation in passive samplers. 
We first use a similar approach to that developed in Chapter $2^{18}$ to develop a GC $\times$ GC-based method of estimating PE-water partition coefficient $\left(K_{P E W}\right)$, which we use in combination with $K_{P L W}$ (the phospholipid-water partition coefficient) prediction method developed in Chapter 2 to estimate lipid-polyethylene $\left(K_{P L-P E}\right)$ partition coefficients. Then, using bioaccumulation experiments with Nereis virens in sediments contaminated with various HOCs, we compare the accumulation of HOCs in lipids predicted from passive sampling and GC $\times$ GC against empirical observations of lipid-normalized body burdens in the polychaete Nereis virens. Throughout this manuscript, we assume that the measured body burden of chemicals in the polychaete is all due to partitioning into the lipids, recognizing however, that depending on the sorption capacity of proteins this assumption may lead to an overestimation of lipid normalized concentrations by at most a factor of two. For example, while the sorptive capacity of proteins is lower than that of lipids (e.g., sorptive capacity of proteins in animal tissue 1 to $10 \%$ that of lipids ${ }^{19}$ ) the typical abundance of proteins in Nereis virens is higher than that of lipids $\left(7 \%\right.$, w.w. for proteins ${ }^{20}$ but 1\% w.w for lipids, Figure 3.2), which implies that between 10 and $50 \%$ of the measured body burdens of chemicals could be due to partitioning of chemicals into proteins. In addition, while other classes besides membrane lipids, such as storage lipids, also have high affinities for HOCs, in this manuscript we consider partitioning mainly to membrane lipids, as some studies found that these dominate over storage lipids in mature polychaetes. ${ }^{21,22}$ Lastly, we discuss the GC $\times$ GC based predictions of sampler-lipid partitioning in the context of existing knowledge of the partitioning of various HOC classes, as well as the impact on the bioaccumulation predictions of the assumption made in this study that body burdens are dominated by accumulation in the membrane lipids.

Recent work by Nabi et al. ${ }^{23}$ proposed a new approach for estimating partition coefficients from $\mathrm{GC} \times \mathrm{GC}$ retention information, through the use of retention indices (RI). The RI method is attractive because it can be used to compare samples that were run on different temperature programs. When developing the $K_{P E W}$ relationship, we employed and compared retention time (RT)- and retention index (RI)-based approaches. For consistency, we also revisit the correlation derived in Chapter 2 for $K_{P L W}$, expanding the training set and developing a RI-based correlation.

The set of organic chemicals used in this work and measured in the bioaccumulation experiments were selected (1) to reflect an environmentally relevant set of organic chemicals (e.g., PAHs, PCBs, DDT and its degradation products DDE and DDD) and (2) offer a good 
distribution of training set compounds across the GC $\times$ GC space (because the set of contaminants mentioned above eluted high in the second dimension (between 3 and $5.5 \mathrm{~s}$ ), the training set was expanded to include a series of alkylated benzenes and cyclohexanes with second dimension retention times between 2 and $2.6 \mathrm{~s}$ ). There are over 100 different PAHs which may exist in the environment, but in this study we focus on a set of 16 PAHs (naphthalene, acenaphthene, acenaphthylene, fluorene, phenanthrene, anthracene, fluoranthene, pyrene, benz $[a]$ anthracene, chrysene, benzo $[b]$ fluoranthene, benzo $[k]$ fluoranthene, benzo $[a]$ pyrene, indeno[1,2,3-cd]pyrene, benzo[ghi]perylene, dibenz $[a, h]$ anthracene) which are included on a list of 127 priority pollutants published by the US Environmental Protection Agency. Several PCB technical mixtures, also known as Aroclors, and DDT, DDE and DDD, are also included on the priority pollutants list. In this study, we focus on the quantification of individual congeners (as opposed to Aroclors) and in particular, on a subset of 20 of the possible 209 congeners (also known as the NOAA 20 PCB congeners: ${ }^{24}$ PCB 8, PCB 18, PCB 28, PCB 52, PCB 44, РCB 66, РCB 77, РCB 101, РCB 118, PCB 105, PCB 126, PCB 153, PCB 138, PCB 128, PCB 187, PCB 180, PCB 170, PCB 195, PCB 206, PCB 209). In addition to these, several additional PCB congeners are included in the $\mathrm{GC} \times \mathrm{GC}$ training set as discussed in section 3.2.1 and illustrated in Table S1.

\subsection{Materials and Methods}

We used Ultra-Resi analyzed solvents (dichloromethane, hexane, methanol, all from J.T. Baker) for all extraction procedures. Salts used for making seawater were purchased from Sigma Aldrich: sodium chloride (Macron), calcium chloride dihydrate and potassium bromide (Sigma Aldrich), sodium sulfate (J.T. Baker), boric acid, sodium bicarbonate, magnesium chloride, and potassium chloride (Mallinckrodt). Isotopically labeled PCBs and DDXs, used as recovery and injection standards were purchased from Cambridge Isotope Laboratory (Tewksbury, MA). Isotopically labeled PAHs and standard mixtures of PCBs, DDXs, PAHs, n-alkanes, and chlorobenzenes were purchased from Ultra Scientific (N. Kingstown, RI). Linear alkylbenzenes and alkylcyclohexanes were procured from Chiron ( Trondheim, Norway).

\subsubsection{Selection of $K_{P E W}$ training set.}


$K_{P E W}$ values were compiled from multiple sources for PCBs, ${ }^{14,25-27} \mathrm{PAHs}^{14,}{ }^{25-27}$ and organochlorine pesticides (OCPs). ${ }^{28}$ The geometric mean was used when multiple values were found for the same compound (Table S1). In addition, $K_{P E W}$ values of several hydrocarbons with second dimension retention times between 2 and $2.6 \mathrm{~s}$, were measured as detailed in the following section, to ensure a better distribution of training set compounds across $\mathrm{GC} \times \mathrm{GC}$ space. For PCBs and PAHs, the $K_{P E W}$ values were in general agreement (within $0.3 \log$ units) between the two largest data sets available - Choi et al. ${ }^{25}$ and Smedes et al. ${ }^{14}$ The only exceptions were the three PAHs, indeno[1,2,3-cd]pyrene, dibenz $[a, h]$ anthracene and benzo[ghi]perylene, for which the $K_{P E W}$ values measured by Smedes et al. ${ }^{14}$ were up to $1 \log$ unit larger than those of Choi et al. ${ }^{25}$ This may have been due to an experimental artifact, such as the presence of organic carbon or organic solvent in the water, or losses of the test compounds to the air-water interface, ${ }^{29}$ all of which can lower the measured $K_{P E W}$. In contrast, Smedes et al. ${ }^{14}$ used a co-solvent approach, where the $K_{P E W}$ values were determined by extrapolation from partition coefficients measured in at various solvent percentages in methanol-water-polymer systems. The $K_{P E W}$ values measured by Smedes et al. ${ }^{14}$ for the three above-mentioned PAHs were preferred over the values of Choi et al. ${ }^{25}$, since in solvent-water systems, the solubilities of the chemicals' in the liquid phases are larger than in pure water. This increase in solubility (1) reduces the effect on the measured $K_{P E W}$ of any dissolved organic matter that may be potentially present in the system, and (2) decreases the likelihood that hydrophobic chemicals will prefer the air water interphase. Lastly, while the presence of the methanol could potentially change the activity coefficient of the chemicals in the polymer phase, this is likely to have a small effect on the measured $K_{P E W}$ value since (1) polyethylene swelling is small in methanol $(0.1 \%)^{15}$ and (2) there has been thus far, good agreement between partition coefficients measured using co-solvent systems and using only pure water, for polyethylene (agreement to within $0.3 \log$ units for over 30 PCBs and PAHs, with the exceptions of the three PAHs mentioned above) but also for polymers that are known to swell more in methanol (e.g., $2.5 \%$ swelling of silicone rubbers, but agreement to within $0.3 \mathrm{log}$ units for Altesil 678 polymer-water coefficients of $43 \mathrm{PCB}$ congeners measured both using pure water and the co-solvent method $^{14}$ ).

\subsection{2 $K_{P E W}$ determination for hydrocarbons.}


To ensure a better distribution of compounds across $\mathrm{GC} \times \mathrm{GC}$ retention space, $K_{P E W}$ values were determined experimentally for a series of linear alkylcyclohexanes ( $n \mathrm{ACH}: n$-heptyl, $n$ octyl and $n$-nonylcyclohexane), and a series of linear alkylbenzenes (LAB: $n$-heptyl through $n$ dodecylbenzene). These compounds had second dimension retention times between 2 and $2.6 \mathrm{~s}$, which was an area of the chromatogram that was not covered by the set of PCBs, PAHs and OCPs discusses above (Table S1). Several pieces of $5 \mathrm{mg}$ PE were loaded with the $n \mathrm{ACH}$ mixture or the LAB mixture from a 80:20 methanol:water mixture. One piece of PE was then suspended on a combusted glass Pasteur pipette and placed in a $1 \mathrm{~L}$ amber glass bottle filled with MiliQ water. The experiments were done in duplicate. The bottles were placed on an orbital shaker table and after $1 \mathrm{mo}$, both the PE and water were extracted with dichloromethane. A mixture of biphenyl and $d_{14}-p$-terphenyl were added as recovery standards to each water and PE sample before extraction. There overnight solvent extractions at $21{ }^{\circ} \mathrm{C}$ were performed for PE, and three liquid-liquid extractions were also performed for the water phase using about 1:10 ratio of solvent to water and shaking each extraction for $10 \mathrm{~min}$. The combined extracts were then concentrated using rotary evaporation to a volume of $\sim 0.1-1 \mathrm{~mL}$. Both PE and water extracts were then analyzed using gas chromatography-mass spectrometry (GC-MS, Section 3.2.9). Several pieces of PE loaded with either $n \mathrm{ACH}$ or LAB mixture were analyzed after the loading step to check for mass balance during the desorption experiment, and the concentrations measure for PE and water were corrected by the average recovery of the two surrogate standards (biphenyl and $\mathrm{d}_{14}-p$-terphenyl). Across all PE and water extractions, the average recovery of biphenyl and $\mathrm{d}_{14}$-p-terphenyl were $87 \pm 16$ and $120 \pm 30$, respectively. The recoveries of both biphenyl or $\mathrm{d}_{14}$ - $p$-terphenyl were similar in PE and in the water extractions, and using either recovery compound or the average recovery of both biphenyl or $\mathrm{d}_{14}$ - $p$-terphenyl to correct the measured concentration of LABs or ACHs, did not change the measured $K_{P E W}$ more than $0.1 \log$ units (e.g., ratio of recoveries measured in PE and water of biphenyl and $\mathrm{d}_{14}$-p-terphenyl were 108\% (PE):82\% (water), and 131\% (PE):106\% (water), respectively, which equal 0.76 and 0.80 , respectively). Mass balances at the end of the experiment ranged were $89 \pm 23 \%$ (range 66 to $130 \%$ ), and deviations between duplicate measurements of $K_{P E W}$ values were typically less than $0.2 \log$ units. The only exception was $n$-dodecylbenzene for which one of the duplicates had a low mass balance of $11 \%$, and hence the $K_{P E W}$ measured in this bottle was not considered further. The loss of chemicals to the bottle headspace $(\max 10 \mathrm{~mL})$ was calculated based on the 
compounds' Henry's law constants and was $<10 \%$ for all $n$-LABs and $n$-ACHs, with the exception of $n$-heptylcyclohexane $\left(30 \%, K_{H}\right.$ of $\left.1.83 \mathrm{~atm} \mathrm{~m} / \mathrm{mol}^{30}\right)$.

For hydrocarbons with aqueous solubility greater than $1 \mathrm{mg} / \mathrm{L}$, such as tetralin and $t$ pentylbenzene, $K_{P E W}$ values were determined from measurements of initial and equilibrium aqueous concentrations in a PE uptake experiment. Two $300 \mathrm{~mL}$ bottles, equipped with glass coated stirbars, were spiked with either tetralin or $t$-pentylbenzene at $\sim 25$ and $5 \mathrm{mg} / \mathrm{L}$ concentration, respectively. A piece of PE was added to the experimental flask and the concentration in the water was monitored in the control and experimental flasks, via direct aqueous injection GC - FID analysis on a Carlo Erba HRGC 5300 Mega Series. Injections of 1 $\mu \mathrm{L}$ were performed on-column, on a $30 \mathrm{~m}$ DB-5 MS column $(0.25 \mu \mathrm{m}$ film thickness, $0.25 \mathrm{~mm}$ diameter, Agilent Technologies, Santa Clara, CA) at $100{ }^{\circ} \mathrm{C}$, with a helium carrier gas flow of 3 $\mathrm{mL} / \mathrm{min}$. The oven temperature was ramped from 100 to $250{ }^{\circ} \mathrm{C}$ at $20{ }^{\circ} \mathrm{C} / \mathrm{min}$. For tetralin, $\mathrm{PE} /$ water equilibrium was reached within $1 \mathrm{~d}$ and the concentration in the control flask showed less than $10 \%$ loss compared to the start of the experiment. Neglecting the $10 \%$ loss observed in the control flaks, the concentration of tetralin in $\mathrm{PE}$ at equilibrium was then calculated by difference based on the initial and final concentrations in water in the experimental flask. Finally, $K_{P E W}$ was calculated as the ratio of the concentration in PE over the concentration in water. For $t$-pentylbenzene, the control bottle showed $\sim 15 \%$ loss at the end of the experiment ( $4 \mathrm{~d}$ ), so the $\mathrm{PE}$ concentration was calculated from measured aqueous concentration in the experimental flask adjusted by the loss observed in the control flask $\left(\mathrm{C}^{\mathrm{t}=4 \mathrm{~d}}{ }_{\mathrm{PE}}=\mathrm{V}_{\mathrm{W}} / \mathrm{m}_{\mathrm{PE}}\left[\mathrm{C}^{\mathrm{t}=0}\right.\right.$ experimental-flask $\mathrm{C}_{\text {control- }}^{\mathrm{t}=4 \mathrm{~d}}$ flask $/ \mathrm{C}^{\mathrm{t}=0}$ control-flask $-\mathrm{C}^{\mathrm{t}=4 \mathrm{~d}}$ experimental-flask flask]) before calculating the concentration of chemical in PE and $K_{P E W}\left(\mathrm{C}^{\mathrm{t}=4 \mathrm{~d}}{ }_{\mathrm{PE}} / \mathrm{C}^{\mathrm{t}=4 \mathrm{~d}}{ }_{\mathrm{W}}\right)$.

\subsubsection{Development of RT and RI-based correlations.}

Known PE-water partition coefficients of the compounds in the training set (Table S1) were regressed against retention time $(T)$ using multi linear regression in StatPlus package to obtain a relationship of the form:

$$
\log K_{P E W}=a T_{1}+b T_{2}+c
$$

where $T_{1}$ and $T_{2}$ were the retention times on the first and second dimensions respectively. 
Similarly, for developing the RI-based correlation, ${ }^{23}$ the $K_{P E W}$ values were regressed against $u_{1}$ and $u_{2}$, the first and second dimension retention vectors (an orthogonal version of retention indices, see below), respectively:

$$
\log K_{P E W}=a^{\prime} u_{1}+b^{\prime} u_{2}+c^{\prime}
$$

Following the procedure laid out by Nabi et al., ${ }^{23}$ in order to evaluate $u_{1}$ and $u_{2}$, we first calculated the stationary phase-gas partition coefficients, $L_{1}$ and $L_{2}$, at $120{ }^{\circ} \mathrm{C}$ for a set of 56 chemicals (Table S2), based on the Abraham solute model (ASM) characterization of the two stationary phases:

$$
\log L=e E+s S+a A+b B+l L+c
$$

where $E, S, A, B$, and $L$ are the Abraham model solute descriptors capturing excess molar refraction $(E)$, dipolarity/polarizability $(S)$, hydrogen bond acidity $(A)$ and basicity $(B)$, hexadecane-gas partition coefficient $(L)$ and $e, s, a, b, l$, and $c$ are the corresponding system parameters for each stationary phase listed in Table 2.2 and also in Table S2. Then, using the retention time information of an $n$-alkane series (Table S3) as well as the retention times of various other nonpolar chemicals from the training set (Table S2), we used least squares regression to determine the best fit coefficients of the following expressions:

$$
\begin{aligned}
& \log L_{1}=\alpha_{1} N_{i}^{*}+\alpha_{2} \\
& \log L_{2}=0.2613 N_{i}^{*}+\log \frac{T_{2, i}-\alpha_{3}}{T_{2, i}^{,}-\alpha_{3}}-0.557
\end{aligned}
$$

where $T_{2, i}$ is the second dimension retention time of the compound $i$, and $N^{*}$ and $T_{2, i}{ }^{*}$ are the carbon number and the second dimension retention time, respectively, of a hypothetical alkane eluting at the same first dimension retention time as the compound $i$. In fitting Equations 3.4 and 3.5, we employed $L_{1}$ and $L_{2}$ values calculated from the Abraham solute model for the first and second dimension stationary phases ${ }^{31}$ for the 56 compounds listed in Table S2. For the same compounds, the $N^{*}$ and $t_{2, i}{ }^{*}$ values were calculated from polynomial relationships of $N$ versus $T_{1}$, and of $T_{2}$ versus $N$, respectively. Both of these polynomial relationships were calculated from the retention times of the $n$-alkanes series (Tables S2 and S3). We note that Nabi et al. ${ }^{23}$ derived Equations 3.4 and 3.5 (including the known coefficients in Equations 3.5), for the same stationary phases as the ones in this study and using the $n$-alkanes as a reference series (the 
coefficients of Equations 3.5 would change for a different second dimension stationary phase or a different homologous series instead of the $n$-alkanes). Finally to evaluate $u_{1}$ and $u_{2}$ we used the following expressions:

$$
\begin{aligned}
& u_{1}=\log L_{1} \\
& u_{2}=\log L_{2}-\beta_{\text {orth }} \log L_{1}
\end{aligned}
$$

where $\beta_{\text {orth }}$ is a parameter that renders the $u_{1}$ and $u_{2}$ vectors orthogonal and was found to be 1.1315 by Nabi et al. ${ }^{23}$ The fitted values for $u_{1}$ and $u_{2}$ for the compounds used to derive the unknowns in Equations 3.4 and 3.5, are listed in Table S2.

\subsubsection{Nereis virens bioaccumulation experiments}

Bioaccumulation experiments with the polychaete Nereis virens were performed using four sediments contaminated with various HOCs. Sediments were collected with shovel or grab sampler from one location in Boston Harbor (Island End, Chelsea, MA, see map in Figure S1) in and 3 locations in San Francisco Bay, CA (C1800, C400 from Lauritzen Channel and 303.1 from outside the Lauritzen Channel, see map in Figure S2). Island End used to be the site of a coal gasification plan, and thus contains legacy coal tar pollution. Lauritzen Channel in Richmond Harbor is a Superfund site, which was contaminated with DDT and dieldrin due to on-shore pesticide packing and shipping activities until 1966. ${ }^{32}$ Despite remediation efforts between 1996 and 1999, the sediments in the channel remain contaminated with DDT and its degradation products DDE and DDD. Furthermore, recent investigations ${ }^{33}$ at the site showed that the sediments contain a large proportion of DDT compare to its degradation products, which was taken to be indicative of land-based sources from the embankments along the channel. C1800 C400 and 303.1 sediments were collected in March 2012 and used in a previous bioaccumulation study (Guilherme Lotufo), after which they were stored in freezer at $-18{ }^{\circ} \mathrm{C}$. C1800 sediment was collected from the northern part of the channel which experiences less flushing, and in consequence contained higher levels of DDXs than C400, which was collected from the middle of the channel (Figure S2). In addition to DDX contamination, previous analysis of the sediments of C1800 and C400 sediments showed high levels of PAHs, detectable levels of PCBs as well as a late eluting UCM trace. Lastly, 303.1 sediment collected from outside the DDT contaminated channel, is considered a reference site, containing background levels of PAHs, DDXs, and PCBs. 
The sediments were chosen due to their different HOC contaminations, as well as different geochemical properties (organic and black carbon contents).

The exposure protocol was adapted from standard methods for bioaccumulation tests. ${ }^{34}$ The Nereis virens ragworms were purchased from Aquatic Research Organisms (Hampton, NH) and were generally between 10 and $15 \mathrm{~cm}$, weighing 3-5 g (wet weight). Exposure tests were done in $1.5 \mathrm{~L}$ aquaria ( $10 \mathrm{~cm}$ diameter beakers), loaded with $\sim 800 \mathrm{~g}$ wet sediment (4 cm layer), 800 $\mathrm{mL}$ of artificial seawater and one ragworm. For each sediment, the experiment was conducted in triplicate, and the temperature was $21{ }^{\circ} \mathrm{C}$. The overlaying water was aerated with filtered house air, and changed every $3 \mathrm{~d}$ with fresh artificial seawater. ${ }^{35}$ After $28 \mathrm{~d}$, the worms were removed from the sediments and placed in $200 \mathrm{~mL}$ of clean seawater for depuration for $12 \mathrm{~h}$. No mortality of the worms was observed in any of the sediments and multiple burrows were noted at the end of the exposure in all aquaria (Figure S3). After depuration, the worms were frozen $\left(-20{ }^{\circ} \mathrm{C}\right)$ until extracted.

\subsubsection{Tissue extraction}

The worm tissue was thawed and ground with anhydrous $\mathrm{Na}_{2} \mathrm{SO}_{4}$ using a combusted mortar and pestle to the consistency of a free flowing powder and the procedure adapted from Kelly et al. ${ }^{36}$ was used to extract the contaminants as well as characterize the lipid content of the tissue. After addition of known amounts of recovery compounds (Table S4), the ground tissue was transferred along with the sodium sulfate to a pre-cleaned flask and extracted for 10 min with 30 $\mathrm{mL}$ of a 50:50 mixture of dichloromethane (DCM):hexane under sonication. The combined extract was concentrated to $1 \mathrm{~mL}$ using rotary evaporation. The lipid content of the extract was determined gravimetrically by measuring the residue left from $50 \mu \mathrm{L}$ of worm extract after solvent evaporation. The remaining extract was purified on silica gel columns. In short, $5 \mathrm{~g}$ of freshly prepared activated silica (100-200 mesh, EMD, purchased from Sigma Aldrich and combusted at $450{ }^{\circ} \mathrm{C}$ overnight) was deactivated with $250 \mu \mathrm{L}$ of MiliQ water (5\% deactivation) and loaded onto a $1 \mathrm{~cm}$ OD column fitted with a combusted glass wool plug. The column was topped with a $1 \mathrm{~cm}$ layer of anhydrous sodium sulfate and conditioned with hexane. The tissue extract (in hexane) was loaded and the column was eluted with $50 \mathrm{ml}$ hexane and $100 \mathrm{~mL}$ 95:5 hexane:DCM, which were collected together in a round bottom flask. The elution volumes were determined to be sufficient based on purification of extracts of blank worms spiked with a 
mixture of the 16 PAH target analytes (Table S5). The volume of the extract was reduced to 0.5 $\mathrm{mL}$ for PAH and DDT analysis, and $100 \mu \mathrm{L}$ for PCB analysis, and spiked with injection compounds prior to GC-MS analysis.

\subsubsection{Lipid analysis}

Although the DCM:hexane method ${ }^{36}$ described above presented the advantage that the lipid and contaminants could be extracted in one step, no comparison was found in the literature between lipid contents measured using this method and the more traditional Bligh and Dyer extraction. ${ }^{37}$ Furthermore, other studies compared the Bligh and Dyer method against other lipid extraction methods (Sohxlet extractions of freeze dried tissue with 10:7 acetone:hexane ${ }^{38}$ or acetone extraction of homogenized wet tissue ${ }^{39}$ ) and found that the lipid content results could vary by as much as factor of 3 . The difference was dependent on the type of tissue (e.g., less than $10 \%$ difference for fat fish, but factors of 2-3 more lipids were extracted with Bligh and Dyer versus Soxhlet extraction for lean fish, for polar and nonpolar lipids alike). In addition, compared to an acetone extraction at room temperature, the Bligh and Dyer protocol extracted a factor of 2 more lipids from mussel tissue, but a factor of 3 or more lipids from fish tissue. ${ }^{39}$ Thus, we compared the DCM/hexane procedure ${ }^{36}$ described in Section 3.2.5 against the Bligh and Dyer protocol $^{37}$ on several additional worms which were not exposed to the contaminated sediments. In the latter method, the thawed worm tissue was ground with a mortar and pestle, transferred to a centrifuge tube to which methanol and chloroform were added to achieve a ratio of 1:2:0.8 chloroform:methanol:water (assuming $\sim 80 \%$ water content for the worm tissue, which is appropriate given previous measurements of water content for Nereis virens ${ }^{40}$ ). The mixture was vortexed for $5 \mathrm{~min}$ and an equal volume of chloroform was added, followed by another $5 \mathrm{~min}$ of vortexing. Finally, a volume of water equal to the amount of water estimated for the original worm tissue was added and the mixture was then vortexed and centrifuged. The bottom chloroform layer was pipetted out and the last additions of chloroform and water were repeated twice more. The combined chloroform extract was then adjusted to $5 \mathrm{~mL}$ by evaporation, and the lipid concentration was determined gravimetrically. Both methods gave similar very results for the wet weight based lipid fractions $(1.59 \pm 0.25 \%$ for Bligh and Dyer versus $1.47 \pm 0.06 \%$ for DCM/hexane, Figure 3.2). Additionally, given that the Bligh and Dyer method is known to be very efficient at extracting both storage (nonpolar) and membrane (polar) lipids from tissues, the 
favorable comparison with the DCM:hexane method suggests that the DCM:hexane method of extraction is similarly efficient at extracting lipids, at least within the precision limit of our measurements and when applied to the Nereis virens tissue (which is reasonable since other abundant biomolecules like proteins or polysaccharides have low solubility in DCM and hexane $\left.{ }^{41}\right)$. Blank worms $(N=3)$ were saved from the batch of experimental worms used in bioaccumulation experiments, and had similar lipid content $(1.61 \pm 0.23 \%)$ to those used in the evaluating the two lipid measurements. Although no mortality was observed in any of the test sediments, the lipid content of the worms appeared to decrease after exposure to all sediments (Figure 3.2, average of $1.13 \pm 0.21 \%$ across all incubated worms). Since storage lipids are more labile than membrane lipids the decrease in lipid content most likely reflects a loss of storage lipids during incubation of the worms in the sediment.

\subsubsection{PE sediment equilibration.}

PE strips weighing $\sim 90 \mathrm{mg}$ each were placed in $500 \mathrm{~mL}$ round bottom flasks with $250 \mathrm{~g}$ wet sediment from each site and $300 \mathrm{~mL}$ MiliQ water. The flasks were then packed in a drum and mixed on a roller table at $30 \mathrm{rpm}$. After $28 \mathrm{~d}$, each PE strip was removed from the sediment slurry, rinsed with Mili Q water and divided into two pieces, one for GC-MS analysis (15 mg) and the rest for $\mathrm{GC} \times \mathrm{GC}$ analysis. The strip for GC-MS analysis was placed in DCM, spiked with recovery compounds (Table S4) and extracted three times with DCM. The combined extract was concentrated to an appropriate volume for analyte detection ( $1 \mathrm{~mL}$ for PAHs and DDXs, 100 $\mu \mathrm{L}$ for PCBs), spiked with injection compounds (Table S4) and analyzed by GC-MS. 


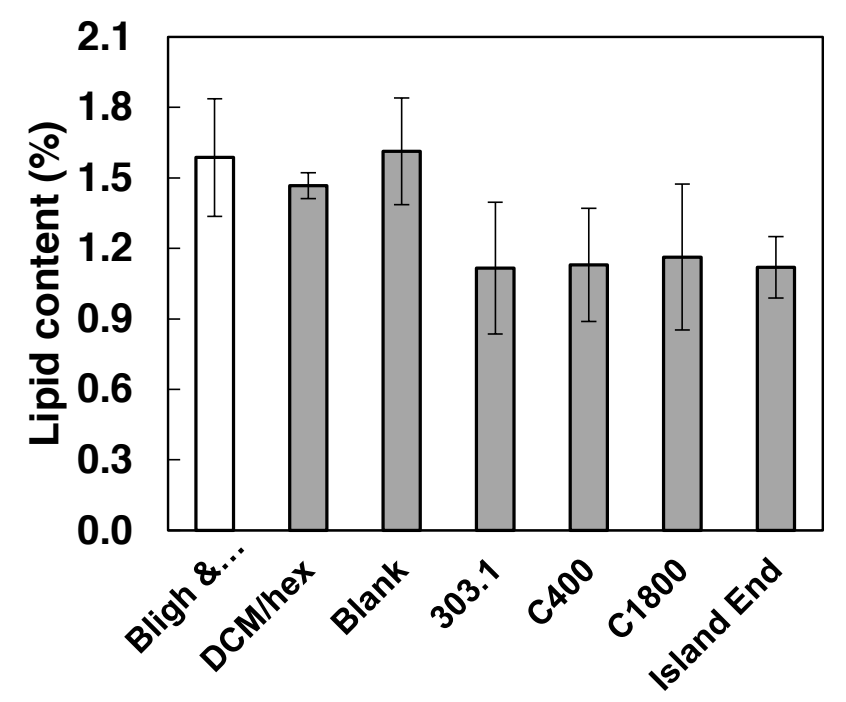

Figure 3.2 Lipid fractions measured on individual Nereis virens through the Bligh and Dyer method (empty bars) and DCM/hexane (filled bars). Also shown are the lipid contents measured for the unexposed worms (Blank) and worms exposed to each test sediment. All bars represent averages of three replicates and the error bars reflect one SD.

\subsubsection{Sediment characterization}

Prior to the bioaccumulation and PE equilibrations, aliquots of sediment from each site were oven dried at $60{ }^{\circ} \mathrm{C}$ overnight and used for chemical analysis, as well as measurements of water content, organic carbon $\left(f_{O C}\right)$ and black carbon $\left(f_{B C}\right.$, based on CTO-375 method $\left.^{27}\right)$ contents. Approximately $0.2 \mathrm{~g}$ of dry sediment were spiked with appropriate surrogate standards (Table S2) and extracted according to procedures similar to those in Chapter 6 . The determination of $f_{\mathrm{OC}}$ and $\mathrm{f}_{\mathrm{BC}}$ also followed the same procedures as in Chapter 6.

\subsubsection{GC-MS analysis}

PAH and PCB separations (see Figures S4 and S5 for chromatograms and analyte assignments) were performed on an Agilent J\&W DB-5 MS $60 \mathrm{~m}$ column $(0.25 \mu \mathrm{m}$ film thickness, $0.25 \mathrm{~mm}$ diameter, Agilent Technologies, Santa Clara, CA), at a helium flow of 2 $\mathrm{mL} / \mathrm{min}$. Injections $(1 \mu \mathrm{L})$ were done in pulsed pressure mode, with a splitless time of $1 \mathrm{~min}$. The inlet was held at $305{ }^{\circ} \mathrm{C}$. For PCB analysis, the oven was programed from 67 to $150{ }^{\circ} \mathrm{C}$ at 15 ${ }^{\circ} \mathrm{C} / \mathrm{min}$, then to $275{ }^{\circ} \mathrm{C}$ at $4{ }^{\circ} \mathrm{C} / \mathrm{min}$ and lastly to $292{ }^{\circ} \mathrm{C}$ at $2{ }^{\circ} \mathrm{C} / \mathrm{min}$. For PAH analysis, the oven 
temperature was initially at $67^{\circ} \mathrm{C}$, then programmed to $150{ }^{\circ} \mathrm{C}$ at $15^{\circ} \mathrm{C} / \mathrm{min}$, then to $275^{\circ} \mathrm{C}$ at 4 ${ }^{\circ} \mathrm{C} / \mathrm{min}$, and lastly to $300{ }^{\circ} \mathrm{C}$ at $2{ }^{\circ} \mathrm{C} / \mathrm{min}$, with a final hold time of $10 \mathrm{~min}$.

DDX analyses were performed on the same instrument, but equipped with an Agilent J\&W DB-XLB 30m column $(0.50 \mu \mathrm{m}$ film thickness, $0.32 \mathrm{~mm}$ ID, Agilent Technologies, Santa Clara, $\mathrm{CA}$ ), and using cold on-column injections (see Figure S6 for chromatograms and analyte assignments). The temperature program started at $50{ }^{\circ} \mathrm{C}$ and increased to $200{ }^{\circ} \mathrm{C}$ at $20{ }^{\circ} \mathrm{C} / \mathrm{min}$ and then to $274{ }^{\circ} \mathrm{C}$ at $4{ }^{\circ} \mathrm{C} / \mathrm{min}$ followed by a $15 \mathrm{~min}$ hold. The carrier gas was helium at 2 $\mathrm{mL} / \mathrm{min}$. The MS was run in selected ion monitoring mode.

Quantification of the compounds of interest (see Table S5 for all target analytes, their quantification and confirmation ions and retention times) was done based on response factors and the concentrations were corrected for recovery of the surrogate standards. Standards of the compounds of interest (PAH, PCB, DDXs) were run every 3-4 samples, to check the instrument stability and response factor consistency. For PAHs and DDXs, multiple concentration standards were analyzed (100-5000 and 25-2000 ng/mL, respectively), to match the concentration range of analytes in the samples. The standard deviations of response factors calculated from standards of different concentrations were typically $\sim 10 \%$ for DDXs, and $\sim 20 \%$ for PAHs. The average recoveries of surrogate standards were between $68-114 \%$ (Table S2). Detection limits for PAHs ranged from 5 to $20 \mathrm{ng} / \mathrm{g}$ and from $2-10 \mathrm{ng} / \mathrm{g}$ for PCBs in worms (lipid normalized), PE or sediment.

\subsubsection{GC $\times$ GC analysis}

$\mathrm{GC} \times \mathrm{GC}$ analyses was performed (1) on training set compounds (Table S1), to enable derivation of retention time or index based correlations for PE- and lipid-water partition coefficients, and (2) on select worm and PE samples suspected to contain UCM, with the ultimate goal of comparing accumulation of UCM in PE and worm tissue. The analysis was performed on an Agilent 7890 GC - FID, with a dual stage cryogenic modulator (Leco, Saint Joseph, MI). The injections were performed in splitless mode at $300{ }^{\circ} \mathrm{C}$ and the $\mathrm{H}_{2}$ carrier gas flow was maintained at $1 \mathrm{~mL} / \mathrm{min}$ throughout the run. The first dimension separation was performed on a Rxi-MS column (60 m, $0.25 \mathrm{~mm}$ ID and $0.25 \mu \mathrm{m}$ film thickness). The oven housing the first dimension column was held at $80{ }^{\circ} \mathrm{C}$ for $0.2 \mathrm{~min}$, then the temperature was 
ramped to $325{ }^{\circ} \mathrm{C}$ at $2{ }^{\circ} \mathrm{C} / \mathrm{min}$ with a final hold time of $0.5 \mathrm{~min}$. The flows exiting the first dimension column were modulated at $6 \mathrm{~s}$ modulation time and the compounds were transferred onto the second dimension BPX-50 column (1.5 m, $0.1 \mathrm{~mm}$ ID and $0.1 \mu \mathrm{m}$ film thickness). The temperature of the oven housing the second dimension column was maintained at a $5{ }^{\circ} \mathrm{C}$ offset with respect to the first dimension oven throughout the run. The modulator temperature was 15 ${ }^{\circ} \mathrm{C}$ higher than the temperature of the second dimension oven. The FID temperature was set at $330{ }^{\circ} \mathrm{C}$. To obtain more information about the identity of peaks which were not found in our standards (example in Figure S7), a subset of the samples was also analyzed on a second GC $\times$ GC system coupled with a time-of-flight detector (GC $\times$ GC-TOFMS, Leco, Saint Joseph, MI).

\subsection{Results and Discussion}

Previously (Chapter 2), we determined that the stationary phases used in this study, which are also commonly used for analysis of HOCs because of their stability at temperatures greater than $300{ }^{\circ} \mathrm{C}$, are not able to capture the electron-donation character of analytes. Thus, the $\mathrm{GC} \times \mathrm{GC}$ based method used in our work for determination of partition coefficients should not be applied to compounds that contain oxygen or nitrogen heteroatoms. Therefore, in this chapter, we will focus on HOCs containing only carbon hydrogen and chlorine atoms and their partitioning in $\mathrm{PE} /$ water/lipid systems.

\subsubsection{GC $\times$ GC based predictions for $K_{P E W}$.}

Starting with a training set made of 16 PAHs, 21 PCB, 12 HCs and 5 OCPs, with known $K_{P E W}$ values, we used a multivariable linear regression between $\log K_{P E W}$ and retention times, and found a relatively good correlation $\left(\mathrm{N}=54, \mathrm{R}^{2}=0.79, \mathrm{SE}=0.59\right.$, Figure 3.4). This $\log K_{P E W}$ versus retention time relationship would allow prediction of $K_{P E W}$ values with an error of about $0.59 \log$ units ( \pm factor of 4 ), which can be less than observed variability among different experimental determinations (Table S1). But closer inspection reveals that the correlation tends to predict higher than measured values for PAHs and HCs (largest differences of as much as 1 $\log$ unit for both HCs and PAHs with $K_{P E W}$ greater than $10^{6}$ ), but lower than measured for OCPs and PCBs (differences larger than $1 \log$ for OCPs, but less than $0.5 \log$ units for PCBs, Figure 3.3). Several studies have suggested that the partitioning of HOCs into polymers is driven by

their incompatibility with water. For example, Lohmann ${ }^{12}$ found that $K_{P E W}$ correlated with aqueous solubility for PCBs and PAHs $\left(R^{2}=0.94\right.$, $\left.\mathrm{SE}=0.27, N=65\right)$. In the study of Nabi et 
al., ${ }^{23}$ the authors found that using a retention index scale instead of retention time, and identical stationary phases to those used in this study, they were able to predict aqueous solubility with an average SE of $0.50 \mathrm{log}$ units, suggesting similar level of certainty as found for evaluating $K_{P E W}$.

Table 3.1 Regression lines for evaluating partition coefficients $K_{P E W}$ and $K_{P L W}$ from retention time and retention indices, as defined by Nabi et al. ${ }^{23}$

\begin{tabular}{|c|c|c|c|c|c|c|}
\hline $\begin{array}{c}\text { Property } \\
\text { (training set) }\end{array}$ & $\mathbf{E q} \#$ & Equation & SE & $N$ & $R^{2}$ & Range \\
\hline 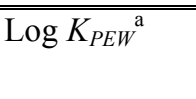 & 3.8 & 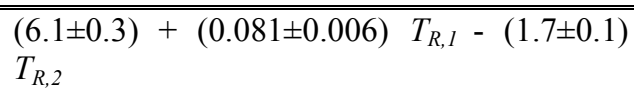 & 0.59 & 54 & 0.79 & $\log K_{P E W} 3.0-8.4$ \\
\hline $\log K_{P E W}{ }^{\mathrm{a}}$ & 3.9 & $(-2.2 \pm 0.4)+(2.0 \pm 0.1) u_{1}-(7.7 \pm 0.5) u_{2}$ & 0.44 & 54 & 0.88 & $\log K_{P E W} 3.0-8.4$ \\
\hline $\log K_{P E W}^{\mathrm{b}}$ & 3.10 & $(-2.2 \pm 0.3)+(2.0 \pm 0.1) u_{1}-(7.7 \pm 0.4) u_{2}$ & 0.35 & 49 & 0.93 & $\log K_{P E W} 3.0-8.4$ \\
\hline $\log K_{P L W}{ }^{\mathrm{c}}$ & 3.11 & $(-1.0 \pm 0.4)+(1.8 \pm 0.1) u_{1}-(5.6 \pm 0.7) u_{2}$ & 0.34 & 30 & 0.95 & $\log K_{P L W} 3.4-8.0$ \\
\hline
\end{tabular}

\footnotetext{
${ }^{\mathrm{a}}$ Training set in Table S1

${ }^{\mathrm{b}}$ Training set in Table S1, excluding OCPs

${ }^{\mathrm{c}}$ Training set in Table 2.1, excluding $p$-xylene and chlorobenzene which eluted outside the acquisition window used in this study
}

Employing the retention index method, we first calculated the retention indices $u_{1}$ and $u_{2}$ (Table S1), and performing the regression for the same training set against retention indices instead of retention times, we obtain a better relationship for $K_{P E W}\left(R^{2}=0.88, \mathrm{SE}=0.44( \pm\right.$ factor of 3), Table 3.1). Comparing the estimations against the experimental values, we no longer observe a HOC-family dependent trend (Figure 3.3). However for most of the OCPs (specifically 4,4'-DDD, 4,4'-DDT, heptachlor and aldrin), the GC $\times$ GC-based predictions of $K_{P E W}$ are larger than the experimental $K_{P E W}$ value by $1 \log$ unit or more (Table S1). We are fairly confident that these deviations are not due to experimental uncertainty, because independent measurements done in our lab for $K_{P E W}$ of 4,4'-DDD, 4,4'-DDE and 4,4'-DDT (Chapter 4) agreed well with previously published values. ${ }^{28}$ Other authors have also found large deviations between experimental values and $K_{O W}$ based LFER predictions of $K_{P E W}$ for OCPs (Hale et al. ${ }^{28} \mathrm{R}^{2}=0.53$ ), whereas similar relationships for PAHs and PCBs tend to have $\mathrm{R}^{2}>0.9 .{ }^{12}$ One explanation could be that partitioning into polymers is influenced by the geometry of the molecule, as the 
relationships derived from $\mathrm{GC} \times \mathrm{GC}$ appear to capture well planar molecules like PAHs and some PCBs, but predict smaller than observed values for the OCPs as well as PCBs with four chlorine atoms in the ortho position ( $\Delta_{\text {predicted-measured }}$ for PCB $155-0.7$ compared to -0.3 to 0 for other PCBs, Table S1). However, similar deviations for OCPs are observed if, instead of $K_{P E W}$, we attempt to derive $K_{O W}$ versus retention time relationships, and partitioning into $n$-octanol is less likely to be influenced by geometry of the molecule. Similarly, other GC $\times$ GC-based attempts to characterize the properties of OCPs have also found as much as 1 log unit difference between measured and predicted organic carbon-water partition coefficients for 4,4'-DDT and aldrin. ${ }^{23}$ In addition, there are other molecules that are not planar such as the LABs and ACHs to which we have applied the $\mathrm{GC} \times \mathrm{GC}$-based relationship for evaluating their $K_{P E W}$ values, and the observed deviations between measured and estimated were not as large as those for OCPs ( $\Delta_{\text {predicted-measured }} \sim 0.5$ both positive and negative, Figure 3.3). Thus, although it does not appear that the geometry of the molecule explains the observed deviations for OCPs, it is apparent that these molecules behave differently than the PAHs or PCBs and further investigations are needed to understand the best way to model the properties of OCPs.

Nonetheless, it is important to note that eliminating the OCPs from the training set does not significantly change the regression line, but better constrains the regression coefficients (Table 3.1). Both $K_{P E W}$ relationships (RT-based Equation 3.8 and RI-based Equation 3.9) show a positive correlation of $K_{P E W}$ with the first dimension retention time. This makes sense, as we would expect $K_{P E W}$ to increase with molecular size. $K_{P E W}$ decreases, however, with retention in the second dimension, which is also consistent with the physical understanding that, as polarity increases, compounds of similar size are more soluble in water, leading to a decrease in $K_{P E W}$. We note that the intercept is negative in the case of RI regressions, which is related to the way $u_{2}$ is defined (Equation 3.7). This definition allows it to have both positive and negative values (range of -0.3 to +0.3 for the training set compounds in Table S1). 

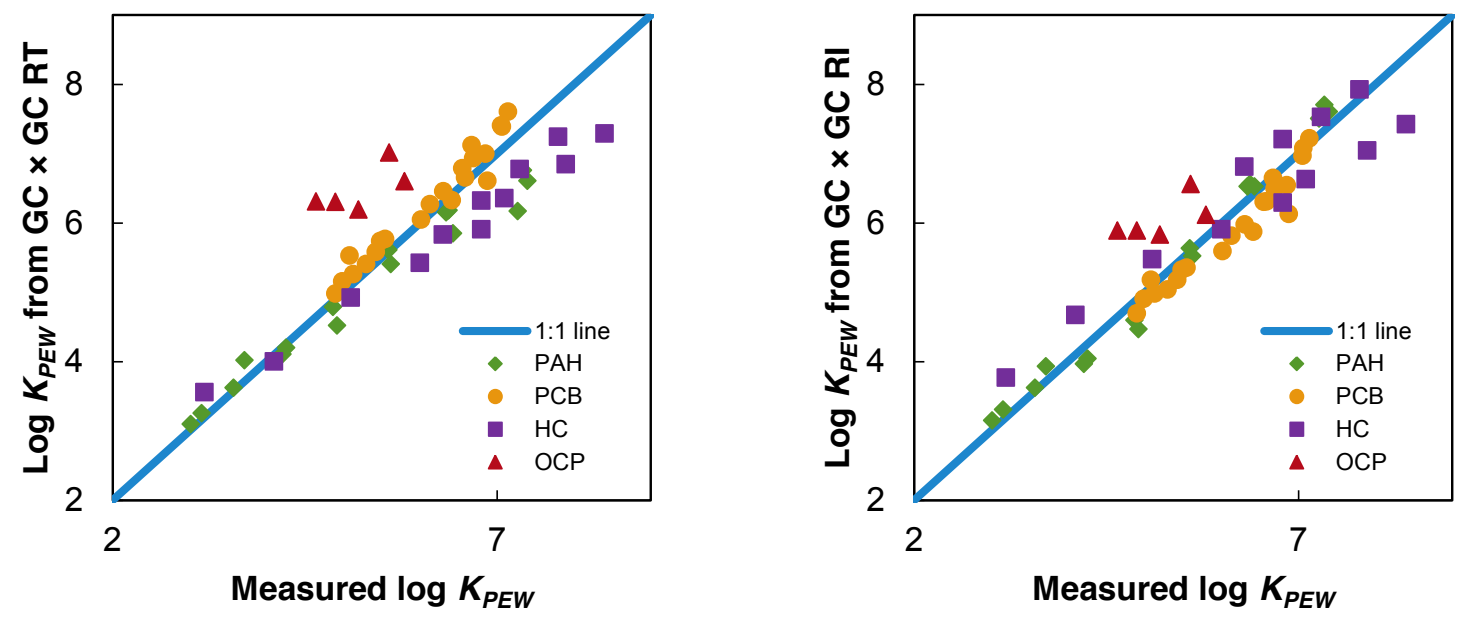

Figure 3.3 Comparison between measured and $\mathrm{GC} \times \mathrm{GC}$-derived $K_{P E W}$ values using retention times (left) and retention indices (right) for PAHs, PCBs, hydrocarbons (HCs) and organochlorine pesticides (OCPs) in Table S1 training set.

Early efforts of using chromatography to estimate partition coefficients employed a single column. But such an approach would not work for the diversity of compounds used in this study, even though they are mostly nonpolar chemicals (limited hydrogen bonding character as defined by Abraham $A$ and $B$ parameters being $<0.06$ and 0.5 , respectively). By evaluating the dependency of $K_{P E W}$ on the first dimension retention index, we find that each HOC family (PCB, PAHs, HCs) has a distinct slope (Figure 3.4), similarly to the findings of Arey et al. for other physico-chemical properties such as $K_{O W}$ or aqueous solubility. ${ }^{17}$ Regressing $\log K_{P E W}$ solely against the first dimension retention index $\left(u_{1}\right)$ for all compounds, we see that the first dimension explains only $29 \%$ of the variance in the $K_{P E W}$ training set, whereas fitting against both dimensions explains an additional 57\%. In contrast, using raw retention times, the first dimension retention time can explain $26 \%$ of the variance, with an additional $49 \%$ of the variance captured by the addition of the second dimension. Thus, by using the retention indices approach, which adjusts the retention time in the second dimension to a hypothetical value at 120 ${ }^{\circ} \mathrm{C}$ (i.e., the temperature at which the ASM expressions for $L_{1}$ and $L_{2}$ were derived ${ }^{31}$, also in Table 2.2), we seem to better incorporate the information captured by elution of compounds on the second dimension. Lastly, we note that fitting $K_{P E W}$ against either $T_{2}$ or the second dimension vector $u_{2}$ leads to very low $R^{2}$ values ( 0.053 in both cases), which make sense since neither $T_{2}$ nor $u_{2}$ contain information about the size of the molecules. Fitting against $L_{2}$, which is 
representative of using the second dimension column as a first dimension column captures $46 \%$ of the variability in the training set data, more than when the fit was performed against $L_{1}$, emphasizing the contribution of polarity in PE-water partitioning.

\subsubsection{GC $\times$ GC based predictions for $K_{P L W}$.}

In order to use $\mathrm{GC} \times \mathrm{GC}$ retention information to evaluate the bioaccumulation potential of HOCs from their corresponding accumulation in passive samplers, a second relationship is needed that relates the lipid-water partition coefficient, $K_{P L W}$, to the $\mathrm{GC} \times \mathrm{GC}$ retention indices and which can be used in combination to the one we constructed for $K_{P E W}$ in the previous section. Developing the $K_{P L W}$ versus $\mathrm{GC} \times \mathrm{GC}$ retention times relationship was the focus of Chapter 2, and we revisit it here briefly, by considering additional training set compounds (aliphatic and monoaromatic hydrocarbons) and the implications of employing retention indices instead of retention times.

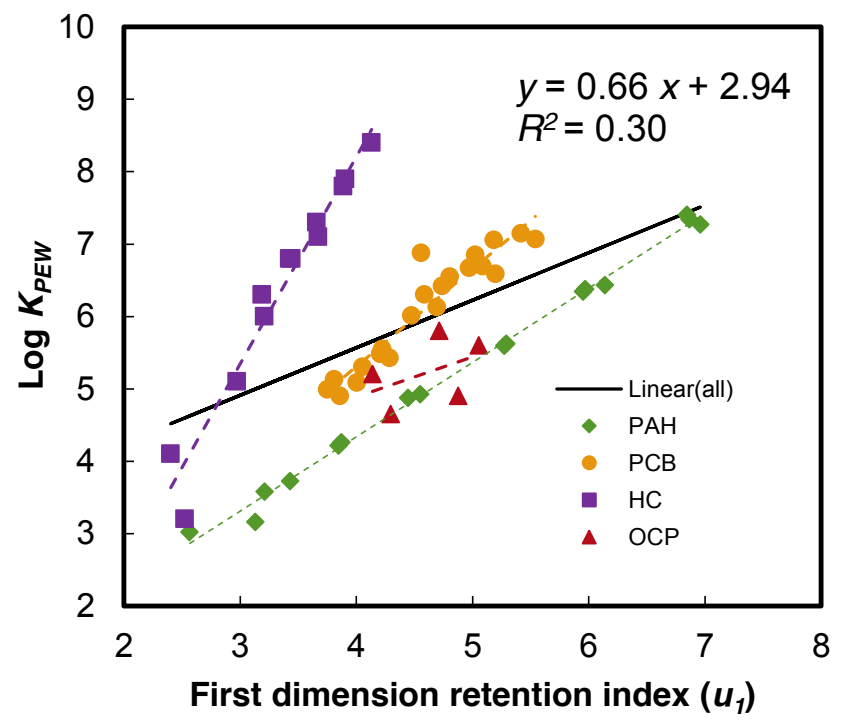

Figure 3.4 Correlation of $\log K_{P E W}$ for training set compounds (Table S1) versus first dimension retention vector, $u_{l}$. Black solid line and equation represents the linear regression through all points, whereas dotted line represents the linear fit for each compound class.

For $K_{P L W}$, the training set comprised mostly of compounds previously used to train $K_{P L W}$ versus retention time relationships (Chapter 2, Table 2.1), with the addition of a few hydrocarbons. As no $K_{P L W}$ values could be found in the literature for hydrocarbon compounds 
with $N^{*}>10\left(N^{*}\right.$ hypothetical carbon number, defined in Section 3.2.3), we calculated these values using the polyparameter relationship of Endo et al. ${ }^{42}$ (also reproduced in Table 2.2). We note that the training set used for derivation of polyparameter relationship of Endo et al. ${ }^{42}$ did also include saturated hydrocarbons, but they were typically smaller than $n$-octane $\left(N^{*}<8\right)$. The addition of hydrocarbons to the training set was important to ensure similar distribution of training set compounds across the two dimensions as in the case of $K_{P E W}$ (Section 3.3.1).

Using a two dimensional regression of $K_{P L W}$ values against the retention indices $u_{1}$ and $u_{2}$, calculated using the same equations as when developing the $K_{P E W}$ relationships, we obtain a good fit with an $\mathrm{R}^{2}$ of 0.94 and $\mathrm{SE}$ of $0.34(\mathrm{~N}=30)$. The fit is comparable, but not better than the equivalent relationship calculated based on retention time instead of indices $\left(R^{2} 0.97, S E=0.24\right.$, $N=30$ ). This is the opposite trend compared to the regression lines derived in the case of $K_{P E W}$, when the statics for RI-based regression were significantly better than RT-based one (Table 3.1, compare Equations 3.8 and 3.9). Overall, the difference between the two prediction methods for $K_{P L W}$ is quite small, and could reflect noise in the training set data (e.g., lack of experimental values for hydrocarbons).

It is interesting that the retention time approach works at all, since compared to retention indices, retention times are not a true thermodynamic scale. ${ }^{23}$ We observe that the contour lines of $\log K_{P L W}$ drawn across the $\mathrm{GC} \times \mathrm{GC}$ space using both retention times and retention indices (Figure 3.5), can be approximated by straight lines particularly above second dimension retention times of $3 \mathrm{~s}$, which is the region where the more polar molecules elute (OCPs, PAHs, and PCBs as opposed to $n$-alkanes and other saturated molecules that elute in the region of $2-3 \mathrm{~s}$ ). Thus employing either RI or RT based regressions for these types of HOCs leads to comparable results. However, in the region between 2 and $3 \mathrm{~s}$ (where aliphatic or monoaromatic series like $n$ alkanes or alkyl benzenes elute), the predictions differ significantly between the two models. This region is scarce in experimental data for $K_{P L W}$, but better constrained for $K_{P E W}$. The $K_{P E W}$ values for $n \mathrm{ACHs}$ and LABs in the region of low polarity compounds are better characterized when using retention indices compared to retention times (SE for $12 \mathrm{HCs}$ reduced from 0.7 to 0.5 log units by using RI instead of RT-based correlations. 

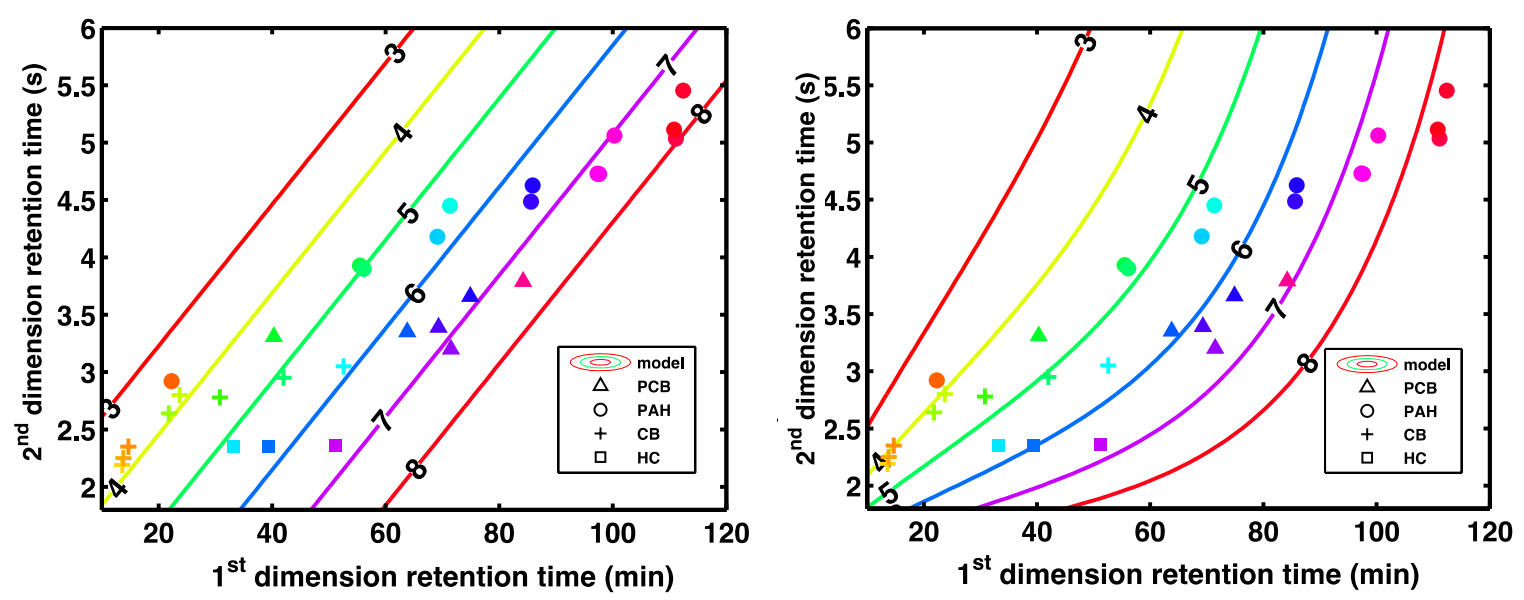

Figure 3.5 Comparison between retention time (left) and retention indices (right) methods for evaluating $\log K_{P L W}$ across the $\mathrm{GC} \times \mathrm{GC}$ retention space. The training set compounds are plotted at their corresponding retention times and the color of the point is reflective of the experimental $\log K_{P L W}$ value of each compound. The model, drawn as contours of $\log K_{P L W}$, is able to correctly predict the $\log K_{P L W}$ values when compounds of the same color overlap with the same color contour line.

\subsubsection{GC $\times$ GC-based predictions of lipid/PE differential accumulation of HOCs}

Combining the relationships for evaluating $K_{P E W}$ and $K_{P L W}$ from $\mathrm{GC} \times \mathrm{GC}$ retention time and retention indices, we find that, at equilibrium, the differences between accumulation in lipids and $\mathrm{PE}$ are small (at most one order of magnitude), but different HOC classes will prefer either the lipid or the PE phase (Figure 3.6). The preference for the lipid or PE phases is mainly driven by polarity, as evidenced by an increase in the $K_{P L-P E}\left(=K_{P L W} / K_{P E W}\right)$ values with increase in the second dimension time. We note that polarity is often an operational definition, as it could refer to the order that compounds elute from a silica column, the value of the compounds' $S$ parameter, or the elution order of compounds on a polar stationary phase such as the one used herein as second dimension GC $\times$ GC column. Throughout this manuscript, we will refer to the polarity of contaminants mainly in terms of their elution on the $2^{\text {nd }}$ dimension $\mathrm{GC} \times \mathrm{GC}$ stationary phase, on which $n$-alkanes are retained the least (least polar) and PAHs are the most retained (most polar) out of the HOCs investigated in this study (i.e., we do not exclude that there may be compounds that are retained more that the PAHs on the $2^{\text {nd }}$ dimension $50 \%$ phenyl polysilphenylene-siloxane column). According to the model results, the nonpolar $n$-alkanes favor the PE phase ( $K_{P L-P E}$ of $\left.0.2-0.3\right)$, whereas PAHs favor the lipid phase $\left(K_{P L-P E}\right.$ of $\left.\sim 6\right)$. PCBs are 
less polar than PAHs, but significantly more polar than $n$-alkanes, and the model estimates that PCBs will also favor partitioning into the lipids $\left(K_{P L-P E}\right.$ of 2-3). Compound size has a secondary effect on the partitioning, with the tendency to partition in the lipids decreasing for larger compounds, although overall this effect is very small (e.g., range of $K_{P L-P E}$ varies by only a factor of 3 in the first dimension as compared to a factor of 100 in the second dimension). The predictions of lipid-PE partitioning are similar whether we use the RI or RT based correlations (Table 3.1).
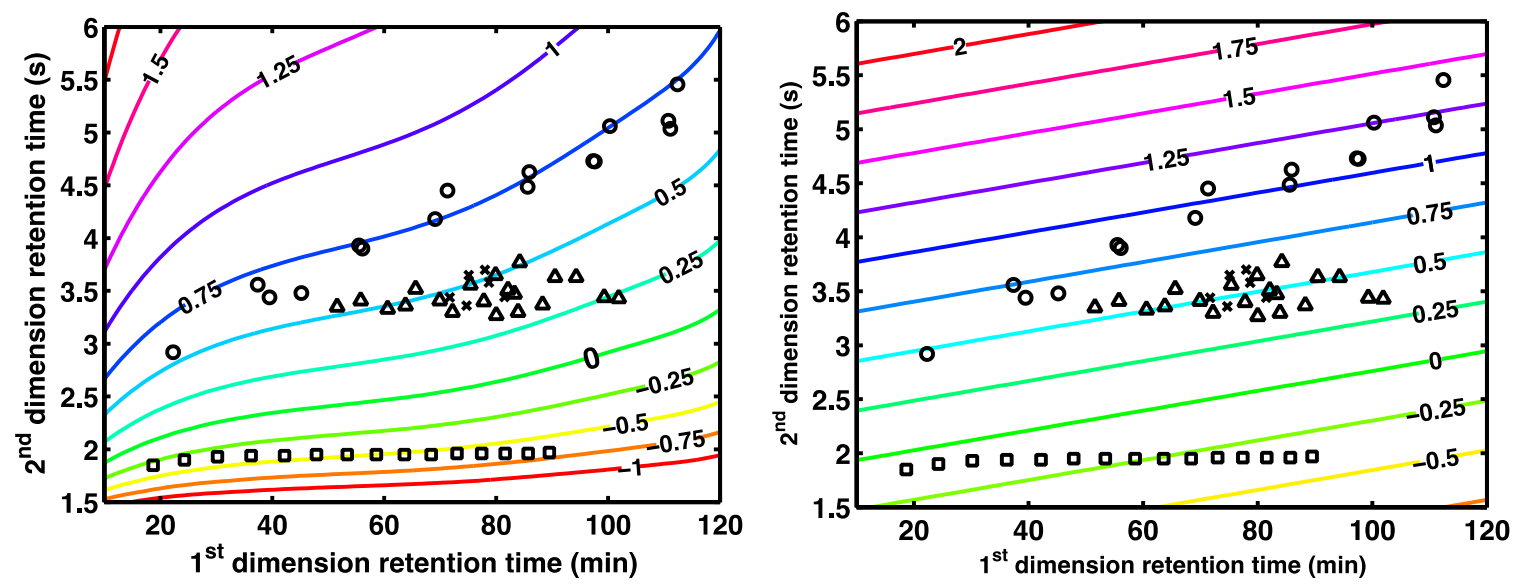

Figure 3.6 Contour plots of the ratio of $K_{P L W} / K_{P E W}$, in $\log _{10}$ space, as derived from correlations (Table 3.1) against retention indices (left) and retention times (right). The elution times of various HOCs discussed in the text are overlaid on the contours: PAHs (circles), PCBs (triangles), OCPs (crosses) and the $n$-alkane series with 11-25 carbon atoms (squares).

The importance of polarity for partitioning between lipids and PE can also be understood in the context of two well known solvation models: the Abraham solvation model (ASM, introduced in 3.2.3) and the Scatchard-Hildebrand solvation theory. Although no ASM ppLFERs have been developed for PE, Sprunger et al. ${ }^{43}$ found that the partitioning between PDMS and water $\left(K_{P D M S / W}\right)$ can be described by the following equation with the error for each coefficient in parentheses:

$$
\begin{gathered}
\log K_{P D M S / W}=0.246(0.072)+0.568(0.053) \mathbf{E}-1.305(0.088) \mathbf{S}-2.565(0.106) \mathbf{A}-3.928 \\
(0.119) \mathbf{B}+3.573(0.059) \mathbf{V}
\end{gathered}
$$


Comparing this relationship with existing ones for $K_{P L W}{ }^{42}$ (Table 2.2), we find that while both partition coefficients have about the same system constant and dependency on molecular volume $(V)$, they are different in dependency on $E S A$ and $B$ (defined in section 3.2.3). The ratio between lipid and PDMS partitioning calculated using ASM $\left(K_{P L W} / K_{P D M S-w a t e r} 5-10\right.$ for PCBs, and 5-30 for PAHs) is consistent with that deduced for lipid-PE partitioning from $\mathrm{GC} \times \mathrm{GC}$ retention, remembering that PDMS-water partition coefficients are usually 3-10 fold smaller than corresponding PE-water partition coefficients. ${ }^{15}$ The difference between PCBs and PAHs is due to the PAHs having both larger excess molar refraction $(E)$ and basicity $(B)$.

Secondly, in the Hildebrand-Scatchard theory (Poerschmann et al. ${ }^{44}$ and references within), the change in energy on mixing two substances (which dictates the compatibility of the two substances), is proportional to the difference in their solubility parameters $(\delta)$. Polyethylene ${ }^{45}$ and lipids ${ }^{44}$ have different solubility parameters $\left(\delta_{\mathrm{PE}}=16.3-17.9 \mathrm{MPa}^{1 / 2}, \delta_{\text {lipids }} 20.7-21.8\right.$ $\mathrm{MPa}^{1 / 2}$ for fish adipose tissue and DMPC vesicles). In consequence, chemicals ${ }^{44}$ like $n$-alkanes $\left(\delta_{\text {n-alkane }}=15.3-16.0 \mathrm{MPa}^{1 / 2}\right)$ will be more soluble in PE than in lipids, whereas PCBs $\left(\delta_{\mathrm{PCBs}} 18.9\right.$ - $\left.19.9 \mathrm{MPa}^{1 / 2}\right)$ and PAHs $\left(\delta_{\mathrm{PAHs}} 19.9\right.$ - $\left.20.6 \mathrm{MPa}^{1 / 2}\right)$ will prefer lipids over PE, supporting the results of our GC $\times$ GC model.

We also checked the performance of the $\mathrm{GC} \times \mathrm{GC}$ correlations by comparing the estimated $K_{P L-P E}$ for several HOCs based on their retention time, against independent estimates based on available literature values for $K_{P L W}$ and $K_{P E W}$. In general, we found good agreement for the $\log$ $\mathrm{K}_{\mathrm{PL}-\mathrm{PE}}$ of PAHs (within $0.2 \log$ units) and PCBs (0.1 log units), but not as good for DDXs (0.5-1 $\log$ units for DDT and DDD). The independent estimates for $K_{P L-P E}$ of PAHs were calculated based on experimental values for both $K_{P E W}$ (Table S1) and $K_{P L W}$ (Chapter 2, Table 2.1). For PCBs and DDXs, the $K_{P L-P E}$ values were based on experimental $K_{P E W}$ values, ${ }^{14,25,28}$ but experimental $K_{P L W}$ values were not available, and were instead calculated from a $K_{O W}$ LFER $(\log$ $K_{P L W}=1.01 \log K_{O W}+0.12$, Endo et al. ${ }^{42}$, with $K_{O W}$ values from Hawker and Connell ${ }^{46}$ and $\mathrm{ASTDR}^{47}$ ). Gschwend et al. ${ }^{48}$ also evaluated the lipid-PE partitioning of PCBs and found that PCBs favor lipids over PE by a factor of $1.6 \pm 0.3$, which is lower than the GC $\times$ GC-based estimation ( $K_{P L-P E}$ of 2-3) but only by 0.1 to $0.3 \mathrm{log}$ units. The lack of experimental $K_{P L W}$ values could be the reason for the poor agreement between $\mathrm{GC} \times \mathrm{GC}$ and other estimates of $K_{P L-P E}$ of 
DDXs, although it should also be noted that GC $\times$ GC-based regressions did not properly characterize $K_{P E W}$ for these compounds either.

Lastly, as mentioned before, the partitioning into lipid tissue could involve both partitioning into storage lipids such as triglycerides (TG), as well as into membrane phospholipids (PL). However, for PCBs and PAHs, previous studies found little difference between partitioning into

TG or PL phases (at most 0.2 log units), ${ }^{42}$ suggesting that even if the distribution of lipids would be skewed towards TGs, estimations of bioaccumulation would remain the same. Out of all the compounds considered in this study, notable differences between partitioning into TGs and PLs would be significant only for the more saturated portion of the UCM. For example, using ASM models $^{42}$ for TG and PL (equations also reproduced in Table 2.2) we calculate that $n \mathrm{C} 8$-benzene $(E=0.579 S=0.48 A=0 B=0.15 \mathrm{~V}=2.13)$ would preferentially accumulate into $\mathrm{TG}\left(\mathrm{K}_{\text {olive-oil/water }}=\right.$ $\left.10^{7}\right)$ over PL $\left(K_{P L W}=10^{6}\right.$ - both measured and calculated with ASM equation in Table 2.2), by a factor of 10 However, given that proportion of storage lipids in mature polychaetes are usually smaller than membrane lipids, ${ }^{22}$ the overall effect on the bioaccumulation would be small (overall lipid-water partition coefficient would be $\sim 0.5 \mathrm{log}$ units larger than the $\mathrm{GC} \times \mathrm{GC}$ estimated $K_{P L W}$, assuming maximum TGs are $30 \%$ of the total lipids).

\subsubsection{Accumulation of HOCs in Nereis virens and GC $\times$ GC based predictions}

To test whether the $\mathrm{GC} \times \mathrm{GC}$ retention information could be used to estimate the accumulation in biota of various HOCs based on corresponding accumulation in PE, we compared the results of the previous section against experimental measurements of HOC in PE and in the Nereis virens tissue. Firstly, using the GC-MS results, we compared the measured $\mathrm{C}_{\text {lipid }} / \mathrm{C}_{\mathrm{PE}}$ ratio for PCBs, PAHs, and DDXs (measured concentrations of all target analytes in Tables S6-S10), against GC $\times$ GC based predictions of the lipid-PE partition coefficient for each chemical. These two quantities should be equal if both the PE and the worm tissue are in chemical equilibrium with the sediment. Secondly, we used GC $\times$ GC - FID analysis of PE and extracts of worms incubated with C1800 and Island End sediments, to compare the measured accumulation of UCM compounds in lipids versus the accumulation estimated from PE and $K_{P L}$ $P E$ Values calculated using Equations 3.10 and 3.11. 
PCBs. The concentrations of PCB congeners in worms exposed to the test sediments were factors of 2 - 250 higher than the concentrations in the blank worms (Figure 3.7), suggesting the measured PCBs were due to uptake from contaminated sediment. Out of the 20 NOAA congeners quantified, five congeners (\#8, \#126, \#195, \#206 and \#209) were below detection in most samples, and were not considered in following discussions. Individual PCB congener concentrations in the blank worms were typically less than $20 \mathrm{ng} / \mathrm{g}_{\text {lipid }}$, with the exception of PCBs 153 and 138 (70 and $30 \mathrm{ng} / \mathrm{g}_{\text {lipid }}$, respectively). The sum of the quantified PCB congeners (Table 3.2) was lowest for the worms incubated in 303.1, the reference sediment $\left(1.1 \mu \mathrm{g} / \mathrm{g}_{\text {lipid }}\right)$, followed by C400 $\left(1.4 \mu \mathrm{g} / \mathrm{g}_{\text {lipid }}\right)$, and highest in C1800 $\left(5.8 \mu \mathrm{g} / \mathrm{g}_{\text {lipid }}\right)$. The total PCBs were not directly quantified, but based on previous research, ${ }^{49}$ we estimate that they would be two fold higher than the sum of NOAA 20 PCBs (e.g., $\sim 12 \mu \mathrm{g} / \mathrm{g}_{\text {lipid }}$ for C1800 worms). Tri- and tetrachlorinated congeners dominated the PCBs accumulation in C1800 (29 and 35\% of total), whereas PCBs measured in C400 worms were more evenly distributed across tetra-, penta-, hexachlorinated congeners (22, 30 and 29\%). The distribution of PCBs was generally consistent with Aroclors 1248, 1254 and 1260, which have been previously measured in sediments and biota at the sediment collection site. ${ }^{50}$ PCB concentrations measured in worms incubated with Island End sediment (1.5 ug/g $\mathrm{g}_{\text {lipid }}$, Table 3.2) were similar to those for C400, but the distribution was skewed in the case of Island End toward hexa- and heptachlorinated congeners $(66 \%$ of total).

Accumulation of PCBs in PE tumbled with sediment was similar to the accumulation observed in the worms both in terms of quantity and distribution of congeners. Island End and C400 PE showed similar amounts of total PCBs (1.8 and $1.4 \mathrm{ug} / \mathrm{g}_{\mathrm{PE}}$, Table 3.2), and as in the case of the worm tissue analysis, the hexa- and heptachlorinated congeners in Island End PE accounted for $69 \%$ of the total PCB concentration. The PE incubated with C1800 sediment showed the highest concentration of total PCBs (sum of the 20 measured congeners, $3.6 \mu \mathrm{g} / \mathrm{g}_{\mathrm{PE}}$ ), but tri and tetrachlorinated congeners made up only $47 \%$ of total concentration compared to $64 \%$ in the worm tissue. 


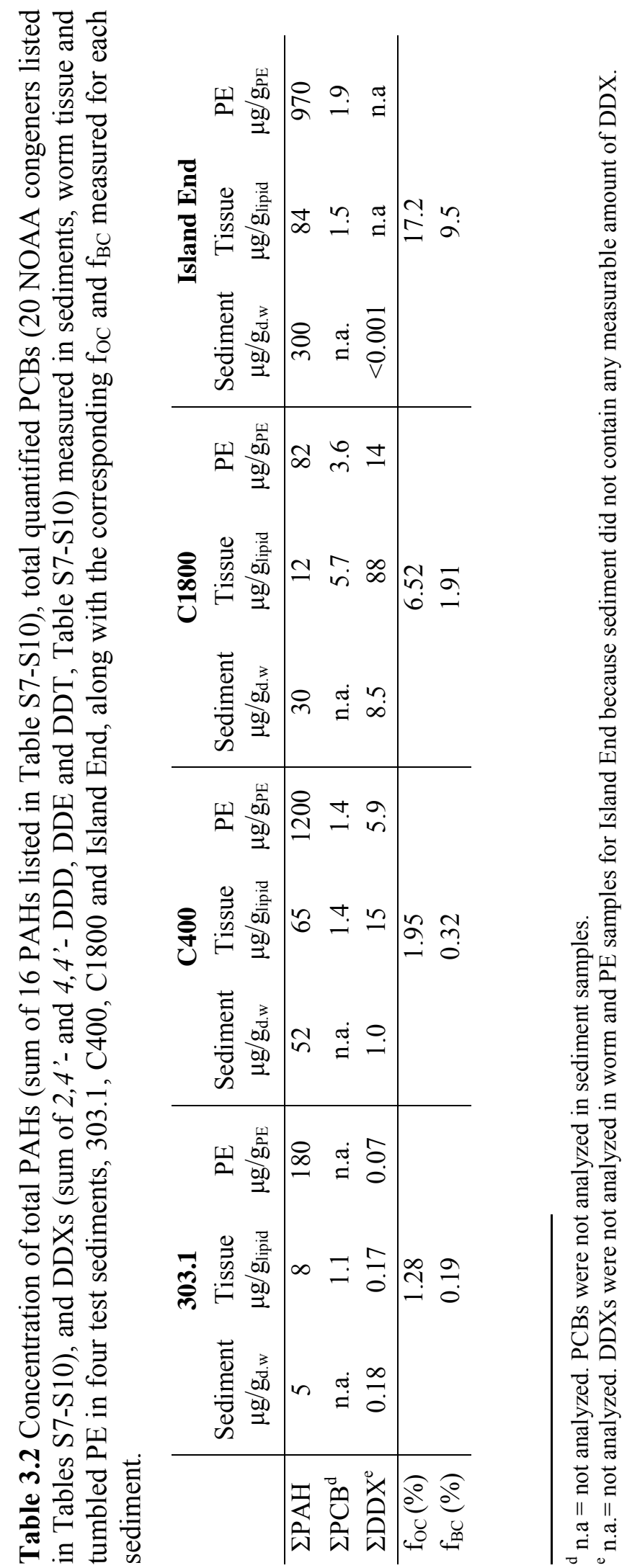



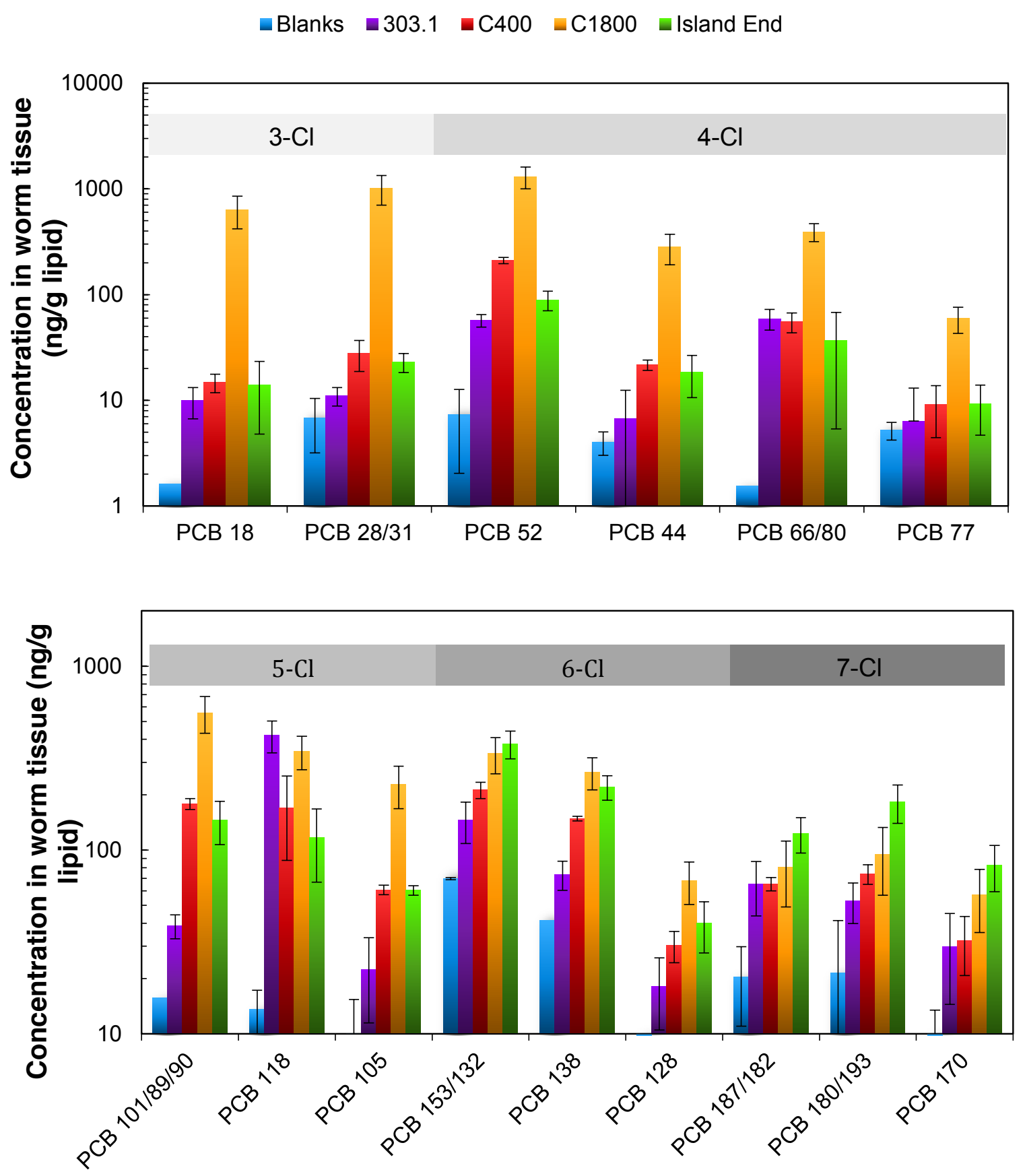

Figure 3.7 Lipid-normalized PCB concentrations measured in unexposed worms (blanks) and in worms exposed to the four test sediments. Concentrations in all test worms were at least a factor of 2 higher than in the blank worms (with exception of PCB 77). Worms exposed to C1800 sediment showed a large proportion of tri- and tetrachlorobiphenyls $(64 \%$ of total concentration of 20 congeners measured), whereas worms incubated in Island End sediment showed a distribution of PCBs skewed towards the hexa- and hepta-chlorobiphenyls $(66 \%$ of total concentration of the 20 congeners measured) 
Concentrations of individual congeners in the PE were linearly correlated with the corresponding lipid-normalized concentrations in worms (Figure 3.8 top). The slopes of the linear fits were close to 1 for $\mathrm{C} 400\left(\mathrm{R}^{2}=0.91, \mathrm{C}_{\mathrm{PE}}=1.02 \pm 0.09 \mathrm{C}_{\text {lipid }}\right)$ and Island End $\left(\mathrm{R}^{2}=0.90\right.$, $\left.\mathrm{C}_{\mathrm{PE}}=1.14 \pm 0.10 \mathrm{C}_{\text {lipid }}\right)$ sediments, and 0.5 for $\mathrm{C} 1800\left(\mathrm{R}^{2}=0.80, \mathrm{C}_{\mathrm{PE}}=0.49 \pm 0.07 \mathrm{C}_{\text {lipid }}\right)$. Using the $\mathrm{GC} \times \mathrm{GC}$ derived $K_{P L-P E}$ values for PCBs (ranging from 2.5 - 4, Figure 3.6), we calculated the expected concentration in the lipids based on the measured $\mathrm{C}_{\mathrm{PE}}$ 's. The predicted and measured PCB concentrations in lipids were in good agreement for $\mathrm{C} 1800$ sediment $\left(\mathrm{C}_{\text {lipid,predicted }}\right.$ $\left.=1.7 \pm 0.2 \mathrm{C}_{\text {lipid,measured, }} \mathrm{R}^{2}=0.83\right)$, but deviated by a factor of 3 for $\mathrm{C} 400\left(\mathrm{C}_{\text {lipid,predicted }}=3.1 \pm\right.$ $\left.0.2 C_{\text {lipid,measured }}, R^{2}=0.92\right)$ and Island End $\left(C_{\text {lipid,predicted }}=3.14 \pm 0.24 C_{\text {lipid,measured, }}, R^{2}=0.93\right)$ sediments (Figure 3.8 bottom).

Thus, passive sampler concentrations appear to overestimate the measured worm concentrations by factors of 2-3, suggesting that the worms may have not reached thermodynamic equilibrium with the sediments during the $28 \mathrm{~d}$ exposure. However, Bennett et al. ${ }^{51}$ investigated the kinetics of uptake and release of PCB accumulation by Nereis virens, and found that whole body elimination rates (1) ranged from 0.06 to $0.1 \mathrm{~d}^{-1},(2)$ decreased weakly with $K_{O W}$, and (3) were relatively constant across three different sediments tested. These elimination rates suggest that $28 \mathrm{~d}$ should have been enough time for the PCBs to reach at least 90\% steady state concentration in the worm tissue. In fact, for both C400 and Island End sediments, we observe no dependency of the predicted/measured ratio on congeners' $K_{O W}$, and only a slight correlation for C1800 test sediment. Another explanation could be that the worms had a lower tissue concentration because they ventilate their burrows with the overlaying water, which had a lower chemical activity than the sediments (the water was aerated and partially exchanged with fresh seawater every $3 \mathrm{~d}$ ). Although polychaetes accumulate contaminants mostly through ingestion of sediment, as opposed to dermal transfer, ${ }^{52-54}$ it is possible that the measured body burden is not the concentration at equilibrium with the sediment, but rather a steady state concentration resulting from uptake (ingestion, dermal transfer) and elimination processes (egestion and respiration). 

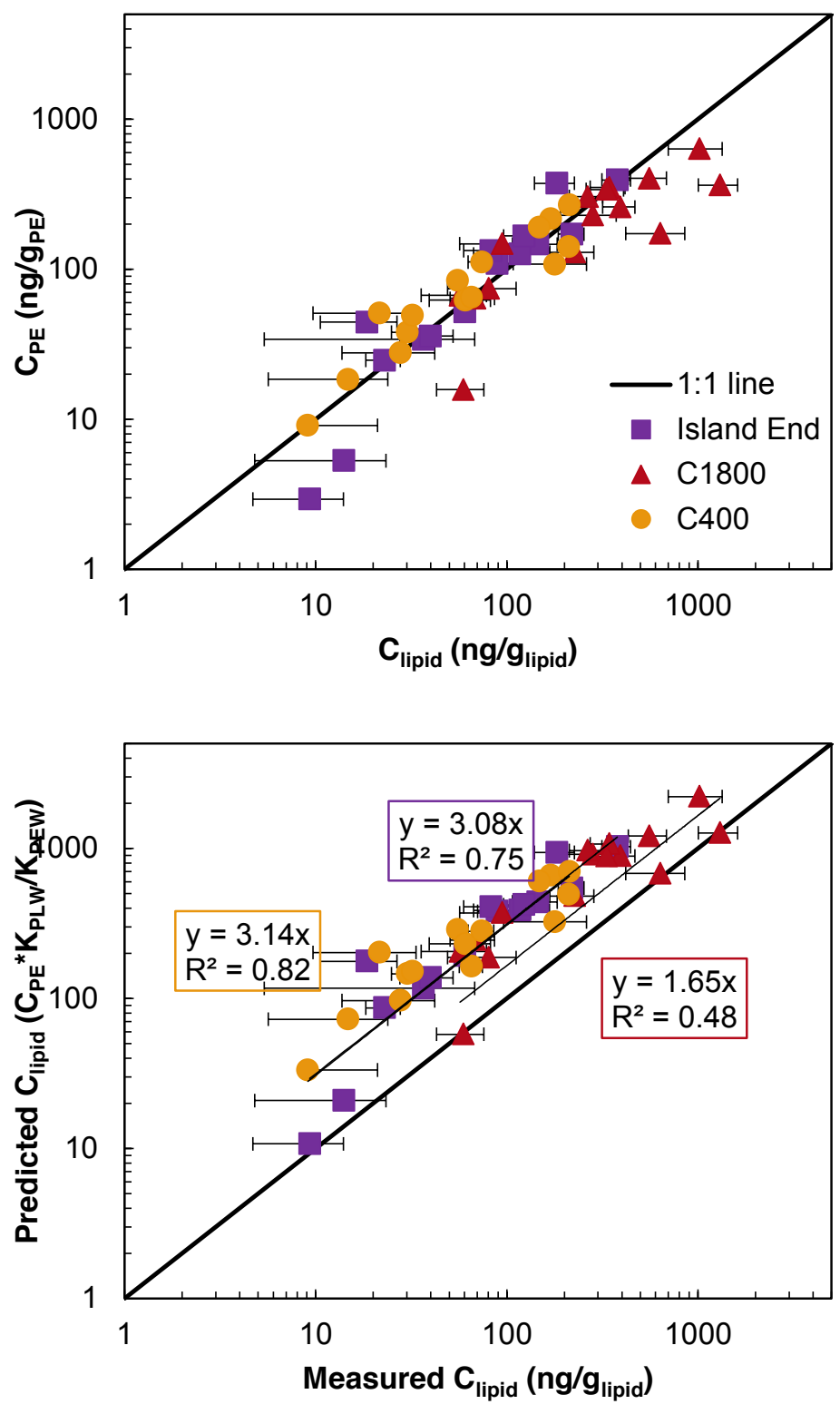

Figure 3.8 (Top) Concentration of PCBs in PE and in the corresponding worm tissue for Island End, C1800 and C400 test sediments. (Bottom) Lipid normalized tissue concentration of PCBs calculated from measured concentrations in tumbled PE and $\mathrm{GC} \times \mathrm{GC}$ derived partition $K_{P L-P E}$, versus measured concentrations in worms incubated with same sediments. Also shown are the linear fits for each sediment and the 1:1 line. 
Even though the PE-based estimations and tissue concentrations were not in perfect agreement in our study, the agreement within a factor of 2-3 is comparable to previous studies. In a similar study by Friedman et al. ${ }^{55}$ the authors observed concentrations of PCBs and in PE that were close to 1:1 line, and similarly found that PE-based calculations of bioaccumulation overestimated the tissue concentration by 3.6 times, on average. In contrast, Gschwend et al. ${ }^{48}$ used a different polychaete, Neanthes arenaceodentata, and found concentrations in organisms which were $5.3 \pm 0.9$ times larger than in the PE. Results of other studies employing different benthic invertebrates and PDMS passive samplers found PCB concentrations that were 5 to 10 fold higher in the lipids. ${ }^{56-58}$ The referenced studies used either equilibrium passive sampling or adjusted for disequilibrium in kinetic sampling, so it is unlikely that the observed factors reflect under-equilibrated sampler concentration. Thus, the concentration in the biota may not always reflect thermodynamic equilibrium, and depending on species and the testing conditions (impacts of the sediment quality and contamination on the physiology of the test organisms), the samplerbased predictions may only be at best within a factor of 3-5 of the measured bioaccumulation. For example, the accumulation of contaminants in the tissue of Nereis virens could affect the feeding rate of the organism or their rate of respiration, which could overall lead to body burdens that are different than equilibrium-based predictions.

PAHs. Concentrations of 16 PAHs were measured in worm tissue, PE and sediments (Tables S7-S10) and the concentrations of 4 ring and larger PAHs were all several orders of magnitude higher in the exposed worms compared to the blank ones, confirming that the PAH loads in the tissues were due to exposure to sediment as opposed to preexisting contamination in the worms. Naphthalene and phenanthrene were present at detectable levels in the procedure blanks (50-100 ng/g lipid or PE, Table S6), and small PAHs with 2, 3 and 4 rings were present in the blank worms at 20-170 $\mathrm{ng} / \mathrm{g}_{\text {lipid }}$ (phenanthrene at $1400 \mathrm{ng} / \mathrm{g}_{\text {lipid, }}$ Table S6). Accumulation of naphthalene in worms was not significant because it was not above the background level measured in the procedure blanks, and thus naphthalene was excluded from the following discussion. Phenanthrene and fluorene were the only PAH whose concentrations were higher in the blank worms $\left(1400\right.$ and $\left.130 \mathrm{ng} / \mathrm{g}_{\text {lipid }}\right)$ than in the test worms $\left(160-400 \mathrm{ng} / \mathrm{g}_{\text {lipid }}\right.$ and 40-100 $\left.\mathrm{ng} / \mathrm{g}_{\text {lipid }}\right)$. 
The concentrations of PAHs in PE were well above detection limits, and reflected the contamination in sediment but also the importance of organic and black carbon phases in controlling bioavailability. Although Island End sediment had a total PAH concentration (sum of 16 PAHs measured and listed in Tables S7-S10, with the exception of naphthalene) almost an order of magnitude higher than C400 (300 versus $50 \mu \mathrm{g} / \mathrm{g}_{\mathrm{dw}}$ ), the PAHs accumulated to similar extents in the PE from both sediments ( 970 and $1200 \mu \mathrm{g} / \mathrm{g}_{\mathrm{PE}}$, respectively). This is likely because of the lower $f_{O C}$ and $f_{B C}$ of the $\mathrm{C} 400$ sediment (Table 3.2). PE and worms incubated in Island End and C400 accumulated almost an order of magnitude more PAHs than those incubated in 303.1 and C1800 (Table 3.2), which is again consistent with the organic and black carbon contents of these sediments. C1800 sediment had similar PAH concentrations but a higher $f_{B C}$ compared to C400 (30 and $52 \mu \mathrm{g} / \mathrm{g}_{\text {sed }}$ and 1.9 and $0.32 \%$, respectively, Table 3.2). For 303.1, the PAH load was low in the sediment $\left(5 \mu \mathrm{g} / \mathrm{g}_{\text {sed }}\right)$ but the contaminants were likely more bioavailable due to the low $f_{O C}$ and $f_{B C}(1.28$ and $0.19 \%$, Table 3.2). For both the worms and the PE, pyrene and fluoranthene had the highest concentrations of the 16 PAHs measured, accounting for $50-60 \%$ of the total PAH load.

The lipid normalized PAH concentration measured in the worms was on average 0.07 times lower than the measured accumulation in PE (range 0.01 to 0.5, Figure 3.9). Employing the GC $\times$ GC-calculated $K_{P L-P E}$ values (Figure 3.6) and evaluating the expected concentration of PAHs in lipids based on concentration in PE samplers, resulted in over-prediction of PAH concentrations in worms by factors of 10 to 300 (median 170). It is worth noting that, similarly to the PCB results, the comparison between $\mathrm{PE}$ and worm concentrations was most favorable for $\mathrm{C} 1800$ (predicted/measured median ratio of 90), implying that perhaps, the worms incubated with $\mathrm{C} 1800$ had higher ingestion rates, higher assimilation efficiencies or lower elimination rates. As was the case for PCBs, the exposure time of $28 \mathrm{~d}$ was previously found to be sufficient for PAHs to reach steady state concentrations in the tissue of Nereis virens, ${ }^{11}$ so we do not believe that longer incubations of the worms in the sediment would have led to higher accumulation of PAHs in the worms. 
Expt $\mathrm{K} \diamond \mathrm{GC} \times \mathrm{GCK} \Delta 303.1 \times \mathrm{C} 400 \times \mathrm{C} 1800$ elsland End

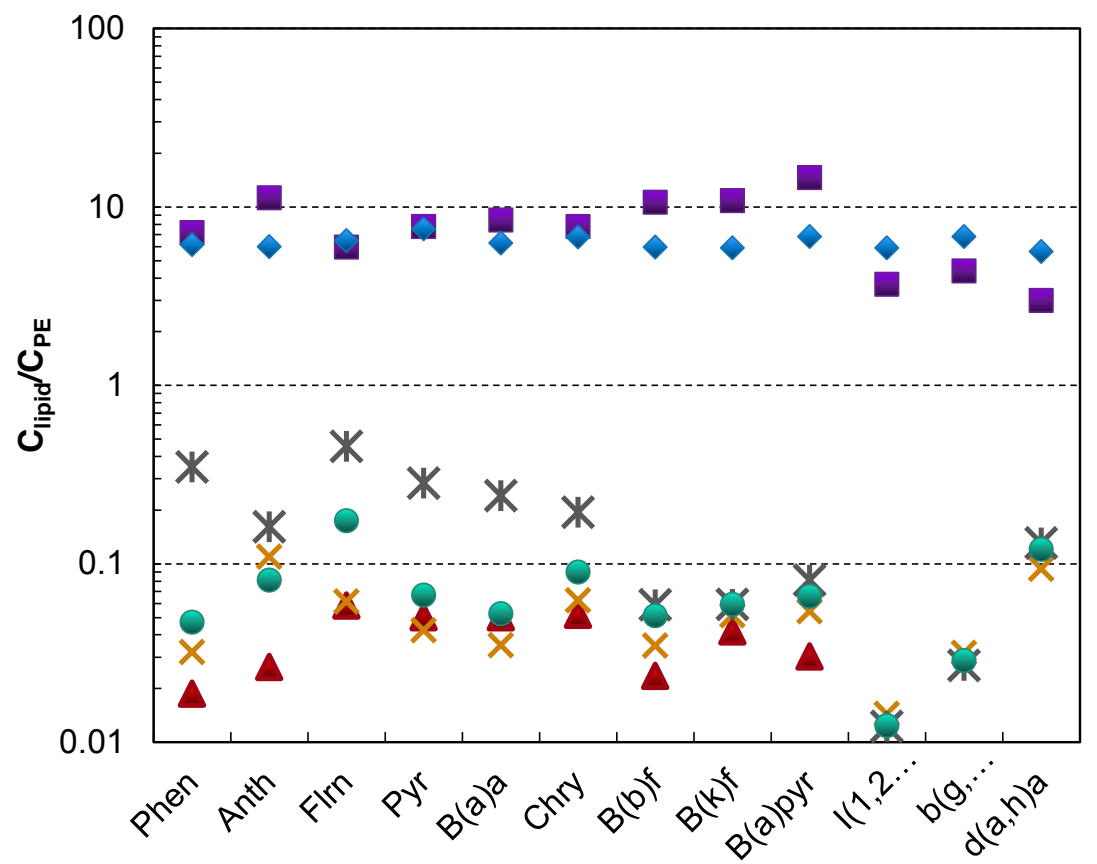

Figure 3.9 Ratio of concentration measured in lipid over concentration measured in PE for various PAHs $($ Phen $=$ phenanthrene, Anth $=$ anthracene, Flrn $=$ fluoranthene, Pyr $=$ pyrene, $\mathrm{B}(\mathrm{a}) \mathrm{a}=\operatorname{benz}[a]$ anthracene, Chry $=$ chrysene, $\mathrm{B}(\mathrm{b}) \mathrm{f}=$ benzo[b]fluoranthene, $\mathrm{B}(\mathrm{k}) \mathrm{f}=$ benzo $[k]$ fluoranthene, $\mathrm{B}(\mathrm{a}) \mathrm{pyr}=$ benzo $[a]$ pyrene, $\mathrm{I}(1,2,3) \mathrm{p}=$ indeno[1,2,3-cd]pyrene, $\mathrm{b}(\mathrm{g}, \mathrm{h}, \mathrm{i},) \mathrm{p}=$ benzo[ghi]perylene, $\mathrm{d}(\mathrm{a}, \mathrm{h}) \mathrm{a}=\operatorname{dibenz}[a, h]$ anthracene $)$. Also displayed is the expected ratio at equilibrium evaluated as $\mathrm{K}_{\mathrm{PL}-\mathrm{PE}}=\mathrm{K}_{\mathrm{PLW}} / \mathrm{K}_{\mathrm{PEW}}$ calculated either based on $\mathrm{GC} \times \mathrm{GC}$ (blue diamonds, $\mathrm{GC} \times \mathrm{GC} \mathrm{K}$ ), or based on experimental values in the literature (purple squares, Expt $\mathrm{K})$

The larger disagreement between PE-based predictions and measured accumulation in the worms for PAHs $(10-130 \times)$ versus PCBs $(2-3 \times)$ suggests that degradation processes prevented complete equilibration between the worm lipids and the sediment. Unlike PCBs, PAHs are more susceptible to degradation in the gut of Nereis virens, but also with light in the overlaying water and by bacteria in the sediments. In the case of photodegradation, the ratio of pyrene/fluoranthrene should be higher in the worm tissue versus measured in the sediment or PE, as fluoranthene has higher photolysis rates. ${ }^{60}$ Since the ratio of pyrene/fluoranthene remained relatively constant in the three different media ( 2-4 for C1800 and $\sim 0.7$ for the other three sediments), direct photolysis most likely did not cause the observed lower concentrations in the worms compared to PE-based predictions. In addition, as mentioned before, as a contaminant accumulation route, dermal uptake from overlaying water is small in comparison with sediment 
ingestion for Nereis virens. We also considered the effect of microbial degradation of the PAHs in the sediment during the $28 \mathrm{~d}$ test period, but we do not believe that it could have caused the disagreement between worm tissue concentrations and PE-based estimates. Since the worms accumulated 10 to 100 times less than predicted assuming chemical equilibrium of lipid tissue and the sediment, this would imply half lives of PAHs in the test sediments on the order of 1 to 10 days. While such fast degradation rates have been observed for small PAHs like naphthalene, benzothiophenes and derivatives, ${ }^{61}$ the rates for PAHs larger than phenanthrene are typically much smaller (half-lives of 30-300 days). ${ }^{62}$

Thus, PAH metabolism by Nereis virens or its associated gut microflora is the most likely explanation for the observed disequilibrium between the concentration measured in the worm tissue and the PE. Nereis virens is known to be efficient at metabolizing PAHs (e.g., after a 5 day exposure, only 17 and $24 \%$ of total accumulated pyrene ${ }^{63}$ and benz $[a]$ anthracene, ${ }^{64}$ respectively, were still found as parent $\mathrm{PAH}$, as opposed to polar or conjugated metabolites, in the Nereis virens tissue). After 28 days, the fraction of benz[a]anthracene further decreases to $5 \%,{ }^{64}$ although other studies measured a rather slow decline in parent PAH concentration (at 28 days concentrations in tissue only decreased by $10-30 \%$ compared to 5 days). ${ }^{11}$ If the total PAH (including polar and conjugate forms, 4-20× the measured parent $\mathrm{PAH}$ ) is representative of the concentration of PAH that would have accumulated in the tissue in the absence of metabolism, this implies that PE-based predictions agree to within a factor of 8 with theoretical nometabolism $\mathrm{PAH}$ concentrations in the worms. It is also generally thought that $\mathrm{PAH}$ transformation rates are inversely proportional to $\mathrm{PAH}$ size, implying greater discrepancy between measured tissue concentrations and PE-based predictions for smaller PAHs. This trend was apparent for the $\mathrm{C} 1800$ sediment, where the ratio of $\mathrm{C}_{\text {lipid }} / \mathrm{C}_{\mathrm{PE}}$ was positively correlated with $K_{O W}$ (correlation coefficient -0.68), but not for the other sediments (correlation coefficients close to 0). The ability of Nereis virens to degrade PAHs was found to be independent of the concentrations of contaminants in the sediment. ${ }^{65}$ This is consistent with our observations $\Sigma \mathrm{PAH}$ concentrations in test sediments varied from 5 to $300 \mathrm{ug} / \mathrm{g}_{\mathrm{dw}}$. (Table 3.2), but PAH concentration in the worms incubated in the least contaminated sediment (303.1) were not in significantly better agreement with equilibrium-based prediction than the more contaminated sediments (Figure 3.9). Altogether, PAH metabolism in Nereis virens appears to have impacted 
the measured PAH load in the exposed worms and accounted for, at least in part, the disagreement with PE-based estimates.

Thus, employing passive sampling for evaluating bioaccumulation of PAHs in benthic polychaetes will likely give upper estimates of the concentrations expected in the tissue of invertebrates. Many studies 9, 11,66-68 have found that body burdens of PAHs in Nereis virens and other invertebrates (Hinia reticulata, Limbriculus variegatus) are usually overestimated based on passive sampler accumulation by factors of 2 to 10 or even higher. This is not surprising given that many invertebrates, or their associated microbes, metabolize PAHs, although it should be noted that the transformation rates are strongly dependent on the species. ${ }^{63,65,69}$ Although Nereis virens was chosen for this experiment for its relatively large size, which would provide low detection limits for a variety of HOCs, other polychaetes which do not metabolize PAHs would be more appropriate to use in the future, such as Leitoscolopus fragilis. ${ }^{65}$ Rust et al. ${ }^{69}$ showed that amphipods like Leptocheirus plumulosus or Ampelisca abdita have a reduced ability to metabolize PAHs compared to numerous other polychaetes, but detection limits will be higher because of their smaller size.

$D D X s$. DDT and its degradation products DDE and DDD were measurable only in worms and PE exposed to the sediments from San Francisco Bay (Table 3.2). The 4,4' isomers comprised the bulk of total DDX concentrations, with 2,4'-DDT and 2,4'-DDD accounting for less than $20 \%$ of $\Sigma$ DDX, and 2,4'-DDE measurable only in PE but accounting for less than 1\% of $\Sigma$ DDX. Concentrations of DDX were close to detection limits for the reference site (303.1) in all three matrices (PE, worm tissue and sediment) and thus, only results from C400 and C1800 tests will be discussed. Total DDX concentration in C1800 sediment was 8-fold higher than in the C400 sediment (Table 3.2). However, the $\mathrm{DDDX}$ in the PE equilibrated with $\mathrm{C} 1800$ sediment was only 2 times more than in the PE equilibrated with C400 sediment, illustrating again the effect of organic carbon on availability on the contaminants' freely dissolved concentrations (C1800 sediment had 3 times more OC and 6 times more BC than C400, Table 3.2). A similar trend is observed when looking at individual contaminants such as 2,4'-DDD, which was 10 times more abundant in the C1800 than in the C400 sediment, but only 3 times more abundant in the respective PE strips incubated with the same sediments (Tables S8 and S9). Interestingly, the 
worms incubated in the two sediments did not show the same trend (factor of 6 difference between $\Sigma$ DDX and factor of 7 for 2,4'-DDD in C1800 versus C400 worm tissue, Table 3.2).

Relative to the sediment, both the worm tissue and the PE were depleted in the parent compound (DDT) but enriched in DDD in the case of the worm tissue, and in DDE in the case of PE. This was the case for both C400 and C1800 sediments (Figure 3.10). Based on the parent DDT concentration in each sediment, we expected concentrations of DDT in the tumbled PE between 100-1000 ng/ge. But interestingly, for both sediments, the tumbled PE did not accumulate any measurable amount of DDT $\left(<5 \mathrm{ng} / \mathrm{g}_{\mathrm{PE}}\right)$. Instability of DDT in sediment slurries has been observed previously (Chapter 6 , other studies ${ }^{70-72}$ ). While these other studies observed mainly transformation in sediment slurries of DDT to DDD, the proportional increase in DDE relative to DDD in PE (which is also apparent for the 2,4' isomers in C1800), suggests that DDT may have been degraded during the incubation with PE, and produced DDE, in addition to DDD. Assuming that all DDT degraded to DDE and DDD, and that partitioning from sediment into PE is twice as large for DDE as it is for DDD (based on measured ratios in PE and in sediment for the lake sediment in Chapter 6), we estimate that during the PE-sediment incubation about $\sim 15$ $\%$ of DDT transformed to DDE and $\sim 85 \%$ transformed to DDD.
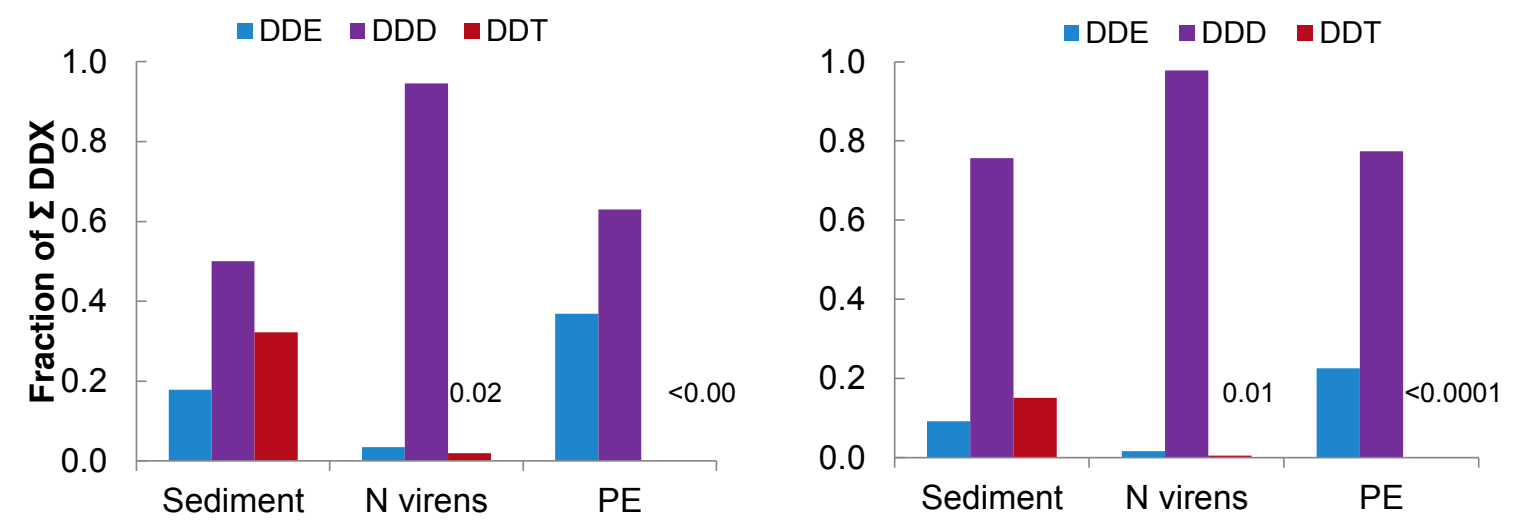

Figure 3.10 Fraction of 4,4'-DDE, 4,4'-DDD and 4,4'-DDT of total 4,4'-DDX in the sediment, $N$. virens tissue and $\mathrm{PE}$ for $\mathrm{C} 400$ (left) and $\mathrm{C} 1800$ (right). Text refers to the fraction of parent compound DDT in the $\mathrm{C} 400$ and $\mathrm{C} 1800$ worm tissue: 0.02 and 0.01 , respectively, and the maximum fractions in PE: $<0.002$ and $<0.0001$, respectively. DDT was below detection in both the $\mathrm{C} 400$ and $\mathrm{C} 1800 \mathrm{PE}$.

In contrast, in the bioaccumulation experiments, DDT concentrations were well above detection limits in the worm tissue (200-500 ng/g lipid), but DDT accounted for less than $2 \%$ of 
the $\Sigma \mathrm{DDX}$ in the worm tissues. If the reactions degrading DDT would be taking place only in the sediment, as was the case for PE incubations, then we would have similarly expected to see DDT concentrations below detection limits. However, the presence of the parent compound in the worm tissue indicates that the worm is actively ingesting sediment that still contains DDT, and the increased proportion of DDD and decreased proportion of DDE suggest that a transformation of DDT to DDD is taking place in the gut of Nereis virens. Although we did not measure DDX concentrations in sediments at the end of the bioaccumulation or PE tumbling experiments, other studies found that the degradation of DDT is faster when the sediment is mixed, than during static incubations like the bioaccumulation experiments, ${ }^{71}$ as would be the case if degradation was limited by desorption rates. ${ }^{73}$ Although to our knowledge, no studies looked specifically at transformation of DDT by Nereis virens, transformations in other biota have been documented. The degradation product(s) were however, species specific: DDD in Nereis succinea ${ }^{71}$ and other invertebrates, ${ }^{74}$ DDE in the freshwater amphipod Hyalalla azteca, ${ }^{75}$ and both DDE and DDD in Neanthes arenaceodentata. ${ }^{76}$

Despite the DDT reactivity in both PE and worm incubations, the data from the C1800 test sediment were still suitable for comparing $\mathrm{GC} \times \mathrm{GC}$ predictions against empirical observations of bioaccumulation. In C1800 sediment, the DDD concentration is 5 times that of DDT, meaning that even if reactions were transforming all DDT to DDD, the DDD concentration would only be affected by $20 \%$. Comparing the ratios of measured concentration in the lipids of $N$. virens and in the tumbled PE, against the predictions based on $\mathrm{GC} \times \mathrm{GC}$ retention times, we find generally good agreement for both 2,4'- and 4,4'-DDD (within a factor of 1.4). Even for C400, the agreement is within a factor of 2, possibly because similar amounts of DDD (limited by DDT concentration in sediment) were produced in both the worm tissue and the PE-sediment incubation. In contrast, the measured PE-lipid ratio for DDE is smaller than the $\mathrm{GC} \times \mathrm{GC}$ or literature based estimations (see caption of Figure 3.11), most likely due to the production of DDE during PE-sediment incubations which overestimated DDE availability. Accumulation of DDE in PE was likely higher than it should have been at equilibrium with the sediment, due to the additional DDE produced from DDT in the PE-sediment incubations.

Lastly, the presence of parent DDT in the sediment (10-20\%), is indicative of a more recent source of DDT to the Lauritzen Channel sediments, as was found also by other investigations at 
the site. ${ }^{33}$ We note though that a few years have passed since the collection of this sediment (2012) and its use in the bioaccumulation study (2014) described herein, and given DDT's reactivity, the pattern of contamination observed in this study may not be reflective of the original conditions in the freshly collected sediment. The percentage of parent DDT in the sediments used in this study was $10-20 \%$, which is much lower than the abundance of DDT in technical grade DDT (65-80\%). At the same time, sampling ${ }^{33}$ of the surface sediments of Lauritzen Channel in 2013 found abundances of DDT ranging from 30 to $65 \%$, closer to the value in technical grade DDT.

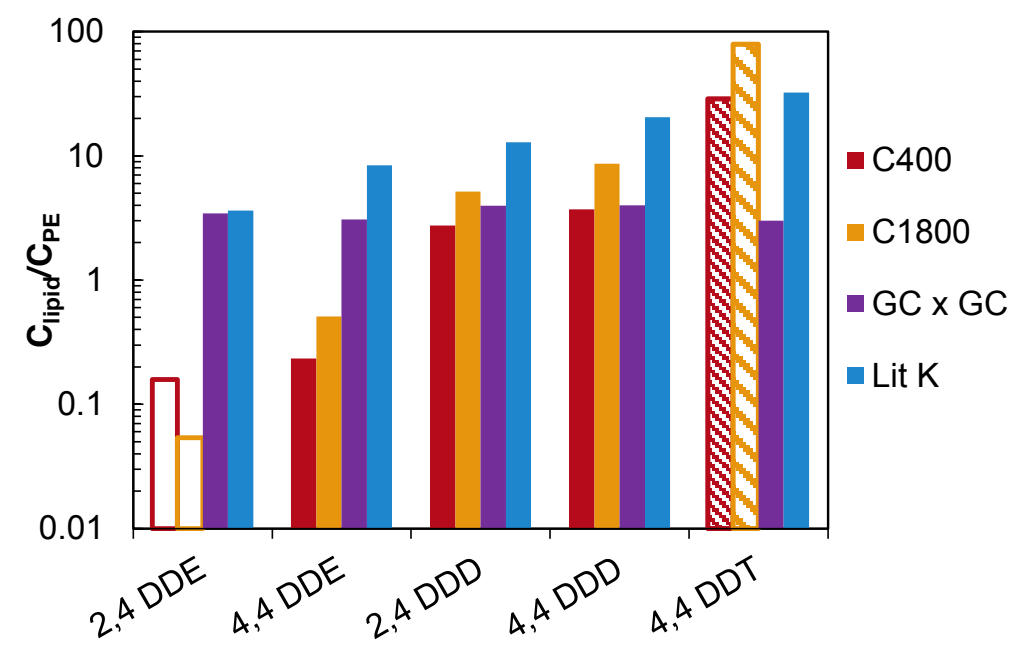

Figure 3.11 Comparison of measured ratios of concentration in worms divided by concentration in PE for various DDXs for $\mathrm{C} 400$ and $\mathrm{C} 1800$ experiments, against $\mathrm{GC} \times \mathrm{GC}$ predictions and literature based (Lit K, with $K_{P E W}$ from Hale et al. ${ }^{77}$ and $K_{P L W}$ based on LFER of Endo et al. ${ }^{42}$ and $K_{O W}$ values ${ }^{47}$ ) estimates of $K_{P L-P E}$ for the same compounds. Empty bars refer to lower estimates as DDE concentration in the worm tissue was below detection. Oblique pattern fill represents upper estimates because DDT concentration in PE was below detection.

$U C M$ and $G C \times G C-F I D$ analysis. Unresolved complex mixture traces were present in both Island End and C1800 PE samples. Most of the UCM in Island End PE eluted between alkanes with 12 to 37 carbon atoms $(n \mathrm{C} 12-n \mathrm{C} 37, \sim 40-80 \mathrm{~min}$, Figure 3.12$)$ while in $\mathrm{C} 1800 \mathrm{PE}$, the later eluting UCM $(n \mathrm{C} 25-n \mathrm{C} 35)$ dominated over the early portion $(n \mathrm{C} 15-n \mathrm{C} 22)$. The integrated load of HOCs in PE, calculated from a blank-subtracted chromatogram and with an average response factor based on alkane, PAH and alkyl benzene standards, was a factor of 10 higher in Island End compared to C1800 PE (56 and $5 \mathrm{mg} / \mathrm{g}_{\mathrm{PE}}$, respectively). 

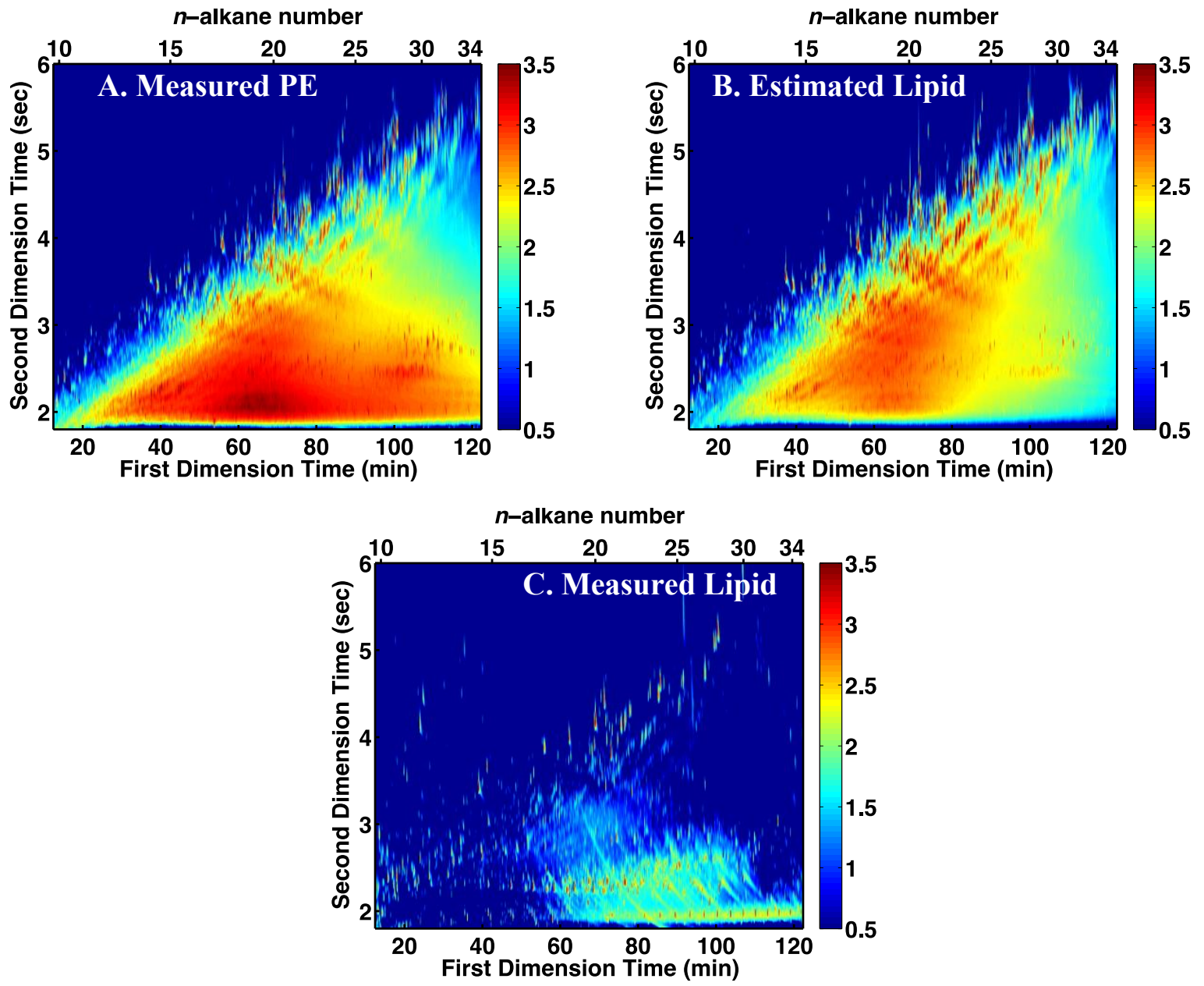

Figure 3.12 GC $\times$ GC - FID chromatograms of PE extract (A) and the worm tissue (C) incubated in Island End sediment. Panel B shows the estimated concentrations in the lipids, assuming chemical equilibrium between PE-sediment-lipids. The color represents the concentration $(\mathrm{ng} / \mathrm{g})$ at each point in the chromatogram.

Combining the PE chromatograms with equations for evaluating $K_{P E W}$ and $K_{P L W}$ from $\mathrm{GC} \times$ GC retention times (Table 3.1, Equations 3.10-3.11), we calculated the distribution and concentration of the UCM trace in the lipids of an organism at equilibrium with the sediment. The integrated lipid loads were $\sim 20 \%$ higher than the measured PE loads (69 and $7 \mathrm{mg} / \mathrm{g}$ lipid for Island End and C1800 PE). Consistent with the $K_{P L-P E}$ contours in Figure 3.6, the estimated lipid concentrations of PAHs and the moderately polar components of the UCM $\left(R T_{2}>3 \mathrm{~s}\right)$ were larger than measured in the PE. In contrast, the concentration of the saturated portion of the UCM (closer to $n$-alkane line, $R T_{2} 2-3 \mathrm{~s}$ ) was attenuated in lipids compared to PE by $\sim 10$ fold (Figure 3.12). 
However, despite the expectation that UCM hydrocarbons from Island End should be present at $\sim 10-100 \mathrm{ng} / \mathrm{g}_{\text {lipid }}$ (Figure 3.12) which is well above the detection limit of the GC $\times$ GC FID $(\sim$ $0.5 \mathrm{ng} / \mathrm{g}_{\text {lipid }}$ ), only a moderately polar portion of the UCM was measurable in the lipids of $N$. virens. Furthermore, this portion of the $\mathrm{UCM}\left(n \mathrm{C} 18-n \mathrm{C} 23, T_{2} 2.5-3.5 \mathrm{~s}\right.$, Figure 3.12) was only present at levels that were 10-100 times lower than PE-based predictions. Since the FID is a nonspecific detector, GC $\times$ GC-TOFMS analysis was used to confirm that similar compounds were present in the UCM elution window in both the PE and lipids. Based on library searches with match factors greater than 700 (i.e., peaks that could be assigned a structure based on the library search, not necessarily the quantitatively dominant peaks), the elution window in question $\left(n \mathrm{C} 18-n \mathrm{C} 23, T_{2} 2.5-3.5 \mathrm{~s}\right)$ included, in the PE, compounds containing both aromatic and aliphatic cycles, like derivatives of dibenzothiophene (Figure S7). However, the presence of dibenzothiophene derivatives could not be confirmed in the worm extract with GC $\times$ GCTOFMS analysis (even though the corresponding area of the chromatogram with GC $\times$ GC FID analysis showed a measurable signal, Figure 3.12) mostly likely because the signal was below detection limit of the TOFMS detector. For C1800, the concentrations of UCM compounds were below detection in the worm tissues when analyzed with either the FID or the TOFMS detectors, suggesting that the behavior of the UCM compounds was similar between the two sites (a 10 fold lower concentration in $\mathrm{C} 1800$ incubated worms compared to Island End worms, as expected based on the 10 fold lower concentration in C1800 PE compared to Island End PE, would render HOC concentrations below detection in the $\mathrm{C} 1800$ incubated worms).

Lastly, for both Island End and C1800, the portion of low polarity UCM $\left(T_{2} 2-2.5 \mathrm{~s}, n \mathrm{C} 23-\right.$ $n \mathrm{C} 34$ ) was confounded by the presence in the same retention window of biological material in the worm tissue extract (consistent with fatty acid methyl esters, Figure S8 and suggesting that breakthrough occurred during the silica column separation in section 3.2.5). GC $\times$ GC-TOFMS analyses confirmed though, that the characteristic ions present in this region in the PE (e.g., for $\mathrm{C} 1800$ a hydrocarbon signature with maximum $\mathrm{m} / \mathrm{z}$ of $69,83,94$ extending over $n \mathrm{C} 25-n \mathrm{C} 35$ and producing a broad peak in the second dimension of $\sim 1 \mathrm{~s}$ wide) were below the noise level in the corresponding worm tissue. Thus, this portion of the UCM $(n \mathrm{C} 25-n \mathrm{C} 35)$ behaved similarly to the earlier portion $\left(n \mathrm{C} 18-n \mathrm{C} 23, T_{2} 2.5-3.5 \mathrm{~s}\right)$ in that the accumulation in the worm was at least 100 times lower than predicted from the measured accumulation in the PE and the GC $\times \mathrm{GC}$ based $K_{P L W}$ and $K_{P E W}$ partition coefficients. 
Compared to extensive literature available for accumulation of PCBs, PAHs and other priority pollutants, studies that measured accumulation of UCMs in benthic species are scarce, ${ }^{78}$, ${ }^{79}$ and none compared UCM accumulation against an independent measure of bioavailability (e.g., passive sampling). Therefore, it is difficult to pinpoint whether the lower-than-predicted accumulation of UCM in the Nereis virens tissue was due to metabolism, or insufficient exposure time, or a combination of both. The partially aromatic portion of the UCM could be susceptible to the same metabolic processes as the PAHs, while the accumulation of more aliphatic and larger molecules may be limited by desorption rates from the sediment. ${ }^{80}$ The theory that uptake into biological tissue can be limited past a certain molecular size, has been recently challenged, ${ }^{81}$ and accumulation of hydrocarbons as large as $n \mathrm{C} 34$ has been observed in Lumbriculus variegatus after 28 day in petroleum contaminated sediments. ${ }^{79}$

\subsection{Summary and conclusions}

In the first part of this work, we developed a $\mathrm{GC} \times \mathrm{GC}$ based method for evaluating the partitioning between PE passive samplers and water. This method was able to estimate $K_{P E W}$ for hydrophobic organic chemicals (PAHs, PCBs, hydrocarbons and OCPs) with an average error of $0.4 \log$ units (factor of 3). When combined with the complementary relationship for estimating $K_{P L W}$ from Chapter 2, we were able to estimate how various HOCs partition between polymeric passive samplers and lipids, with the ultimate goal of using this to evaluate partitioning of HOC mixtures into biota. Across the $\mathrm{GC} \times \mathrm{GC}$ space, $K_{P L-P E}$ varied by two orders of magnitude and was strongly dependent on polarity. As such, partitioning of PAHs and PCBs preferred the lipid phase (factor of 6 for PAHs and 2-3 for PCBs), whereas more aliphatic components of HOC mixtures preferred the PE phase (e.g., $n$-alkanes factor of 3-5). For several PCBs and PAHs, there was good agreement between the $\mathrm{GC} \times \mathrm{GC}$ predictions of $K_{P L-P E}$ and parallel estimates based on literature values of $K_{P L W}$ and $K_{P E W}$. The advantage of the $\mathrm{GC} \times \mathrm{GC}$-based method is that it could be extended to other chemicals and technical mixtures for which there are fewer available experimental data in the literature.

In the second part, bioaccumulation experiments were used to check the $\mathrm{GC} \times \mathrm{GC}$ predictions for accumulation of PAHs, PCBs, DDXs and UCM compounds in tissue of Nereis virens and passive samplers. We found good agreement between sampler-based estimates and accumulation in the tissue of Nereis virens for PCBs (factor of 2-3) and for 2,4' and 4,4'-DDD 
(factor of 1.4). For PAHs, the PE-based estimates were 10-300 higher than the concentrations in worm tissue, which was most probably due to metabolism by the worm or its associated microflora. The UCM portion of the contaminants was also found at lower levels in the lipids compared to the PE (10-300 times less than predicted) and although little is known about the bioaccumulation kinetics of UCMs, it is possible that it was affected by both metabolism and slow desorption kinetics.

Nonetheless, equilibrium based estimates of biota body burdens, such as the one developed in this paper (e.g., accumulation in passive samplers combined with $\mathrm{GC} \times \mathrm{GC}$ based estimations of $\left.K_{P L-P E}\right)$, are advantageous because they (1) provide upper estimates for the concentrations that are likely to be found in the biota and (2) could serve as a starting point for assessing whether contaminants are being transformed and in which species. In addition, differences between

equilibrium-based estimations and measured tissue concentrations could also be indicative of physiological burdens on exposed organisms. The first approach is useful from a risk assessment perspective, because it gives the worst-case scenario. However, from an ecosystem perspective, distribution of contaminants across different benthic species can have implications on how contaminants migrate up the food chain and whether or not they lead to problematic body burdens in organisms from higher trophic levels such as fish.

\section{Acknowledgments}

We would like to thank Robert Burgess for valuable input regarding the experimental conditions of the bioaccumulation tests, Guilherme Lotufo for providing the San Francisco Bay sediment used in the bioaccumulation experiments, John MacFarlane for assistance with Island End sediment collection and the MIT UROP office for financial support for JLB. 


\section{References}

1. US Environmental Protection Agency National Listing of Fish Advisories. http://fishadvisoryonline.epa.gov/Advisories.aspx

2. Wenning RJ, I. C., Summary of the SETAC Pellston Workshop on Use of Sediment Quality Guidelines and Related Tools for the Assessment of Contaminated Sediments. In Society of Environmental Toxicology and Chemistry (SETAC), Pensacola FL, USA. , 2002.

3. Kraaij, R.; Mayer, P.; Busser, F. J. M.; van het Bolscher, M.; Seinen, W.; Tolls, J.; Belfroid, A. C., Measured pore-water concentrations make equilibrium partitioning work a data analysis. Environ Sci Technol 2003, 37, (2), 268-274.

4. Cornelissen, G.; Breedveld, G. D.; Naes, K.; Oen, A. M. P.; Ruus, A., Bioaccumulation of native polycyclic aromatic hydrocarbons from sediment by a polychaete and a gastropod: Freely dissolved concentrations and activated carbon amendment. Environ Toxicol Chem 2006, $25,(9), 2349-2355$.

5. Morrison, H. A.; Gobas, F. A. P. C.; Lazar, R.; Haffner, G. D., Development and verification of a bioaccumulation model for organic contaminants in benthic invertebrates. Environ Sci Technol 1996, 30, (11), 3377-3384.

6. Mayer, P.; Parkerton, T. F.; Adams, R. G.; Cargill, J. G.; Gan, J.; Gouin, T.; Gschwend, P. M.; Hawthorne, S. B.; Helm, P.; Witt, G.; You, J.; Escher, B. I., Passive sampling methods for contaminated sediments: scientific rationale supporting use of freely dissolved concentrations. Integr Environ Assess Manag 2014, 10, (2), 197-209.

7. US Environmental Protection Agency Equilibrium partitioning sediment benchmarks (ESBs) for the protection of benthic organisms: Procedures for the determination of the freely dissolved interstitial water concentrations of nonionic organics.; EPA/600/R-02/012; Washington, DC 204602012.

8. Greenberg, M. S.; Chapman, P. M.; Allan, I. J.; Anderson, K. A.; Apitz, S. E.; Beegan, C.; Bridges, T. S.; Brown, S. S.; Cargill, J. G. t.; McCulloch, M. C.; Menzie, C. A.; Shine, J. P.; Parkerton, T. F., Passive sampling methods for contaminated sediments: risk assessment and management. Integr Environ Assess Manag 2014, 10, (2), 224-36.

9. Vinturella, A. E.; Burgess, R. M.; Coull, B. A.; Thompson, K. M.; Shine, J. P., Use of passive samplers to mimic uptake of polycyclic aromatic hydrocarbons by benthic polychaetes. Environ Sci Technol 2004, 38, (4), 1154-1160.

10. Lydy, M. J.; Landrum, P. F.; Oen, A. M.; Allinson, M.; Smedes, F.; Harwood, A. D.; Li, H.; Maruya, K. A.; Liu, J., Passive sampling methods for contaminated sediments: state of the science for organic contaminants. Integr Environ Assess Manag 2014, 10, (2), 167-78.

11. Yates, K.; Pollard, P.; Davies, I. M.; Webster, L.; Moffat, C. F., Application of silicone rubber passive samplers to investigate the bioaccumulation of PAHs by Nereis virens from marine sediments. Environ Pollut 2011, 159, (12), 3351-3356.

12. Lohmann, R., Critical review of low-density polyethylene's partitioning and diffusion coefficients for trace organic contaminants and implications for its use as a passive sampler. Environ Sci Technol 2012, 46, (2), 606-18.

13. Sandermann, H., Jr., Differential lipid affinity of xenobiotics and natural compounds. Febs Lett 2003, 554, (1-2), 165-8.

14. Smedes, F.; Geertsma, R. W.; van der Zande, T.; Booij, K., Polymer-water partition coefficients of hydrophobic compounds for passive sampling: application of cosolvent models for validation. Environ Sci Technol 2009, 43, (18), 7047-54. 
15. Rusina, T. P.; Smedes, F.; Klanova, J.; Booij, K.; Holoubek, I., Polymer selection for passive sampling: a comparison of critical properties. Chemosphere 2007, 68, (7), 1344-51.

16. Booth, A. M.; Sutton, P. A.; Lewis, C. A.; Lewis, A. C.; Scarlett, A.; Chau, W.; Widdows, J.; Rowland, S. J., Unresolved complex mixtures of aromatic hydrocarbons: Thousands of overlooked persistent, bioaccumulative, and toxic contaminants in mussels. Environ Sci Technol 2007, 41, (2), 457-464.

17. Arey, J. S.; Nelson, R. K.; Xu, L.; Reddy, C. M., Using comprehensive two-dimensional gas chromatography retention indices to estimate environmental partitioning properties for a complete set of diesel fuel hydrocarbons. Anal Chem 2005, 77, (22), 7172-82.

18. Tcaciuc, A. P.; Nelson, R. K.; Reddy, C. M.; Gschwend, P. M., Estimating phospholipid membrane-water partition coefficients using comprehensive two-dimensional gas chromatography. Environ Sci Technol 2012, 46, (6), 3449-56.

19. deBruyn, A. M.; Gobas, F. A., The sorptive capacity of animal protein. Environ Toxicol Chem 2007, 26, (9), 1803-8.

20. Olive, P. J. W.; Craig, S.; Rutherford, G., Enhanced aquaculture feeds. In Google Patents: 2006.

21. Pocock, D. M. E.; Marsden, J. R.; Hamilton, J. G., Lipids in an intertidal polychaete and their relation to maturation of worm. Comp Biochem Physiol 1971, 39, (4A), 683-\&.

22. Hentschel, B. T., Spectrofluorometric quantification of neutral and polar lipids suggests a food-related recruitment bottleneck for juveniles of a deposit-feeding polychaete population. Limnol Oceanogr 1998, 43, (3), 543-549.

23. Nabi, D.; Gros, J.; Dimitriou-Christidis, P.; Arey, J. S., Mapping environmental partitioning properties of nonpolar complex mixtures by use of GC x GC. Environ Sci Technol 2014, 48, (12), 6814-26.

24. US Department of Commerce, National Oceanic and Atmospheric Administration, Sampling and Analytical Methods of the National Status and Trends Program Mussel Watch Project: 1993-1996 Update; NOAA Technical Memorandum NOS ORCA 130; Silver Spring, MD, 1998.

25. Choi, Y.; Cho, Y. M.; Luthy, R. G., Polyethylene-water partitioning coefficients for parent- and alkylated-polycyclic aromatic hydrocarbons and polychlorinated biphenyls. Environ Sci Technol 2013, 47, (13), 6943-50.

26. Adams, R. G.; Lohmann, R.; Fernandez, L. A.; Macfarlane, J. K.; Gschwend, P. M., Polyethylene devices: Passive samplers for measuring dissolved hydrophobic organic compounds in aquatic environments. Environ Sci Technol 2007, 41, (4), 1317-1323.

27. Fernandez, L. A.; MacFarlane, J. K.; Tcaciuc, A. P.; Gschwend, P. M., Measurement of freely dissolved PAH concentrations in sediment beds using passive sampling with low-density polyethylene strips. Environ Sci Technol 2009, 43, (5), 1430-1436.

28. Hale, S. E.; Martin, T. J.; Goss, K.-U.; Arp, H. P. H.; Werner, D., Partitioning of organochlorine pesticides from water to polyethylene passive samplers. Environ Pollut 2010, 158, (7), 2511-2517.

29. Gustafsson, Ö.; Gschwend, P. M., Hydrophobic organic compound partitioning from bulk water to the water/air interface. Atmos Environ 1998, 33, (1), 163-167.

30. CSID:20521. http://www.chemspider.com/Chemical-Structure.20521.html (15:56, Apr 24, 2015)

31. Abraham, M. H.; Poole, C. F.; Poole, S. K., Classification of stationary phases and other materials by gas chromatography. J Chromatogr A 1999, 842, (1-2), 79-114. 
32. US Environmental Protection Agency Overview of United Heckathorn Co. Superfund Site. $\quad$ http://yosemite.epa.gov/r9/sfund/r9sfdocw.nsf/BySite/United Heckathorn Co.?OpenDocument (accessed April 2015),

33. SEA Engineering, Inc. prepared for CH2M Hill under contract to EPA Region 9, United Heckathorn Superfund Site, Richmond, California DDT Fate and Transport Study; 2014. http://yosemite.epa.gov/r9/sfund/r9sfdocw.nsf/3dc283e6c5d6056f88257426007417a2/d9263fb3f 9c7358e88257d18005d365d/\$FILE/Final_Heckathorn_DDT_FateAndTransport.pdf (accessed April 2015)

34. Lee, H. I. B., B.L.; Pelletier, J.; Windsor, M.; Specht, D.T.; Randall, R.C. Guidance Manual: Bedded Sediment Bioaccumulation Tests; EPA-600/R-93-183; Office of Research and Development, USEPA: Washington, DC, 1993.

35. Kester, D. R.; Duedall, I. W.; Connors, D. N.; Pytkowicz, R. M., Preparation of artificial seawater. Limnol Oceanogr 1967, 12, (1), 176-179.

36. Kelly, B. C.; Fernandez, M. P.; Ikonomou, M. G.; Knapp, W., Persistent organic pollutants in aquafeed and Pacific salmon smolts from fish hatcheries in British Columbia, Canada. Aquaculture 2008, 285, (1-4), 224-233.

37. Bligh, E. G.; Dyer, W. J., A rapid method of total lipid extraction and purification. Can J Biochem Physiol 1959, 37, (8), 911-7.

38. Ewald, G.; Bremle, G.; Karlsson, A., Differences between Bligh and Dyer and Soxhlet extractions of PCBs and lipids from fat and lean fish muscle: Implications for data evaluation. Mar Pollut Bull 1998, 36, (3), 222-230.

39. Randall, R. C.; Lee, H.; Ozretich, R. J.; Lake, J. L.; Pruell, R. J., Evaluation of selected lipid methods for normalizing pollutant bioaccumulation. Environ Toxicol Chem 1991, 10, (11), 1431-1436.

40. Vernberg, F. J., Biological Monitoring of Marine Pollutants. Academic Press, Inc: 2012; p 114.

41. Pace, C. N.; Treviño, S.; Prabhakaran, E.; Scholtz, J. M., Protein structure, stability and solubility in water and other solvents. Philos T Roy Soc B 2004, 359, (1448), 1225-1235.

42. Endo, S.; Escher, B. I.; Goss, K. U., Capacities of membrane lipids to accumulate neutral organic chemicals. Environ Sci Technol 2011, 45, (14), 5912-21.

43. Sprunger, L.; Proctor, A.; Acree, W. E., Jr.; Abraham, M. H., Characterization of the sorption of gaseous and organic solutes onto polydimethyl siloxane solid-phase microextraction surfaces using the Abraham model. J Chromatogr A 2007, 1175, (2), 162-73.

44. Poerschmann, J.; Kopinke, F. D., Sorption of very hydrophobic organic compounds (VHOCs) on dissolved humic organic matter (DOM). 2. Measurement of sorption and application of a Flory-Huggins concept to interpret the data. Environ Sci Technol 2001, 35, (6), 1142-1148.

45. Barton, A. F. M., Handbook of Poylmer-Liquid Interaction Parameters and Solubility Parameters. CRC Press: 1990; 161.

46. Hawker, D. W.; Connell, D. W., Octanol-water partition coefficients of polychlorinated biphenyl congeners. Environ Sci Technol 1988, 22, (4), 382-387.

47. ATSDR Toxicological Profile for DDT, $D D E$ and DDD; US Department of Health and Human Services: 2002; pp 218-219. http://www.atsdr.cdc.gov/ToxProfiles/tp35.pdf

48. Gschwend, P. M.; MacFarlane, J. K.; Reible, D. D.; Lu, X.; Hawthorne, S. B.; Nakles, D. V.; Thompson, T., Comparison of polymeric samplers for accurately assessing PCBs in pore waters. Environ Toxicol Chem 2011, 30, (6), 1288-96. 
49. Fikslin, T. J.; Santoro, E. D., PCB congener distribution in estuarine water, sediment and fish samples: implications for monitoring programs. Environ Monit Assess 2003, 87, (2), 197212.

50. Anderson, B. S.; Hunt, J. W.; Phillips, B. M.; Stoelting, M.; Becker, J.; Fairey, R.; Puckett, H. M.; Stephenson, M.; Tjeerdema, R. S.; Martin, M., Ecotoxicologic change at a remediated superfund site in San Francisco, California, USA. Environ Toxicol Chem 2000, 19, (4), 879-887.

51. Bennett, E. R.; Steevens, J. A.; Lotufo, G. R.; Paterson, G.; Drouillard, K. G., Novel control and steady-state correction method for standard 28-day bioaccumulation tests using Nereis virens. Environ Toxicol Chem 2011, 30, (6), 1366-75.

52. Voparil, I. M.; Mayer, L. M., Dissolution of sedimentary polycyclic aromatic hydrocarbons into the lugworm's (Arenicola marina) digestive fluids. Environ Sci Technol 2000, 34, (7), 1221-1228.

53. Janssen, E. M.; Oen, A. M.; Luoma, S. N.; Luthy, R. G., Assessment of field-related influences on polychlorinated biphenyl exposures and sorbent amendment using polychaete bioassays and passive sampler measurements. Environ Toxicol Chem 2011, 30, (1), 173-80.

54. Leppanen, M. T.; Kukkonen, J. V. K., Relative importance of ingested sediment and pore water as bioaccumulation routes for pyrene to oligochaete (Lumbriculus variegatus, Muller). Environ Sci Technol 1998, 32, (10), 1503-1508.

55. Friedman, C. L.; Burgess, R. M.; Perron, M. M.; Cantwell, M. G.; Ho, K. T.; Lohmann, R., Comparing polychaete and polyethylene uptake to assess sediment resuspension effects on PCB bioavailability. Environ Sci Technol 2009, 43, (8), 2865-70.

56. Maaenpaa, K.; Leppanen, M. T.; Reichenberg, F.; Figueiredo, K.; Mayer, P., Equilibrium sampling of persistent and bioaccumulative compounds in soil and sediment: comparison of two approaches to determine equilibrium partitioning concentrations in lipids. Environ Sci Technol 2011, 45, (3), 1041-7.

57. You, J.; Landrum, P. E.; Trimble, T. A.; Lydy, M. J., Availability of polychlorinated biphenyls in field-contaminated sediment. Environ Toxicol Chem 2007, 26, (9), 1940-1948.

58. Lu, X.; Skwarski, A.; Drake, B.; Reible, D. D., Predicting bioavailability of PAHs and PCBs with porewater concentrations measured by solid-phase microextraction fibers. Environ Toxicol Chem 2011, 30, (5), 1109-16.

59. Sun, X.; Ghosh, U., The effect of activated carbon on partitioning, desorption, and biouptake of native polychlorinated biphenyls in four freshwater sediments. Environ Toxicol Chem 2008, 27, (11), 2287-95.

60. El-Saeid, M.; Al-Turki, A.; Nadeem, M. A.; Hassanin, A.; Al-Wabel, M., Photolysis degradation of polyaromatic hydrocarbons (PAHs) on surface sandy soil. Environ Sci Pollut $R$ 2015, 1-14.

61. Wardlaw, G. D.; Nelson, R. K.; Reddy, C. M.; Valentine, D. L., Biodegradation preference for isomers of alkylated naphthalenes and benzothiophenes in marine sediment contaminated with crude oil. Organic Geochemistry 2011, 42, (6), 630-639.

62. Park, K. S.; Sims, R. C.; Dupont, R. R.; Doucette, W. J.; Matthews, J. E., Fate of PAH compounds in two soil types: Influence of volatilization, abiotic loss and biological activity. Environ Toxicol Chem 1990, 9, (2), 187-195.

63. Jørgensen, A.; Giessing, A. M. B.; Rasmussen, L. J.; Andersen, O., Biotransformation of polycyclic aromatic hydrocarbons in marine polychaetes. Mar Environ Res 2008, 65, (2), 171 186. 
64. Mcelroy, A. E., Polycyclic aromatic hydrocarbon metabolism in the polychaete Nereis virens. Aquatic Toxicol 1990, 18, (1), 35-50.

65. Driscoll, S. K.; McElroy, A. E., Bioaccumulation and metabolism of benzo[a]pyrene in three species of polychaete worms. Environ Toxicol Chem 1996, 15, (8), 1401-1410.

66. Burkhard, L. P.; Mount, D. R.; Highland, T. L.; Hockett, J. R.; Norberg-King, T.; Billa, N.; Hawthorne, S. B.; Miller, D. J.; Grabanski, C. B., Evaluation of PCB bioaccumulation by Lumbriculus variegatus in field-collected sediments. Environ Toxicol Chem 2013, 32, (7), 14951503.

67. Oen, A. M. P.; Schaanning, M.; Ruus, A.; Cornelissen, G.; Kallqvist, T.; Breedveld, G. D., Predicting low biota to sediment accumulation factors of PAHs by using infinite-sink and equilibrium extraction methods as well as BC-inclusive modeling. Chemosphere 2006, 64, (8), $1412-1420$.

68. Barthe, M.; Pelletier, E.; Breedveld, G. D.; Cornelissen, G., Passive samplers versus surfactant extraction for the evaluation of PAH availability in sediments with variable levels of contamination. Chemosphere 2008, 71, (8), 1486-1493.

69. Rust, A. J.; Burgess, R. M.; Brownawell, B. J.; McElroy, A. E., Relationship between metabolism and bioaccumulation of benzo[a]pyrene in benthic invertebrates. Environ Toxicol Chem 2004, 23, (11), 2587-2593.

70. Bao, L. J.; Jia, F.; Crago, J.; Zeng, E. Y.; Schlenk, D.; Gan, J., Assessing bioavailability of DDT and metabolites in marine sediments using solid-phase microextraction with performance reference compounds. Environ Toxicol Chem 2013, 32, (9), 1946-1953.

71. Wang, F.; Pei, Y. Y.; You, J., Biotransformation of dichlorodiphenyltrichloroethane in the benthic polychaete, Nereis succinea: quantitative estimation by analyzing the partitioning of chemicals between gut fluid and lipid. Environ Toxicol Chem 2015, 34, (2), 360-8.

72. Gambrell, R. P.; Reddy, C. N.; Collard, V.; Green, G.; Patrick, W. H., The recovery of DDT, kepone, and permethrin added to soil and sediment suspensions incubated under controlled redox potential and pH conditions. $J$ Water Pollut Con F 1984, 56, (2), 174-182.

73. Rijnaarts, H. H. M.; Bachmann, A.; Jumelet, J. C.; Zehnder, A. J. B., Effect of desorption and intraparticle mass-transfer on the aerobic biomineralization of alpha-hexachlorocyclohexane in a contaminated calcareous soil. Environ Sci Technol 1990, 24, (9), 1349-1354.

74. Kwong, R. W. M.; Yu, P. K. N.; Lam, P. K. S.; Wang, W.-X., Biokinetics and biotransformation of DDTs in the marine green mussels Perna viridis. Aquatic Toxicol 2009, 93, (4), 196-204.

75. Lotufo, G. R.; Landrum, P. F.; Gedeon, M. L.; Tigue, E. A.; Herche, L. R., Comparative toxicity and toxicokinetics of DDT and its major metabolites in freshwater amphipods. Environ Toxicol Chem 2000, 19, (2), 368-379.

76. Lotufo, G. R.; Farrar, J. D.; Bridges, T. S., Effects of exposure source, worm density, and sex on DDT bioaccumulation and toxicity in the marine polychaete Neanthes arenaceodentata. Environ Toxicol Chem 2000, 19, (2), 472-484.

77. Hale, S. E.; Tomaszewski, J. E.; Luthy, R. G.; Werner, D., Sorption of dichlorodiphenyltrichloroethane (DDT) and its metabolites by activated carbon in clean water and sediment slurries. Water Research 2009, 43, (17), 4336-4346.

78. Porte, C.; Biosca, X.; Pastor, D.; Solé, M.; Albaigés, J., The Aegean Sea oil spill. 2. Temporal study of the hydrocarbons accumulation in bivalves. Environ Sci Technol 2000, 34, (24), 5067-5075. 
79. Muijs, B.; Jonker, M. T., Assessing the bioavailability of complex petroleum hydrocarbon mixtures in sediments. Environ Sci Technol 2011, 45, (8), 3554-61.

80. Kuo, D. T.; Adams, R. G.; Rudnick, S. M.; Chen, R. F.; Gschwend, P. M., Investigating desorption of native pyrene from sediment on minute- to month-timescales by time-gated fluorescence spectroscopy. Environ Sci Technol 2007, 41, (22), 7752-8.

81. Jonker, M. T.; Van der Heijden, S. A., Bioconcentration factor hydrophobicity cutoff: an artificial phenomenon reconstructed. Environ Sci Technol 2007, 41, (21), 7363-9. 
Supporting Information for:

Chapter 3 Evaluating bioaccumulation potential of HOC mixtures using GC $\times$ GC and passive sampling 
先

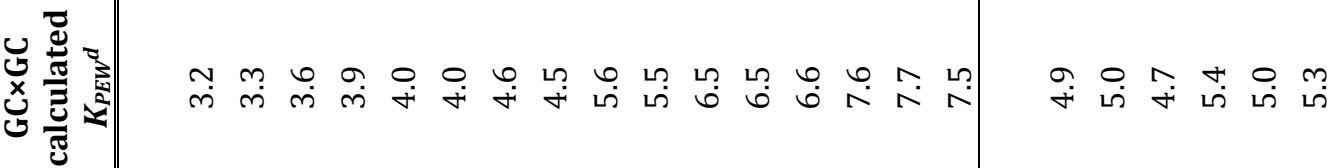

西

กั

$\hat{m}$

$\stackrel{0}{n+0}$

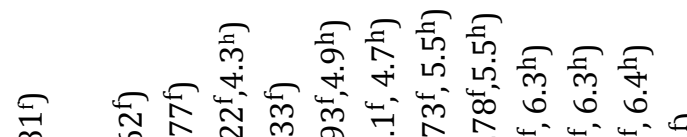

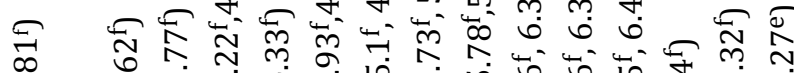

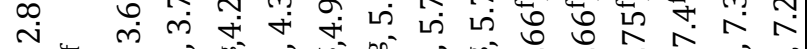

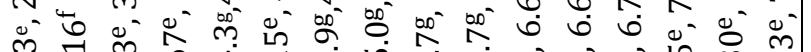

तु ने

ల్ర

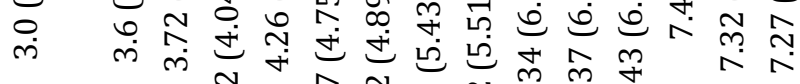

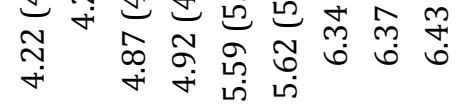

苛 考

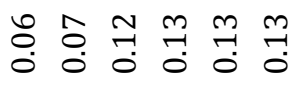

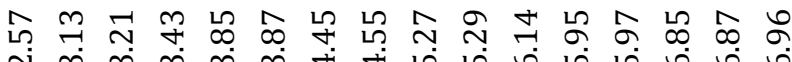

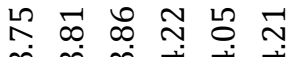

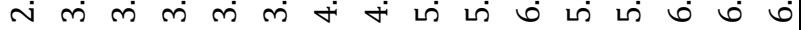

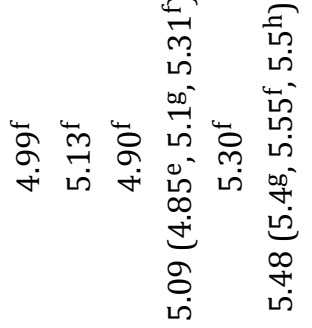

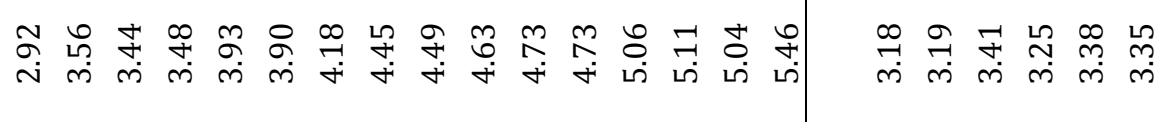

ह

$\frac{0}{\frac{0}{2}}$

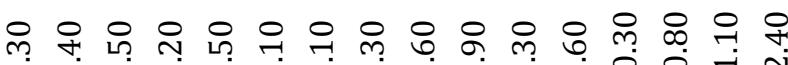

우 융유 우 तิ बे

苞

宅

छ

ํํㄹ

ํํㅇ

式

so $x$

$\Xi$ U

클

$\exists$

कs

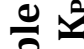

훙ㅇ

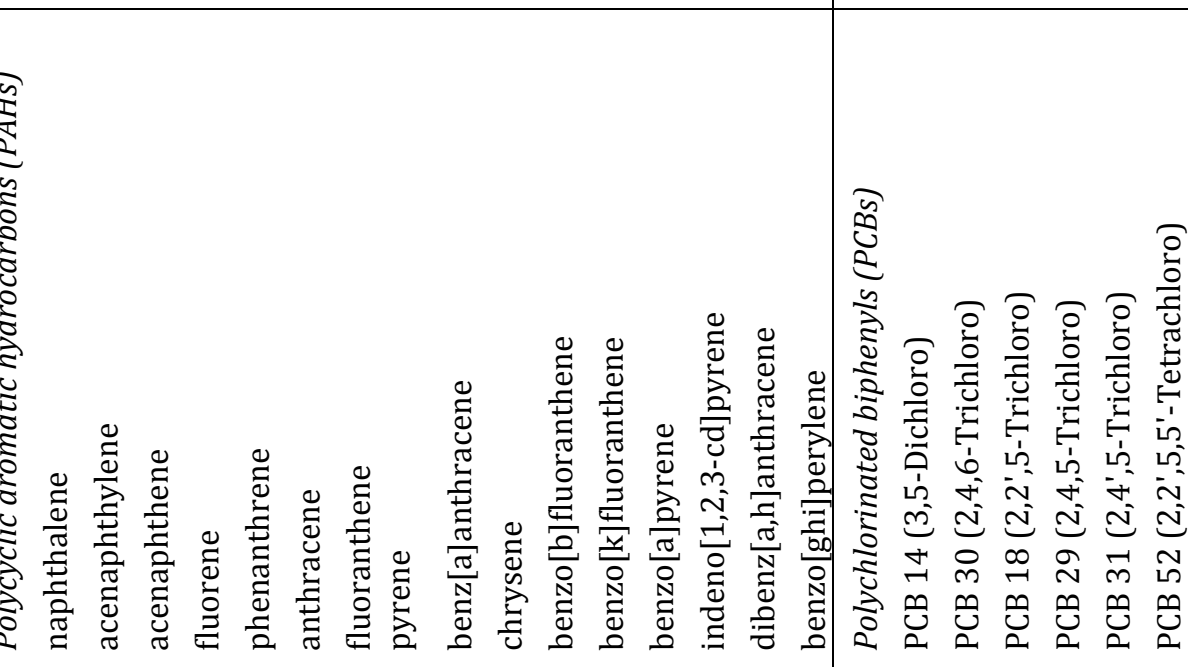




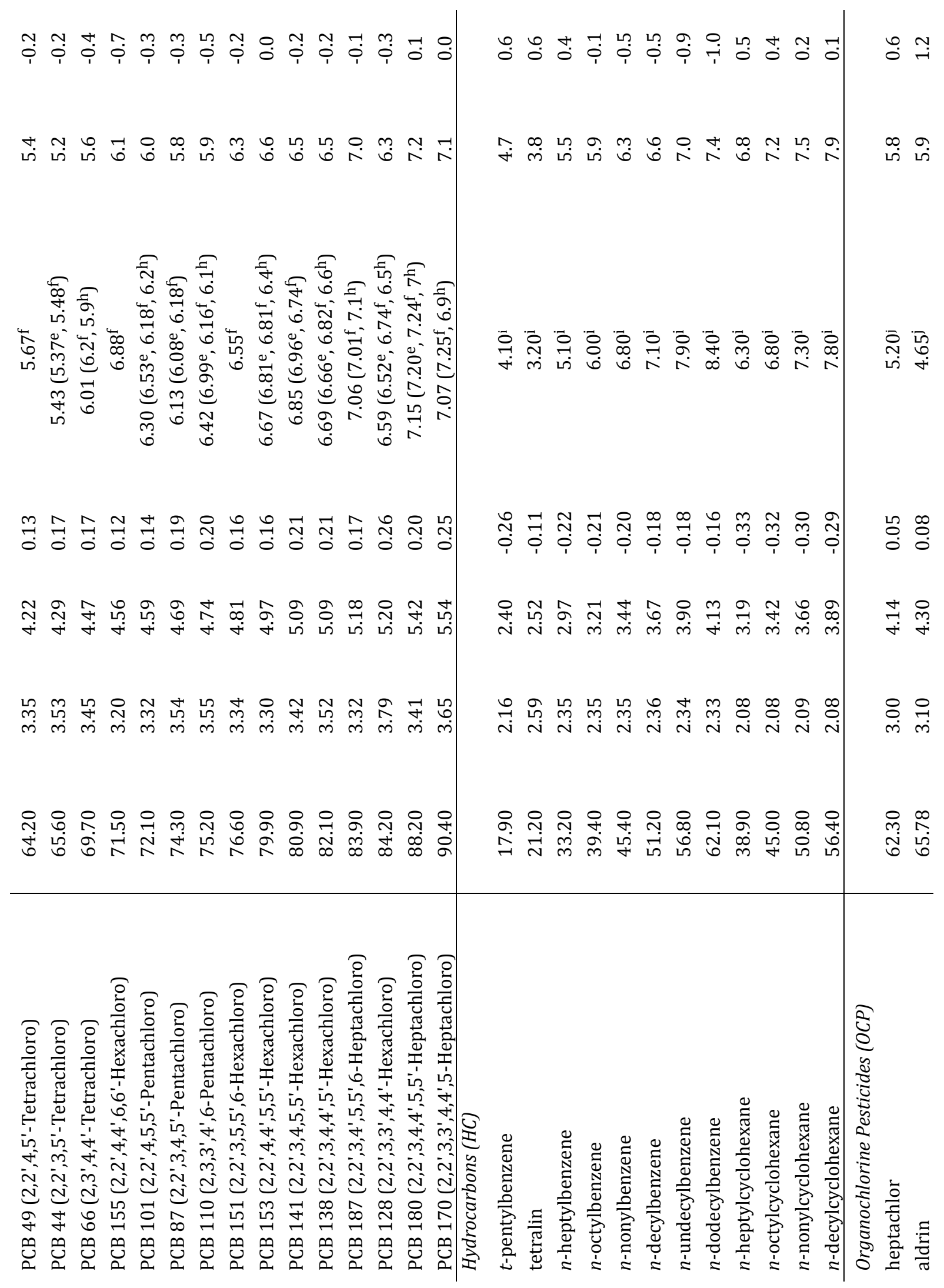




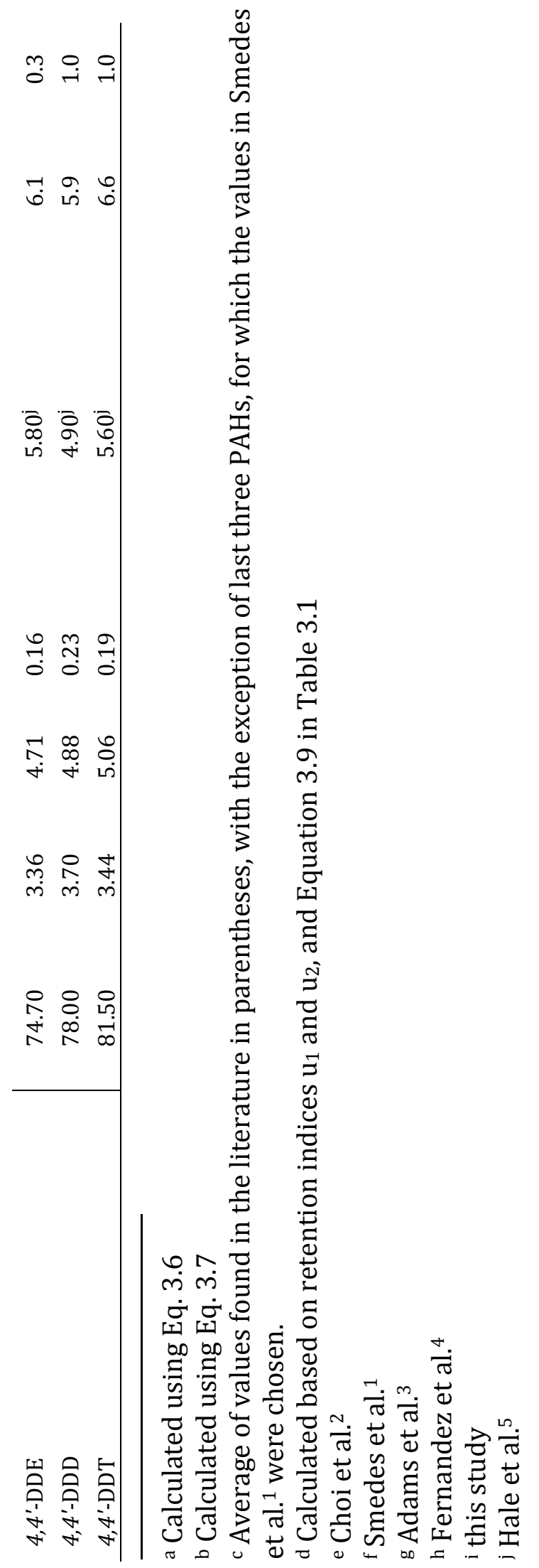




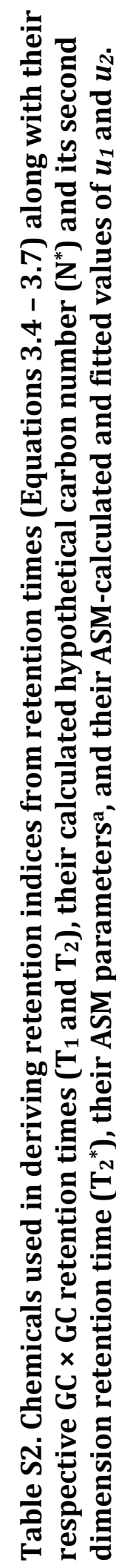

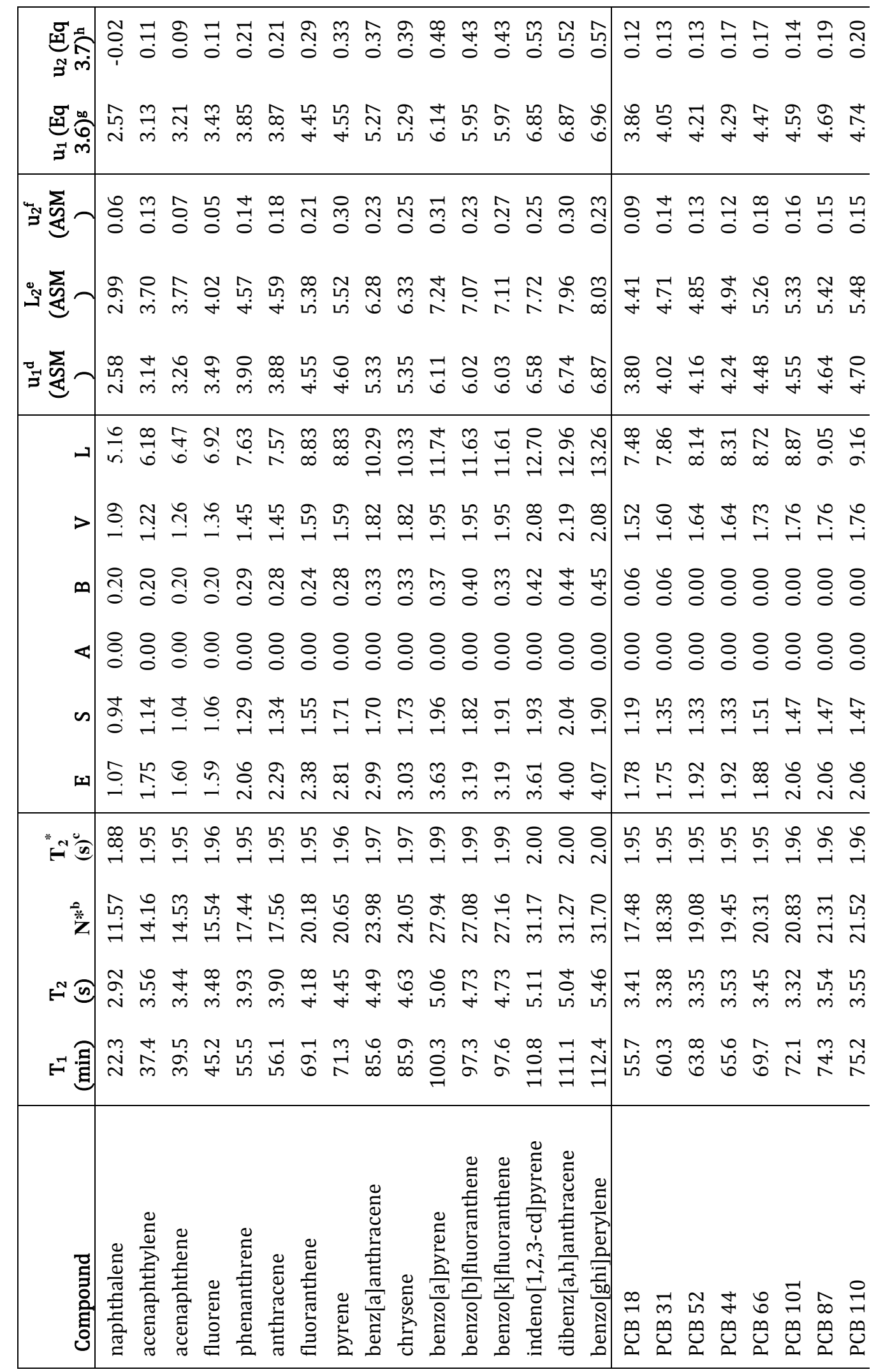




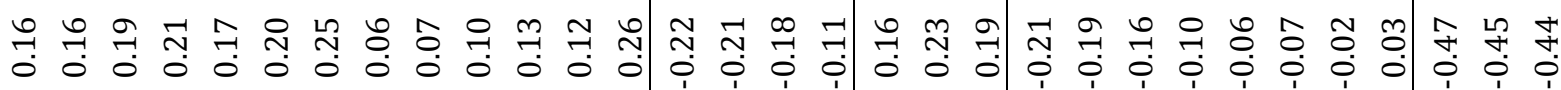

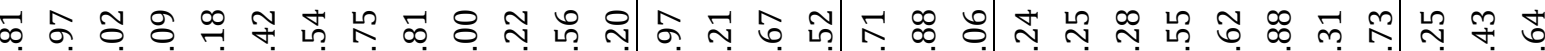

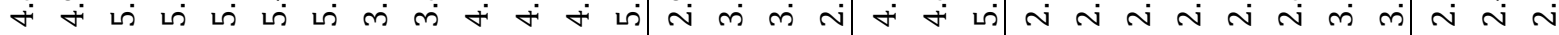

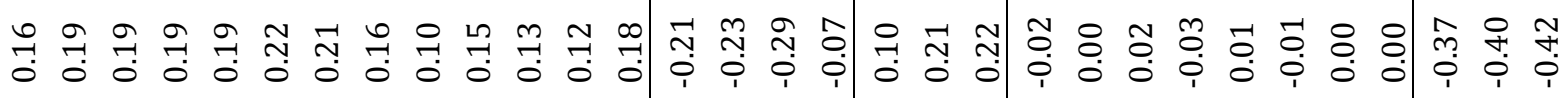

ఫீ

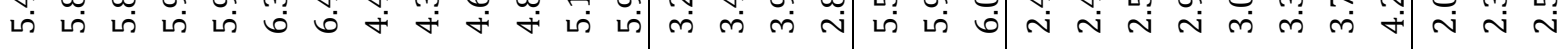

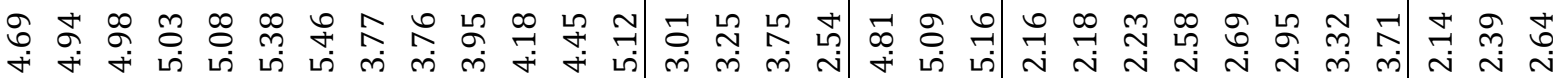

Ұ゙

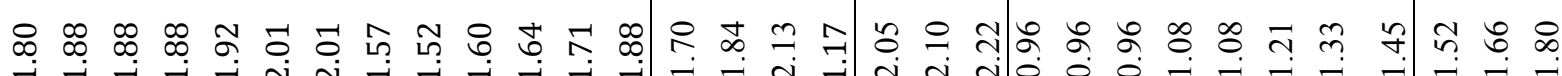

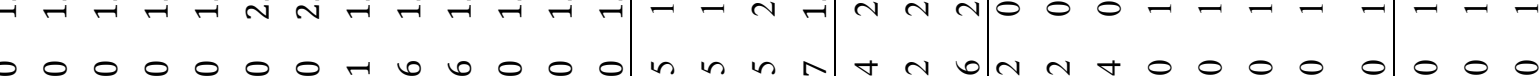

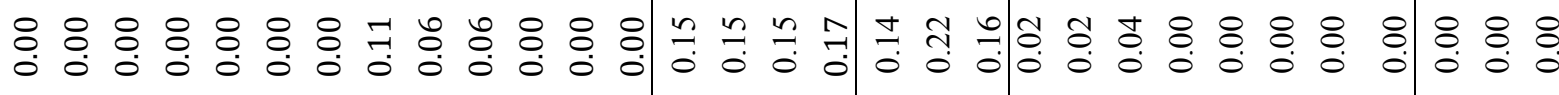

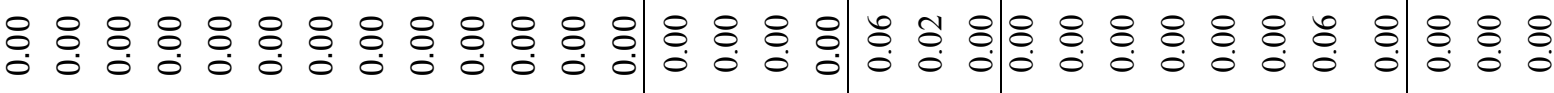

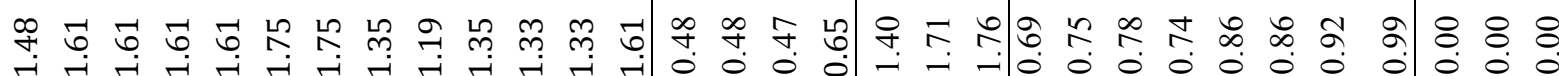

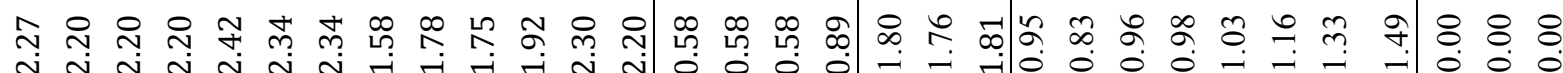

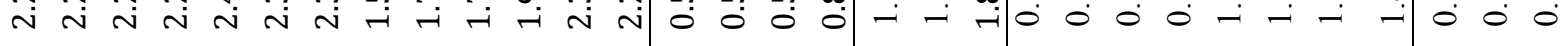
ᄋ œ 0 우

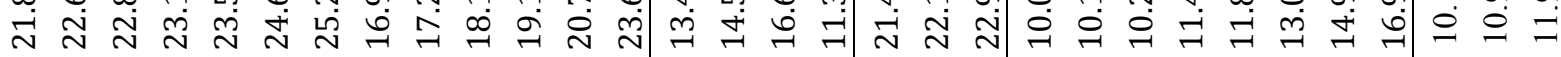

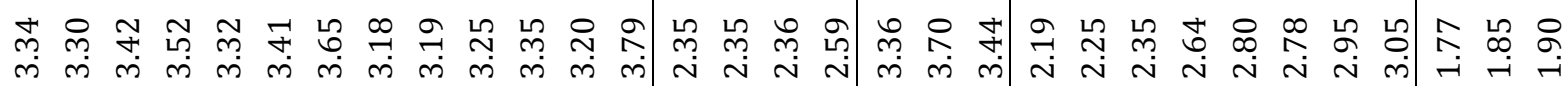

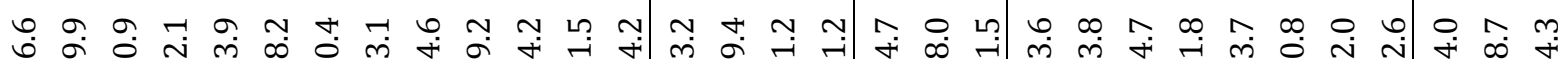

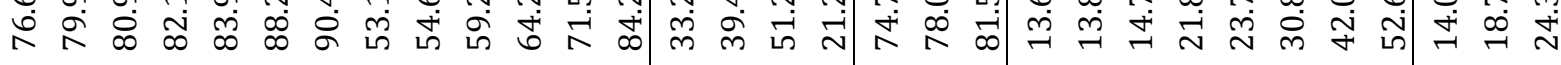

록

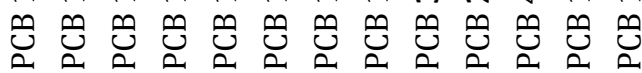

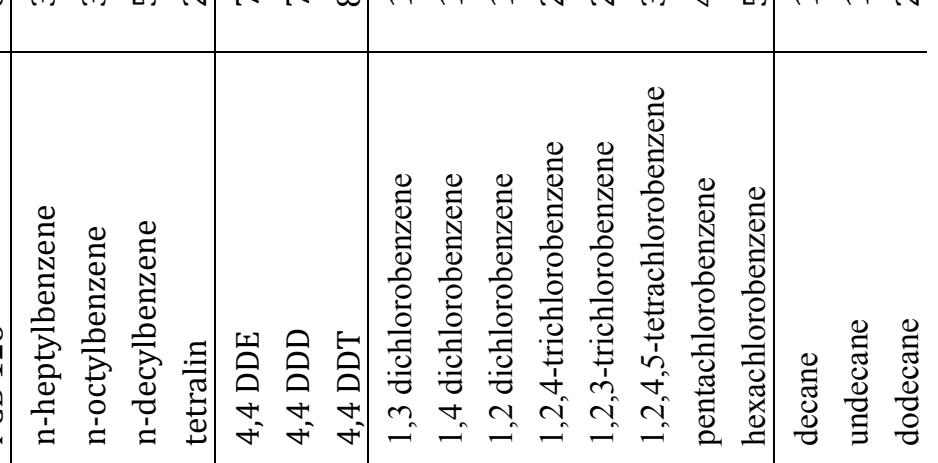




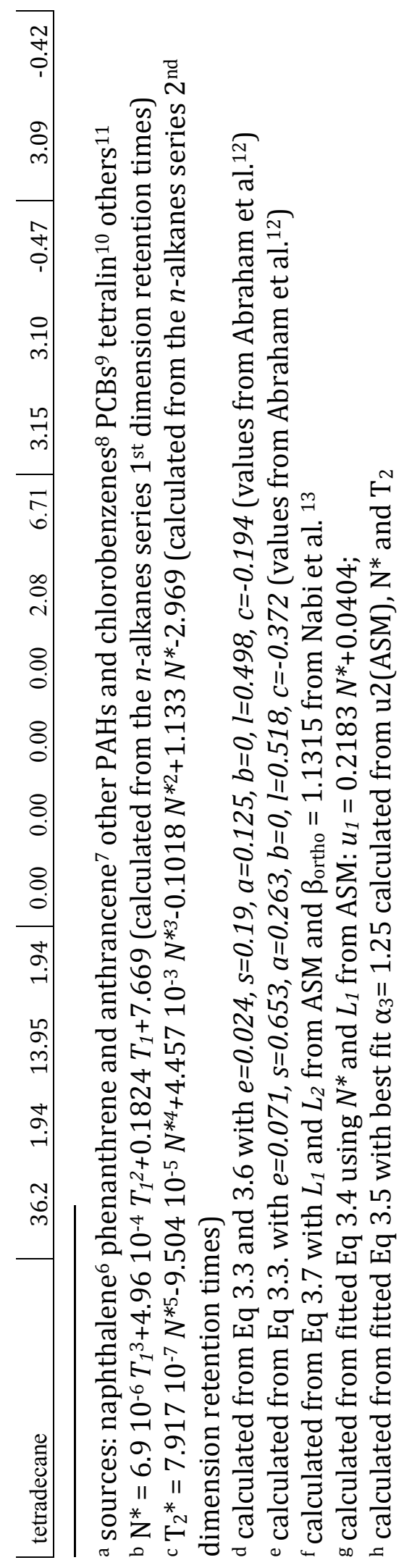




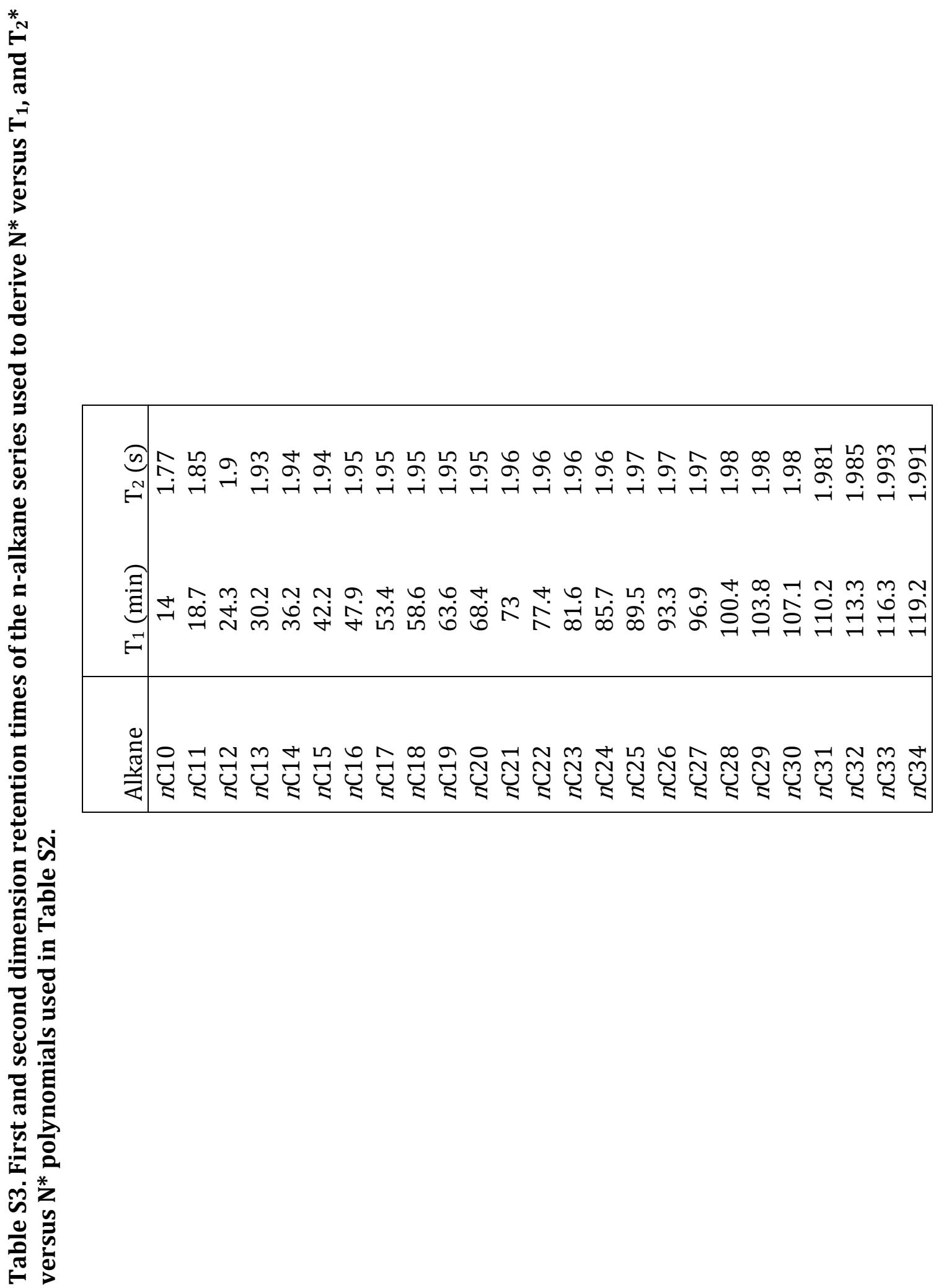




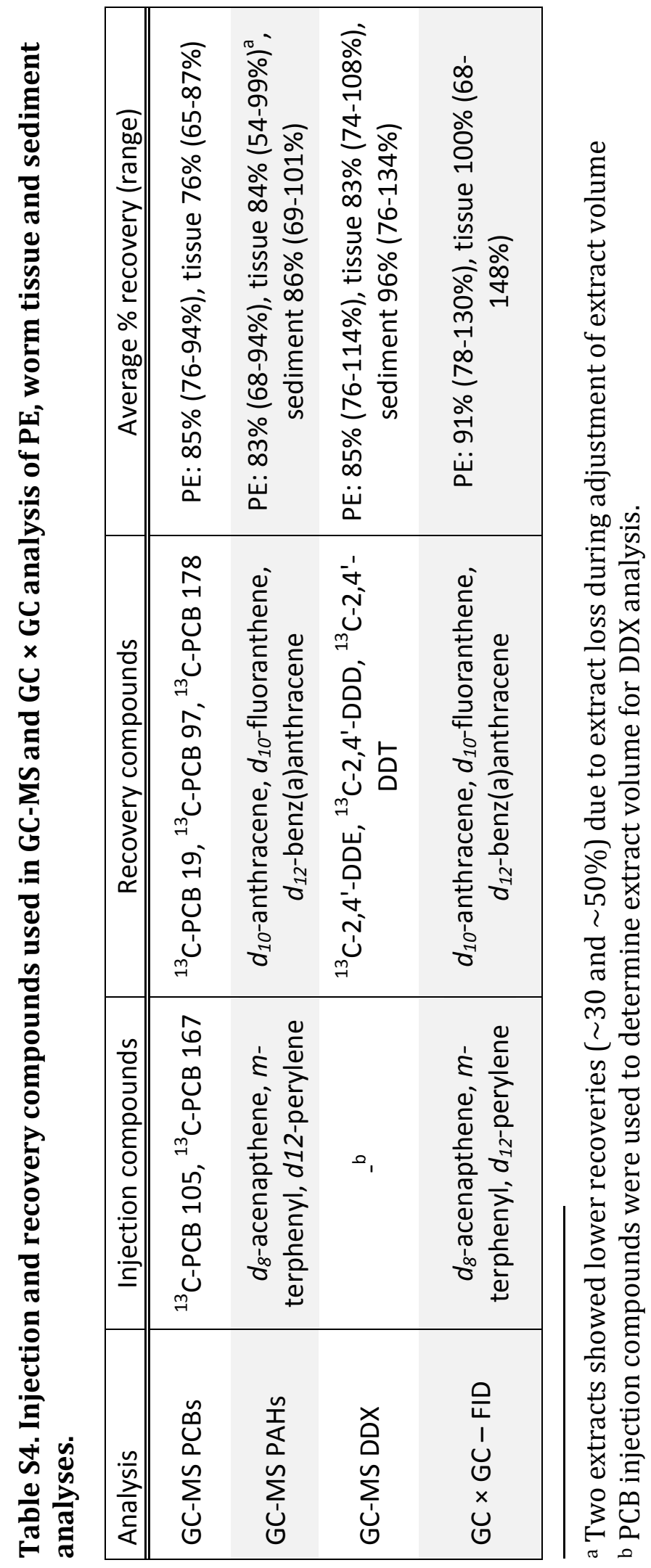


Table S5. Target analytes quantified in the worm tissue, PE samplers and sediments samples.

\begin{tabular}{|c|c|c|c|c|}
\hline Compound & $\begin{array}{l}\text { Quantification } \\
\text { ion }\end{array}$ & $\begin{array}{l}\text { Confirmation } \\
\text { ion }\end{array}$ & $\begin{array}{l}\text { Retention } \\
\text { time (min) }\end{array}$ & $\begin{array}{l}\text { Target } \\
\text { analyte \# }\end{array}$ \\
\hline naphthalene & 128 & 64 & 8.1 & 1 \\
\hline acenaphthylene & 152 & 76 & 12.5 & 2 \\
\hline acenaphthene & 154 & 76 & 13.2 & 3 \\
\hline fluorene & 166 & 82 & 15.3 & 4 \\
\hline phenanthrene & 178 & 89 & 19.9 & 5 \\
\hline anthracene & 178 & 89 & 20.2 & 6 \\
\hline fluoranthene & 202 & 101 & 26.3 & 7 \\
\hline pyrene & 202 & 101 & 27.5 & 8 \\
\hline benz $[a]$ anthracene & 228 & 114 & 34.6 & 9 \\
\hline chrysene & 228 & 114 & 34.8 & 10 \\
\hline benzo[b]fluoranthene & 252 & 126 & 40.7 & 11 \\
\hline benzo[k]fluoranthene & 252 & 126 & 40.9 & 12 \\
\hline benzo[a]pyrene & 252 & 126 & 42.6 & 13 \\
\hline indeno $[1,2,3-c d]$ pyrene & 276 & 138 & 49.5 & 14 \\
\hline $\operatorname{dibenz}[a, h]$ anthracene & 278 & 139 & 51.2 & 15 \\
\hline benzo[ghi]perylene & 276 & 138 & 49.8 & 16 \\
\hline PCB 8 & 222 & 224 & 17.5 & 17 \\
\hline PCB 18 & 256 & 258 & 19.3 & 18 \\
\hline PCB 28 & 256 & 258 & 21.5 & 19 \\
\hline PCB 52 & 292 & 290 & 23.0 & 20 \\
\hline PCB 44 & 292 & 290 & 23.9 & 21 \\
\hline PCB 66 & 292 & 290 & 25.9 & 22 \\
\hline PCB 77 & 292 & 290 & 28.7 & 23 \\
\hline PCB 101 & 326 & 328 & 27.0 & 24 \\
\hline PCB 118 & 326 & 328 & 29.8 & 25 \\
\hline PCB 105 & 326 & 328 & 30.7 & 26 \\
\hline PCB 126 & 326 & 328 & 32.4 & 27 \\
\hline PCB 153 & 360 & 362 & 30.7 & 28 \\
\hline PCB 138 & 360 & 362 & 31.9 & 29 \\
\hline PCB 128 & 360 & 362 & 33.1 & 30 \\
\hline PCB 187 & 396 & 394 & 32.6 & 31 \\
\hline PCB 180 & 396 & 394 & 34.9 & 32 \\
\hline PCB 170 & 396 & 394 & 36.1 & 33 \\
\hline PCB 195 & 430 & 428 & 38.0 & 34 \\
\hline PCB 206 & 464 & 462 & 40.6 & 35 \\
\hline PCB 209 & 498 & 500 & 42.3 & 36 \\
\hline $2,4^{\prime}-\mathrm{DDE}$ & 318 & 320 & 14.8 & 37 \\
\hline 4,4'-DDE & 318 & 320 & 16.1 & 38 \\
\hline 2,4'-DDD & 235 & 237 & 16.4 & 39 \\
\hline 2,4'-DDT & 235 & 237 & 17.5 & 40 \\
\hline $4,4^{\prime}-\mathrm{DDD}^{\mathrm{c}}$ & 235 & 237 & 18.1 & 41 \\
\hline 4,4'-DDT & 235 & 237 & 19.2 & 42 \\
\hline
\end{tabular}

${ }^{c}$ Alternative quantification ions were used when 235/237 pair was too high to integrate: 165 and 199. 
Table S6. Target analyte concentrations in blank worms and in procedure blanks.

\begin{tabular}{|c|c|c|c|c|}
\hline & Procedure $b$ & $\mathrm{~N}=3$ ) & Blank Wor & l=3) \\
\hline & C (ng & & $C_{\text {worm }}(n$ & \\
\hline Compound & Average & SD & Average & SD \\
\hline naphthalene & 51 & 23 & 170 & 100 \\
\hline acenaphthylene & $<5$ & & 17 & 7 \\
\hline acenaphthene & $<5$ & & 96 & 49 \\
\hline fluorene & $<6$ & & 130 & 89 \\
\hline phenanthrene & 146 & 174 & 1400 & 560 \\
\hline anthracene & $<35$ & & 19 & 15 \\
\hline fluoranthene & 18 & 7 & 160 & 130 \\
\hline pyrene & 8 & 2 & 130 & 56 \\
\hline benz $[a]$ anthracene & $<10$ & & 16 & 5 \\
\hline chrysene & $<10$ & & 64 & 50 \\
\hline benzo[b]fluoranthene & $<10$ & & $<9$ & \\
\hline benzo[k]fluoranthene & $<10$ & & $<7$ & \\
\hline benzo[a]pyrene & $<10$ & & $<10$ & \\
\hline indeno[1,2,3-cd]pyrene & $<10$ & & $<13$ & \\
\hline benzo[ghi]perylene & $<10$ & & $<11$ & \\
\hline $\operatorname{dibenz}[a, h]$ anthracene & $<10$ & & $<12$ & \\
\hline PCB 8 & $<2$ & & $<1$ & \\
\hline PCB 18 & $<2$ & & $<2$ & \\
\hline PCB 28/31 & $<2$ & & 7 & 4 \\
\hline PCB 52 & $<3$ & & 7 & 5 \\
\hline PCB 44 & $<3$ & & 4 & 1 \\
\hline PCB 66/80 & $<3$ & & $<2$ & \\
\hline PCB 77 & $<3$ & & 5 & 1 \\
\hline PCB 101/89/90 & $<3$ & & 16 & 9 \\
\hline PCB 118 & $<3$ & & 14 & 20 \\
\hline PCB 105 & $<3$ & & 10 & 6 \\
\hline PCB 126 & $<3$ & & 11 & 14 \\
\hline PCB 153/132 & $<4$ & & 70 & 30 \\
\hline PCB 138 & $<4$ & & 41 & 20 \\
\hline PCB 128 & $<4$ & & 9 & 4 \\
\hline PCB $187 / 182$ & $<4$ & & 20 & 5 \\
\hline PCB 180/193 & $<4$ & & 21 & 9 \\
\hline PCB 170 & $<4$ & & 8 & 2 \\
\hline PCB 195 & $<5$ & & 4 & 1 \\
\hline PCB 206 & $<5$ & & 6 & 2 \\
\hline PCB 209 & $<7$ & & 13 & 8 \\
\hline $2,4^{\prime}-\mathrm{DDE}$ & n.m. ${ }^{e}$ & & $<7$ & \\
\hline $4,4^{\prime}-\mathrm{DDE}$ & n.m. & & $<5$ & \\
\hline 2,4'-DDD & n.m. & & $<2$ & \\
\hline 2,4'-DDT & n.m. & & $<4$ & \\
\hline 4,4'-DDD & n.m. & & $<2$ & \\
\hline 4,4'-DDT & n.m. & & $<4$ & \\
\hline
\end{tabular}

d Concentration calculated assuming $0.04 \mathrm{~g}$ of sample (i.e., $4 \mathrm{~g}$ worm with $\mathrm{f}_{\text {lipid }}$ of 0.01 or $0.04 \mathrm{~g}$ of $\mathrm{PE}$ ). Worm were 3-6 $\mathrm{g}_{\mathrm{w} . w .}$ with $\sim 1 \% \mathrm{f}_{\text {lipid. }}$. Tumbled PE strips were $10-20 \mathrm{mg}$.

e n.m.= not measured. 
Table S7. Concentration of target analytes in 303.1 sediment and in worms and PE incubated with 303.1 sediment

\begin{tabular}{|c|c|c|c|c|}
\hline \multirow[b]{2}{*}{ Compound } & \multicolumn{2}{|c|}{$C_{\text {worm }}\left(\mathrm{ng} / \mathrm{g}_{\text {lipid }}\right)$} & \multirow[t]{2}{*}{$C_{P E}(n g / g)$} & \multirow[t]{2}{*}{$C_{\text {SED }}\left(n g / g_{d w}\right)$} \\
\hline & Average & SD & & \\
\hline naphthalene & 130 & 66 & 54 & 83 \\
\hline acenaphthylene & 38 & 13 & 150 & 13 \\
\hline acenaphthene & 400 & 73 & 9,300 & $<17$ \\
\hline fluorene & 74 & 55 & 4,500 & $<15$ \\
\hline phenanthrene & 420 & 87 & 22,000 & 340 \\
\hline anthracene & 460 & 220 & 17,000 & 600 \\
\hline fluoranthene & 3300 & 540 & 57,000 & 1200 \\
\hline pyrene & 2100 & 240 & 42,000 & 830 \\
\hline benz $[a]$ anthracene & 300 & 96 & 6,000 & 340 \\
\hline chrysene & 420 & 210 & 8,200 & 110 \\
\hline benzo[b]fluoranthene & 130 & 23 & 5,500 & 310 \\
\hline benzo[k]fluoranthene & 140 & 61 & 3,400 & 210 \\
\hline benzo[a]pyrene & 110 & 30 & 3,500 & 250 \\
\hline indeno $[1,2,3-c d]$ pyrene & $<17$ & $<13$ & 2,500 & 240 \\
\hline benzo[ghi]perylene & $<12$ & $<9$ & 1,600 & 240 \\
\hline $\operatorname{dibenz}[a, h]$ anthracene & $<23$ & $<19$ & 23 & 14.0 \\
\hline PCB 8 & $<2$ & & n.m. ${ }^{f}$ & n.m. \\
\hline PCB 18 & 10 & 3 & n.m. & n.m. \\
\hline PCB 28/31 & 11 & 2 & n.m. & n.m. \\
\hline PCB 52 & 57 & 8 & n.m. & n.m. \\
\hline РСB 44 & 7 & 6 & n.m. & n.m. \\
\hline PCB 66/80 & 59 & 13 & n.m. & n.m. \\
\hline PCB 77 & 6 & 7 & n.m. & n.m. \\
\hline PCB 101/89/90 & 39 & 6 & n.m. & n.m. \\
\hline PCB 118 & 420 & 83 & n.m. & n.m. \\
\hline PCB 105 & 22 & 11 & n.m. & n.m. \\
\hline PCB 126 & 46 & 35 & n.m. & n.m. \\
\hline PCB 153/132 & 145 & 37 & n.m. & n.m. \\
\hline PCB 138 & 74 & 13 & n.m. & n.m. \\
\hline PCB 128 & 18 & 8 & n.m. & n.m. \\
\hline PCB 187/182 & 65 & 21 & n.m. & n.m. \\
\hline PCB 180/193 & 53 & 13 & n.m. & n.m. \\
\hline PCB 170 & 30 & 15 & n.m. & n.m. \\
\hline PCB 195 & 8 & 6 & n.m. & n.m. \\
\hline PCB 206 & 19 & 5 & n.m. & n.m. \\
\hline PCB 209 & 14 & 6 & n.m. & n.m. \\
\hline 2,4'-DDE & $<3$ & & $<2$ & $<1$ \\
\hline 4,4'-DDE & $<2$ & & 70 & $<1$ \\
\hline 2,4'-DDD & 40 & 8 & $<1$ & $<1$ \\
\hline 2,4'-DDT & $<1$ & & $<2$ & $<1$ \\
\hline $4,4^{\prime}-\mathrm{DDD}$ & 100 & 24 & $<1$ & 36 \\
\hline 4,4'-DDT & $<1$ & & $<3$ & 150 \\
\hline
\end{tabular}

${ }^{f}$ n.m. not measured 
Table S8. Concentration of target analytes in C400 sediment and in worms and PE incubated with $\mathrm{C400}$ sediment.

\begin{tabular}{|c|c|c|c|c|}
\hline & \multicolumn{2}{|c|}{$\mathrm{C}_{\text {worm }}\left(\mathrm{ng} / \mathrm{g}_{\text {lipid }}\right)$} & \multirow[t]{2}{*}{$C_{P E}(\mathrm{ng} / \mathrm{g})$} & \multirow[t]{2}{*}{$\mathrm{C}_{\mathrm{SED}}\left(\mathrm{ng} / \mathrm{g}_{\mathrm{dw}}\right)$} \\
\hline Compound & Average & SD & & \\
\hline naphthalene & 130 & 46 & 90 & 260 \\
\hline acenaphthylene & 390 & 150 & 7,400 & 1,200 \\
\hline acenaphthene & 130 & 33 & 1,600 & 58 \\
\hline fluorene & 92 & 34 & 1,900 & 220 \\
\hline phenanthrene & 480 & 210 & 15,000 & 1,300 \\
\hline anthracene & 5,700 & 2,200 & 52,000 & 5,200 \\
\hline fluoranthene & 30,000 & 15,000 & 490,000 & 12,000 \\
\hline pyrene & 14,000 & 7,700 & 330,000 & 7,100 \\
\hline benz[a]anthracene & 2,900 & 1,300 & 83,000 & 3,400 \\
\hline chrysene & 2,300 & 940 & 37,000 & 5,900 \\
\hline benzo[b]fluoranthene & 3,300 & 2,000 & 95,000 & 5,100 \\
\hline benzo[k]fluoranthene & 2,000 & 1,100 & 39,000 & 2,700 \\
\hline benzo[a]pyrene & 3,300 & 2,900 & 61,000 & 3,500 \\
\hline indeno[1,2,3-cd]pyrene & 230 & 150 & 16,000 & 1,900 \\
\hline benzo[ghi]perylene & 240 & 140 & 7,600 & 1,400 \\
\hline $\operatorname{dibenz}[a, h]$ anthracene & 140 & 47 & 1,500 & 490 \\
\hline PCB 8 & $<2$ & & $<2$ & n.m. ${ }^{\mathrm{g}}$ \\
\hline PCB 18 & 15 & 3 & 18 & n.m. \\
\hline PCB 28/31 & 28 & 9 & 28 & n.m. \\
\hline PCB 52 & 210 & 14 & 140 & n.m. \\
\hline PCB 44 & 22 & 2 & 51 & n.m. \\
\hline PCB 66/80 & 55 & 12 & 84 & n.m. \\
\hline PCB 77 & 9 & 5 & 9 & n.m. \\
\hline PCB 101/89/90 & 180 & 12 & 240 & n.m. \\
\hline PCB 118 & 170 & 82 & 220 & n.m. \\
\hline PCB 105 & 61 & 4 & 62 & n.m. \\
\hline PCB 126 & $<4$ & & $<3$ & n.m. \\
\hline PCB 153/132 & 210 & 21 & 270 & n.m. \\
\hline PCB 138 & 150 & 4 & 190 & n.m. \\
\hline PCB 128 & 30 & 6 & 38 & n.m. \\
\hline PCB 187/182 & 65 & 5 & 65 & n.m. \\
\hline PCB 180/193 & 74 & 9 & 110 & n.m. \\
\hline PCB 170 & 32 & 11 & 49 & n.m. \\
\hline PCB 195 & 8 & 1 & 6 & n.m. \\
\hline PCB 206 & 22 & 8 & $<3$ & n.m. \\
\hline PCB 209 & 12 & 4 & $<3$ & n.m. \\
\hline 2,4'-DDE & $<11$ & & 69 & $<2$ \\
\hline 4,4'-DDE & 420 & 230 & 1800 & 160 \\
\hline $2,4^{\prime}-\mathrm{DDD}$ & 2600 & 330 & 1000 & 110 \\
\hline 2,4'-DDT & $<5$ & & $<5$ & $<1$ \\
\hline 4,4'-DDD & 11000 & 2800 & 3100 & 450 \\
\hline $4,4^{\prime}$-DDT & 230 & 200 & $<8$ & 290 \\
\hline
\end{tabular}

g n.m. not measured 
Table S9. Concentration of target analytes in $\mathrm{C1800}$ sediment and in worms and PE incubated with C1800 sediment

\begin{tabular}{|c|c|c|c|c|}
\hline & \multicolumn{2}{|c|}{$C_{\text {worm }}\left(\mathrm{ng} / \mathrm{g}_{\text {lipid }}\right)$} & \multirow[t]{2}{*}{$C_{P E}(\mathrm{ng} / \mathrm{g})$} & \multirow[t]{2}{*}{$C_{\text {SED }}\left(n g / g_{d w}\right)$} \\
\hline Compound & Average & SD & & \\
\hline naphthalene & 180 & 59 & 74 & 450 \\
\hline acenaphthylene & 55 & 48 & 540 & 290 \\
\hline acenaphthene & 76 & 30 & 130 & 87 \\
\hline fluorene & 46 & 24 & $<58$ & 170 \\
\hline phenanthrene & 160 & 52 & 460 & 1,200 \\
\hline anthracene & 580 & 260 & 3,600 & 2,100 \\
\hline fluoranthene & 2,000 & 1,200 & 4,400 & 2,100 \\
\hline pyrene & 5,100 & 2,400 & 18,000 & 3,900 \\
\hline benz[a]anthracene & 240 & 110 & 1,000 & 1,800 \\
\hline chrysene & 350 & 130 & 1,800 & 2,500 \\
\hline benzo[b]fluoranthene & 1,000 & 470 & 17,000 & 4,700 \\
\hline benzo[k]fluoranthene & 650 & 250 & 11,000 & 1,600 \\
\hline benzo[a]pyrene & 750 & 190 & 9,300 & 3,800 \\
\hline indeno[1,2,3-cd]pyrene & 110 & 58 & 9,000 & 1,900 \\
\hline benzo[ghi]perylene & 140 & 69 & 5,200 & 2,600 \\
\hline $\operatorname{dibenz}[a, h]$ anthracene & 85 & 23 & 650 & 1,100 \\
\hline PCB 8 & $<3$ & & 40 & n.m \\
\hline PCB 18 & 640 & 220 & 170 & n.m \\
\hline PCB 28/31 & 1,000 & 320 & 630 & n.m \\
\hline PCB 52 & 1,300 & 300 & 360 & n.m \\
\hline PCB 44 & 280 & 91 & 230 & n.m \\
\hline PCB 66/80 & 390 & 76 & 260 & n.m \\
\hline PCB 77 & 59 & 16 & 16 & n.m \\
\hline PCB 101/89/90 & 560 & 130 & 400 & n.m \\
\hline PCB 118 & 340 & 71 & 350 & n.m \\
\hline PCB 105 & 230 & 59 & 130 & n.m \\
\hline PCB 126 & 10 & 5.2 & 1.8 & n.m. \\
\hline PCB 153/132 & 330 & 74 & 340 & n.m \\
\hline PCB 138 & 260 & 53 & 300 & n.m \\
\hline PCB 128 & 68 & 18 & 64 & n.m \\
\hline PCB 187/182 & 81 & 32 & 74 & n.m \\
\hline PCB 180/193 & 95 & 38 & 150 & n.m \\
\hline PCB 170 & 57 & 21 & 67 & n.m \\
\hline PCB 195 & $<7$ & & 22 & n.m \\
\hline PCB 206 & 26 & 7 & 18 & n.m \\
\hline PCB 209 & $<9$ & & $<2$ & n.m \\
\hline 2,4'-DDE & $<8$ & & 140 & $<1$ \\
\hline $4,4^{\prime}-\mathrm{DDE}$ & 1200 & 200 & 2400 & 640 \\
\hline 2,4'-DDD & 14000 & 2000 & 2900 & 1400 \\
\hline 2,4'-DDT & $<4$ & & $<3$ & 150 \\
\hline 4,4'-DDD & 72000 & 7000 & 8300 & 5300 \\
\hline $4,4^{\prime}$-DDT & 390 & 80 & $<5$ & 1100 \\
\hline
\end{tabular}


Table S10 Concentration of target analytes in Island End sediment and in worms and PE incubated with Island End sediment.

\begin{tabular}{|c|c|c|c|c|}
\hline & \multicolumn{2}{|c|}{$\mathrm{C}_{\text {worm }}\left(\mathrm{ng} / \mathrm{g}_{\text {lipid }}\right)$} & \multirow[t]{2}{*}{$C_{P E}(n g / g)$} & \multirow[t]{2}{*}{$C_{S E D}\left(n g / g_{d w}\right)$} \\
\hline Compound & Average & SD & & \\
\hline naphthalene & 210 & 20 & 2,100 & 2,900 \\
\hline acenaphthylene & 200 & 130 & 44,000 & 5,400 \\
\hline acenaphthene & 150 & 67 & 4,900 & 730 \\
\hline fluorene & 130 & 14 & 2,800 & 1,600 \\
\hline phenanthrene & 240 & 78 & 37,000 & 8,000 \\
\hline anthracene & 2,500 & 1,300 & 350,000 & 18,000 \\
\hline fluoranthene & 47,000 & 19,000 & $1,600,000$ & 58,000 \\
\hline pyrene & 16,000 & 7,700 & $1,900,000$ & 46,000 \\
\hline benz $[a]$ anthracene & 3,400 & 1,300 & 550,000 & 25,000 \\
\hline chrysene & 4,400 & 1,200 & 380,000 & 16,000 \\
\hline benzo[b]fluoranthene & 4,800 & 1,800 & $1,000,000$ & 33,000 \\
\hline benzo[k]fluoranthene & 2,300 & 450 & 420,000 & 12,000 \\
\hline benzo[a]pyrene & 4,800 & 2,400 & $1,100,000$ & 28,000 \\
\hline indeno[1,2,3-cd]pyrene & 680 & 150 & 200,000 & 18,000 \\
\hline benzo[ghi]perylene & 830 & 640 & 130,000 & 14,000 \\
\hline $\operatorname{dibenz}[a, h]$ anthracene & 770 & 370 & 19,000 & 18,000 \\
\hline PCB 8 & $<2$ & & $<1$ & n.m \\
\hline PCB 18 & 14 & 9 & 5 & n.m \\
\hline PCB 28/31 & 23 & 5 & 25 & n.m \\
\hline PCB 52 & 89 & 19 & 110 & n.m \\
\hline PCB 44 & 19 & 8 & 44 & n.m \\
\hline PCB 66/80 & 37 & 31 & 34 & n.m \\
\hline PCB 77 & 9 & 5 & 3 & n.m \\
\hline PCB 101/89/90 & 150 & 38 & 150 & n.m \\
\hline PCB 118 & 120 & 50 & 130 & n.m \\
\hline PCB 105 & 60 & 4 & 52 & n.m \\
\hline PCB 126 & $<5$ & & & n.m. \\
\hline PCB 153/132 & 380 & 65 & 390 & n.m \\
\hline PCB 138 & 220 & 33 & 170 & n.m \\
\hline PCB 128 & 40 & 12 & 36 & n.m \\
\hline PCB 187/182 & 120 & 27 & 170 & n.m \\
\hline PCB 180/193 & 180 & 43 & 370 & n.m \\
\hline РCB 170 & 83 & 23 & 130 & n.m \\
\hline PCB 195 & $<7$ & & 29 & n.m \\
\hline PCB 206 & $<16$ & & 43 & n.m \\
\hline PCB 209 & $<10$ & & 8 & n.m \\
\hline 2,4'-DDE & n.m. & & $<3$ & $<2$ \\
\hline 4,4'-DDE & n.m. & & $<2$ & $<1$ \\
\hline 2,4'-DDD & n.m. & & $<1$ & $<1$ \\
\hline 2,4'-DDT & n.m. & & $<2$ & $<3$ \\
\hline 4,4'-DDD & n.m. & & $<1$ & $<1$ \\
\hline 4,4'-DDT & n.m. & & $<2$ & $<5$ \\
\hline
\end{tabular}




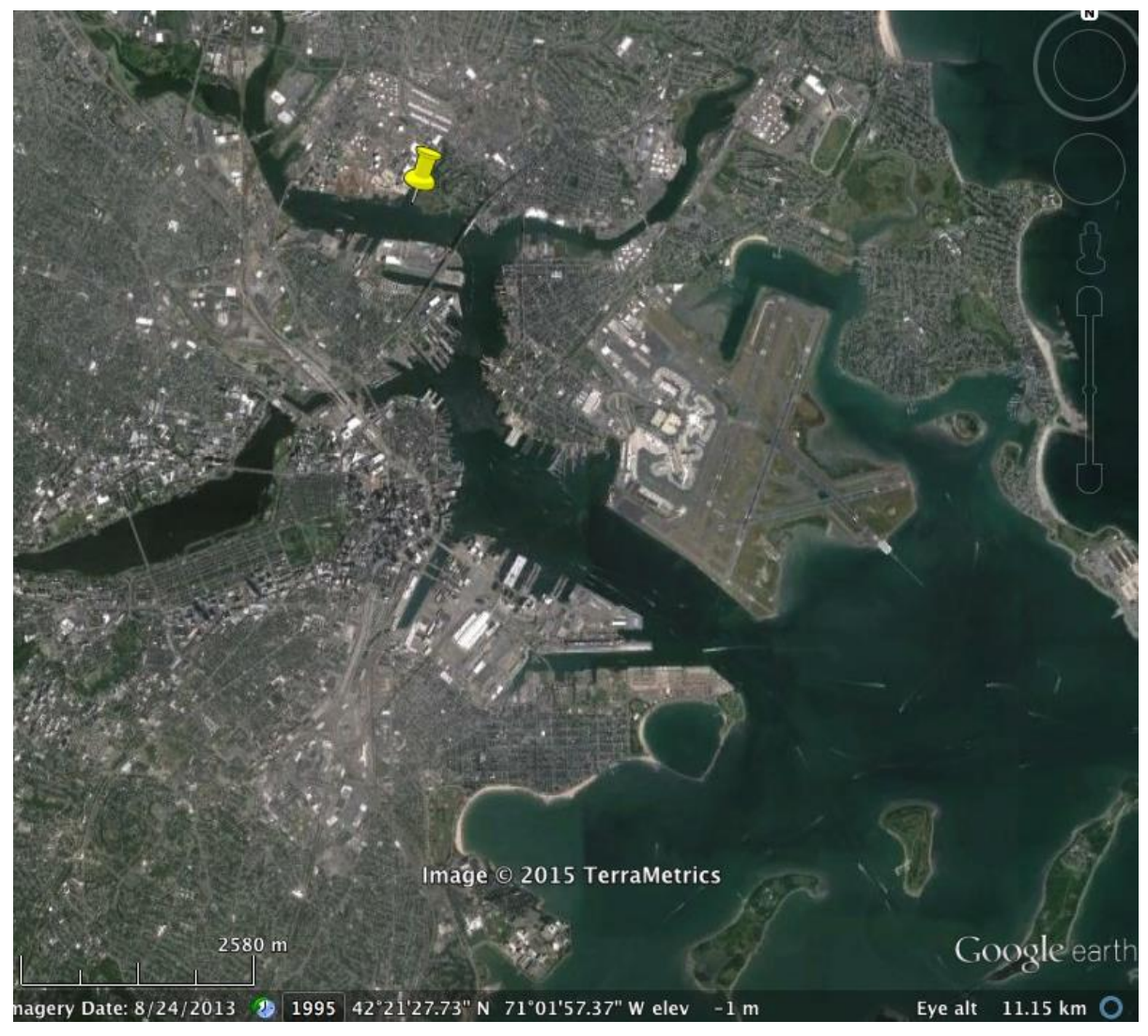

Figure S1. Location (yellow pin) of Island End, Chelsea MA, sampling site within Inner Boston Harbor. 


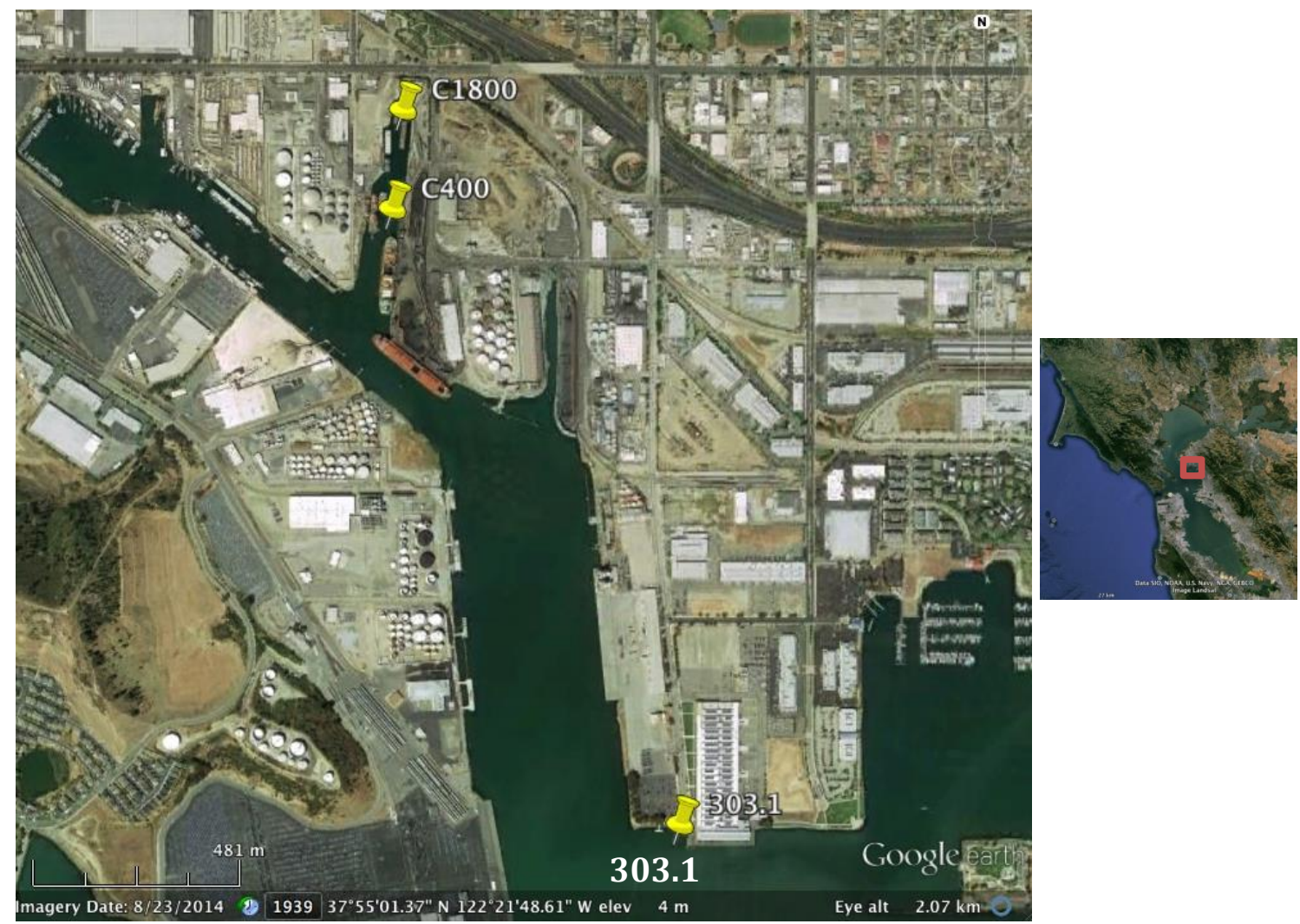

Figure S2. Map of sampling locations in Lauritzen Channel, Richmond,CA (C1800 and C400) and background site 303.1. Red square in inset shows approximate location of Lauritzen Channel in San Francisco Bay, CA. 


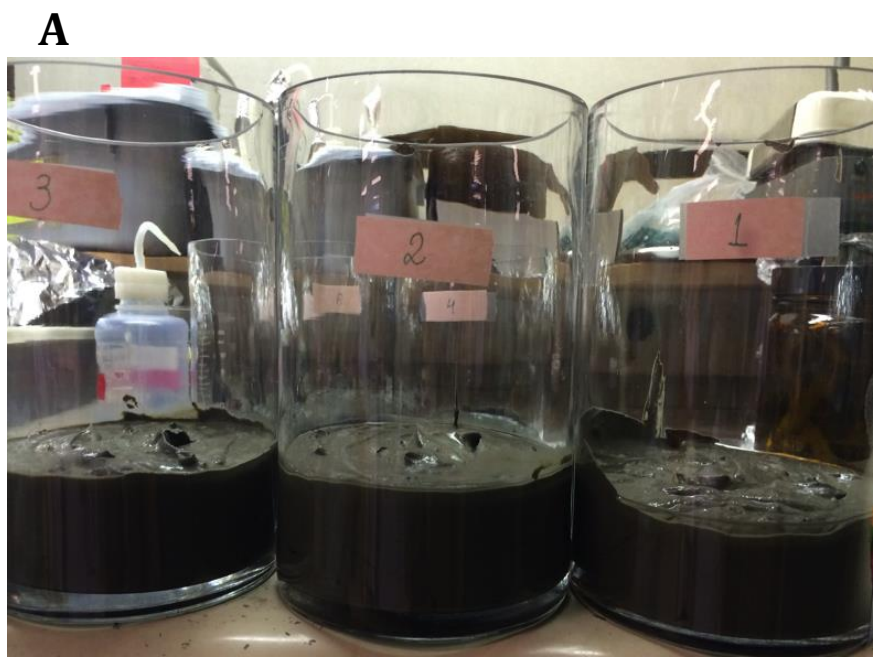

C
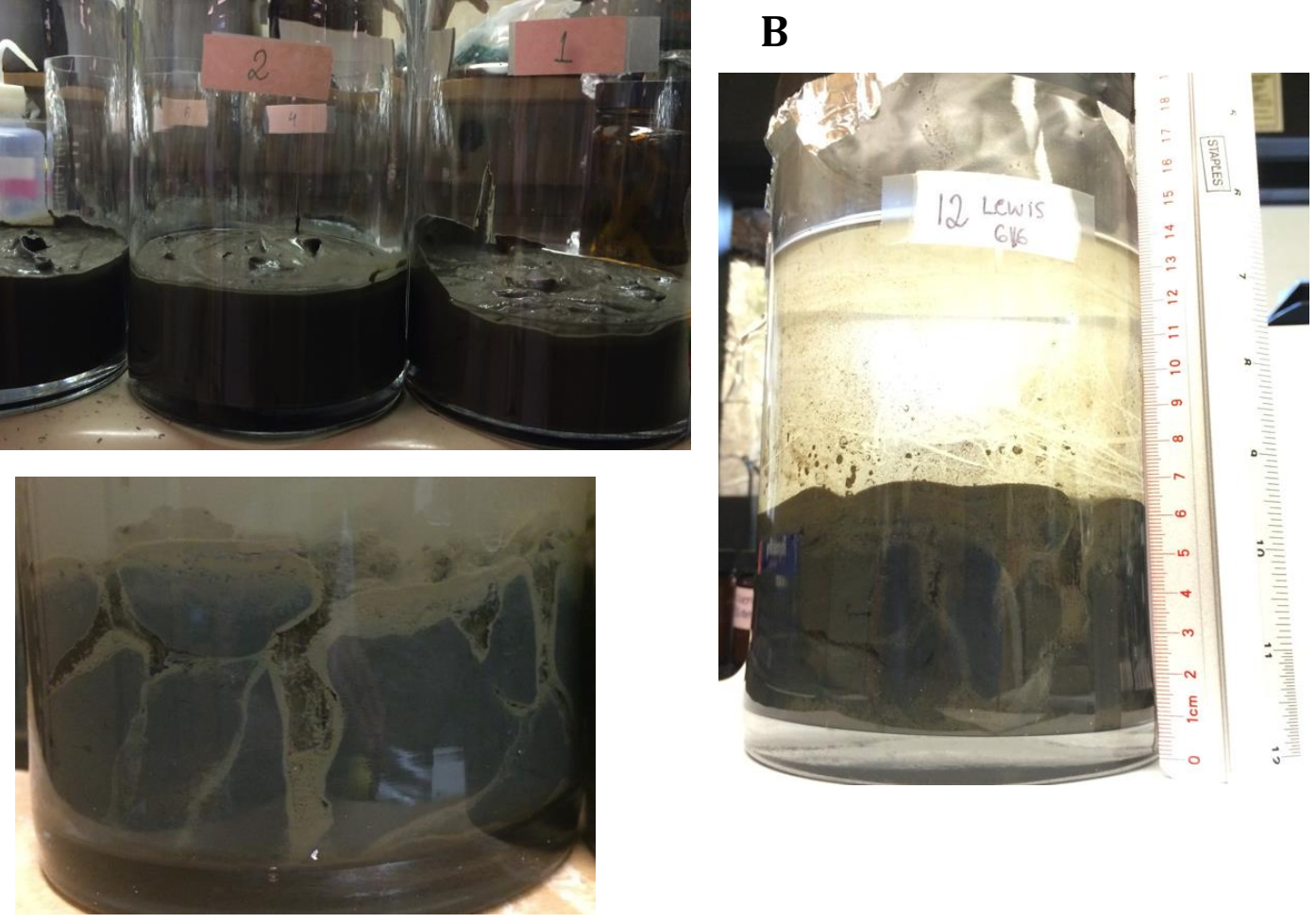

Figure S3. Experimental setup for bioaccumulation experiments: A. Aquaria loaded with sediment before addition of water and worms. B. Aquaria with water, Island End sediment and worm after the 28 day incubation. Multiple borrows are visible and the surface of the sediment is no longer flat due to the worm activity. C. Close-up of burrows observed after 28 days in $\mathrm{C} 400$ sediment. 


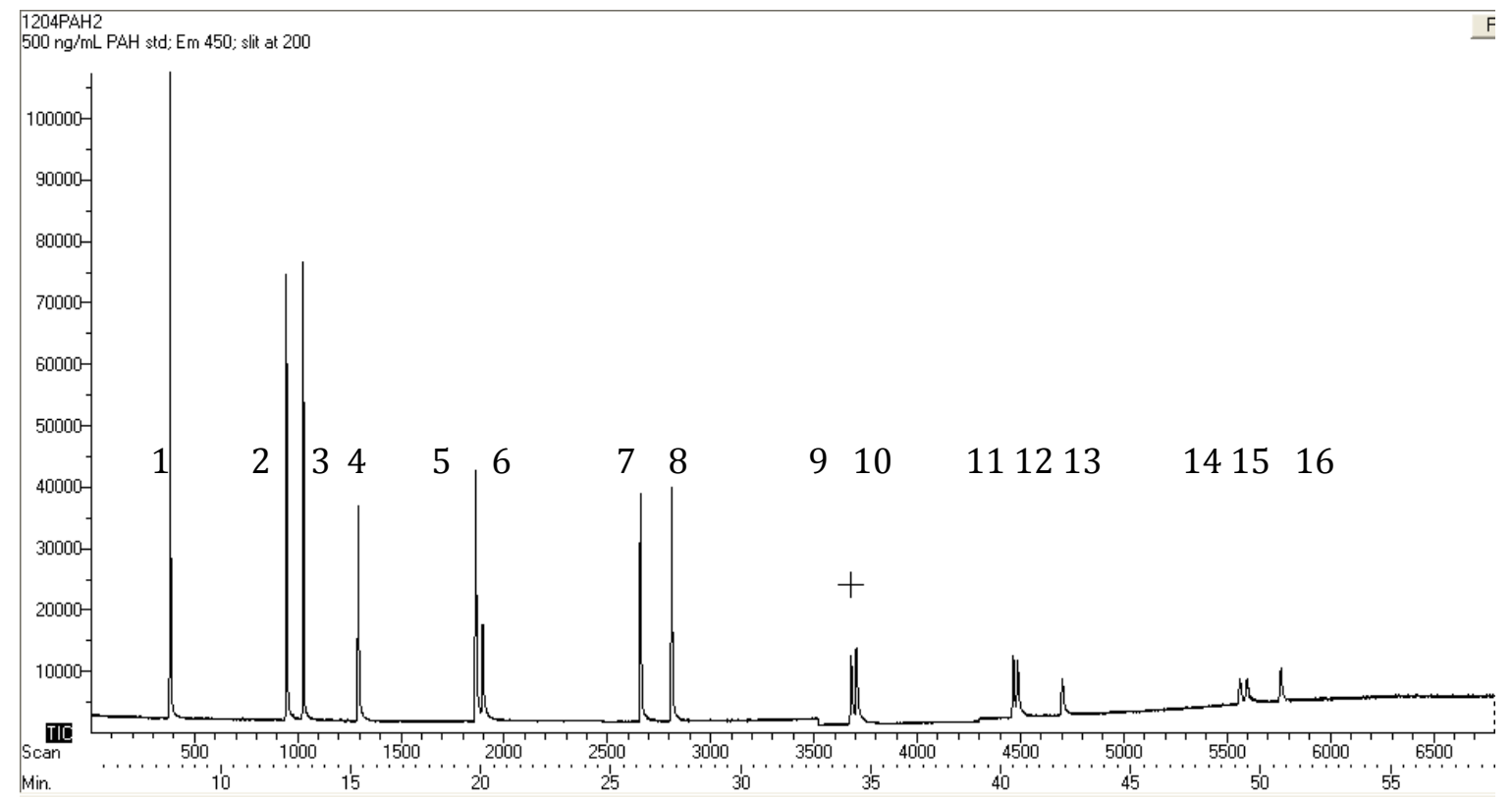

Figure S4. Chromatogram of a $500 \mathrm{ng} / \mathrm{mL}$ dilution of US 106N PAH mixture on a $60 \mathrm{~m}$ DB5 column using $1 \mu \mathrm{L}$ split/splitless injections (see also Section 3.2.9). Analytes are as follows naphthalene (1), acenaphthylene (2), acenaphthene (3), fluorene (4), phenanthrene (5), anthracene (6), fluoranthene (7), pyrene (8), benz[a] anthracene (9), chrysene (10), benzo[ $b]$ fluoranthene (11), benzo[k]fluoranthene (12), benzo[ $a]$ pyrene (13), indeno[1,2,3$c d]$ pyrene (14), dibenz $[a, h]$ anthracene(15), benzo[ghi]perylene (16). Analyte numbers are the same as in Table S2, which also lists retention times and quantification ions. 


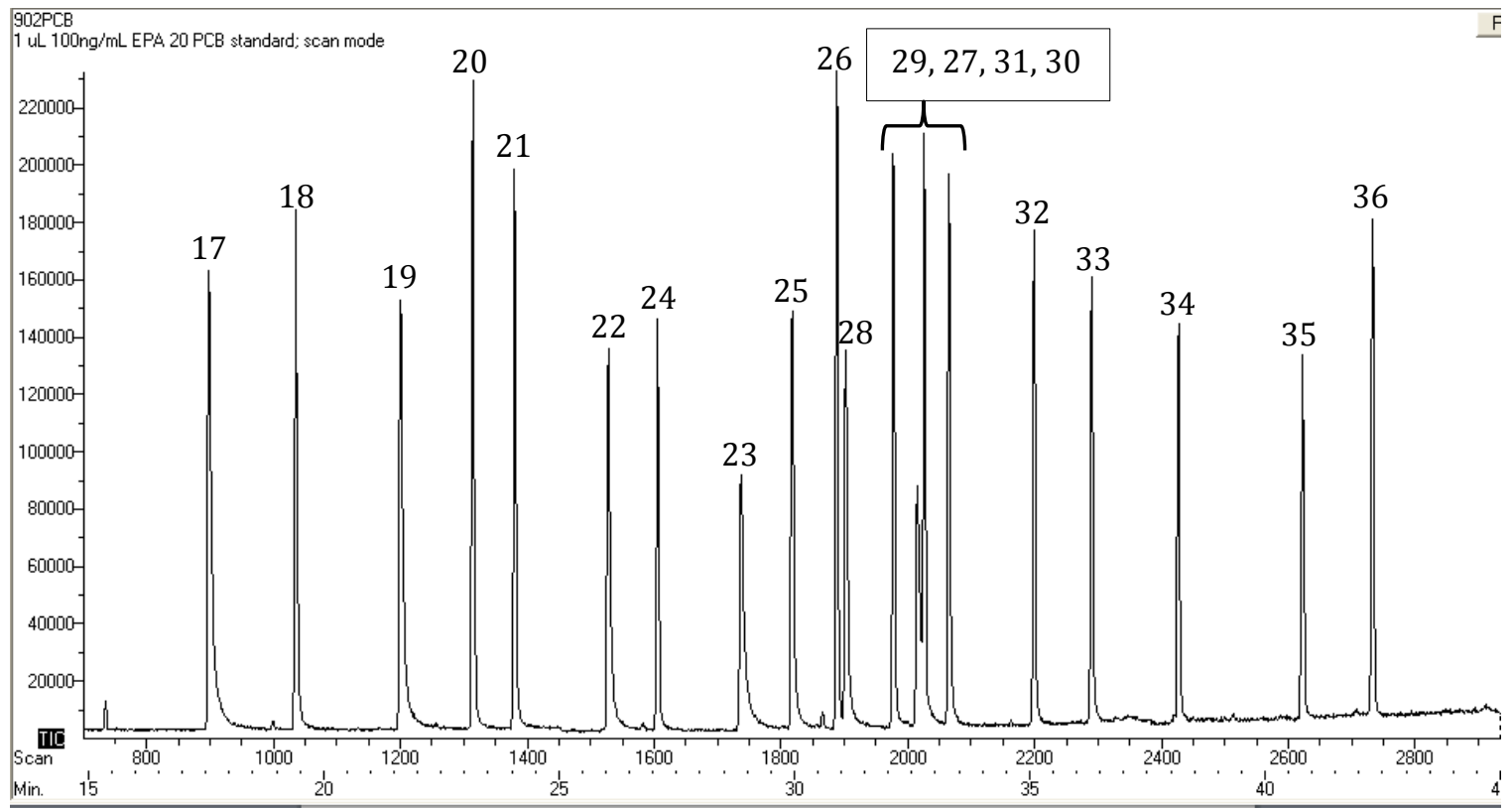

Figure S5. Chromatogram of a $100 \mathrm{ng} / \mathrm{mL}$ dilution of EPA 20 PCB mixture on a $60 \mathrm{~m} \mathrm{DB}-5$ column using $1 \mu \mathrm{L}$ split/splitless injections (see also Section 3.2.9). Numbers refer to analytes in Table S2. Analytes with number assignment in parentheses are as follows, grouped by number of chlorine atoms, dichlorobiphenyls: PCB 8 (17), trichlorobiphenyls: PCB 18 (18), PCB 28 (19), tetraclorobiphenyls: PCB 52 (20), PCB 44 (21), PCB 66 (22), PCB 77 (23), pentachlorobiphenyls: PCB 101 (24), PCB 118 (25), PCB 105 (26), PCB 126 (27), hexachlorobiphenyls: PCB 153 (28), PCB 138 (29), PCB 128 (30), heptachlorobiphenyls: PCB 187 (31), PCB 180 (32), PCB 170 (33), octachlorobiphenyls: PCB 195 (34), nonachlorobiphenyls: PCB 206 (35), decachlorobiphenyls: PCB 209 (36). Analyte numbers are the same as in Table S2, which also lists retention times and quantification ions. 


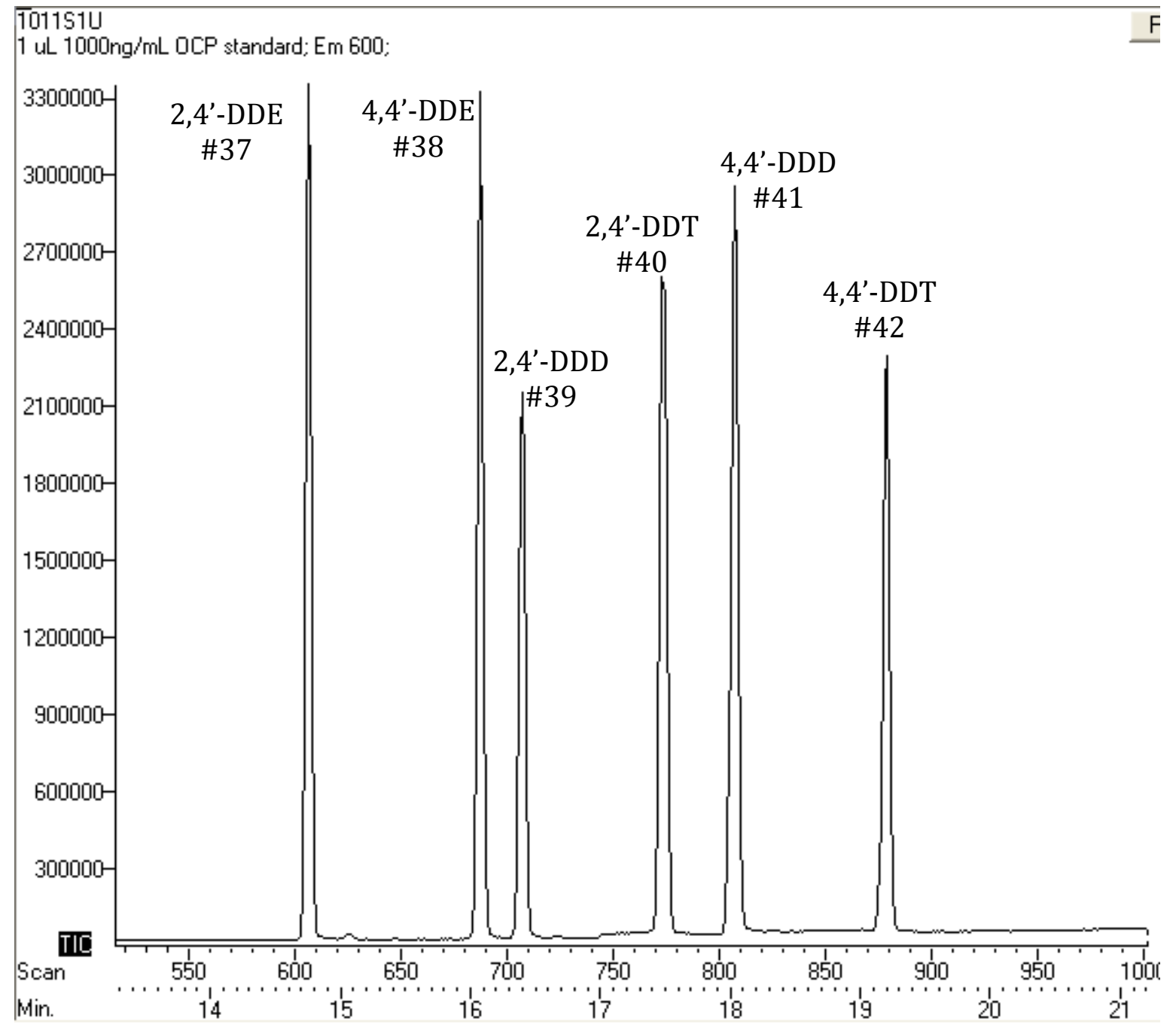

Figure S6. Chromatogram of a $1000 \mathrm{ng} / \mathrm{mL}$ DDx standard mixture on a $30 \mathrm{~m}$ DB-XLB column (see also Section 3.2.9). Numbers refer to analytes in Table S2, which also lists quantification ions and retention times. 

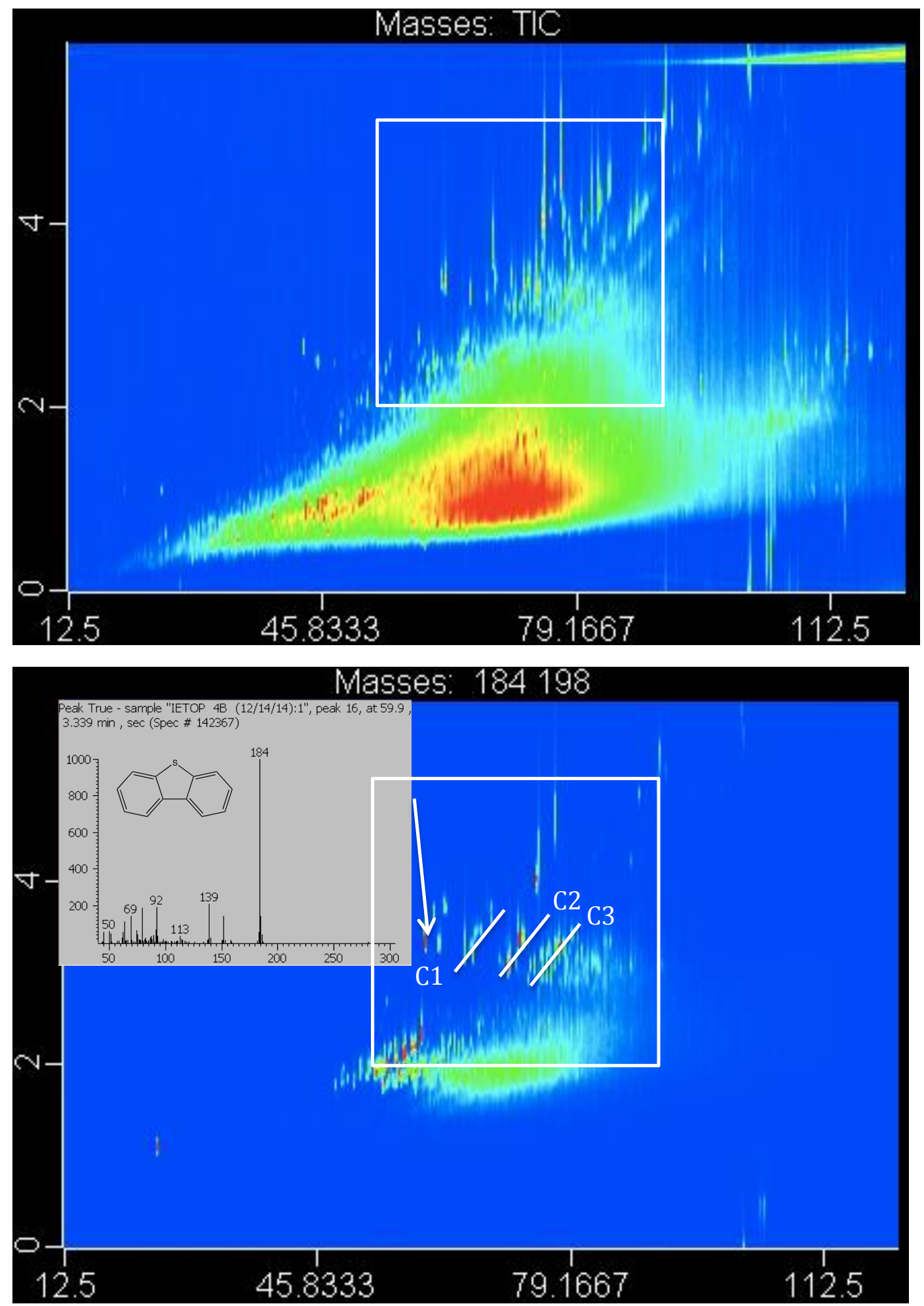

Figure S7. GC $\times$ GC-TOFMS total ion chromatogram of PE extract incubated with Island End sediment (top) and reconstructed ion chromatogram for dibenzothiophene ( $\mathrm{m} / \mathrm{z}$ of 184 ) 
and C1-dibenzothiophene (m/z of 198). Also visible on the bottom panel are the C2 and C3 dibenzothiophene series. White box is for visual guidance only.
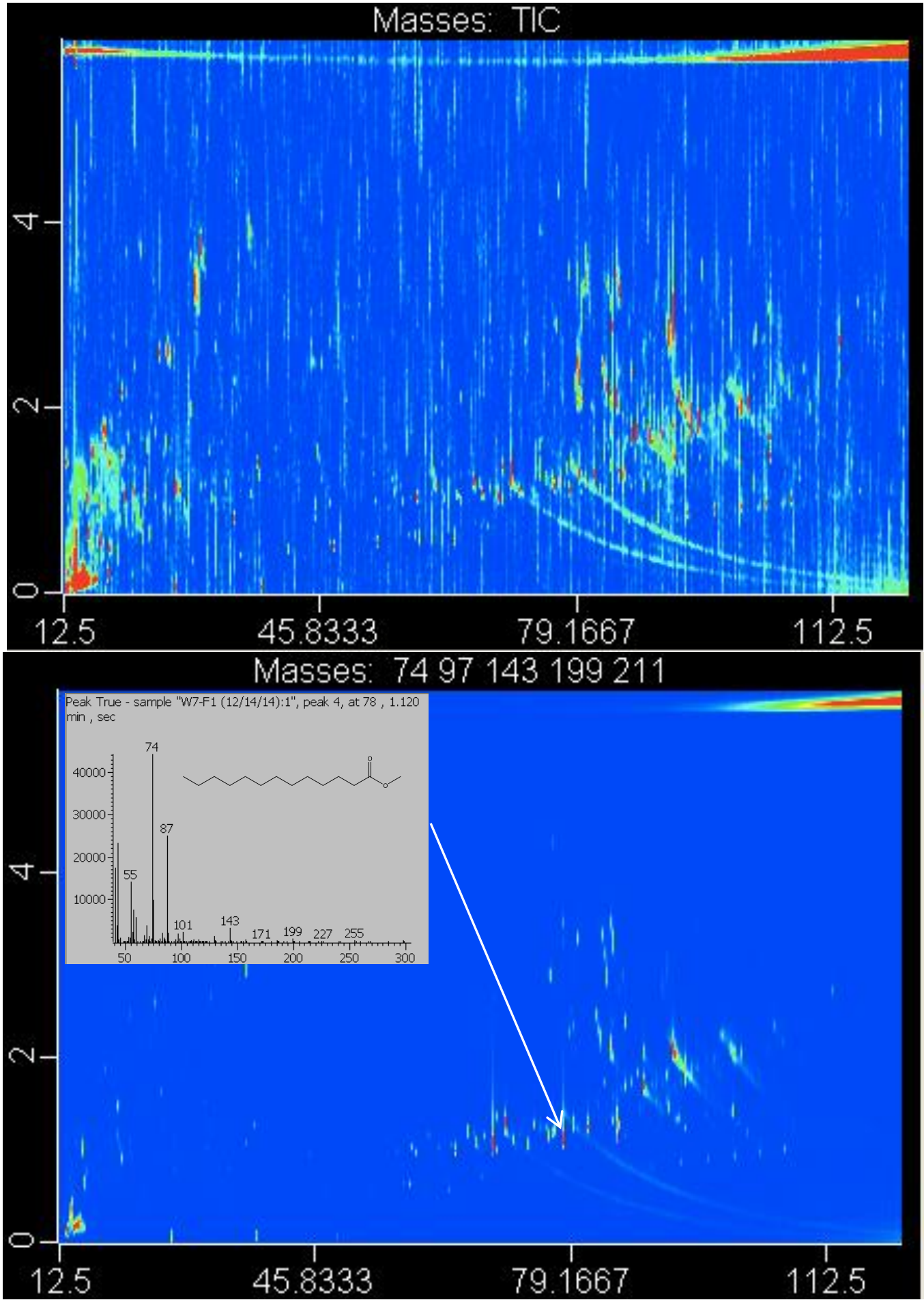

Figure S8. GC $\times$ GC-TOFMS total ion chromatogram (top) of tissue extract from worm incubated with C1800 sediment and reconstructed ion chromatogram (bottom) using $\mathrm{m} / \mathrm{z}$ values specific to fatty acid methyl esters (74, 97143199 and 211). Also shown is an 
example of a structure identified for one of the more abundant peaks - tridecanoic acid methyl ester. 
References

1. Smedes, F.; Geertsma, R. W.; van der Zande, T.; Booij, K., Polymer-water partition coefficients of hydrophobic compounds for passive sampling: application of cosolvent models for validation. Environ Sci Technol 2009, 43, (18), 7047-54.

2. Choi, Y.; Cho, Y. M.; Luthy, R. G., Polyethylene-water partitioning coefficients for parent- and alkylated-polycyclic aromatic hydrocarbons and polychlorinated biphenyls. Environ Sci Technol 2013, 47, (13), 6943-50.

3. Adams, R. G.; Lohmann, R.; Fernandez, L. A.; Macfarlane, J. K.; Gschwend, P. M., Polyethylene devices: Passive samplers for measuring dissolved hydrophobic organic compounds in aquatic environments. Environ Sci Technol 2007, 41, (4), 1317-1323.

4. $\quad$ Fernandez, L. A.; MacFarlane, J. K.; Tcaciuc, A. P.; Gschwend, P. M., Measurement of freely dissolved PAH concentrations in sediment beds using passive sampling with lowdensity polyethylene strips. Environ Sci Technol 2009, 43, (5), 1430-1436.

5. Hale, S. E.; Martin, T. J.; Goss, K.-U.; Arp, H. P. H.; Werner, D., Partitioning of organochlorine pesticides from water to polyethylene passive samplers. Environ Pollut 2010, 158, (7), 2511-2517.

6. Burns, S. T.; Khaledi, M. G., Rapid determination of liposome-water partition coefficients (K-lw) using liposome electrokinetic chromatography (LEKC). J Pharm Sci 2002, 91, (7), 1601-1612.

7. Acree Jr, W. E.; Abraham, M. H., Solubility predictions for crystalline polycyclic aromatic hydrocarbons (PAHs) dissolved in organic solvents based upon the Abraham general solvation model. Fluid Phase Equilibr 2002, 201, (2), 245-258.

8. Endo, S.; Escher, B. I.; Goss, K. U., Capacities of membrane lipids to accumulate neutral organic chemicals. Environ Sci Technol 2011, 45, (14), 5912-21.

9. van Noort, P. C. M.; Haftka, J. J. H.; Parsons, J. R., Updated Abraham solvation parameters for polychlorinated biphenyls. Environ Sci Technol 2010, 44, (18), 7037-7042.

10. Mintz, C. Predicting chemical and biochemical properties using the Abraham general solvation model. University of North Texas, 2009.

11. Endo, S.; Watanabe, N.; Ulrich, N.; Bronner, G.; Goss, K. U., UFZ Database v 2.1. In 2014 ed.; Helmholtz Center for Environmental Research - UFZ: Leipzig, Germany.

12. Abraham, M. H.; Poole, C. F.; Poole, S. K., Classification of stationary phases and other materials by gas chromatography. J Chromatogr A 1999, 842, (1-2), 79-114.

13. Nabi, D.; Gros, J.; Dimitriou-Christidis, P.; Arey, J. S., Mapping environmental partitioning properties of nonpolar complex mixtures by use of GC x GC. Environ Sci Technol 2014, 48, (12), 6814-26. 



\section{Chapter 4}

\section{Modeling the transport of organic chemicals between polyethylene passive samplers and water in finite and infinite bath conditions}

A. Patricia Tcaciuc ${ }^{1,2^{*}}$, Jennifer N. Apell ${ }^{2}$, Philip M. Gschwend ${ }^{1,2}$

${ }^{1}$ Department of Civil and Environmental Engineering, Massachusetts Institute of Technology, Cambridge, MA 02138, USA

${ }^{2}$ Department of Marine Chemistry and Geochemistry, Woods Hole Oceanographic Institution, Woods Hole, MA 02543, USA

Manuscript under review at Environmental Toxicology and Chemistry 


\begin{abstract}
Understanding the exchange kinetics of chemicals between passive samplers and water is essential for their use as monitoring devices of organic contaminants in surface waters. Here, we present a mathematical model for the uptake of chemicals into passive samplers from water, in either finite or infinite bath conditions. The solution was derived by applying Fick's $2^{\text {nd }}$ law to diffusion through the polymer membrane and through an aqueous boundary layer. The finite bath model performed well when applied to laboratory observations of sorption into polyethylene (PE) sheets for various chemicals (PAHs, PCBs and DDTs) and at varying turbulence levels. We also applied the model to field-deployed PE to infer fractional equilibration of PCB and DDT analytes, and the results were nearly identical to those obtained using the sampling rate model. However, further comparison of our model and the sampling rate model using mathematical simulations revealed that the polymer/water exchange kinetics were well described by an exponential only when the overall mass transfer was water-boundary-layer controlled. In contrast, the uptake or release of chemicals was not consistent with the sampling rate model when the transport was partially or fully membrane-controlled, which would be expected in turbulent conditions or when targeting compounds with small polymer diffusivities and small partition coefficients (e.g., phenols, some pesticides). Though derived and tested using PE, the model can be applied to other sampler materials, as well as other chemicals, and in any transfer regime (membrane, mixed or water-boundary-layer controlled). Lastly, we illustrate a few practical applications of this model such as improving passive sampler design and understanding the kinetics of passive dosing experiments.
\end{abstract}




\subsection{Introduction}

Passive sampling devices (PSDs) are increasingly being used for monitoring the freely dissolved concentrations of organic chemicals in surface waters. While various polymeric membrane samplers (e.g. polyethylene (PE) ${ }^{1-4}$, polyoxymethylene $e^{4-6}$, polydimethylsiloxane ${ }^{3,4,7}$, and others ${ }^{8,9}$ ) have been traditionally used for sampling hydrophobic organic chemicals (HOCs), researchers have recently applied them to emerging and moderately polar contaminants (e.g. nonylphenol and $\operatorname{triclos}^{10}{ }^{10}$, pyrethroids and organophosphates ${ }^{11}$ ). In addition to field applications, PSDs have recently been used in the laboratory for maintaining constant exposure levels of HOCs towards test organisms in toxicity assays (i.e. passive dosing). ${ }^{12,13}$ However, our current understanding of the PSD/water exchange kinetics is largely derived from studies targeting hydrophobic contaminants, such as PCBs and PAHs ${ }^{14-16}$ As we expand the use of PSDs to new chemicals and applications, we need a model that can be easily applied to different chemicals, sampler materials, and field/laboratory conditions.

Early efforts ${ }^{2}$ of modeling the exchange kinetics of polymer membranes in finite bath and infinite bath systems assumed that the transport was fully controlled by the membrane. Although mathematical descriptions for membrane-controlled transport in finite and infinite baths are readily available ${ }^{17}$, it was recognized that these formulations are often insufficient to describe the transport of chemicals between polymer membranes and water, especially in the case of hydrophobic chemicals ${ }^{18}$. In infinite and finite bath systems, the uptake rates were found to depend on hydrodynamic conditions, suggesting that the transfer was at least partially controlled by the water phase. ${ }^{3,19-22}$

In current passive sampling literature, the kinetics between PSDs and water are assumed to follow an exponential (Equation 4.1), which depends on a mass transfer coefficient $\left(\mathrm{k}_{\mathrm{o}}, \mathrm{cm} / \mathrm{s}\right)^{23}$ :

$$
C_{S}(t)=C_{W}^{0} K_{S W} f_{W}\left(1-e^{-\frac{k_{o} A t}{K_{S W} W_{S}} \frac{1}{f_{W}}}\right)
$$

where $C_{S}$ is chemical concentration in the sampler $\left(\mathrm{mol} / \mathrm{L}_{\mathrm{S}}\right), C_{W}$ is the initial concentration of the chemical in the water phase $\left(\mathrm{mol} / \mathrm{L}_{\mathrm{W}}\right), K_{S W}$ is the sampler-water partition coefficient $\left(\mathrm{L}_{\mathrm{W}} / \mathrm{L}_{\mathrm{S}}\right), A$ and $V_{S}$ are the sampler's surface area $\left(\mathrm{cm}^{2}\right)$ and volume $\left(\mathrm{cm}^{3}\right)$, respectively, and $f_{W}$ is the fraction of the chemical expected to be in the water phase at equilibrium. For infinite bath cases $f_{W} \rightarrow 1$ and Equation 4.1 reduces to the more familiar form found in several references ${ }^{14,15,20,21}$. 
Previous work ${ }^{15,18,24}$ assumed that the overall mass transfer coefficient $k_{o}$ is given by the inverse sum of the mass-transfer coefficients for the water $\left(k_{W}, \mathrm{~cm} / \mathrm{s}\right)$ and membrane $\left(k_{S}, \mathrm{~cm} / \mathrm{s}\right)$ phases:

$$
\frac{1}{k_{o}}=\frac{1}{k_{w}}+\frac{1}{k_{S} K_{S W}}
$$

Depending on the relative magnitudes of the water and membrane resistance, two regimes are identified. For aqueous control, the sampling rate $\left(R_{S}=k_{o} A\right)$, is weakly dependent on the compounds' hydrophobicity or molecular weight (for PE and SPMD $R_{S} \approx M W^{0.35}$ or $\approx K_{O W}{ }^{-0.044}$, Booij et al. ${ }^{15}$ ), whereas for membrane control, the sampling rate is more strongly dependent on hydrophobicity (for PE and SPMD $R_{S} \approx K_{O W}{ }^{0.69}$, Booij et al. ${ }^{15}$ ).

However, the mathematical description that allows the calculation of a sampling rate fundamentally relies on the assumption that the approach to equilibrium follows Equation 4.1. It is known, though, that for membrane controlled diffusion, the analytical solution is a sum of exponentials $^{2,25}$, because concentration gradients in the membrane lead to changes in $k_{S}$ over time. Furthermore, even in the long-time limit, when the membrane-controlled transfer also reduces to an exponential, the solution includes a pre-exponential term equal to $8 / \pi^{2}$ (Text $\mathrm{S} 1$, Equations 39 and 54, Crank ${ }^{17}$ ). In contrast, in Equation 4.1, the pre-exponential term is assumed to be 1, suggesting that Equation 4.1 may not accurately capture membrane-controlled transfer of chemicals.

Due to the nature of samplers used (thickness $<100 \mu \mathrm{m}$ ) and the chemicals (HOCs with log $\left.K_{O W}>4\right)$ targeted for passive sampling so far in the literature, Equation 4.1 was found satisfactory in most situations because the mass transfer was mainly water-side controlled ${ }^{24}$ and $k_{o}$ was equal to $k_{w}$. But some studies have identified situations where membrane-mediated transfer was applicable to at least a subset of the targeted chemicals ${ }^{15,20}$, and attempting to use only one model (i.e. either Equation 4.1 or the membrane-based solutions of Crank $^{17}$ ), did not properly capture the entire range of chemicals ${ }^{26,27}$. Furthermore, employing an inappropriate model for laboratory studies can lead to erroneous values of model-fitted polymer properties, such as diffusivities ${ }^{18}$. It has also been proposed $^{18}$ in a recent review that there may be future PSD applications where membrane control would be preferred (e.g. long term deployment of thick membranes in remote waters). 
Thus, the main goal of this work was to develop a more general mass transfer model for PSDs in water that takes into account diffusion through both the membrane and water, and compare it against existing models (Equations 4.1-4.2). The objectives of this study were to (i) test the analytical solution of the mass transfer model against laboratory sorption experiments performed with diverse HOCs and in various turbulence conditions, (ii) investigate when the assumption of exponential approach to equilibrium (i.e., the sampling rate method) is no longer adequate, (iii) apply the model to the loss of performance reference compounds (PRCs) from field deployed PE, and (iv) compare calculated fractional equilibrations against those derived based on sampling rate calibrations. Finally, we illustrate two model applications for optimization of passive dosing kinetics and field sampler design. Although we derive and test the model using PE, the solutions presented herein can be applied to other absorptive passive samplers.

\subsubsection{PE/water mass transfer - finite baths.}

By assuming the presence of an aqueous diffusive water boundary layer (WBL) on the outside of the polymeric sampler, and applying Fick's second law of diffusion to both the polymer and the WBL, we obtain the following governing equations:

$$
\begin{array}{ll}
\frac{\partial C_{P E}}{d t}=D_{P E} \frac{\partial^{2} C_{P E}}{\partial x^{2}} & \text { for }-L<x<L \\
\frac{\partial C_{W}}{d t}=D_{W} \frac{\partial^{2} C_{W}}{\partial x^{2}} & \text { for } L<x<L+\delta \text { and }-\delta-L<x<-L
\end{array}
$$

where $L$ is the PE half thickness, $\delta$ is the compound-specific WBL thickness, $D_{P E}$ and $D_{W}$ are the diffusivities in $\mathrm{PE}$ and water $\left(\mathrm{cm}^{2} / \mathrm{s}\right)$, respectively. We assume local equilibrium and no accumulation of mass of chemical at the PE-water interface $(x=L)$. In finite baths, the concentration in the water changes in response to the PE/water flux:

$$
\frac{\partial C_{W}}{d t}=-D_{W} \frac{A_{P E}}{V_{W}} \frac{\partial C_{W}}{\partial x} \text { at } x=-L-\delta \text { and } x=L+\delta
$$

where $A_{P E}$ is the area of PE, and $V_{W}$ is the volume of water.

The analytical solution is found by taking the Laplace transform of the equation and boundary conditions, which replaces the time variable with the Laplace parameter, $s$. When all the chemical is present in the water phase at $t=0$, the Laplace solutions (full derivation in Text $\mathrm{S} 1)$ for the concentration of chemical in PE and water are given by: 


$$
\begin{aligned}
& C_{P E}=C_{W}^{0} \frac{1}{s^{\frac{3}{2}}} \frac{K_{P E W}}{\operatorname{coth}(\sqrt{s})-\frac{K_{P E W}\left[1-\frac{L}{H} \sqrt{\frac{\psi}{s}} \operatorname{coth}\left(-\alpha \sqrt{\frac{s}{\psi}}\right)\right]}{\sqrt{\psi}\left[\operatorname{coth}\left(-\alpha \sqrt{\frac{s}{\psi}}\right)-\frac{L}{H} \sqrt{\frac{\psi}{s}}\right]}} \\
& \left.C_{W}=C_{W}^{0}\left(\frac{1}{s}-\frac{\frac{1}{s^{3 / 2}} K_{P E W} \frac{L}{H}}{\operatorname{coth}(\sqrt{s})-\frac{K_{P E W}\left[1-\frac{L}{H} \sqrt{\frac{\psi}{s}} \operatorname{coth}\left(-\alpha \sqrt{\frac{s}{\psi}}\right)\right]}{\sqrt{\psi}\left[\operatorname{coth}\left(-\alpha \sqrt{\frac{s}{\psi}}\right)-\frac{L}{H} \sqrt{\frac{\psi}{s}}\right]}}\right] \frac{1}{\left.\cosh \left(-\alpha \sqrt{\frac{s}{\psi}}\right)-\frac{L}{H} \sqrt{\frac{\psi}{S}} \sinh \left(-\alpha \sqrt{\frac{s}{\psi}}\right)\right]}\right)
\end{aligned}
$$

where $H$ is $V_{W} / A_{P E}, \psi$ is $D_{W} / D_{P E}$, and $\alpha$ is $\delta / L$. Equations 4.6 and 4.7 can readily be translated into time domain using numerical inversion algorithms in Matlab ${ }^{28}$ (Code S1), Mathematica, Python or other programing languages. Compared to other numerical models for PSDs in water 3, 19, this approach is less computationally demanding, as the inversion algorithm is very fast (evaluates Equations 4.6 and 4.7 for one time point in under $0.1 \mathrm{~s}$ ).

The boundary layer thickness $\delta$, is the only model parameter that cannot be measured directly, but it is generally dependent on the level of turbulence and the nature of the solute. To test the mass transfer model, we performed experiments in which we varied the turbulence and compound identity independently, and checked that the evolution of model-derived, and compound-specific $\delta$, was consistent with previously known theoretical or empirical relationships.

\subsubsection{PE/water mass transfer - infinite bath.}

For large volumes of water (i.e. infinite baths), the concentration in the well mixed water bath remains constant with time and the corresponding Laplace solutions for the concentration of PRC and target analytes in PE can be derived by taking the limit of Equation 4.6 as $\mathrm{V}_{\mathrm{W}} \rightarrow \infty$ (detailed derivation in Text S1):

$$
\begin{aligned}
& C_{\text {target }}^{P E}=C_{\text {target }}^{W} K_{P E W} \frac{1}{s^{\frac{3}{2}}} \frac{1}{\frac{\mathrm{K}_{P E W}}{\sqrt{\psi}} \tanh \left(-\alpha \sqrt{\frac{s}{\psi}}\right)-\operatorname{coth}(\sqrt{s})} \\
& C_{P R C}^{P E}=C_{P R C}^{P E, 0}\left(\frac{1}{s}-\frac{1}{s^{\frac{3}{2}}} \frac{1}{\frac{\mathrm{K}_{\mathrm{PEW}}}{\sqrt{\psi}} \tanh \left(-\alpha \sqrt{\frac{s}{\psi}}\right)-\operatorname{coth}(\sqrt{s})}\right)
\end{aligned}
$$

where $C_{P R C}^{P E, 0}$ is the initial concentration of compound in the PE, $C_{\text {target }}^{W}$ is the concentration of a target analyte in the well-mixed bath. Equation 4.8 successfully reduces, for $\delta=0$ to the Laplace 
solution for diffusion in a sheet from an infinite bath ${ }^{17}$, and both Equations 4.8 and 4.9 are in perfect agreement with recently published Laplace models ${ }^{29}$.

Although Equations 4.8 and 4.9 can be easily inverted to time domain (Matlab code provided in Code S2), the Laplace domain expressions offer limited physical understanding of the mass transfer. Thus, we attempted to simplify them by using a late time approximation for small values of $\mathrm{s}$, which is often used in the groundwater literature ${ }^{30}$. Using this approximation gives a simple time domain expression, which can be directly compared to the sampling rate model:

$$
\begin{aligned}
& C_{\text {target }} \approx C_{\text {target }}^{\infty}\left(1-e^{-\frac{t}{\frac{K_{P E W} L \delta}{D_{W}}+\frac{L^{2}}{3 D_{P E}}}}\right) \\
& C_{P R C} \approx C_{P R C}^{0} e^{\left(-\frac{t}{\frac{K_{P E W} L \delta}{D_{W}}+\frac{L^{2}}{3 D_{P E}}}\right)}
\end{aligned}
$$

Given that the correspondence between small $s$ and late time does not always hold ${ }^{30,31}$, we will refer to Equations 4.10 and 4.11 simply as exponential approximations (EA). Equation 4.10 describes an exponential similar to Equation 4.1, with the exchange coefficient in the case of EA equal to:

$$
k_{e, E A}=\frac{1}{L\left(\frac{K_{P E W} \delta}{D_{W}}+\frac{L}{3 D_{P E}}\right)}
$$

and in the case of Equation 4.1:

$$
k_{e}=\frac{1}{L\left(\frac{K_{S W}}{k_{W}}+\frac{1}{k_{S}}\right)}
$$

after substitution of Equation 4.2 and $V_{S} / A=L$, and plugging in $f_{w}$ of 1 . We will see that for water-side controlled transfer, the EA and the best fit exponential of Equation 4.1 are actually the same, but for mixed- and membrane-controlled transport, the assumption of exponential approach to equilibrium as described by Equation 4.1 is not appropriate.

\subsection{Materials and Methods.}

All solvents used in this study were purchased from VWR (JT Baker Ultraresi-analyzed). The water used in the sorption experiments was first treated with an ion exchange and activated 
carbon system (Aries Vaponics, Rockland, MA), then exposed to UV in a TOC reduction unit (Aquafine Corporation, Valencia, CA). Standards for phenanthrene, pyrene, PCBs (EPA PCB Congener Calibration Check Solution, RPC-EPA2-1) and organochlorine pesticides (a mixture of 2,4' and 4,4' isomers of DDT, DDE, and DDD, Ultra Scientific Organochloride Pesticide Mixture, RPM-838-1) were all purchased from Ultra Scientific. Isotopically labeled compounds were purchased either from Cambridge Isotope Laboratory (all ${ }^{13} \mathrm{C}$ labeled chemicals, Tewskbury, MA) or C/D/N Isotopes ( $\mathrm{d}_{8}-2,4$ ’ DDT, Pointe-Claire, Quebec).

Polyethylene strips were cut to desired size from PE sheets $(25 \mu \mathrm{m}$, Film Guard 1 mil plastic drop cloth, Covalence Plastics, Minneapolis, MN), and cleaned with dichloromethane (DCM), methanol and water, prior to being used in sorption experiments in the laboratory, or loaded with PRCs from an 80-20 mixture of methanol-water following the procedure of Booij et al. ${ }^{32}$

\subsubsection{Phenanthrene and pyrene uptake at variable stirring speeds.}

For the variation of the boundary layer thickness $\delta$ against turbulence we used phenanthrene and pyrene as test chemicals, both due to their relevance as environmental contaminants as well as the ability to easily measure their aqueous concentrations using fluorescence spectroscopy. Using fluorescence spectroscopy, the evolution with time of the aqueous concentrations of the two chemicals was measured as the two chemicals diffused from water into PE. Uptake profiles at various turbulence levels could then be fit with the finite bath model to determine the best fit boundary layer thicknesses for each level of turbulence.

A volume of $575 \mathrm{~mL}$ of Milli-Q water in a ground glass stoppered flask was spiked with phenanthrene and pyrene dissolved in methanol, to achieve an aqueous concentrations of 100 and $25 \mu \mathrm{g} / \mathrm{L}$, respectively. About $50 \mathrm{mg}$ of PE, skewered on a glass Pasteur pipette, was added to the flask, along with a glass-coated stirbar. The aqueous concentrations of phenanthrene and pyrene in the experimental flasks and in a no-PE control flask were monitored using synchronous fluorescence spectroscopy at intervals of $5 \mathrm{~min}$ to several h, until PE-water equilibrium was reached. During the experiments, $3 \mathrm{~mL}$ aliquots of the water were transferred to quartz cuvettes and analyzed using a Perkin Elmer luminescence spectrometer LS 50B, in synchronous fluorescence mode, scanning at $500 \mathrm{~nm} / \mathrm{min}$, using $55 \mathrm{~nm}$ offset, over a scan range from 250 to $350 \mathrm{~nm}$, and with $7 \mathrm{~nm}$ excitation and emission slit widths. The fluorescence intensities were measured for phenanthrene and pyrene at $292.4 \mathrm{~nm}$ and $318.93 \mathrm{~nm}$, respectively. Instrument 
error was calculated based on the standard deviation of three measurements at each time point $( \pm 3.6 \mu \mathrm{g} / \mathrm{L}$ for phenanthrene and $\pm 0.5 \mu \mathrm{g} / \mathrm{L}$ for pyrene). The aliquot of water $(\sim 3 \mathrm{~mL})$ used for fluorescence measurement was returned to the incubation flasks by pipetting it along the walls to avoid creating turbulence. The procedure was conducted at 60, 100 and $400 \mathrm{rpm}$ stirring speeds. No decrease in the concentration of the two chemicals in the control flasks was observed throughout the experiments.

PCBS and DDTs uptake by PE. To test the performance of the model when applied to multiple compounds with an environmentally relevant range of physico-chemical properties, uptake experiments from water into PE were conducted with PCBs and DDTs spanning a log $\mathrm{K}_{\mathrm{OW}}$ range from 4.5 to 7.4 and MW range of 223 to $395.5 \mathrm{~g} / \mathrm{mol}$. A volume of $400 \mu \mathrm{L}$ of 1000 $\mathrm{ng} / \mathrm{mL}$ solution of PCBs and DDTs in hexane was loaded on the walls of a pre-cleaned and dried $4 \mathrm{~L}$ glass amber bottle. The bottle was rolled for $5 \mathrm{~min}$ and allowed to sit for $15 \mathrm{~min}$ to ensure evaporation of the solvent. A volume of $4 \mathrm{~L}$ of Milli-Q water was then added and the bottle was capped and set on an orbital shaker for one week at $100 \mathrm{rpm}$. The solution was further diluted to $\sim 25 \mathrm{ng} / \mathrm{L}$ by splitting it into four other $4 \mathrm{~L}$ amber bottles. In each bottle, three $5 \mathrm{mg}$ pieces of PE, skewered on Pasteur pipettes at intervals of $\sim 3 \mathrm{~cm}$ apart, were added. The four bottles were then incubated on an orbital shaker at room temperature and one bottle was sacrificed at 1, 2, 4 and $8 \mathrm{~d}$. At the end of each incubation time, the three pieces of PE were removed and extracted three times with $15 \mathrm{~mL}$ of dichloromethane. The concentration in the water phase was also measured by extracting a volume of the water $(500 \mathrm{~mL}$ for $1 \mathrm{~d}, 750 \mathrm{~mL}$ of 2 and $4 \mathrm{~d}$ and $1.5 \mathrm{~L}$ for $8 \mathrm{~d}$ incubations). The liquid-liquid extraction was performed three times using a 10:1 water to DCM ratio and 5 min shaking time. Prior to the extraction step, the water aliquots from before and after the incubations, and the PEs were spiked with a known amount (2.5-10 ng each) of a mixture of surrogate standards $\left({ }^{13} \mathrm{C}\right.$ labeled PCB congeners 19, 52, 105, 167, 170, 194 and 2,4'DDT, 2,4'-DDE and 2,4'-DDD). The DCM extracts were then concentrated by rotary evaporation and the volume was reduced to $50-250 \mu \mathrm{L}$ using a gentle stream of nitrogen at room temperature. During the last step of volume reduction, the extracts were solvent exchanged to hexane. Prior to GC-MS analysis (see below), a known amount of injection compounds (2.5 $10 \mathrm{ng}$ each of PCB congeners $39,55,104,150,188$ ) was added to each extract. 


\subsubsection{Field deployment of PE.}

To test the performance of the model in field conditions, we applied the model to PRCloaded passive samplers deployed in a freshwater subalpine lake (Lake Maggiore, Italy), previously contaminated with DDT from the discharge of chemical waste by a chemical plant into one of the lake's tributaries. The deployment of samplers in the water column was part of a larger field exercise aimed to characterize the concentrations and distribution of DDT, as well as DDE and DDD in the lake, but this chapter will focus solely on the PRCs loss from the water column samplers. Specifically, we wanted to see how the model-derived boundary layers varied across the different PRCs in field conditions compared to laboratory conditions (see previous section), and the suitability of this model for inferring fractional equilibration of target analytes from measured PRC losses.

Prior to deployment in the freshwater subalpine lake, strips of $25 \mu \mathrm{m}$ PE were cleaned and preloaded with a series of PRCs $\left({ }^{13} \mathrm{C}\right.$ labeled PCB congeners 28, 52, 111 and 153, and 2,4'-DDT, 4,4'-DDE, 4,4'-DDD) using the method of Booij et al. ${ }^{32}$. A second thickness of PE was employed $(10 \mu \mathrm{m})$ but was loaded with a single PRC, ${ }^{13} \mathrm{C} 4,4$ ' DDE (courtesy of ENI Donegani Institute). The PE strips were mounted in small metal frames and deployed at $\sim 2 \mathrm{~m}$ depth and retrieved after 10 and 30 days. PE samples were spiked with 10 ng each of ${ }^{13} \mathrm{C}$ PCB $70,{ }^{13} \mathrm{C} \mathrm{PCB}$ 105, $\mathrm{d}_{8}$ 2,4'-DDT and $\mathrm{d}_{8}$ 4,4'-DDD surrogate standards and extracted according to the procedures described above. Injection compounds (10 ng each of ${ }^{13} \mathrm{C}$ PCB 97 and ${ }^{13} \mathrm{C}$ PCB 167) were added to PE extracts before GC MS analysis.

\subsubsection{GC-MS analysis.}

All PE and water extracts (from finite bath experiments with DDTs and PCBs, as well as field deployed PE), were analyzed using an Agilent 6890 GC equipped with a cold-on-column injector, and coupled with mass spectrometry (JEOL GCmate, JOEL Ltd, Tokyo, Japan), operated in selected ion monitoring (SIM) mode. The quantification and confirmation ions used were as follows: $235 / 237$ for 2,4'- and 4,4'- DDD and DDT, 318/320 for 2,4'- and 4,4'-DDE, 222/224 for dichlorobiphenyls, 256/258 for trichlorobiphenyls, 292/294 for tetrachlorobiphenyls, 326/328 for pentachlorobiphenyls, 360/362 for hexachlrobiphenyls, and 394/396 for heptachlorobiphenyls (PCB analyses performed and quantified by JNA). Usually, a volume 1 or $2 \mu \mathrm{L}$ of the sample extract in hexane was injected onto the column at $55^{\circ} \mathrm{C}$. The separation was 
done on a DB-5 XLB column (J\&W Scientific DB-XLB, $30 \mathrm{~m}$ x $0.32 \mathrm{~mm} \mathrm{ID,} 0.5 \mu \mathrm{m}$ film thickness), with a He column flow of $2 \mathrm{~mL} / \mathrm{min}$. For DDT analysis in the laboratory and field samples, the oven temperature was ramped from $55^{\circ} \mathrm{C}$ to $175^{\circ} \mathrm{C}$ at $15{ }^{\circ} \mathrm{C} / \mathrm{min}$, and then to 270 ${ }^{\circ} \mathrm{C}$ at $4^{\circ} \mathrm{C} / \mathrm{min}$, followed by a $12 \mathrm{~min}$ hold. For PCB analysis, the column flow was $1 \mathrm{~mL} / \mathrm{min}$ and the oven was programmed from $65{ }^{\circ} \mathrm{C}$ to $160{ }^{\circ} \mathrm{C}$ at $30{ }^{\circ} \mathrm{C} / \mathrm{min}$, from $160{ }^{\circ} \mathrm{C}$ to $255^{\circ} \mathrm{C}$ at $10^{\circ} \mathrm{C} / \mathrm{min}$, and lastly to $315^{\circ} \mathrm{C}$ at $10^{\circ} \mathrm{C} / \mathrm{min}$, followed by a $5.2 \mathrm{~min}$ hold.

\subsection{Results and Discussion}

\subsubsection{Model tests}

Uptake of phenanthrene and pyrene into PE. The solution to the finite bath model (Equation 4.6 inverted with Code S1) fit the individual sorption profiles of phenanthrene and pyrene well for all three stirring speeds tested (Figure 4.1, Figure S1, $R^{2}=0.98-0.99$ ). Our model also fit similar literature data better than previous interpretations proposed by Adams et al. ${ }^{2}$, who assumed that the transfer was fully membrane controlled (Figure S2, also ${ }^{27}$ ), and by Lohmann ${ }^{18}$ who used a two film model, but obtained a rather wide range of WBL thicknesses (30-140 $\mu \mathrm{m})$. Using our model (Equation 4.7 and Code S1) and a best fit WBL of 16-25 $\mu \mathrm{m}$, we were able to better capture the sorption profiles of Adams et al. ${ }^{2}$ (Figure S2), suggesting that transport can still be, at least partially, water-side controlled even in strongly stirred systems. For example, in the case of the three test compounds of Adams et al., ${ }^{2}$ evaluating the relative proportion of the approximate transfer resistances based on Equation 2 (and Equation 4.14, as discussed below) in the water $\left(\mathrm{r}_{\mathrm{W}}=\mathrm{K}_{\mathrm{PEW}} \delta / \mathrm{D}_{\mathrm{W}}\right)$ and in the membrane $\left(\mathrm{r}_{\mathrm{m}}=\mathrm{L} / \mathrm{D}_{\mathrm{PE}}\right)$, we find that both phases contribute to the transfer (i.e. $r_{w}$ and $r_{m}$ do not differ by more than an order of magnitude, but instead, $\mathrm{r}_{\mathrm{W}}: \mathrm{r}_{\mathrm{m}} \sim 3: 1$ for $\mathrm{PCB} 52, \sim 1: 1$ for pyrene and $\sim 1: 2$ for phenanthrene). But, more importantly, we note that, for all three chemicals, assuming fully membrane controlled transfer will lead to estimated polymer diffusivities that are 1-2 orders of magnitude too low (Figure S2).

Effect of stirring speed. The rates of approach to polymer-water equilibrium increased with stirring speed for both test compounds, phenanthrene and pyrene, which is consistent with waterside controlled uptake (Figure 2). The value of the best fit WBL thickness (Figure 4.2) for phenanthrene and pyrene decreased with stirring speed, from 255 and $275 \mu \mathrm{m}$, respectively, in the $60 \mathrm{rpm}$ flask (gently stirred) to 80 and $90 \mu \mathrm{m}$, respectively, in the $400 \mathrm{rpm}$ flask (vigorous stirring, but without visible PE movement). These WBL thicknesses are consistent with values 


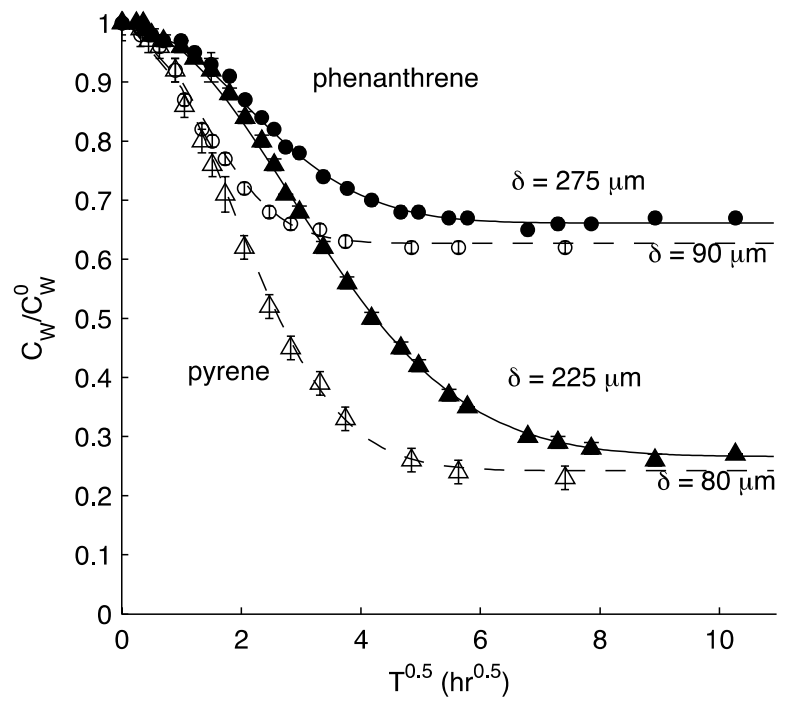

Figure 4.1 Results for phenanthrene (circles) and pyrene (triangles) uptake by PE from finite bath absorption experiments performed at two different stirring speeds $60 \mathrm{rpm}$ (filled symbols) and $400 \mathrm{rpm}$ (empty symbols). Lines represent finite bath model fits (Equation 4.7, inverted with Code S1) for $400 \mathrm{rpm}$ (dash lines) and $60 \mathrm{rpm}$ data (solid lines) with best fit WBL thickness displayed next to each profile. Data beyond $120 \mathrm{~h}$ are not shown because PE/water equilibrium was reached. Error bars represent error propagation of one SD of triplicate fluorescence intensity readings. For clarity, data from $100 \mathrm{rpm}$ experiment are not shown but can be found in Figure S1.

previously found in stirred systems ${ }^{18,19,22}$. Using a least square fit of the WBL thicknesses for phenanthrene and pyrene, we found WBL to be proportional to the stirring frequency $(N)$ to the power of $-0.60 \pm 0.04$ (Figure 4.2). For a laminar boundary layer, given that $\delta$ is proportional to the Reynolds number, $R e^{-0.5}$ for laminar flow over a flat plate, and $R e$ is proportional to $N$ we expect the best fit $\delta$ to be proportional to $N^{0.5}$. Thus, the exponent of -0.6 is consistent with the presence of a laminar boundary layer at the PE surface, as opposed to a turbulent one (for which $\left.\delta \approx N^{-1}\right)^{33}$. However, we recognize that the exponent of -0.6 could also reflect a transition between the two transfer regimes (laminar and turbulent boundary layers) that occurs across the range of stirring speeds tested and results in a best-fit exponent that is intermediate between the theoretical exponents of the stirring speed, $N$ of -0.5 (laminar boundary layer) and -1 (turbulent boundary layer). 


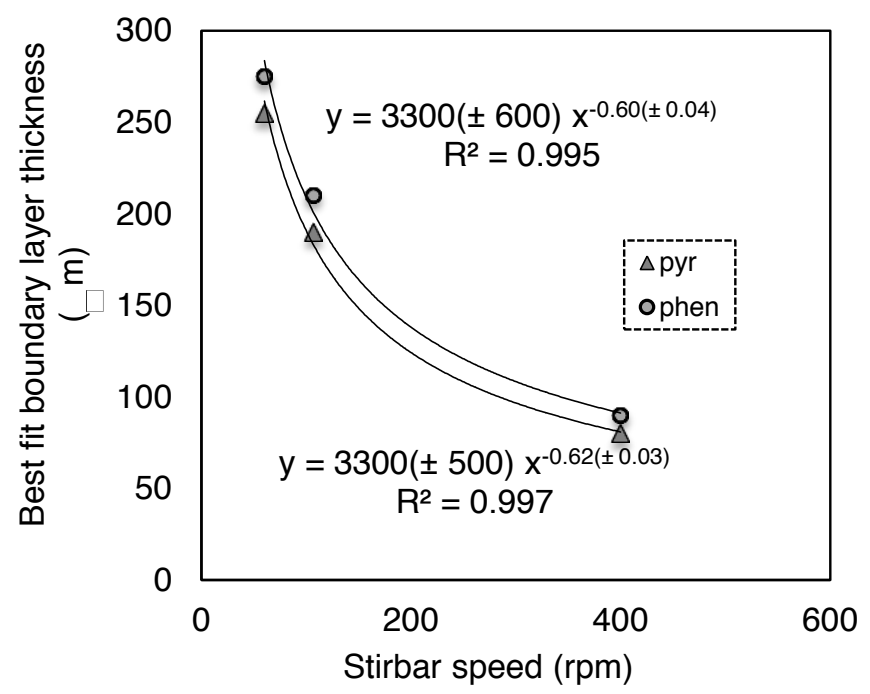

Figure 4.2 Best fit boundary layer thicknesses evaluated from PE absorption experiments for phenanthrene (circles) and pyrene (triangles) as a function of the stirring speed. Theoretical considerations (see text) dictate that the exponent should be -0.5 .

Uptake of PCBs and DDTs into PE. The finite bath model (Equation 4.6, Code S1) captured the PE uptake of various DDTs and PCBs (Figure 4.3), with best fit WBL thickness ranging from 17.5 to $23 \mu \mathrm{m}$. The only exceptions were the later time points for large PCBs, for which the amount accumulated in the PE in the near-equilibrium portion of the uptake curve was smaller than expected based on each compounds' $K_{P E W}$. This could be due to (a) the association of these compounds with colloids or dissolved organic carbon that may have been present in the water, or (b) uncertainty in the $K_{P E W}$ 's used in the model. It should be noted that using experiment derived $K_{P E W}$ values (Table $\left.\mathrm{S} 1\right)^{34,35}$, as opposed to ones derived from $K_{O W}$ - based regressions, led to better fits of the uptake curves, but experimental values were not available for all the PCBs used in the experiment. Lastly, the best fit boundary layers (17.5 to $23 \mu \mathrm{m})$ were lower in the $\mathrm{PCB} / \mathrm{DDT}$ experiments than in the phenanthrene/pyrene experiments, which is consistent with a higher level of turbulence in the PCB/DDT experiments. Compared to the most vigorous agitation in the phenanthrene/pyrene experiments at $400 \mathrm{rpm}$ via a $2 \mathrm{~cm}$ stir bar, which did not disturb the surface of the water, the agitation at $100 \mathrm{rpm}$ on the shaker table happened on a 5.1 $\mathrm{cm}$ orbit and imparted impart visible movement to the entire water volume.

No significant membrane contribution was observed in either the PCB/DDT or the phenanthrene/pyrene uptake experiments. The goodness of fit in Figures 4.1 and 4.3 was independent of the value of polymer diffusivity (e.g., lowering the polymer diffusivities of 
phenanthrene and pyrene by 50 fold compared to the measured or calculated values from Lohmann, ${ }^{18}$ only changed the model calculated fractional equilibration in Figure 4.1 by 0.02 at most, less than the measurement uncertainty), but instead depended strongly on the value of WBL thickness (e.g., changing $\delta$ by a factor of 5 changed the fractional equilibration by as much as 0.20 ). To test that our finite bath model could be used in membrane and mixed-control situations, we applied it to PAH uptake into POM data found in the literature ${ }^{26}$. Our model was able to capture the uptake into POM of naphthalene $\left(R^{2}=0.981\right)$ which was fully membrane controlled, of benz $[a]$ anthracene $\left(R^{2}=0.992\right)$ which was predominantly WBL controlled, as well the uptake of phenanthrenene $\left(R^{2}=0.972\right)$, which was mixed-membrane-WBL controlled (Figure S3). This is an improvement over the sampling rate model $^{23}$, which was not able to fit the uptake profiles of all three chemicals.

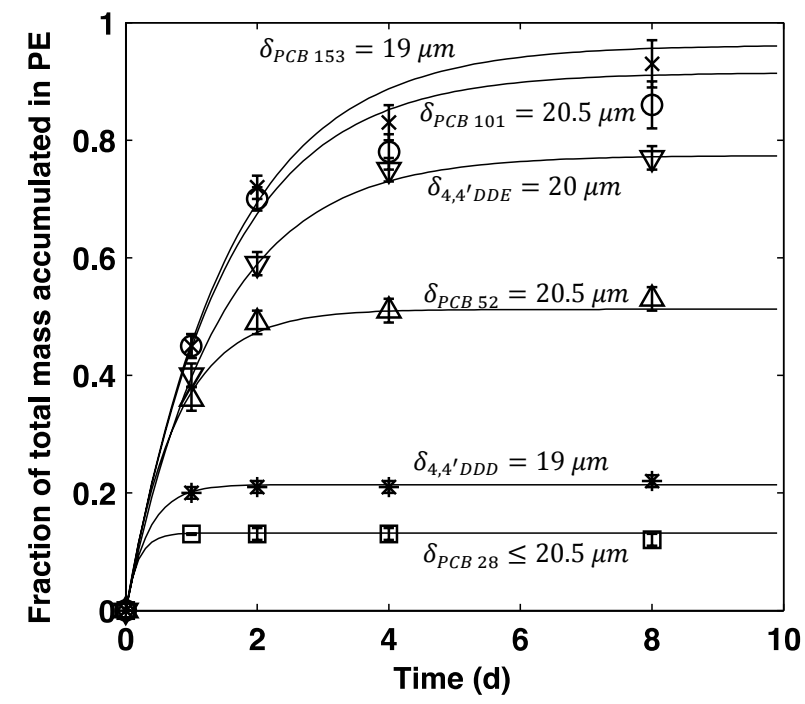

Figure 4.3 Model fit (Equation 4.6 inverted with Code S1) results for finite bath sorption experiments illustrated for select compounds: PCB 153 (crosses), PCB 101 (circles), 4,4'-DDE (down triangles), PCB 52 (up triangles), 4,4'-DDD (stars), PCB 28 (squares). The best fit boundary layer thickness is displayed next to each sorption profile. Error bars represent error propagation of one SD of measurement uncertaintity.

Effect of solute size. Lastly, we evaluated the robustness of the model by evaluating the dependency of the best fit boundary layer on molecular size. From theoretical considerations, as well as empirical fits of benthic boundary layer thicknesses near sediment surfaces ${ }^{36}$, we know 
that the mass transfer coefficient through the diffusive sublayer is proportional to Schmidt number to the $-2 / 3$. Assuming $D_{W}$ is proportional to $M W^{0.71}$ or $\bar{V}^{-0.589} 37$, and that the transfer velocity is given by $D_{W} / \delta$, this means that WBL thickness should be weakly inversely proportional to molecular size $\left(\delta \approx M W^{0.24}\right.$ or $\left.\approx \bar{V}^{-0.20}\right)$.

The best fit boundary layers deduced from the finite bath uptake experiments showed a weak dependency on molecular weight (Figure S4). For 17 PCBs and 6 DDTs, the best-fit $\delta$ was proportional to $M W^{0.42}\left(p<0.05,95 \% \mathrm{CI}-M W^{0.18}\right.$ to $M W^{0.66}$, Figure S4), which is consistent with the empirically derived value of -0.24 from other studies ${ }^{36}$. The fitted WBL values are subject to imperfect knowledge of compounds' $D_{W}$ and $K_{P E W}$ values. For example, in Figure S4, all the 2,4' isomers of DDT, DDD and DDE, appear to have larger best fit WBL values than the 4,4 ' isomers, possibly due to them having different aqueous diffusivities that may not be accurately captured by either a $M W$ or Hayduk-Laudie LeBas volume-based calculation ${ }^{37}$ (molar volumes calculated using SPARC) ${ }^{38}$. If we eliminate the deviating points from Figure $\mathrm{S} 4$, the $R^{2}$ goes up to 0.80 , without a significant change in the fit coefficients $\left(\delta \approx M W^{0.47 \pm 0.06}\right)$.

\subsubsection{Comparison with sampling rate model}

Mass transfer simulations using the infinite bath model (Equation 4.8 and Code S2) of various chemicals between PE and water were used to determine if the transport of chemicals was always governed by an exponential of the form of Equation 4.1. We used a data set of 107 nonpolar chemicals (19 PAHs, 20 organochlorine pesticides, 45 PCBs, 14 PBDEs and other organic chemicals), whose physico-chemical properties such as $D_{P E}$ and $K_{P E W}$ were recently reviewed ${ }^{18}$. The compound set also allowed us to identify situations when PE or WBL were controlling the overall diffusive exchange. In general, the transfer is water boundary layer controlled if:

$$
\frac{\delta}{D_{W}} \gg \frac{L}{D_{P E} K_{P E W}}
$$

For each compound in the critical review ${ }^{18}$, we produced an uptake profile as a function of time (Equation 4.8 inverted with Code S2) for a $100 \mu \mathrm{m}$ PE and a $20 \mu \mathrm{m}$ WBL. Diffusivity in water for each chemical was calculated based on its molecular weight ${ }^{37}$, with the caveat noted 
earlier that this method may not capture isomer specific effects. To this profile, we fit Equation 4.1 and separately evaluated the exponential approximation (Equation 4.10) at each time point.

For WBL-controlled chemicals, we found that all three profiles (full solution, exponential approximation and the best fit exponential of Equation 4.1) agreed perfectly (Figure 4.4A). The critical $K_{P E W}$ value required for WBL-controlled transport depended on the ratio of $\delta / L$ in accordance with Equation 4.14 (e.g., for a $100 \mu \mathrm{m}$ thick PE, we expect WBL control for $K_{P E W}>$ $10^{5}$ given $\delta=10 \mu \mathrm{m}$ and for $K_{P E W}>10^{3}$ given $\delta=500 \mu \mathrm{m}$, Figure S5). Therefore, for waterside controlled transport $\left(K_{P E W}>10^{5}\right)$, the exponential approximation is practically identical to the full solution (implying $k_{w}=D_{w} / \delta$ ), and the sampling rate approach is consistent with the model presented in this paper.

When the sampler membrane partially or fully controls the mass transfer, the sampling rate model does not perform well (e.g., deviations in fractional equilibrations calculated using each model of up to 0.1 , or a factor of 2, Figure 4.4B and 4.4C). For full membrane control, this is not surprising given that Equation 4.1 is missing a pre-exponential term compared to the membrane solution given by $\mathrm{Crank}^{17}$ (also in Text S1). Chemicals are more likely to be membrane controlled as $D_{P E}$ decreases (Figure 4.4 compare A and B), $K_{P E W}$ decreases (Figure $4.4 \mathrm{C}$ ), or the ratio of WBL to the half PE thickness $(\delta / L)$ decreases. It is worth noting that the best fit exponential (as would be obtained by employing Equation 4.1) and the exponential approximation (Equation 4.11) yield nearly identical uptake profiles (Figure 4.4 and Figure S6, $\mathrm{k}_{\mathrm{EA}} / \mathrm{k}_{\mathrm{fit}} \sim 0.999 \pm 0.002$ ), but these two uptake profiles are different from the full solution (Equation 4.8), particularly for fractional equilibrations $<0.5$. The deviations in fractional equilibrations between the full solution and the EA approximation were larger ( $\Delta$ of up to 0.1 , or a factor of 2) in the early part of the uptake profiles (Figure 4.4C) and increased with decreasing $K_{P E W}$ (Figure S5). While such differences between the two models may seem negligible, they may be relevant in the interpretation of field data. For example, if one was to use a PRC whose release is at least in part membrane controlled, but interpret the release as a simple WBLcontrolled exponential, the equilibrium concentration for the more hydrophobic compounds would be under- or over-estimated by factors of 2-30 (Figure S7). 

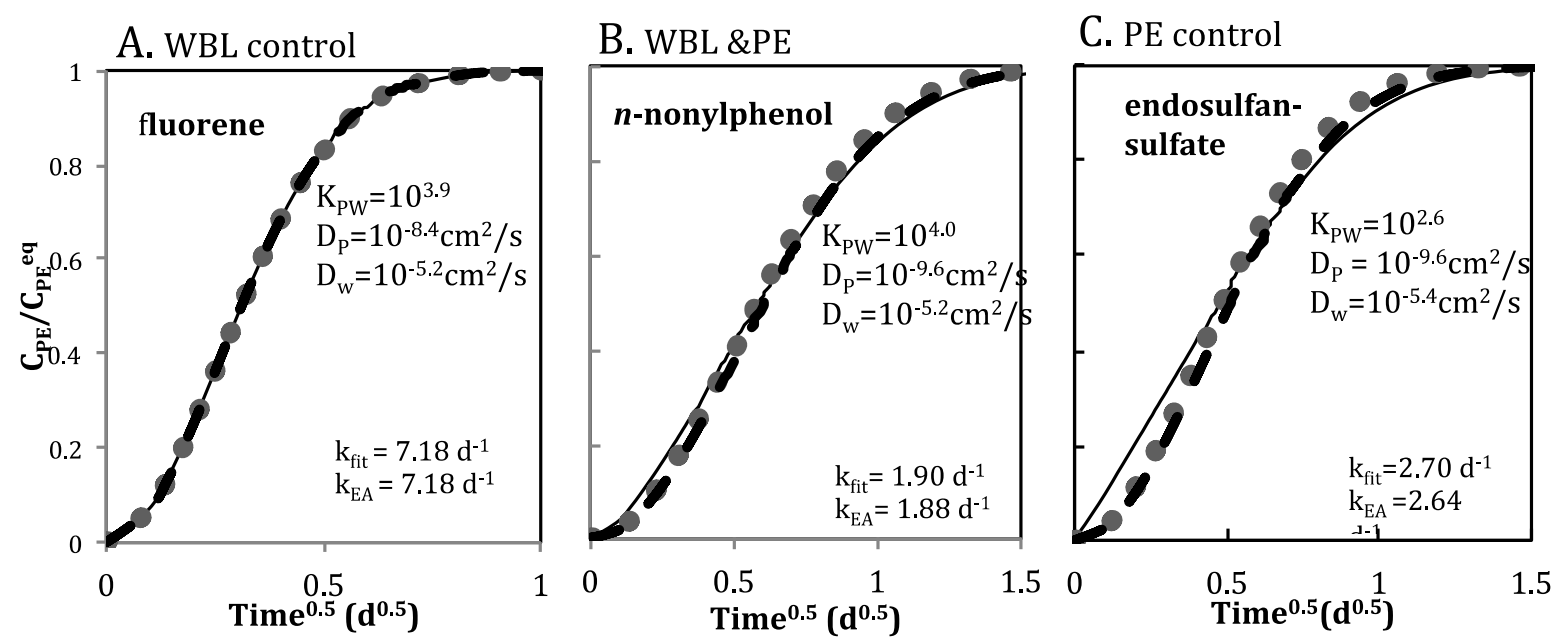

Figure 4.4 Illustration of three cases of mass transfer between PE and and an infinite water bath using three model compounds from Lohmann ${ }^{18}$, a $100 \mu \mathrm{m}$ thick PE and $20 \mu \mathrm{m}$ WBL. In each panel, the solid lines are the full solution of the model presented in this paper (Equation 4.8 inverted numerically with Code S2), the dotted line is the best fit exponential (kfit), assuming Equation 4.1 governs the mass transfer, and the dashed line is Equation 4.10 (plotted using kEA). The dashed line and dotted lines overlap in all three cases, suggesting that the exponential approximation is essentially the same as a best fit exponential. However, Equation 4.1 coincides with the full solution only when the transfer is WBL controlled (panel A, the only case when all three lines overlap).

Although it is generally thought that diffusion through the sampler membrane is only important in turbulent aqueous environments and for compounds with $K_{P E W}<10^{5}$, we find that $K_{P E W}$ is not the only parameter that determines if the transport is membrane or WBL controlled. As shown in Figure $4.4 \mathrm{~A}$ and B, fluorene and $n$-nonylphenol have very similar $K_{P E W}$ values, but due to their different $D_{P E}$ 's, the transport is WBL controlled for fluorene and partially membrane controlled for $n$-nonylphenol. This is understandable because in a series of chemicals such as PCBs and PAHs, a decrease in $K_{P E W}$ is accompanied by a decrease in the compound's molar volume and hence, an increase in its diffusivity in PE. In contrast, chemicals with oxygen atoms, such as phenols and some pesticides, have low $K_{P E W}$ 's, but they also have low $D_{P E}$ 's due to their large molar volume. Thus, membrane controlled transport may apply to many contaminants of emerging concern which are sufficiently hydrophobic to accumulate in nonpolar samplers ${ }^{39}$ but also have chemical structures containing oxygen and nitrogen atoms (compounds in select pesticides, biocides, personal care products or munitions).

Although we do not show a full comparison of our finite bath model against the sampling rate expression (Equation 4.1), similar considerations as in the case of the infinite bath model 
apply. The sampling rate expression is consistent with our model (Equation 4.6 - 4.7) only for WBL controlled situations. In membrane controlled situations, the sampling rate formulation does not agree with previously derived solutions for diffusion in a sheet from stirred solutions ${ }^{17}$ (Text S1, Equation 39), which was also apparent in the application of our model to Hong et al. ${ }^{26}$ data (Figure S3).

Lastly, we note that, similarly to the sampling rate, $\mathrm{R}_{\mathrm{S}}$, the WBL thickness is ultimately a modeling construct. As such, alternate models can be formulated, which do not assume the presence of a stagnant boundary layer on the outside of PE, but rather the continuous renewal of the water in contact with PE with fluid from the well mixed bath (similar to the air-water exchange surface renewal model). We thus consider briefly the implications for the dependency of the mass transfer on solute size if the PE/water transfer were characterized by a surface renewal model. We first assume that a surface renewal model would imply that the transfer velocity would have a dependency on the solute's diffusivity of $\mathrm{D}_{\mathrm{W}}{ }^{1 / 2}$. In order to compare this situation to the boundary layer model described so far, we will also assume that $\mathrm{k}_{\mathrm{W}, \mathrm{SR}}=\mathrm{D}_{\mathrm{W}} / \delta_{\mathrm{SR}}$, which implies that the hypothetical boundary layer thickness characteristic of a surface renewal model, $\delta_{\mathrm{SR}}$, would be proportional to $\mathrm{D}_{\mathrm{W}}^{1 / 2}$. Then, using $\mathrm{DW} \approx \mathrm{MW}^{-0.7}$ implies that $\delta_{\mathrm{SR}} \approx \mathrm{MW}^{-0.35}$. This result is not very different than that obtained for the boundary layer model of $\delta \approx \mathrm{MW}^{-0.24}$, and in fact, we notice that the relationship for $\delta_{\mathrm{SR}}$ is closer to the observed dependency of $\delta \approx \mathrm{MW}^{-0.42}$ from the PCB/DDT experiments. However, as we will see in the following section, in field conditions, the difference between the two models will likely be smaller than the uncertainties associated with environmental sampling.

\subsubsection{Model Applications}

Application of infinite bath model to field data. Using the PRC data from the field deployment of $10 \mu \mathrm{m}$ - and $25 \mu \mathrm{m}$-thick PE, we applied the model to infer fractional equilibrations of various target compounds and compared them against those derived based on sampling rate calibrations. The WBL thicknesses calculated based on PRC loss from fielddeployed PE (Equation 4.9 and Code S2), showed a very weak dependency on $M W$ (Figure S8). When using only PRCs which were lost more than 5\% ( $N=6$, three PCBs and three DDTs), we found no statistically significant dependency of $\delta$ on $M W$ for either the 10 day or the 30 day deployment (i.e., fitting $\delta=m^{*} M W^{n}$ fit, the $p$ values for the $n$ coefficient were $p=0.075$ and $p=$ 
0.43, respectively). Since the percentage loss of PRCs from both thicknesses of PE samplers could be explained to within 5\% using the average $\delta$ value of $170 \mu \mathrm{m}$ (Figure 4.5), we used this average WBL value and Equation 4.8 to evaluate the predicted fractional equilibration of various target chemicals. The only PRC whose loss deviated from the model was PCB 111. The model is sensitive to $K_{P E W}$ values (e.g., for every increase of $0.1 \log$ units in $K_{P E W}$, the percentage of PRC remaining predicted by the model can change by 5 to $10 \%$ ), and we attributed the deviation between model prediction and observations to uncertainty in the $K_{P E W}$ of PCB 111, for which no experimental value was found in the literature and $K_{O W}$-based predictions vary by $0.3 \log$ units.

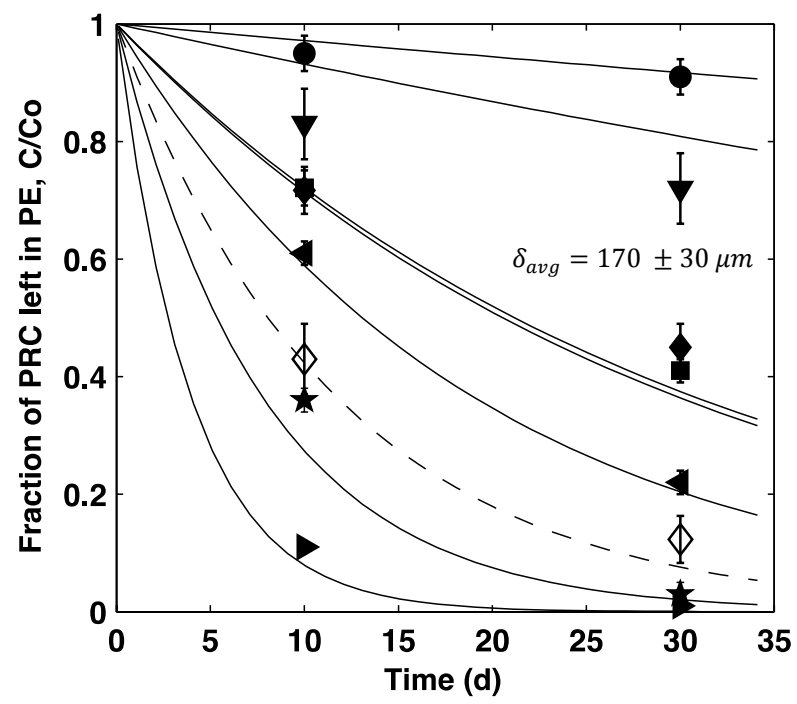

Figure 4.5 Measured fractions of PRCs left in field deployed PE of two thicknesses $25 \mu \mathrm{m}$ (filled symbols) and $10 \mu \mathrm{m}$ (empty symbols), after 10 day and 30 day deployments for ${ }^{13} \mathrm{C}$ PCB 153 (circles), ${ }^{13} \mathrm{C}$ PCB 111 (down triangles), ${ }^{13} \mathrm{C} 4,4$ '-DDE (diamonds), ${ }^{13} \mathrm{C} 2,4$ '-DDT (squares), ${ }^{13} \mathrm{C}$ PCB 47 (left triangles), ${ }^{13} \mathrm{C}$ PCB 28 (pentagram) and ${ }^{13} \mathrm{C} 4,4$ '-DDD (right triangles). The lines represent the corresponding model predictions of fractional PRC remaining in $25 \mu \mathrm{m}$ (solid lines) and $10 \mu \mathrm{m}$ (dashed line) thick PE, assuming an average water boundary layer thickeness $\delta_{\text {avg }}$ of $170 \pm 30 \mu \mathrm{m}$ (average and standard deviation of WBLs calculated from each PRC loss at each timepoint). Error bars calculated based on one SD of instrumental error and one SD of the pre-deployment PRC concentration.

As individual PRC-derived sampling rates showed no statistically significant dependency on $M W$ or $K_{P E W}$ (Figure $\mathrm{S} 9$, slopes of $\log \mathrm{R}_{\mathrm{S}}$ versus $\log \mathrm{MW}$ and $\log \mathrm{K}_{\mathrm{PEW}}$ have $p>0.05$ ), we used the nonlinear least squares (NLS) regression method of Booij and Smedes ${ }^{40}$ (Figure S10), to obtain a best fit $R_{S}$ of 3.5 (95\% C.I. 3.3-3.8) L/d for the 10 day and 3.4 (95\% CI 3.1-3.7) L/d for the 30 day deployment. Applying this to the same target compounds, we find that the sampling 
rate approach with NLS and the WBL model on average to within 1\% (Figure S11), which is in accordance with the fact that for WBL-controlled transport, the model presented in this paper and the sampling rate approach are identical.

Using finite bath model to optimize PSD/water transfer kinetics. Polymer membranes have also been employed in toxicity tests for buffering the loss of hydrophobic chemicals due to sorption, organism uptake, or degradation ${ }^{12,13}$. Such an application depends in part on the ability of the sampler to buffer chemical activity changes. Based on results from the finite bath model, the key parameters that affect the response time of the polymer are the PE/water ratio, the membrane and boundary layer thicknesses, and to some extent $K_{P E W}$ (Figure S12). For large $\mathrm{PE} /$ water ratios (fraction of chemical in the water at equilibrium, $\mathrm{f}_{\mathrm{i}, \mathrm{w}}<0.05$ ), the response time (time to reach $95 \%$ of equilibrium) for chemicals with $K_{P E W}$ ranging from $10^{4.1}$ to $10^{6.9}$, was hours or less. The fastest response time $(0.1-0.2 \mathrm{hr})$ was achieved with thin PE $(10 \mu \mathrm{m})$ and minimal $\delta(25 \mu \mathrm{m})$. Increasing either the PE thickness or $\delta$ by a factor of 10 , increases the response time to 1-2 $\mathrm{h}$, and increasing both leads to response times of 10-20 h. In contrast, a two order of magnitude variation in the polymer diffusivity for each chemical, did not affect the response time (Figure S12-D), suggesting minimal gains from using polymers with larger diffusivities. The model outputs are consistent with observations of equilibration times of min to hours for PAHs desorbing from PDMS films ${ }^{12,13}$.

Implications for field deployments. Deployment times for passive samplers measurements of HOCs in surface waters can be on the 2-8 weeks ${ }^{3,41,42}$ Such long deployment times, however, increase the likelihood of biofouling and sampler loss. To address this, we use our model to look at two scenarios that can decrease the deployment time. First, we ask the question is it possible to design a sampler that achieves close to $90 \%$ equilibration in a few days? Applying the infinite bath model to equilibrium sampling, we find that in order to sample compounds with $K_{P E W}$ less than $10^{7.5}$, such that they achieve equilibrium in a few days, one would need a $0.1 \mu \mathrm{m}$ thick sampler, assuming $\delta$ of $100 \mu \mathrm{m}$. Even in a turbulent environment ( $\delta=25 \mu \mathrm{m})$, the sampler would have to be $0.5 \mu \mathrm{m}$ thick, which (1) is thinner than any PSD currently in use and (2) would require a considerably wider sampler to achieve the same detection limits as a more commonly used sampler of $50 \mu \mathrm{m}$. An alternative approach would be to envision a device that keeps the sampler in motion or induces water flow, therefore decreasing boundary layer thickness to 
minimal values (order of 10-20 $\mu \mathrm{m}$ ). Even so, only compounds with $K_{P E W}$ less than $10^{6.5}$ would be fully equilibrated after a few days (assuming $10 \mu \mathrm{m}$ thick PE).

An alternative scenario to decrease deployment times would be to employ fast exchanging PRCs to infer the fractional equilibration of HOCs in kinetic sampling. To do this, one could use thick and/or large-area membranes, able to accumulate a detectable amount of HOCs, and loaded with small PRCs, whose loss can be used to calibrate the sampler (i.e. infer WBL thicknesses). The thickness of the PE would be tuned primarily for achieving a PRC loss that can be measured confidently but the thickness over width ratio should be smaller than 0.2 to avoid edge effects (i.e., the violation of the model assumptions of one dimensional diffusion in the polymer and one dimensional flux across PE/water interface). ${ }^{17}$ Our model is well suited for such an application because for small chemicals and thick membranes, the transport may be partially membrane controlled, and accurate $\delta$ determinations in that case are critical for the extrapolation from small PRC compounds to highly hydrophobic target analytes.

While both the sampling rate model and the model presented here can be used to infer the fractional equilibrium of target analytes based on PRC loss, our model has the advantage that it is applicable in all transfer regimes. In contrast, in the sampling rate model, $R_{S}$ versus $K_{O W}$ or $M W$ relationships are used to evaluate the sampling rate of target analytes, and these relationships are different for membrane or WBL-controlled uptake. Transfer regime may be difficult to identify as it depends on the chemical, the polymer used and the hydrodynamic conditions. For compounds with $K_{O W}$ values larger than $10^{4.5}$, the transfer is assumed to be in aqueous control regime ${ }^{15}$, but the value of the transition point depends on the polymer used (e.g. $\mathrm{K}_{\mathrm{OW}}$ of $10^{3}$ for silicone rubber ${ }^{16}$ versus $10^{4.5}$ for $\mathrm{PE}^{15}$ ) and on hydrodynamic conditions. ${ }^{40}$ It should also be noted that membrane-controlled chemicals diffusing into PE reach equilibrium on timescales of hours or days (Figure 4.4B and C) and thus, may not require an uptake model for typical deployment times (e.g. months). However, when using polymers with lower diffusivities such as POM, the equilibration times will be on the order of months and an uptake model will be required to infer fractional equilibration of target analytes.

Model significance. The comparison between our model and the sampling rate model for both finite and infinite bath situations showed significant overlap for WBL controlled chemicals, implying that either model (Equations 4.1-4.2 or Equations 4.6-4.9) can be used with the same 
expected accuracy. For membrane controlled chemicals, the sampling rate exponential (Equation 4.1 ) is not applicable, and the membrane solutions given in Crank ${ }^{17}$ should be used instead (also see Figure S13 for a more detailed analysis). While some generalization could be made regarding which compounds are always WBL controlled, we propose that Equation 4.14 is a better decision tool than the previously identified $K_{O W}$-based criteria, because Equation 4.14 takes into account compound and polymer specific factors, as well as environmental factors.

Compared to existing models, which can be used accurately in particular transfer regimes, the advantage of the model presented in this paper is its generality (can be used in WBL, mixed and membrane control regimes, and in finite or infinite bath situations). Even though it involves a numerical inversion step, the algorithm for the inverse Laplace transform is very fast compared to finite difference models. Furthermore, the model presented in this paper lays a simple theoretical foundation for passive sampling whose complexity can be gradually increased. For example, with appropriate modifications, similar mathematical approaches can be used to model how the sampling kinetics may be affected by the presence of organic carbon (dissolved or particulate) or NAPLs in the water, or by sampler biofouling during deployment. Lastly, the model has potential for application outside the realm of passive sampling. Because the model is able to accurately characterize the exchange kinetics for short timescales while also properly accounting for both polymer and boundary layer controls on diffusion, it can be used in combination with tracer release studies to infer characteristic boundary layer thickness of water bodies, which are important for understanding biogeochemical processes (e.g. diffusive fluxes from sediments, dissolution or nutrient uptake rates ${ }^{43}$ ).

\subsection{Conclusions}

In summary, the model presented in this paper successfully characterized the transport of various hydrophobic organic chemicals $\left(\log K_{O W}\right.$ 4.5-7.4) between $\mathrm{PE}$ and water in various turbulence conditions and in both finite and infinite bath conditions. The thickness of the boundary layer, the only input parameter that cannot be directly measured, can be understood as an indicator of the level of turbulence in the system, with $15-30 \mu \mathrm{m}$ for vigorous and 80-250 $\mu \mathrm{m}$ for gentle stirring. Though alternative models could be used once it is decided whether WBL or membrane control applies to a particular situation, our model (Equations 4.6-4.9, Code S1, Code S2) can be applied regardless of transfer regime (membrane-, mixed- or WBL-control). 
Compared to the commonly used sampling rate, this model is more accurate when partial or full membrane control is expected such as in high flow and/or when using thick or low diffusivity polymers. In addition, partial or full membrane control would also be applicable for chemicals with both low partition coefficients and low polymer diffusivities (e.g., pesticides or emerging contaminants with $\log K_{O W} 3-5$ and large molecular volumes). The finite bath model is particularly useful in interpretation of laboratory studies for the purpose of obtaining diffusion coefficients and partition coefficients. Because it can be used regardless of transfer regime, this model is accurate over a wider range of parameter values compared to the sampling rate formulation, and can therefore be used to investigate the relative importance of determinant parameters on PSDs/water equilibration times.

\section{Supplemental Information}

Text S1

Matlab codes S1-S2

Figures S1-S13

Table S1

\section{Acknowledgments}

Funding for this research was provided by ENI S.p.A (through the MIT Energy Initiative). We also acknowledge support from the Martin Family Society of Fellows for Sustainability (APT) and ESTCP contract W912HQ-09-C-0008 (PMG and JNA). We thank Prof. Charles Harvey for valuable input regarding Laplace transforms, Prof. Heidi Nepf for useful discussions, and Raffaella Borrelli and Pietro Cesti (ENI) for providing the $10 \mu \mathrm{m}$ PE and field work assistance. 


\section{References}

1. Muller, J. F.; Manomanii, K.; Mortimer, M. R.; McLachlan, M. S., Partitioning of polycyclic aromatic hydrocarbons in the polyethylene/water system. Fresen J Anal Chem 2001, 371, (6), 816-822.

2. Adams, R. G.; Lohmann, R.; Fernandez, L. A.; Macfarlane, J. K.; Gschwend, P. M., Polyethylene devices: Passive samplers for measuring dissolved hydrophobic organic compounds in aquatic environments. Environ Sci Technol 2007, 41, (4), 1317-1323.

3. Fernandez, L. A.; Lao, W.; Maruya, K. A.; White, C.; Burgess, R. M., Passive sampling to measure baseline dissolved persistent organic pollutant concentrations in the water column of the Palos Verdes Shelf Superfund site. Environ Sci Technol 2012, 46, (21), 11937-47.

4. Perron, M. M.; Burgess, R. M.; Suuberg, E. M.; Cantwell, M. G.; Pennell, K. G., Performance of passive samplers for monitoring estuarine water column concentrations: 1. Contaminants of concern. Environ Toxicol Chem 2013, 32, (10), 2182-2189.

5. Hawthorne, S. B.; Miller, D. J.; Grabanski, C. B., Measuring low picogram per liter concentrations of freely dissolved polychlorinated biphenyls in sediment pore water using passive sampling with polyoxymethylene. Anal Chem 2009, 81, (22), 9472-80.

6. Beckingham, B.; Ghosh, U., Polyoxymethylene passive samplers to monitor changes in bioavailability and flux of PCBs after activated carbon amendment to sediment in the field. Chemosphere 2013, 91, (10), 1401-1407.

7. Zeng, E. Y.; Tsukada, D.; Diehl, D. W., Development of solid-phase microextraction-based method for sampling of persistent chlorinated hydrocarbons in an urbanized coastal environment. Environ Sci Technol 2004, 38, (21), 5737-5743.

8. Rusina, T. P.; Smedes, F.; Klanova, J.; Booij, K.; Holoubek, I., Polymer selection for passive sampling: a comparison of critical properties. Chemosphere 2007, 68, (7), 1344-51.

9. Vrana, B.; Mills, G. A.; Allan, I. J.; Dominiak, E.; Svensson, K.; Knutsson, J.; Morrison, G.; Greenwood, R., Passive sampling techniques for monitoring pollutants in water. Trac Trend Anal Chem 2005, 24, (10), 845-868.

10. Sacks, V. P.; Lohmann, R., Development and use of polyethylene passive samplers to detect triclosans and alkylphenols in an urban estuary. Environ Sci Technol 2011, 45, (6), $2270-$ 7.

11. Moschet, C.; Vermeirssen, E. L. M.; Seiz, R.; Pfefferli, H.; Hollender, J., Picogram per liter detections of pyrethroids and organophosphates in surface waters using passive sampling. Water Research 2014, 66, 411-422.

12. Brown, R. S.; Akhtar, P.; Akerman, J.; Hampel, L.; Kozin, I. S.; Villerius, L. A.; Klamer, H. J. C., Partition controlled delivery of hydrophobic substances in toxicity tests using poly(dimethylsiloxane) (PDMS) films. Environ Sci Technol 2001, 35, (20), 4097-4102.

13. Smith, K. E. C.; Dom, N.; Blust, R.; Mayer, P., Controlling and maintaining exposure of hydrophobic organic compounds in aquatic toxicity tests by passive dosing. Aquatic Toxicol 2010, 98, (1), 15-24.

14. Booij, K.; Sleiderink, H. M.; Smedes, F., Calibrating the uptake kinetics of semipermeable membrane devices using exposure standards. Environ Toxicol Chem 1998, 17, (7), 1236-1245.

15. Booij, K.; Hofmans, H. E.; Fischer, C. V.; Van Weerlee, E. M., Temperature-dependent uptake rates of nonpolar organic compounds by semipermeable membrane devices and lowdensity polyethylene membranes. Environ Sci Technol 2003, 37, (2), 361-366. 
16. Rusina, T. P.; Smedes, F.; Koblizkova, M.; Klanova, J., Calibration of silicone rubber passive samplers: experimental and modeled relations between sampling rate and compound properties. Environ Sci Technol 2010, 44, (1), 362-7.

17. Crank, J., The mathematics of diffusion. 2nd ed.; Oxford University Press: Oxford, 1975; p 44-68.

18. Lohmann, R., Critical review of low-density polyethylene's partitioning and diffusion coefficients for trace organic contaminants and implications for its use as a passive sampler. Environ Sci Technol 2012, 46, (2), 606-18.

19. Gale, R. W., Three-compartment model for contaminant accumulation by semipermeable membrane devices. Environ Sci Technol 1998, 32, (15), 2292-2300.

20. Vrana, B.; Schuurmann, G., Calibrating the uptake kinetics of semipermeable membrane devices in water: Impact of hydrodynamics. Environ Sci Technol 2002, 36, (2), 290-296.

21. ter Laak, T. L.; Busser, F. J. M.; Hermens, J. L. M., Poly(dimethylsiloxane) as passive sampler material for hydrophobic chemicals: Effect of chemical properties and sampler characteristics on partitioning and equilibration times. Anal Chem 2008, 80, (10), 3859-3866.

22. Bayen, S.; Ter Laak, T. L.; Buffle, J.; Hermens, J. L., Dynamic exposure of organisms and passive samplers to hydrophobic chemicals. Environ Sci Technol 2009, 43, (7), 2206-15.

23. Booij, K.; Vrana, B.; Huckins, J. N., Theory, modelling and calibration of passive samplers used in water monitoring. In Passive Sampling Techniques in Environmental Monitoring, Greenwood, R.; Mills, G.; Vrana, B., Eds. Elsevier Science Bv: Amsterdam, 2007; Vol. 48, pp 141169.

24. Lohmann, R.; Booij, K.; Smedes, F.; Vrana, B., Use of passive sampling devices for monitoring and compliance checking of POP concentrations in water. Environ Sci Pollut $R$ 2012, $19,(6), 1885-1895$.

25. Reynolds, G. W.; Hoff, J. T.; Gillham, R. W., Sampling bias caused by materials used to monitor halocarbons in groundwater. Environ Sci Technol 1990, 24, (1), 135-142.

26. Hong, L.; Luthy, R. G., Uptake of PAHs into polyoxymethylene and application to oil-soot (lampblack)-impacted soil samples. Chemosphere 2008, 72, (2), 272-281.

27. Adams, R. G. Polyethylene devices and the effects of sediment resuspension on the cycling of PAHs and PCBs in the lower Hudson Estuary. PhD Thesis, Massachusetts Institute of Technology, 2003.

28. Hollenbeck, K. J. INVLAP.M: A matlab function for numerical inversion of Laplace transforms by the de Hoog algorithm http://www.isva.dtu.dk/staff/karl/invlap.htm

29. Thompson, J. M.; Hsieh, C. H.; Luthy, R. G., Modeling uptake of hydrophobic organic contaminants into polyethylene passive samplers. Environ Sci Technol 2015, 49, (4), 2270-7.

30. Mathias, S. A.; Zimmerman, R. W., Laplace transform inversion for late-time behavior of groundwater flow problems. Water Resour Res 2003, 39, (10), 1283.

31. Yeh, H. D.; Wang, C. T., Large-time solutions for groundwater flow problems using the relationship of small p versus large t. Water Resour Res 2007, 43, (6).

32. Booij, K.; Smedes, F.; van Weerlee, E. M., Spiking of performance reference compounds in low density polyethylene and silicone passive water samplers. Chemosphere 2002, 46, (8), 1157-1161.

33. Kundu, P. K.; Cohen, I. M., Fuild Mechanics. 2nd ed.; Elsevier Science: Academic Press, California, USA, 2002; p 531. 
34. Choi, Y.; Cho, Y. M.; Luthy, R. G., Polyethylene-water partitioning coefficients for parentand alkylated-polycyclic aromatic hydrocarbons and polychlorinated biphenyls. Environ Sci Technol 2013, 47, (13), 6943-50.

35. Hale, S. E.; Martin, T. J.; Goss, K.-U.; Arp, H. P. H.; Werner, D., Partitioning of organochlorine pesticides from water to polyethylene passive samplers. Environ Pollut 2010, 158, (7), 2511-2517.

36. Boudreau, B. P., The Benthic Boundary Layer: Transport Processes and Biogeochemistry. Oxford University Press: 2001; p 107-109.

37. Schwarzenbach, R. P.; Gschwend, P. M.; Imboden, D. M., Environmental Organic Chemistry. 2nd ed.; John Wiley: New York, 2003.

38. US Environmental Protection Agency SPARC http://archemcalc.com/sparc-web/calc /multiproperty (acessed June 2014)

39. Vrana, B.; Vermeirssen, E. L. M.; Allan, I. J.; Kohoutek, J.; Kennedy, K.; Mills, G. A.; Greenwood, R. Passive sampling of emerging pollutants in the aquatic environment: state of the art and perspectives. Position Paper. http://www.normannetwork.net/sites/default/files/norman_position_paper_pas_sampling.pdf

40. Booij, K.; Smedes, F., An improved method for estimating in situ sampling rates of nonpolar passive samplers. Environ Sci Technol 2010, 44, (17), 6789-94.

41. Morgan, E. J.; Lohmann, R., Detecting air-water and surface-deep water gradients of PCBs using polyethylene passive samplers. Environ Sci Technol 2008, 42, (19), 7248-7253.

42. Kot, A.; Zabiegała, B.; Namieśnik, J., Passive sampling for long-term monitoring of organic pollutants in water. Trac Trend Anal Chem 2000, 19, (7), 446-459.

43. Rominger, J. T.; Nepf, H. M., Effects of blade flexural rigidity on drag force and mass transfer rates in model blades. Limnol Oceanogr 2014, 59, (6), 2028-2041. 


\section{Supporting Information}

\section{Text S1. Derivation of analytical solution for passive samplers in water}

\subsection{General Procedure.}

In deriving all the analytical solutions presented in this manuscript, the following procedure is followed:

1. Write governing equations for concentration as function of time and distance, and any associated boundary and initial conditions.

2. Express all of the above in non-dimensional variables. Normalize distance by half thickness of $\mathrm{PE}$, time by $\mathrm{D}_{\mathrm{PE}} / \mathrm{L}^{2}$ and concentration by total concentration in the system (finite bath) and by the concentration at equilibrium (infinite bath).

3. Take Laplace transform of differential equations and boundary conditions. At this point the initial conditions are incorporated in the Laplace transform, and the time variable disappears.

4. Find solutions that satisfy the equations and use boundary conditions to determine the integration constants.

5. Typically the bulk concentration in the PE is of interest, so we take the integral of the solution across the thickness of PE.

6. Plug expression into a programing language or mathematical toolbox, such as Matlab and use an inversion algorithm to evaluate the solution in time domain.

\subsection{Derivation of solution for finite bath}

Sorption experiments with polymers in finite water baths are most often performed with all the chemical compound present in the water phase at $\mathrm{t}=0$. Therefore here we derive the solution describing the concentration in $\mathrm{PE}$ and water as a function of time, given the initial condition that $\mathrm{C}_{\mathrm{PE}}{ }^{0}=0$, and we only present the solution for the case where $\mathrm{C}_{\mathrm{W}}{ }^{0}=0$ at the end. We keep the same notations as in the main text, where $\delta$ is the thickness of the boundary layer.

The governing equations for the finite bath case are the following:

$$
\begin{array}{ll}
\frac{\partial C_{P E}}{d t}=D_{P E} \frac{\partial^{2} C_{P E}}{\partial x^{2}} & \text { for }-L<x<L \\
\frac{\partial C_{W}}{d t}=D_{w} \frac{\partial^{2} C_{W}}{\partial x^{2}} & \text { for } L<x<L+\delta \text { and }-\delta-L<x<-L
\end{array}
$$

and they are subject to the following boundary conditions:

- No flux at the center of sampler:

$$
\frac{\partial C_{P E}}{\partial X}=0 \text { at } x=0 \text {; }
$$


- Chemical equilibrium at sampler/water interface:

$C_{P E}=K_{P E W} C_{W}$ at $x= \pm L$

- No accumulation of mass at sampler-water interface

$$
D_{P E} \frac{\partial C_{P E}}{\partial X}=D_{W} \frac{\partial C_{W}}{\partial X} \text { at } x= \pm L
$$

- Flux out of PE controls the concentration in the water over time:

$$
\frac{\partial C_{W}}{d t}=-D_{W} \frac{A_{P E}}{V_{W}} \frac{\partial C_{W}}{\partial x} \text { for } x= \pm(L+\delta)
$$

and initial conditions:

$$
\begin{aligned}
& C_{W}=C_{W}^{0} \text { at } x>L \text { and } x<-L \ldots \ldots \ldots \\
& C_{P E}=C_{P E}^{0}=0 \text { at } x<L \text { and } x>-L
\end{aligned}
$$

The first step in deriving the solution is to transform the equations and boundary conditions in non-dimensional units. $\bar{C}, X, T$ are now unit-less variables for concentration, distance and time:

$$
\begin{aligned}
& \overline{C_{P E}}=\frac{C_{P E}}{C_{W}^{0} \frac{V W}{m_{P E}} \rho_{P E}} \text { and } \overline{C_{W}}=\frac{C_{W}}{C_{W}^{0}} \\
& X=\frac{x}{L} \text { and } T=t \frac{D_{P E}}{L^{2}}
\end{aligned}
$$

where $\mathrm{L}$ is the half thickness of the polymer, $D_{P E}$ is the diffusivity in the PE and $K_{P E W}$ is the PEwater partition coefficient, $\mathrm{V}_{\mathrm{W}}$ is the volume of water, $\mathrm{m}_{\mathrm{PE}}$ and $\rho_{P E}$ are the mass and density of $\mathrm{PE}$, respectively. In non-dimensional terms the initial conditions for $\mathrm{T}=0$ are:

$$
\begin{aligned}
& \overline{C_{W}^{0}}=1 \text { for } X<-1 \text { and } X>1 \\
& \overline{C_{P E}^{0}}=0 \text { for }-1<X<1 \ldots \ldots \ldots
\end{aligned}
$$

Similarly, at $\mathrm{T}>0$, we have the following boundary conditions in non dimensional units:

- No flux at the center of sampler:

$$
\frac{\partial \overline{C_{P E}}}{\partial X}=0 \text { at } X=0 ;
$$

- Chemical equilibrium at sampler/water interface:

$$
\overline{C_{P E}}=\frac{L}{H} K_{P E W} \overline{C_{W}} \text { at } X= \pm 1
$$

- No accumulation of mass at sampler-water interface

$$
\frac{\partial \overline{C_{P E}}}{\partial X}=\frac{L}{H} \psi \frac{\partial \overline{C_{W}}}{\partial X} \text { at } X= \pm 1 \text {, where } \psi=\frac{D_{W}}{D_{P E}}
$$

- Flux out of PE controls the concentration in the water over time:

$$
\frac{\partial \overline{C_{W}}}{d T}=-\frac{L}{H} \psi \frac{\partial \overline{C_{W}}}{\partial X} \text { for } x= \pm(1+\alpha)
$$

where $\mathrm{H}$ is the ratio of volume of water to area of $\mathrm{PE}, \alpha=\delta / \mathrm{L}$. Lastly, we perform the same change of variables for the governing equations:

$$
\begin{aligned}
& \frac{\partial^{2} \overline{C_{P E}}}{\partial X^{2}}=\frac{\partial \overline{C_{P E}}}{\partial T} . \\
& \frac{\partial^{2} \overline{C_{W}}}{\partial X^{2}}=\frac{1}{\psi} \frac{\partial \overline{C_{W}}}{\partial T} .
\end{aligned}
$$

By taking Laplace transforms of the governing equations, we translate the equations from time 
domain to complex s domain, and thus reduce the complexity of the differential equations. $\widehat{C_{P E}}$ and $\widehat{C_{W}}$ denote the Laplace domain expression of concentration. We now have the following governing equations and boundary conditions that are independent of the time variable:

- governing equations:

$$
\begin{aligned}
& \frac{\partial^{2} \widehat{C_{P E}}}{\partial X^{2}}=s \widehat{C_{P E}} ; \ldots \\
& \frac{\partial^{2} \widehat{C_{W}}}{\partial X^{2}}=\frac{s}{\psi} \widehat{C_{W}}-\frac{1}{\psi} ;
\end{aligned}
$$

- boundary conditions:

$$
\begin{aligned}
& \frac{\partial \widehat{C_{P E}}}{\partial X}=0 \text { at } X=0 \\
& \widehat{C_{P E}}=\frac{L}{H} K_{P E W} \widehat{C_{W}} \text { at } X=1 \\
& \frac{\partial \widehat{C_{P E}}}{\partial X}=\frac{L}{H} \psi \frac{\partial \widehat{C_{W}}}{\partial X} \text { at } X=1 \\
& \frac{\partial \widehat{C_{W}}}{\partial X}=-\frac{s}{\psi} \frac{H}{L} \widehat{C_{W}}+\frac{1}{\psi} \frac{H}{L} \text { at } X=1+\alpha
\end{aligned}
$$

The solutions found to satisfy both governing equations (Equations 19 and 20) and the first boundary condition (Equation 21) above:

$$
\begin{aligned}
& \widehat{C_{P E}}=A \cosh (X \sqrt{s}) \\
& \widehat{C_{W}}=\frac{1}{s}+B \sinh \left(\sqrt{\frac{s}{\psi}}(X-1-\alpha)\right)+C \cosh \left(\sqrt{\frac{s}{\psi}}(X-1-\alpha)\right)
\end{aligned}
$$

Using the remaining 3 boundary conditions (Equations 22-24), we can calculate the values of A $\mathrm{B}$ and $\mathrm{C}$ :

$$
\begin{aligned}
& A=\frac{1}{s} \frac{K_{P E W} \frac{L}{H}}{\cosh (\sqrt{s})-\sinh \sqrt{s} \frac{K_{P E W}}{\sqrt{\psi}}\left(\frac{1-\sqrt{\frac{\psi}{S} H} \operatorname{coth}\left(-\alpha \sqrt{\frac{s}{\psi}}\right)}{\operatorname{coth}\left(-\alpha \sqrt{\frac{s}{\psi}}\right)-\frac{L}{H} \sqrt{\frac{\psi}{s}}}\right)} . \\
& B=\frac{D \sinh \sqrt{s}}{\frac{L}{H} \sqrt{\psi}\left(\cosh \left(-\alpha \sqrt{\frac{s}{\psi}}\right)-\sqrt{\left.\frac{\bar{\psi}}{s H} \sinh \left(-\alpha \sqrt{\frac{s}{\psi}}\right)\right)}\right.} \\
& C=-B \sqrt{\frac{\psi}{S}} \frac{L}{H}
\end{aligned}
$$

Lastly, we plug (27) into (25) evaluate the average normalized concentration in the PE using:

$$
\widehat{M_{P E}}=\int_{0}^{1} \widehat{C_{P E}} d X
$$

Adjusting to absolute concentrations (as opposed to normalized) using Equation 9, we obtain the following expression in Laplace space for the concentration in PE:

$$
C_{P E}=C_{W}^{0} \frac{1}{s^{\frac{3}{2}}} \frac{K_{P E W}}{\operatorname{coth}(\sqrt{s})-\frac{K_{P E W}\left[1-\frac{L}{H} \sqrt{\frac{\psi}{s}} \operatorname{coth}\left(-\alpha \sqrt{\frac{s}{\psi}}\right)\right]}{\sqrt{\psi}\left[\operatorname{coth}\left(-\alpha \sqrt{\frac{s}{\psi}}\right)-\frac{L}{H} \sqrt{\frac{\psi}{s}}\right]}}
$$

For evaluating the concentration in the water phases, we plug (28) and (29) into (26) and evaluate $\widehat{C_{W}}$ at $X=1+\alpha$, the edge of the boundary layer: 


$$
C_{W}=C_{W}^{0}\left(\frac{1}{S}-\frac{1}{s^{\frac{3}{2}}} \frac{K_{P E W} \frac{L}{H}}{\operatorname{coth}(\sqrt{s})-\frac{K_{P E W}\left[1-\frac{L}{H} \sqrt{\frac{\psi}{S}} \operatorname{coth}\left(-\alpha \sqrt{\frac{s}{\psi}}\right)\right]}{\sqrt{\psi}\left[\operatorname{coth}\left(-\alpha \sqrt{\frac{s}{\psi}}\right)-\frac{L}{H} \sqrt{\frac{\psi}{s}}\right]}} \frac{1}{\left[\cosh \left(-\alpha \sqrt{\frac{s}{\psi}}\right)-\frac{L}{H} \sqrt{\frac{\psi}{S}} \sinh \left(-\alpha \sqrt{\frac{s}{\psi}}\right)\right]}\right)
$$

Equations 31 and 32 cannot be inverted to time domain using inversion tables. Instead we use a numerical inversion algorithm in MATLAB invlap.m ${ }^{1}$, to find the corresponding mass of chemical in water and PE as function of time T. A sample code to perform this operation is provided bellow (Code S1).

If the chemical is present only in the PE at time zero, the normalization of concentration in PE and water has to be done by dividing by $C_{P E}^{0}$ (derivation not shown here). Following the same mathematical procedure, the concentration of chemical in PE and water in Laplace domain are given by:

$$
\begin{aligned}
& C_{P E}=C_{P E}^{0}\left(\frac{1}{s}+\frac{1}{s^{\frac{3}{2}}} \frac{1}{\frac{K_{P E W}\left[1-\frac{L}{H} \sqrt{\frac{\psi}{s}} \operatorname{coth}\left(-\alpha \sqrt{\frac{s}{\psi}}\right)\right]}{\sqrt{\psi}\left[\operatorname{coth}\left(-\alpha \sqrt{\frac{s}{\psi}}\right)-\frac{L}{H} \sqrt{\frac{\psi}{s}}\right]}-\operatorname{coth}(\sqrt{s})}\right) \\
& C_{W}=-C_{P E}^{0} \frac{L}{H} \frac{1}{S^{\frac{3}{2}}} \frac{1}{\operatorname{coth}(\sqrt{s})-\frac{K_{P E W}\left[1-\frac{L}{H} \sqrt{\frac{\psi}{s}} \operatorname{coth}\left(-\alpha \sqrt{\frac{s}{\psi}}\right)\right]}{\sqrt{\psi}\left[\operatorname{coth}\left(-\alpha \sqrt{\frac{s}{\psi}}\right)-\frac{L}{H} \sqrt{\frac{\psi}{s}}\right]}} \frac{1}{\left[\cosh \left(-\alpha \sqrt{\frac{s}{\psi}}\right)-\frac{L}{H} \sqrt{\frac{\psi}{S}} \sinh \left(-\alpha \sqrt{\frac{s}{\psi}}\right)\right]}
\end{aligned}
$$

\subsection{Late time approximation for finite bath solution.}

The expressions for the evolution of the mass of compound inside of PE can be simplified by using the Laurent series approximations of the hyperbolic cotangent, mentioned previously, and those of hyperbolic sine and cosine:

$$
\begin{aligned}
& \cosh x=1+\frac{1}{2} x^{2}-\frac{1}{24} x^{4}+\frac{1}{720} x^{6}-\frac{1}{40320} x^{8}+\cdots \\
& \sinh x=x+\frac{1}{6} x^{3}+\frac{1}{120} x^{5}+\frac{1}{5040} x^{7}+\frac{69}{362880} x^{9}+\cdots
\end{aligned}
$$

Using the first three terms of each series, and then neglecting the $s^{3}$ terms for small values of $\mathrm{s}$, we obtain the following expression for $\widehat{C_{P E}}$ :

$$
\widehat{C_{P E}}=\frac{L}{H} K_{P E W} \frac{\left(\frac{1}{\alpha}+\frac{L}{H \psi}\right)+\frac{1 \alpha}{3 \psi} s}{\left(\frac{1}{\alpha}+\frac{L}{H}+K_{P E W} \frac{L 1}{H \alpha}\right) s+\left(\frac{\alpha}{3 \psi}+\frac{1}{\alpha}+\frac{1 L}{3 H}+\frac{K_{P E W}}{\psi}\right) s^{2}}
$$

Using inversion tables, find the following expression for $C_{P E}$ in time domain:

$$
\frac{C_{P E}}{C_{W}^{0}}=\left[\frac{\left(\frac{1}{\alpha}+\frac{L}{H \psi}\right) K_{P E W}}{\frac{1}{\alpha}+\frac{L}{H}+K_{P E W} \frac{L 1}{H \alpha}}+\left(\frac{\frac{\alpha}{\psi} K_{P E W}}{\frac{\alpha}{3 \psi}+\frac{1}{3 \alpha}+\frac{L}{3 H}+\frac{K_{P E W}}{\psi}}-\frac{\left(\frac{1}{\alpha}+\frac{L}{H \psi}\right) K_{P E W}}{\frac{1}{\alpha}+\frac{L}{H}+\frac{K_{P E W} L}{\alpha}}\right) e^{-\frac{\frac{1}{\alpha}+\frac{L}{H}+K_{P E W} \frac{L}{H \alpha}}{3 \psi}+\frac{1}{3 \alpha}+\frac{L}{3 H}+\frac{K_{P E W}}{\psi}}\right]
$$

where $C_{W}^{0}$ in this expression is the concentration at time zero in the water. Though rather complicated, this expression can be evaluated without Matlab. However, due to the complexity 
of this expression, and the fact that it is an approximation, we recommend that the full solution should be used whenever possible.

\subsection{Existing finite bath models}

For convenience, we also reproduce here the solution for the diffusion of a chemical into a polymer sheet from a finite bath well mixed solution (Equation 4.37 in Crank, 1975, ref $^{2}$ ), in notation used above:

$$
\frac{C_{P E}}{C_{W}^{0}}=\frac{K_{P E W}}{1+\frac{K_{P E W} L}{H}}\left(1-\sum_{n=1}^{\infty} \frac{\frac{2 H}{L K_{P E W}}\left(1+\frac{H}{L K_{P E W}}\right)}{1+\frac{H}{L K_{P E W}}+\frac{H^{2}}{L^{2} K_{P E W}^{2}} q_{n}^{2}} e^{-\frac{D_{P E} q_{n}^{2} t}{L^{2}}}\right)
$$

where $q_{n}$ are the roots of:

$$
\tan q_{n}=-\frac{H}{L K_{P E W}} q_{n}
$$

Also reproduced here is the equation derived by Booij (2007, ref $^{3}$, Equation 7.39), for the uptake of chemicals into polymer sheets from finite bath solutions, given as a function of sampling rate (the volume of water per time cleared by the polymer), and keeping all other notations consistent:

$$
\frac{C_{P E}}{C_{W}^{0}}=\frac{K_{P E W}}{1+\frac{K_{P E W} L}{H}}\left(1-e^{-\frac{\left(1+\frac{K_{P E W} L}{H}\right) R_{S} t}{V_{S} K_{P E W}}}\right)
$$

Comparison of Equation 39 and 41 reveals that are both equal as $t \rightarrow \infty$, but the rate of approach to equilibrium is different. Equation 39 has a pre-exponential factor that is system dependent (depends on $q_{n}$ which depends on the phase ratio and the partition coefficient $\mathrm{K}$ according to Equation 40). In contrast, for Equation 41 the pre-exponential factor is 1, which means that the sampling rate equation 1-exp(-kt) should not be used to determine sampling rates in membrane controlled regimes, as the fitted coefficients are not going to reflect the true membrane resistance (as it would if we were to fit Equation 39 instead).

\subsection{Infinite bath solution}

We can derive the corresponding infinite bath solution from the finite bath case by taking the limit as $\mathrm{V}_{\mathrm{W}} \rightarrow \infty$. With an infinite water volume, Equation 6 becomes $\mathrm{dC}_{\mathrm{w}} / \mathrm{dt}=0$, implying that the concentration in the water outside of the WBL does not change with time. Imposing that $\mathrm{C}_{\mathrm{w}}$ does not change with time in infinite bath scenarios makes sense since the sampler typically does not have enough capacity to change the environmental concentration of target compounds or PRCs. All other governing equations (Equations 1-2), boundary conditions (3-5) and initial conditions (7-8) remain the same for infinite versus finite bath conditions. Thus, as the infinite bath is simply a particular case of the more general finite bath, we can derive the corresponding solutions from the finite bath solutions (Equations 31-34) by taking the limit as $V_{W} \rightarrow \infty$. It follows then that, using Equation 33, the fractional PRC remaining in PE after a certain time is given by: 


$$
f_{P R C}=\lim _{H \rightarrow \infty} \frac{C_{P E}}{C_{P E}^{0}}=\lim _{H \rightarrow \infty}\left(\frac{1}{s}+\frac{1}{s^{\frac{3}{2}}} \frac{1}{\frac{K_{P E W}\left[1-\frac{L}{H} \sqrt{\frac{\psi}{s}} \operatorname{coth}\left(-\alpha \sqrt{\frac{s}{\psi}}\right)\right]}{\sqrt{\psi}\left[\operatorname{coth}\left(-\alpha \sqrt{\frac{s}{\psi}}\right)-\frac{L}{H} \sqrt{\frac{\psi}{S}}\right]}-\operatorname{coth}(\sqrt{s})}\right)
$$

which leads to:

$$
f_{P R C}=\frac{1}{s}+\frac{1}{s^{\frac{3}{2}}} \frac{1}{\frac{\mathrm{K}_{\mathrm{PEW}}}{\sqrt{\psi}} \tanh \left(-\alpha \sqrt{\frac{s}{\psi}}\right)-\operatorname{coth}(\sqrt{s})}
$$

For target chemicals diffusing into $\mathrm{PE}\left(\mathrm{C}^{0}{ }_{\text {target,PE }}=0\right.$ and $\left.\mathrm{C}_{\mathrm{W} \text {,target }}=\mathrm{C}^{0}{ }_{\mathrm{w} \text {,target }}\right)$ the corresponding solution for the accumulation in $\mathrm{PE}$ can be obtained using Equation 31:

$$
\begin{aligned}
& f_{\text {target }}=\lim _{H \rightarrow \infty} \frac{C_{P E}}{C_{W}^{0} K_{P E W}}=\lim _{H \rightarrow \infty} \frac{1}{s^{\frac{3}{2}}} \frac{1}{\operatorname{coth}(\sqrt{s})-\frac{K_{P E W}\left[1-\frac{L}{H} \sqrt{\frac{\psi}{s}} \operatorname{coth}\left(-\alpha \sqrt{\frac{s}{\psi}}\right)\right]}{\sqrt{\psi}\left[\operatorname{coth}\left(-\alpha \sqrt{\frac{s}{\psi}}\right)-\frac{L}{H} \sqrt{\frac{\psi}{s}}\right.}} \\
& f_{\text {target }}=\frac{C_{P E}}{C_{P E}^{\infty}}=\frac{1}{s^{3 / 2}} \frac{1}{\operatorname{coth}(\sqrt{s})-\frac{K_{\mathrm{PEW}}}{\sqrt{\psi}} \tanh \left(-\alpha \sqrt{\frac{s}{\psi}}\right)}
\end{aligned}
$$

As expected the amount of PRC left in the PE is related to the amount of target diffused into the PE by (Laplace transform of 1 is $1 / \mathrm{s}$ ):

$$
f_{\text {target }}=\frac{1}{s}-f_{P R C}
$$

Also, for $\alpha=0$ (no aqueous boundary layer) the solution for uptake into PE from infinite bath (Equation 45) is in agreement with Eq 2.52 (Crank, 1975, ref $^{2}$ ), the Laplace domain solution for diffusion in an plane sheet from an infinite bath, reproduced here in the notation used in the derivation above, and integrated across the membrane half thickness:

$$
f_{\text {target }}=\frac{1}{s^{\frac{3}{2}} \operatorname{coth} \sqrt{s}}
$$

We note that one arrives at the same solution for the infinite bath by following through the steps outlined in S1.1 General Procedures, and starting with the governing equations, boundary and initial conditions outlined at the beginning of this sections.

Expressions 43 and 45 cannot be inverted to time domain using inversion tables. Instead we use a MATLAB numerical inversion algorithm invlap. ${ }^{1}$ to find the corresponding mass of PRC left in the PE as function of time T. A sample code to perform this operation is provided in Code S2.

\subsection{Late time approximations for infinite bath solutions}

The expressions for the evolution of the mass of compound inside of PE can be simplified by using the Laurent series approximations of the hyperbolic tangent and cotangent:

$$
\begin{aligned}
& \operatorname{coth} x=x^{-1}+\frac{1}{3} x-\frac{1}{45} x^{3}+\frac{2}{945} x^{5}-\frac{1}{4725} x^{7}+\cdots \\
& \tanh x=x-\frac{1}{3} x^{3}+\frac{2}{15} x^{5}-\frac{17}{315} x^{7}+\frac{69}{2835} x^{9}+\cdots
\end{aligned}
$$


Using the first three terms of each series we obtain the following expression for fraction of target taken up by PE:

$$
f_{\text {target }}=\frac{1}{s+\left(\frac{1}{3}+\frac{K_{P E W^{\alpha}}}{\psi}\right) s^{2}-\left(\frac{1}{45}+\frac{1 K_{P E W}}{3 \psi^{2}}\right) s^{3}+\frac{2 K_{P E W} \psi^{3}}{15} \alpha^{5} s^{4}} .
$$

For small values of $\mathrm{s}$, we can neglect $s^{3}$ and $s^{4}$ terms, and using inversion tables, find the following expression for $C_{\text {target }}$ :

$$
C_{\text {target }}=C_{\text {target }}^{\infty}\left(1-e^{\frac{-\frac{t}{\frac{K_{P E W} L}{D_{W}}+\frac{L^{2}}{3 D_{P E}}}}{{ }^{2}}}\right.
$$

where $C_{\text {target }}$ in this expression is the concentration of target analyte in the PE, integrated over the thickness of the sampler, and $C_{\text {target }}^{\infty}$ is the concentration at equilibrium with the well mixed bath:

$$
C_{\text {target }}^{\infty}=C_{w}^{0} K_{P E W}
$$

Similarly for the concentration of a PRC over time we obtain:

$$
C_{P R C}=C_{P R C}^{0} e^{-\frac{t}{\frac{K_{P E W} L \delta}{D_{W}}+\frac{L^{2}}{3 D_{P E}}}}
$$

\subsection{Existing infinite bath models}

For comparison, the diffusion into a plane sheet from an infinite bath solution (Equation 4.42 from Crank, 1975, ref $^{2}$ ), is reproduced here for diffusion of a target compound into PE, and using the same notations as used in the derivation above:

$$
\frac{C_{\text {target }}}{C_{\text {target }}^{\infty}}=1-\sum_{n=0}^{\infty} \frac{8}{(2 n+1)^{2} \pi^{2}} e^{-\frac{D_{P E}\left(n+\frac{1}{2}\right)^{2} \pi^{2} t}{L^{2}}}
$$

For $\mathrm{n}=0$ the equation reduces to:

$$
C_{\text {target }}=C_{\text {target }}^{\infty}\left(1-\frac{8}{\pi^{2}} e^{-\frac{D_{P E} \pi^{2} t}{4 L^{2}}}\right)
$$

and Figure S13 shows that Equation 55 coincides with the full solution for timescales greater than $10 \%$ of the equilibration time 


\section{Code S1. Matlab routine for numerical inversion of finite bath solution.}

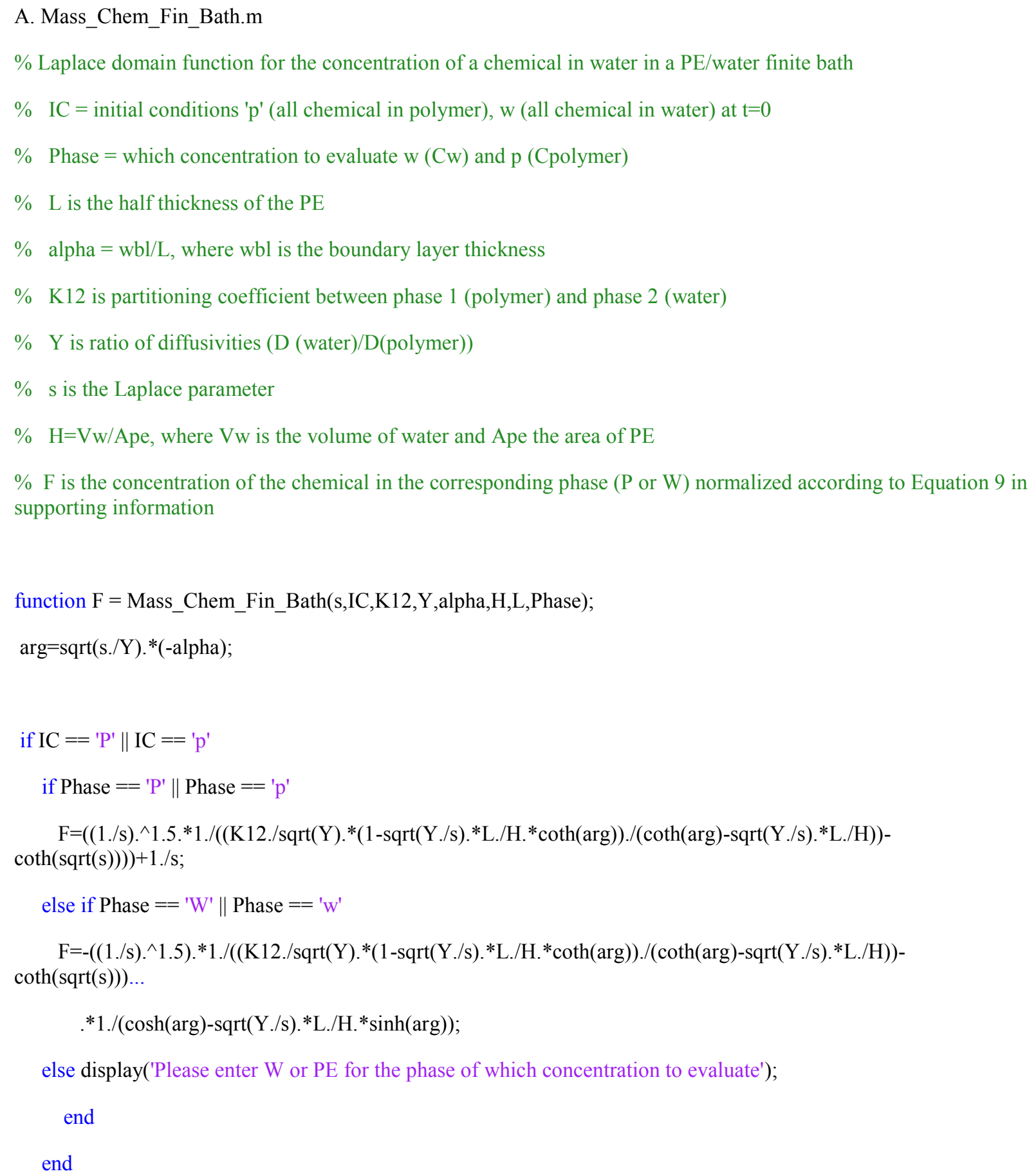


$\mathrm{F}=\mathrm{K} 12 .{ }^{*} \mathrm{~L} . / \mathrm{H} .{ }^{*}(1 . /(\mathrm{s} . \wedge(1.5))) . /\left(\operatorname{coth}(\operatorname{sqrt}(\mathrm{s}))-\mathrm{K} 12 . / \operatorname{sqrt}(\mathrm{Y}) .{ }^{*}\left(1-\operatorname{sqrt}(\mathrm{Y} . / \mathrm{s}) .{ }^{*} \mathrm{~L} . / \mathrm{H} .{ }^{*} \operatorname{coth}(\arg )\right) . /(\operatorname{coth}(\arg )-\right.$ $\operatorname{sqrt}(\mathrm{Y} . / \mathrm{s}) . * \mathrm{~L} . / \mathrm{H}))$;

else if Phase $==$ 'W' $\|$ Phase $==$ ' $\mathrm{W}$ '

$\mathrm{F}=(1 . / \mathrm{s})-\left(\mathrm{K} 12 .{ }^{*} \mathrm{~L} . / \mathrm{H}\right) .{ }^{*}\left(1 . /\left(\mathrm{s} .{ }^{\wedge}(1.5)\right)\right) . /\left(\cosh (\arg )-\mathrm{sqrt}(\mathrm{Y} . / \mathrm{s}) .{ }^{*} \mathrm{~L} . / \mathrm{H} .{ }^{*} \sinh (\arg )\right) .{ }^{*} 1 . /(\operatorname{coth}(\operatorname{sqrt}(\mathrm{s}))-$ K12./sqrt(Y).*(1-sqrt(Y./s).*L./H.*coth(arg))./(coth(arg)-sqrt(Y./s). ${ }^{*}$ L./H));

else disp('Please enter W or PE for the phase of which concentration to evaluate')

end

end

else disp('Please enter W or P for Initial conditioin variable, IC')

end

end

end

\section{B. ModelScript_Fin_Bath.m}

\%ModelScript_Fin_Bath.m

\% This script calculates the concentration of a chemicals over time in a

\%PE-water system of finite, and known dimensions.

\%This is an illustrative example with 5 compounds : PCB 28, 4,4 DDD, PCB 52, 4,4 DDE, PCB 153

\%This calculation needs the function invlap.m which can be downloaded at

\%www.cambridge.org/us/engineering/author/nellisandklein/downloads/invlap.m

clear all

\%define inputs

time $=10 ; \%$ days

$\mathrm{L}=12.75 * 10^{\wedge}-4 ; \%$ half thickness of polymer $(\mathrm{cm})$

wbl $=25 * 10^{\wedge}-4 ; \%$ boundary layer thickness $(\mathrm{cm})$

mpe $=15 ; \% \mathrm{mg}$

$\mathrm{Vw}=4000 ; \% \mathrm{~cm}^{\wedge} 3$

$\mathrm{IC}=$ 'W'; \%where is the chemical at $\mathrm{T}=0$ ? ' $\mathrm{P}$ ' polymer, ' $\mathrm{W}$ ' water 
\%define chemical properties

Comp_names=\{'4,4 DDD', 'PCB 28', 'PCB 47',' 4,4 DDE', 'PCB 153'\};

MW_array= [320 268.4 303.9 318 375];

Dpe_array $=10 .{ }^{\wedge}[-9.39-8.81-8.98-9.33-9.33] ; \% \mathrm{~cm}^{\wedge} 2 / \mathrm{s}$, calculated based on Rainer 2011

Kpew_array=10.^[4.86 5.22 5.57 5.76 6.77]; \%from Hale et al. 2010 and Choi et al. 2013

$\%$ evaluate other needed quantities

Dw_array $=\left(2.7 * 10^{\wedge}-4\right) . /\left(\mathrm{MW}\right.$ _array. $\left.{ }^{\wedge} 0.71\right) ; \%$ Diffusivity of compound in water $\left(\mathrm{cm}^{\wedge} 2 / \mathrm{s}\right)$

$\mathrm{H}=\mathrm{Vw} . /\left(\mathrm{mpe} / 0.95 * 10^{\wedge}-3 / \mathrm{L}\right) ; \% \mathrm{~cm}$

alpha $=\mathrm{wbl} / \mathrm{L}$;

$\%$ colors for plotting

linespec_array=['r' 'b' 'g' 'm' 'k']; \%matlab linespecs

$\%$ generate a time vector based on duration of deployment (time)

$\mathrm{t}=0.0001: .1$ :time; \%days

\%perform transport calculation on all congeners

for $\mathrm{n}=1$ :length(Comp_names)

$\mathrm{K} 12=\mathrm{Kpew}$ array(n);

$\mathrm{Y}=\left(\mathrm{Dw} \_\operatorname{array}(\mathrm{n}) / \mathrm{Dpe} \_\operatorname{array}(\mathrm{n})\right)$;

$\mathrm{T}=\mathrm{t}^{*} 3600 * 24 *$ Dpe_array $(\mathrm{n}) /\left(\mathrm{L}^{\wedge} 2\right)$;

for $\mathrm{i}=1$ :length(t)

Phase='w'; \%which concentration to evaluate $\mathrm{P}$ for polymer, W for water phase

M(i)=invlap('Mass_Chem_Fin_Bath', T(i),0,1e-9,IC,K12,Y,alpha,H,L,Phase); \% invlap.m (2,3)

$\%$ note this normalized concentration

$\%$ to calculate absolute concentration multiply by initial concentration in the units of the corresponding phase

$\%$ for example if $\mathrm{Cw} 0=1$ and one wants to evaluate $\mathrm{CPE}$ then $\mathrm{CPE}=\mathrm{M} * \mathrm{Cw} 0 *(\mathrm{Vw} / \mathrm{VPE})$ or $\mathrm{CPE}=\mathrm{M} * \mathrm{Cw} 0 * \mathrm{H} / \mathrm{L}$

end

\%plot

$\operatorname{plot}(\mathrm{t}, \mathrm{M}, \mathrm{linespec} \operatorname{array}(\mathrm{n}))$

hold on

end 


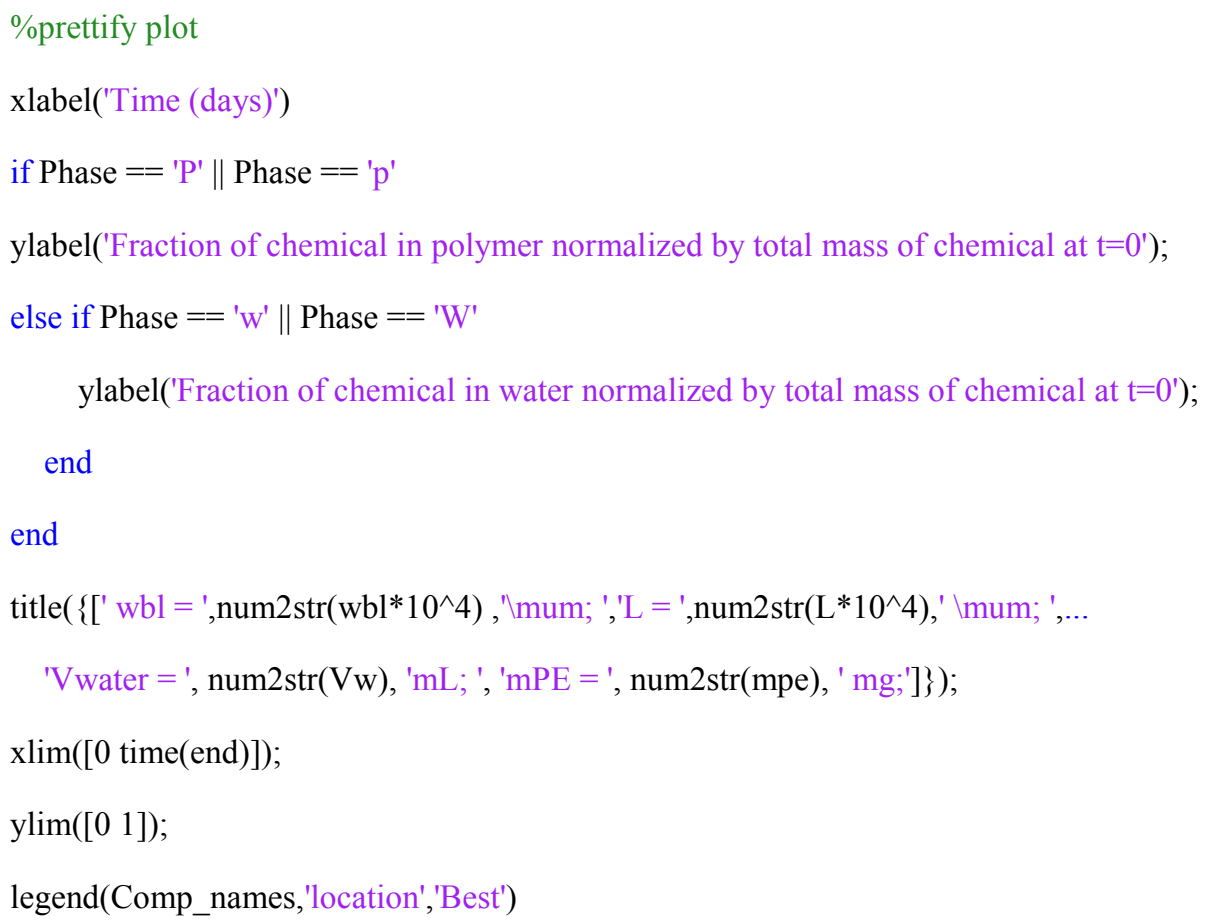

\section{Code S2. Matlab routine for numerical inversion of infinite bath solution.}

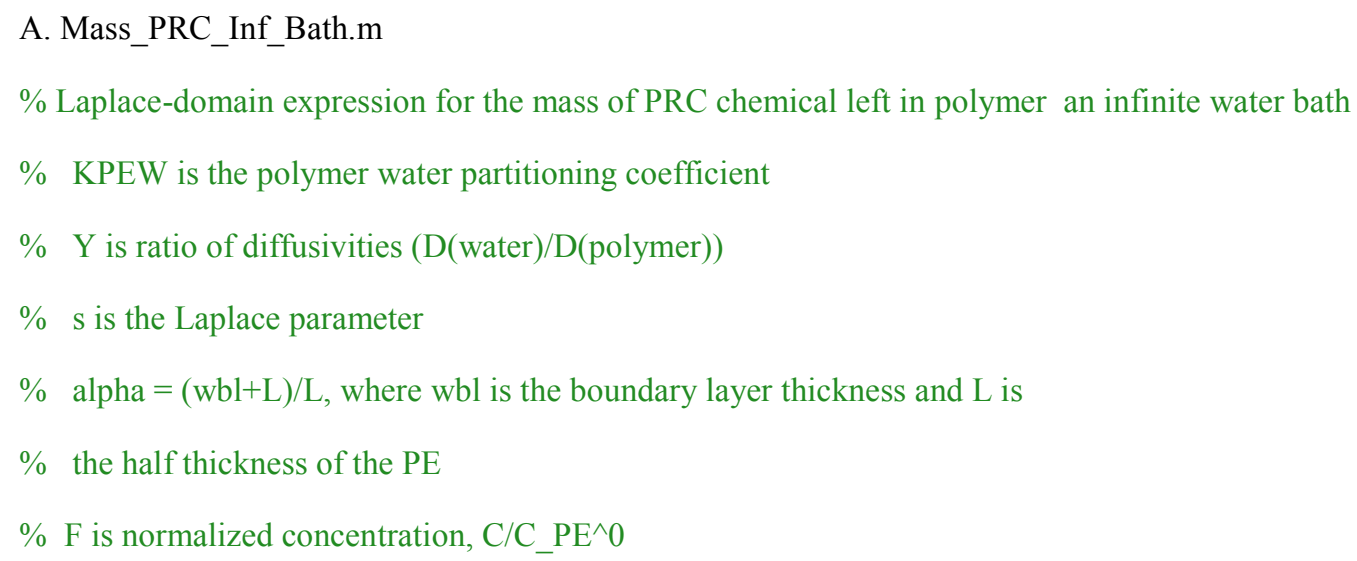


\%This script calculates the fraction of PRC left and target accumulated over time when PE is deployed in water

\%This is an illustrative example with 5 compounds : PCB 28, 4,4 DDD, PCB 52, 4,4 DDE, PCB 153

\%This calculation needs the function invlap.m which can be downloaded at

\%www.cambridge.org/us/engineering/author/nellisandklein/downloads/invlap.m

clear all

\%define inputs

time $=35 ; \%$ days

$\mathrm{L}=12.75 * 10^{\wedge}-4 ; \%$ half thickness of polymer $(\mathrm{cm})$

wbl $=200 * 10^{\wedge}-4 ; \%$ boundary layer thickness $(\mathrm{cm})$

\%define chemical properties

Comp_names=\{'4,4 DDD', 'PCB 28', 'PCB 47',' 4,4 DDE', 'PCB 153'\};

MW_array $=\left[\begin{array}{lll}320 & 268.4 & 303.9 \\ \hline & 318375\end{array}\right]$;

Dpe_array $=10 .^{\wedge}[-9.39-8.81-8.98-9.33-9.33] ; \% \mathrm{~cm}^{\wedge} 2 / \mathrm{s}$, calculated based on Rainer 2011

Kpew_array=10.^[4.86 5.22 5.57 5.76 6.77]; \%from Hale et al. 2010 and Choi et al. 2013, in LW/LPE units

$\%$ evaluate other needed quantities

Dw_array $=\left(2.7 * 10^{\wedge}-4\right) . /\left(\mathrm{MW}\right.$ array. $\left.{ }^{\wedge} 0.71\right) ; \%$ Diffusivity of compound in water $\left(\mathrm{cm}^{\wedge} 2 / \mathrm{s}\right)$

alpha $=\mathrm{wbl} / \mathrm{L}$;

$\%$ colors for plotting

linespec_array=['r' 'b' 'g' 'm' 'k']; \%matlab linespecs

$\%$ generate a time vector based on duration of deployment (time)

$\mathrm{t}=0.1: 1:$ time; $\%$ days

figure(1);

Transport_PRC=gca;

xlabel('Time(days)');

ylabel('Amount of PRC left in PE normalized to $\mathrm{t}=0$ value, $\mathrm{C} / \mathrm{Co}$ ');

title(['Infinite Bath Model' ' wbl = ',num2str(wbl*10^4) ,'micrometers; ',' PE thickness = ',num2str(2*L*10^4),' micrometers; ',...

]);

$x \lim ([0$ time(end) $])$; 


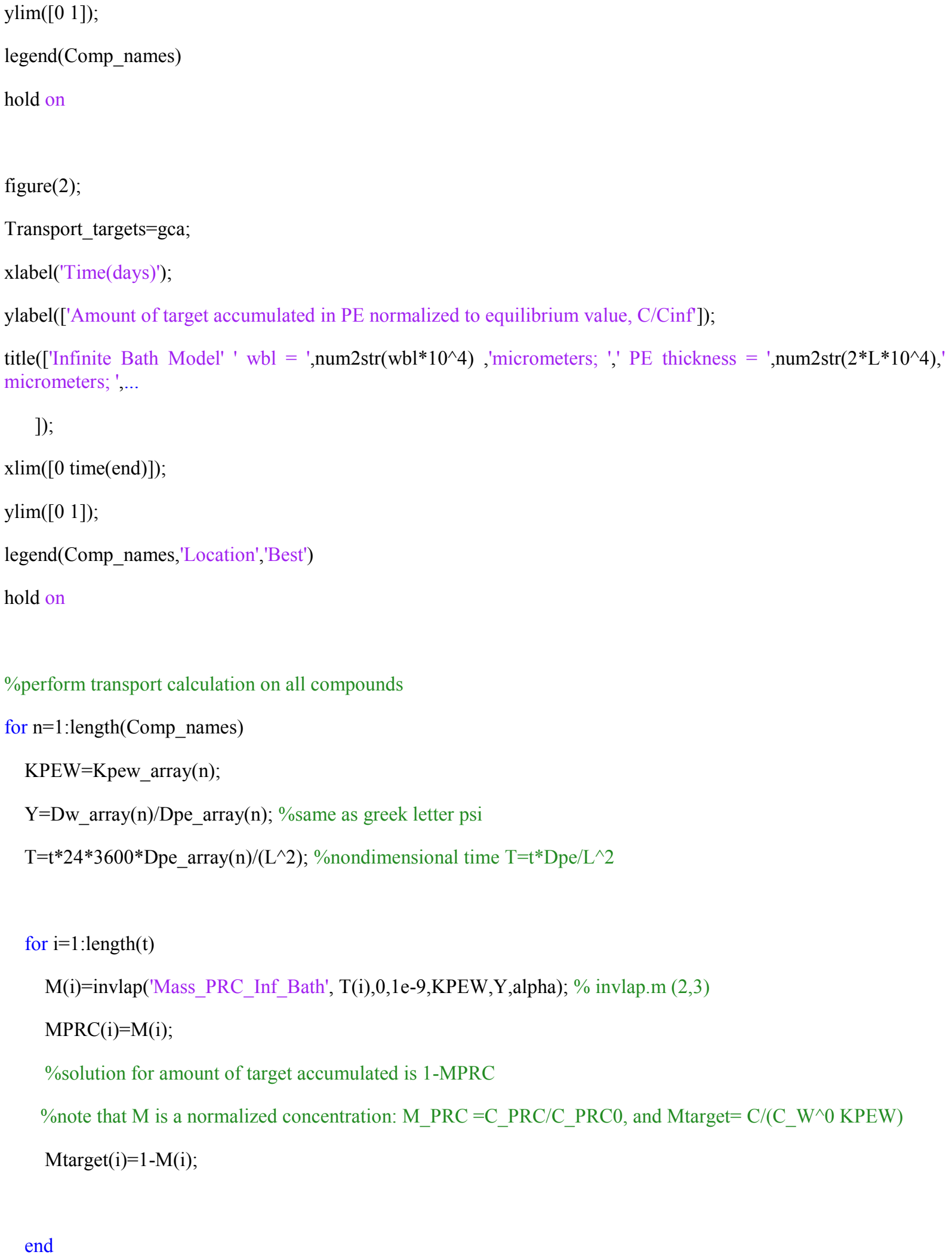


$\%$ plot result of transport calculation for compound $n$

plot(Transport_PRC,t,MPRC,linespec_array(n))

plot(Transport_targets,t,Mtarget,linespec_array(n))

end

hold off 
Table S1. Physico-chemical properties of various PCBs and DDTs used for fitting the uptake into PE data, with the finite bath model (Figure 3 in main text and Figure S4).

\begin{tabular}{|l|r|r|r|}
\hline Chemical & $\begin{array}{c}\log K_{P E W} \\
\left(L_{W} / k g_{P E}\right)^{l}\end{array}$ & $\begin{array}{l}\log D_{P E} \\
\left(\mathrm{~cm}^{2} / \mathrm{s}\right)^{2}\end{array}$ & $\begin{array}{c}\log D_{W} \\
\left(\mathrm{~cm}^{2} / \mathrm{s}\right)^{3}\end{array}$ \\
\hline PCB 8 & 4.6 & -8.64 & -5.24 \\
PCB 18 & 4.84 & -8.81 & -5.28 \\
PCB 28 & 5.26 & -8.81 & -5.28 \\
PCB 44 & 5.37 & -8.98 & -5.32 \\
PCB 52 & 5.43 & -8.98 & -5.32 \\
PCB 66 & 5.94 & -8.98 & -5.32 \\
PCB 77 & 5.79 & -8.98 & -5.32 \\
PCB 101 & 6.19 & -9.16 & -5.36 \\
PCB 105 & 6.36 & -9.16 & -5.36 \\
PCB 118 & 6.42 & -9.16 & -5.36 \\
PCB 126 & 6.6 & -9.16 & -5.36 \\
PCB 128 & 6.52 & -9.33 & -5.39 \\
PCB 138 & 6.66 & -9.33 & -5.39 \\
PCB 153 & 6.81 & -9.33 & -5.39 \\
PCB 170 & 7.05 & -9.50 & -5.41 \\
PCB 180 & 7.2 & -9.50 & -5.41 \\
PCB 187 & 7.12 & -9.50 & -5.41 \\
2,4'-DDE & 5.62 & -9.31 & -5.32 \\
4,4'-DDE & 5.77 & -9.33 & -5.32 \\
2,4'-DDD & 4.94 & -9.36 & -5.35 \\
4,4'-DDD & 4.89 & -9.39 & -5.35 \\
2,4'-DDT & 5.76 & -9.59 & -5.38 \\
4,4'-DDT & 5.59 & -9.59 & -5.38 \\
\hline
\end{tabular}

\footnotetext{
${ }^{1} K_{P E W}$ values for PCBs ${ }^{4}$, except PCB $126{ }^{5}$. For DDTs, we used the $K_{P E W}$ values from ${ }^{6}$.

${ }^{2}-\log D_{P E}\left(\mathrm{~cm}^{2} / \mathrm{s}\right)=0.0145 * \mathrm{~V}_{\mathrm{m}}+6.1^{7}$.

${ }^{3} D_{W}\left(\mathrm{~cm}^{2} / \mathrm{s}\right)=\left(2.7^{*} 10^{-4}\right) /\left(\mathrm{MW}^{0.71}\right)^{8}$.
} 


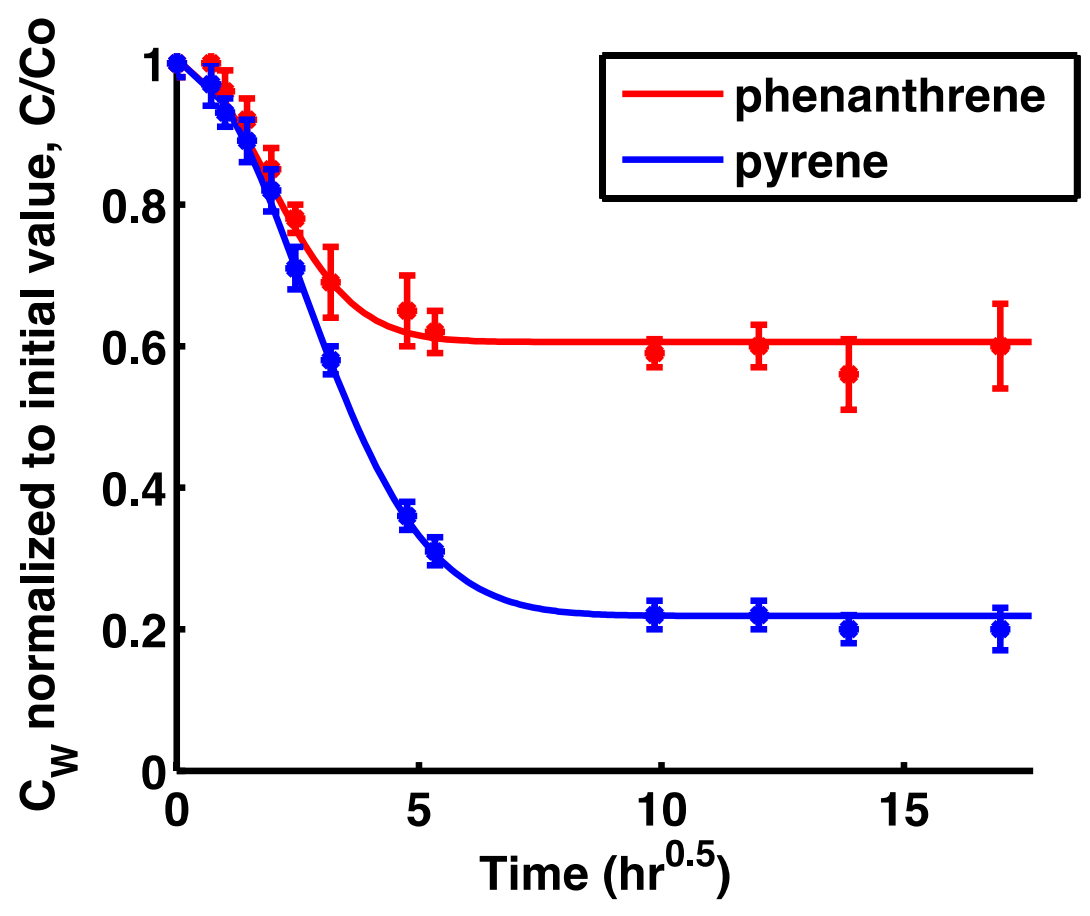

Figure S1. Model fit of the finite bath solution (main text Eq 7, inverted with Code S1) to the uptake data of phenanthrene (red) and pyrene (blue). The solid lines represent the fit with $\delta_{\mathrm{w} \text {, phen }}$ $=210 \mu \mathrm{m}$ and $\delta_{\mathrm{w}, \text { pyr }}=190 \mu \mathrm{m}$. Other model parameters used were polymer half thickness $L_{P E}$ $=12.75 \mu \mathrm{m}, m_{P E}=0.03444 \mathrm{~g}, V_{W}=0.580 \mathrm{~L}, 0.91 \mathrm{~g} / \mathrm{cm}^{3}, D_{P E, p h e n}=10^{-8.44} \mathrm{~cm}^{2} / \mathrm{s}, D_{P E, p y r}=10^{-8.76}$ $\mathrm{cm}^{2} / \mathrm{s}, K_{P E W, p h e n}=10^{4.04} \mathrm{~L} / \mathrm{kg}$ and $K_{P E W, p y r}=10^{4.78} \mathrm{~L} / \mathrm{kg}$ 

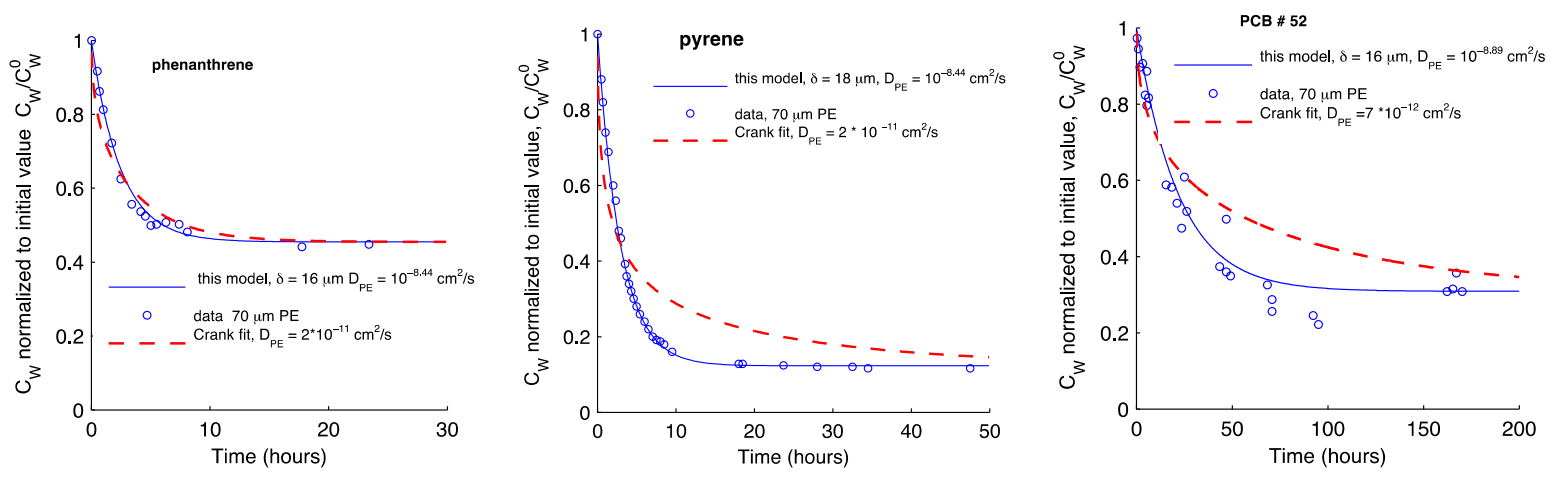

Figure S2. Finite bath model fits for sorption data of Adams et al. ${ }^{9}$ The model assuming a water boundary layer (blue lines, "this model") fits the sorption profile (blue dots) more accurately than a model assuming only diffusion into PE from a finite stirred water bath (red dashed line, "Crank fit", Equation SI 39) used by Adams et al. ${ }^{9}$.
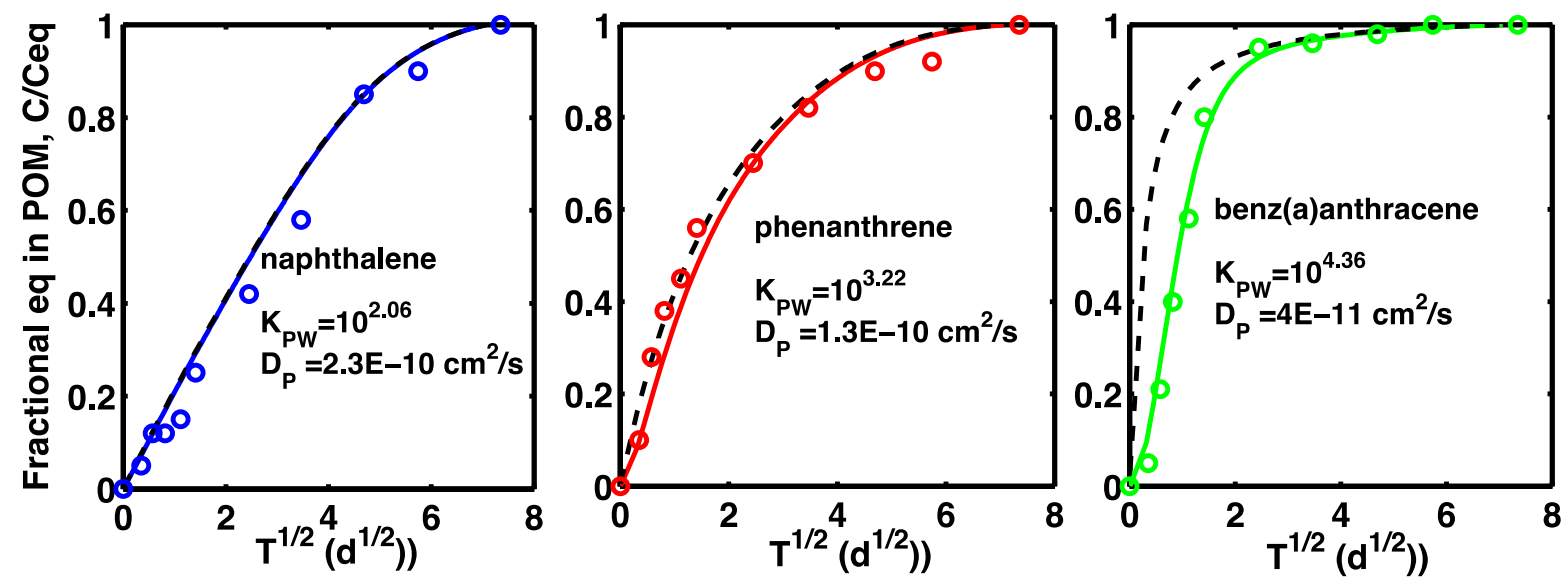

Figure S3. Application of the finite bath model (main text Eq 12, inverted with SI3) to the uptake data of naphthalene (left), phenanthrene (middle) and benz(a)anthracene(right) into POM (referred to as just $\mathrm{P}$ in the figure) from a mixed aqueous solution, obtained by Hong and Luthy ${ }^{10}$. The solid lines represent the fit with $\delta_{\mathrm{W}}=250 \mu \mathrm{m}$ and dashed black lines with $\delta_{\mathrm{W}}=0 \mu \mathrm{m}$ (essentially membrane-only solution given in Crank ${ }^{2}$ Equation SI 39). Other model parameters used were polymer half thickness $\mathrm{L}_{\mathrm{POM}}=260 \mu \mathrm{m}, \mathrm{m}_{\mathrm{POM}}=0.5 \mathrm{~g}, \mathrm{~V}_{\mathrm{W}}=0.25 \mathrm{~L}, \rho_{\mathrm{POM}}=1.38 \mathrm{~g} / \mathrm{cm}^{3}$. The presence of a boundary layer significantly improved the fit of benz(a)anthracene, for which no value of polymer diffusivity could capture the distinct uptake profile. The WBL-controlled uptake curve of benz(a)anthracene is also consistent with an exponential approach to equilibrium similar to those in Figures 1 and 4A. In contrast, the addition of an aqueous boundary layer did not change the uptake of naphthalene. Phenanthrene uptake appears to be controlled by both membrane and aqueous boundary layer, but more significantly by the membrane. 


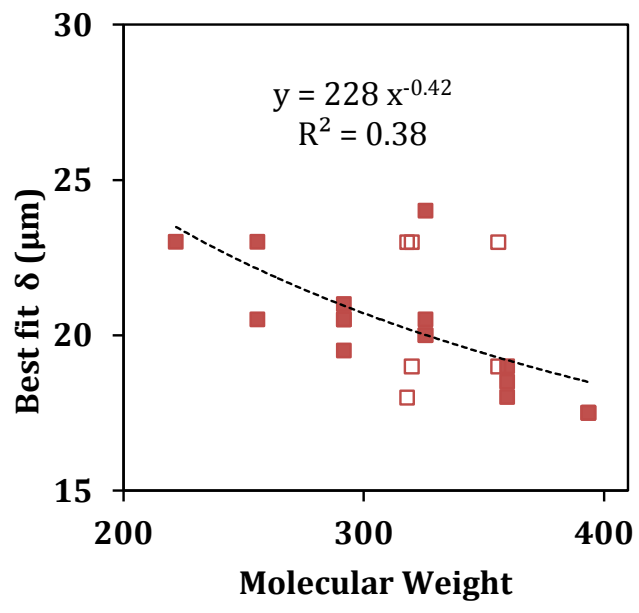

Figure S4. Best fit boundary layer thicknesses from finite bath sorption experiments versus molecular weight for 6 DDTs (no fill) and 17 PCBs (fill). The exponent of the fit is -0.42 (95\% CI $-0.66,-0.18$ ), which is consistent with empirically derived values of -0.23 from studies on transport across benthic boundary layers (see main text).

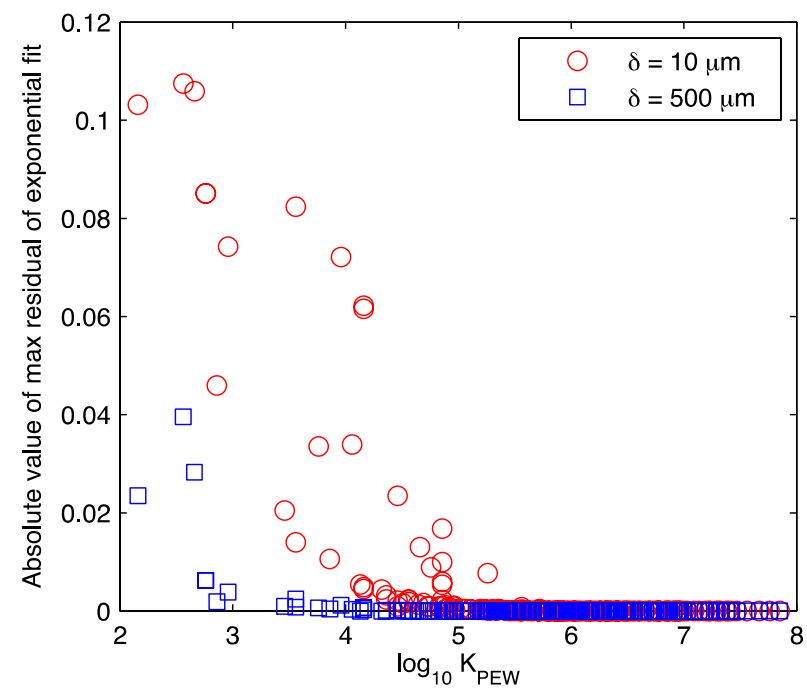

Figure $\mathrm{S} 5$. Residual of exponential fit as a function of $\mathrm{K}_{\mathrm{PEW}}$. The regressions of Equation 1 from the main text were done on uptake curves of numerous chemicals into a $100 \mu \mathrm{m}$ PE produced using numerical inversion on Eq 5 (infinite bath uptake into PE, main text) for two boundary layer thicknesses 10 (red circles) and $500 \mu \mathrm{m}$ (blue squares). Residuals of the best fit exponentials of the full solution (main text Equation 8, with Code S2) are higher for compounds with lower $K_{P E W}$ and in turbulent conditions (i.e. smaller boundary layers). 


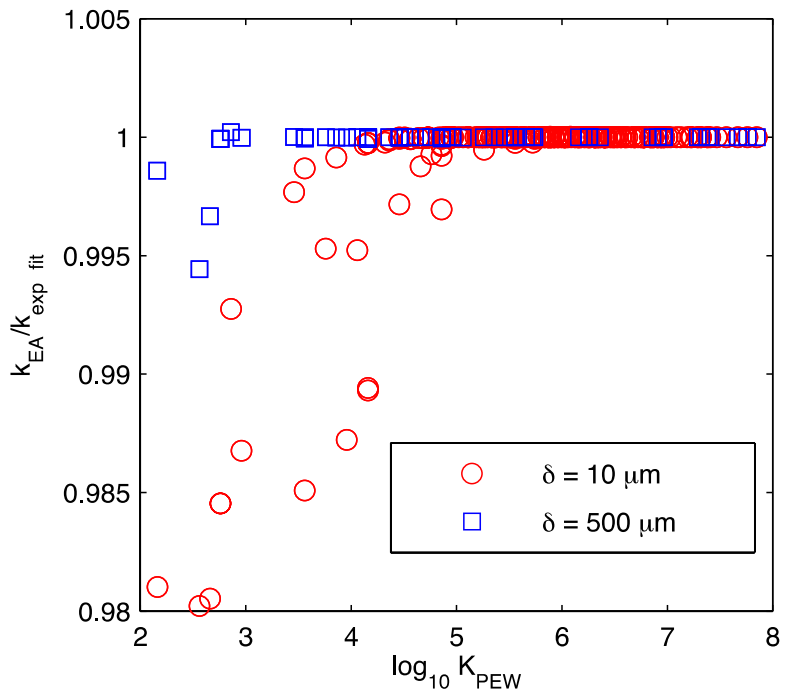

Figure S6. Ratio of exponential approximation coefficient (Equation 12 in main text) over best fit coefficient of the regression of Eq $1\left(\mathrm{k}_{\mathrm{EA}} / \mathrm{k}_{\mathrm{fit}}\right)$ to the uptake profiles of numerous chemicals as function of $\mathrm{K}_{\mathrm{PEW}}$, and assuming two boundary layer thicknesses 10 (red circles) and $500 \mu \mathrm{m}$ (blue squares). The average $\mathrm{k}_{\mathrm{EA}} / \mathrm{k}_{\text {fit }}$ ratio was $0.9993 \pm 0.0030$ for $10 \mu \mathrm{m}$ aqueous boundary layer, and $0.9998 \pm 0.0008$ for $500 \mu \mathrm{m}$. The exponential approximation (Equation 10 main text) and the sampling rate model (Equation 1) are essentially the same, when Equation 1 is fitted to the entire uptake profile.
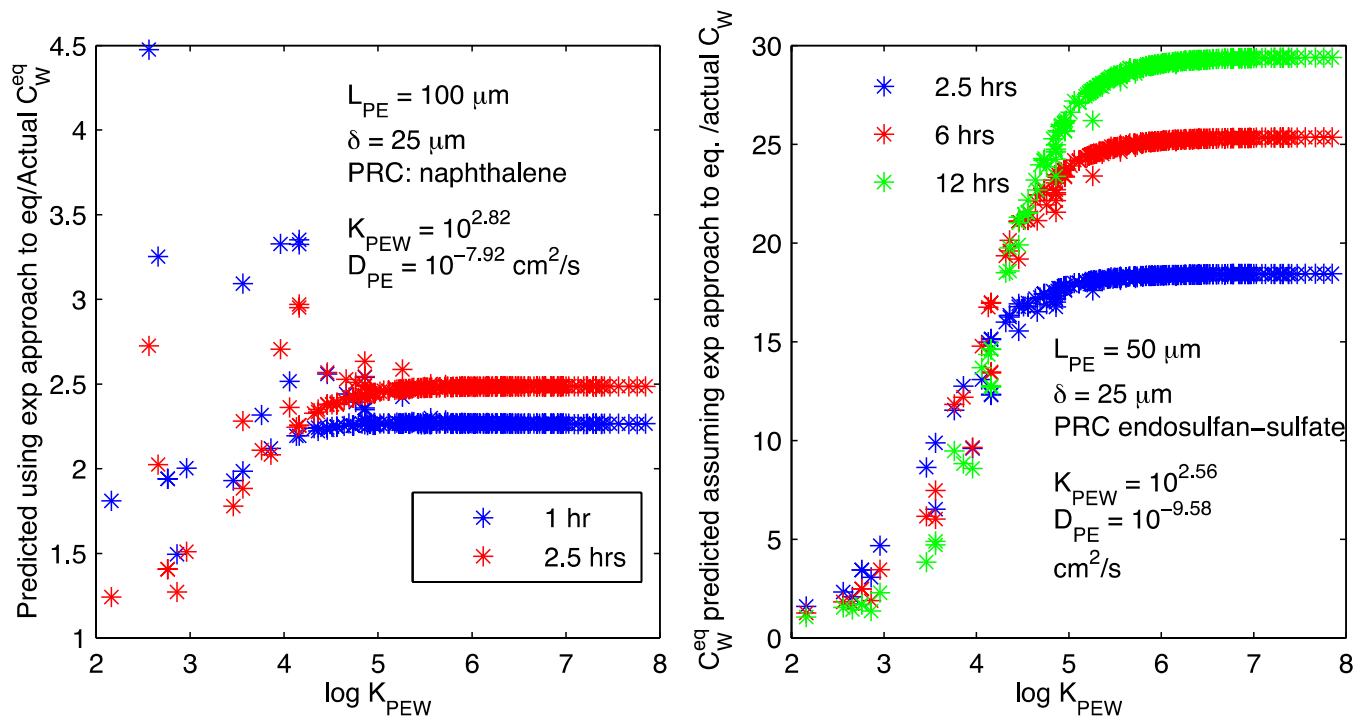

Figure S7 Ratio of predicted to actual concentrations obtained when estimating concentration at equilibrium of various HOCs by assuming exponential approach to equilibrium for PRC whose transfer is partially (naphthalene) or fully membrane (endosulfan-sulfate) controlled. Simulations were performed for various deployment times as noted in the legends. The purpose of this exercise was to investigate errors incurred when extrapolating from small, fast exchanging PRCs 
to hydrophobic chemicals, while assuming the transfer of PRCs and targets were both governed by Equation 1 of main text.
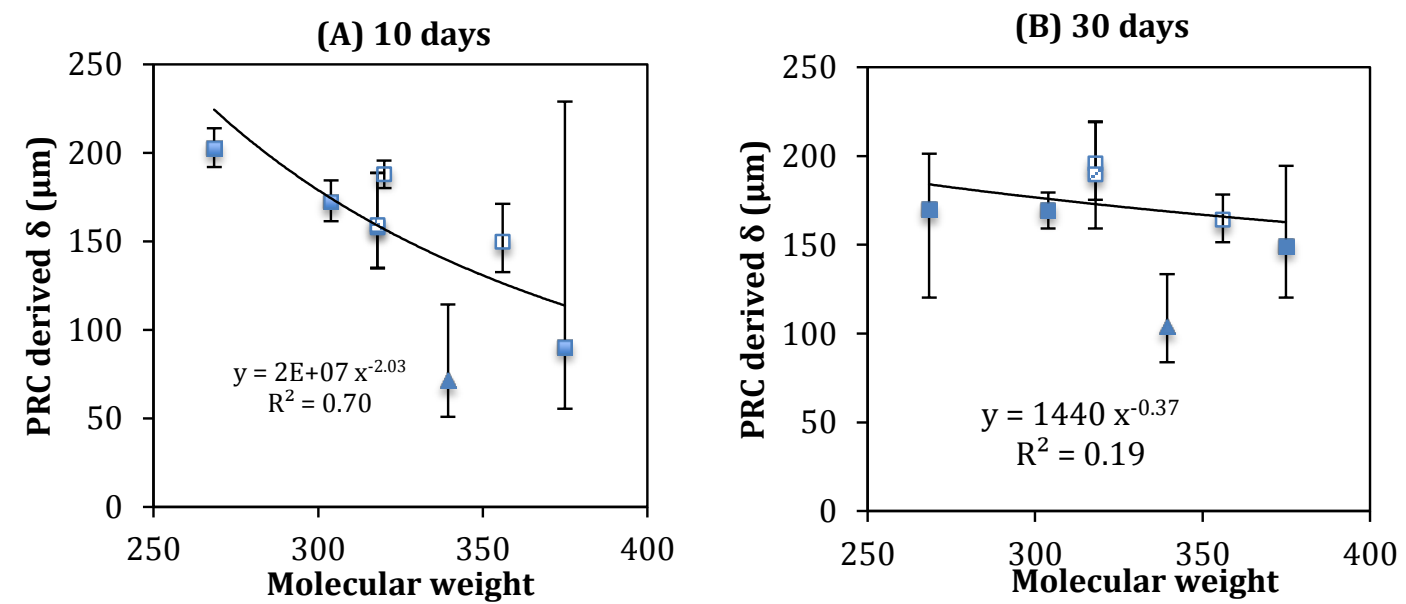

Figure S8. Boundary layer thickness based on fraction of seven PRCs (PCBs - filled, DDTs empty squares) remaining at 10 days (A) and 30 days (B) in $25 \mu \mathrm{m}$ PE and one PRC $\left({ }^{13} \mathrm{C} 4,4\right.$ ' $\mathrm{DDE}$ ) in $10 \mu \mathrm{m}$ PE (dotted square). The fitted line is based on all PRCs, except ${ }^{13} \mathrm{C}$ PCB 111 (triangle), which was considered an outlier. Error bars represent error propagation of one standard deviation in PRC concentrations in pre-deployment PE and instrumental error. For the 10 day deployment the exponent of the fit was $-2.03 \pm 0.60(p<0.05, N=7)$, but if we perform the fit without the last point $\left({ }^{13} \mathrm{C}\right.$ PCB 153 was lost only $5 \%$ ), we obtain an exponent of -1.01 , which is no longer statistically significant $(p=0.075)$.
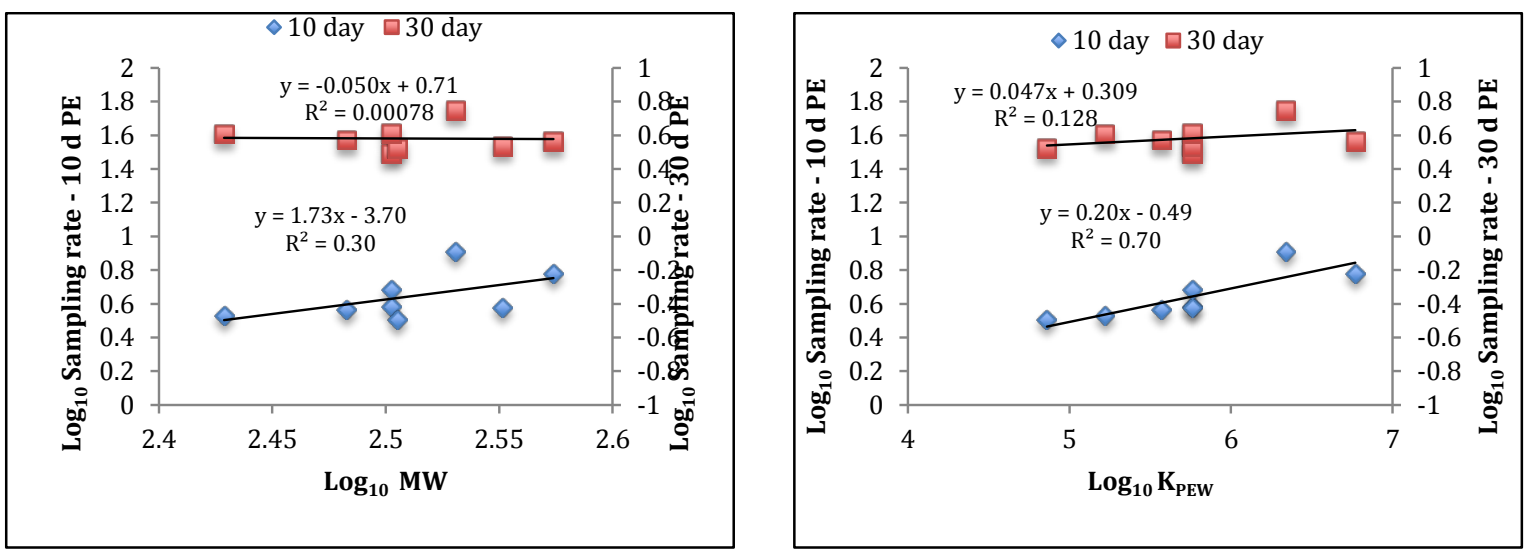

Figure S9. Sampling rate as a function of molecular weight (left) and $\mathrm{K}_{\mathrm{PEW}}$ (right) deduced from dissipation of PRCs from field deployed PE. 

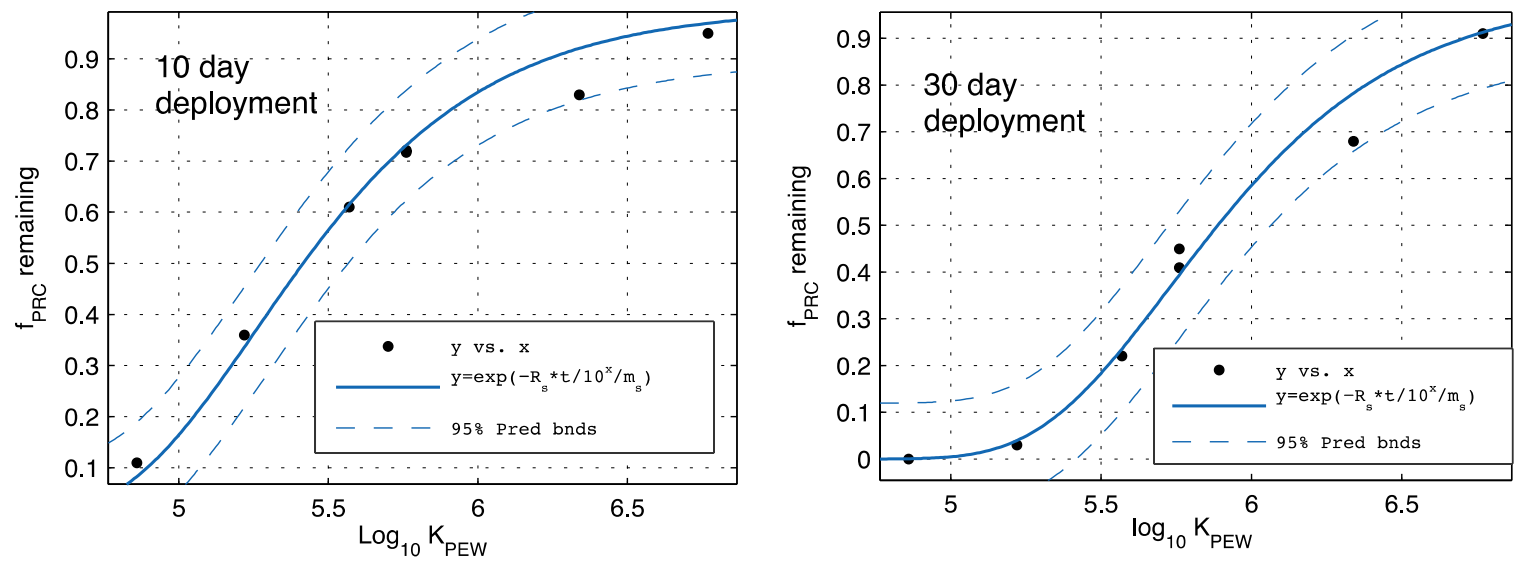

Figure S10. Example of non-linear least squares fit, following the method of Booij and Smedes ${ }^{11}$ for determining the best fit sampling rate using all the measured PRC data from 10 day (left) and 30 day (right) deployment.

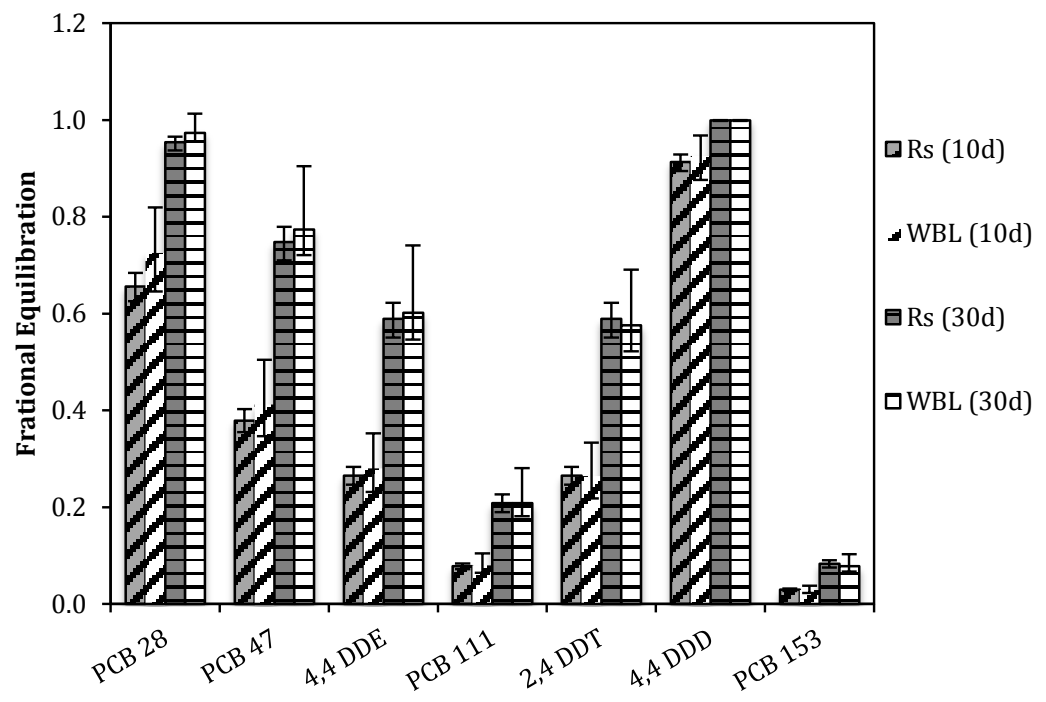

Figure S11. Fractional equilibration of various compounds calculated using sampling rate approach with NLS regression of Booij and Smedes ${ }^{11}$ (grey fill), and using the boundary layer model (white fill) for the two deployment times 10 days (slanted stripes) and 30 days (horizontal stripes). 

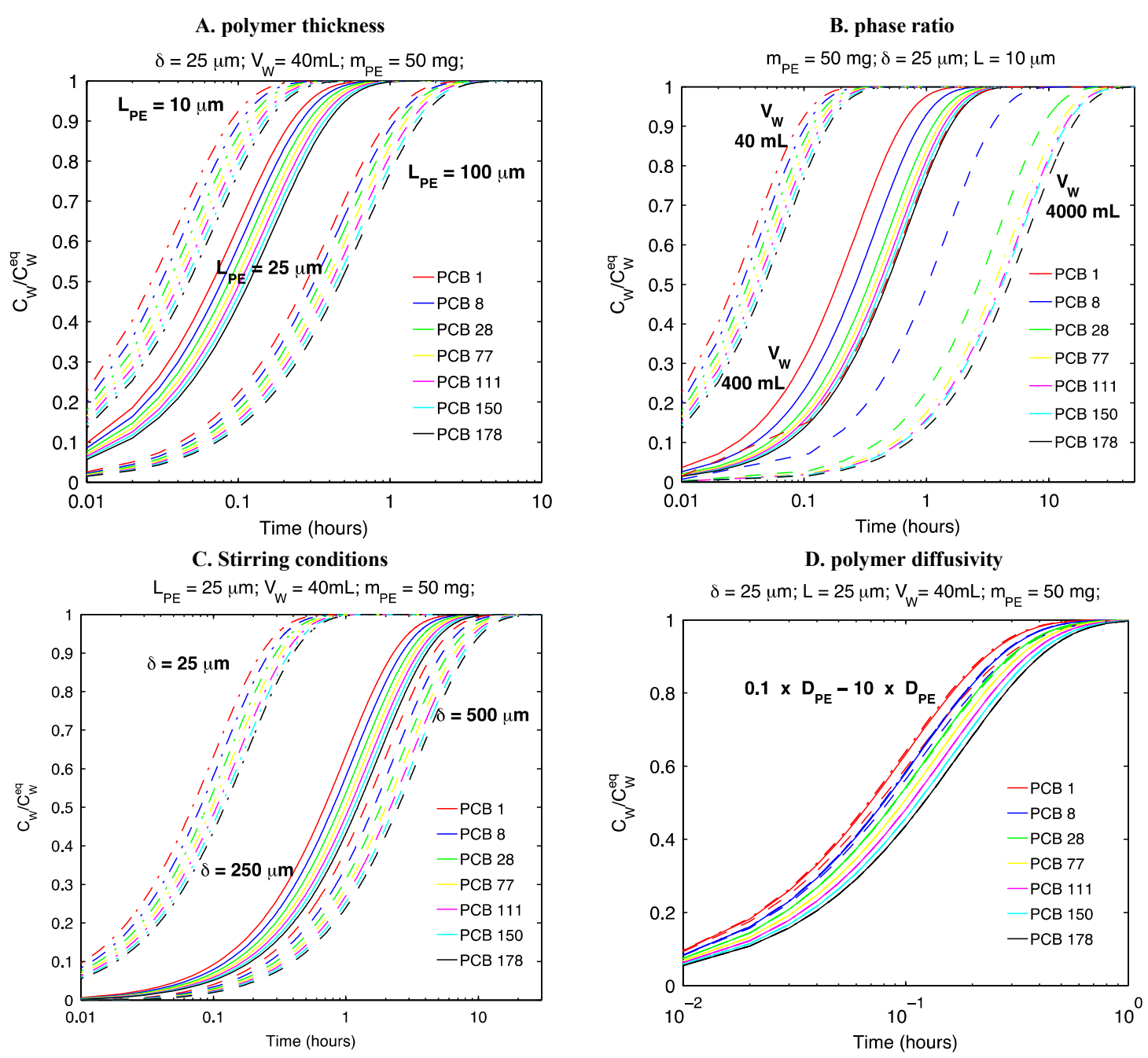

Figure S12. PE/water equilibration time for seven chemicals with $\mathrm{K}_{\mathrm{PEW}}$ ranging from 4.1 to 6.8 as a function of (A) PE thickness in a well stirred system ( $\mathrm{t}_{95 \%}$ 0.08-0.2 hours for $10 \mu \mathrm{m} \mathrm{PE}$ and 1.5-2 hours for $100 \mu \mathrm{m} \mathrm{PE})$. (B) phase ratio in a well stirred system and $10 \mu \mathrm{m} \mathrm{PE}\left(\mathrm{t}_{95 \%} 0.08-0.2\right.$ hours for $50 \mathrm{mg} \mathrm{PE} / 40 \mathrm{~mL}$ water, 2-11 hours for $50 \mathrm{mg} \mathrm{PE} / 4000 \mathrm{~mL}$ water) (C) stirring conditions for a $25 \mu \mathrm{m} \mathrm{PE}$ ( $\mathrm{t}_{95 \%} 0.1$ to 0.3 hours in well stirred conditions versus $2-4$ hours for gently stirred and 8-9 hours assuming $\delta=500 \mu \mathrm{m}$ "static" conditions). (D) diffusivity in polymer relative to diffusivity in $\mathbf{P E}$ ( $\mathrm{t}_{95 \%}$ appears to be fairly independent of the value of diffusivity in PE over 2 orders of magnitude: 0.1 - 10 times the $\mathrm{D}_{\mathrm{PE}}$ values calculated based on molar volume with the relationship from Lohmann ${ }^{7}$ ). In all four panels, the parameters needed to run the model (PE and $\delta$ thicknesses, mass of $\mathrm{PE}$ and volume of water) are displayed above each panel. 


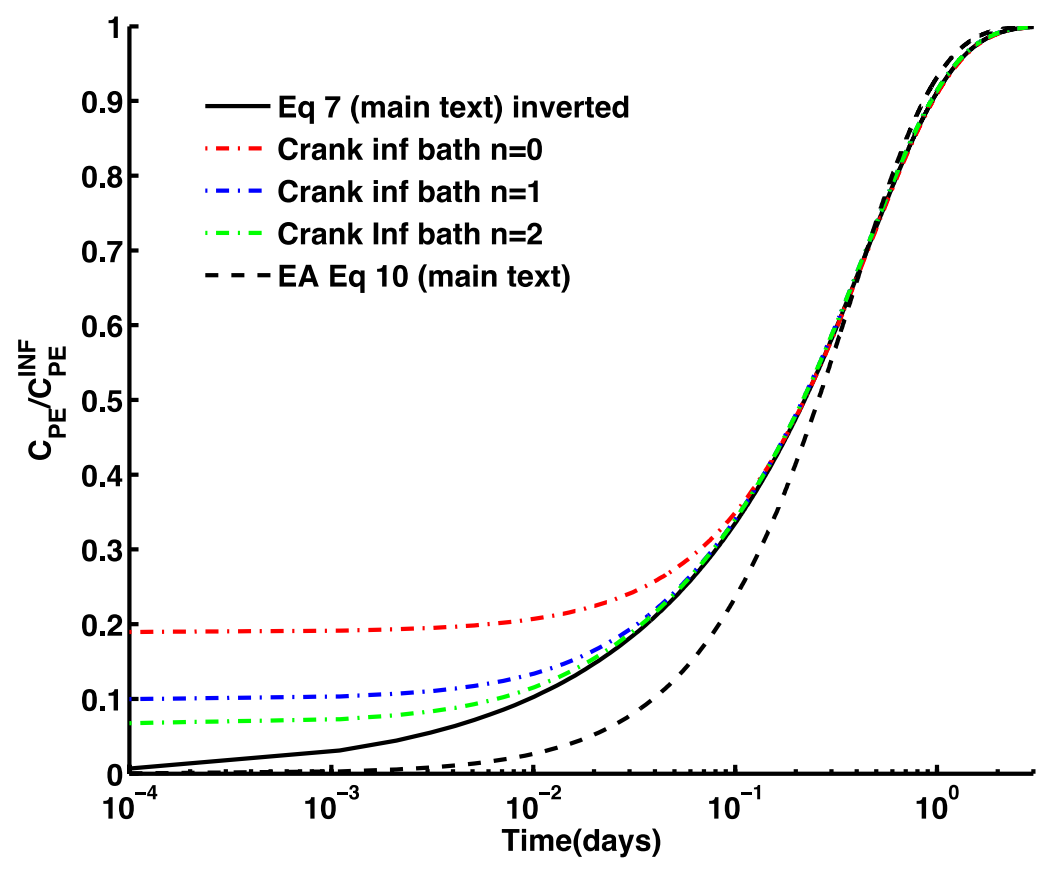

Figure S13. Comparison of various uptake models applied to endosulfan sulfate, a membrane controlled chemical. Solid black line is the full solution (Eq 8 with Code S2 inversion), the red blue green dotted-dashed lines are the Crank solutions for diffusion in a plane membrane from a solution of constant concentration and infinite volume ${ }^{2}$ (Eq 4.42, also reproduced as Eq 54 in Text S1, and using $n$, defined in Equation 54, of $n=0, n=1$ and $n=2$, respectively). For times greater than $1 \%$ of the equilibration time, the Crank solution with $n=2$ (corresponding to 3 terms in the sum of exponentials of Eq 54) gives suitable agreement with the full solution for times greater than $1 \%$ of the equilibration time, whereas $n=0$ (only one exponential term) holds for times greater than $10 \%$ of the equilibration time. Dashed line is the exponential approximation developed in the main text (Equation 10), but as stated in the text and evidenced in the figure, it is not applicable to membrane-controlled chemicals. 


\section{References}

1. Hollenbeck, K. J. INVLAP.M: A matlab function for numerical inversion of Laplace transforms by the de Hoog algorithm http://www.isva.dtu.dk/staff/karl/invlap.htm

2. $\quad$ Crank, J., The mathematics of diffusion. 2nd ed.; Oxford University Press: Oxford, 1975; p 44-68.

3. Booij, K.; Vrana, B.; Huckins, J. N., Theory, modelling and calibration of passive samplers used in water monitoring. In Passive Sampling Techniques in Environmental Monitoring, Greenwood, R.; Mills, G.; Vrana, B., Eds. Elsevier Science Bv: Amsterdam, 2007; Vol. 48, pp 141-169.

4. Choi, Y.; Cho, Y. M.; Luthy, R. G., Polyethylene-water partitioning coefficients for parent- and alkylated-polycyclic aromatic hydrocarbons and polychlorinated biphenyls. Environ Sci Technol 2013, 47, (13), 6943-50.

5. Gschwend, P. M.; MacFarlane, J. K.; Reible, D. D.; Lu, X.; Hawthorne, S. B.; Nakles, D. V.; Thompson, T., Comparison of Polymeric Samplers for Accurately Assessing PCBs in Pore Waters. Environ Toxicol Chem 2011, 30, (6), 1288-1296.

6. Hale, S. E.; Martin, T. J.; Goss, K.-U.; Arp, H. P. H.; Werner, D., Partitioning of organochlorine pesticides from water to polyethylene passive samplers. Environ Pollut 2010, $158,(7), 2511-2517$.

7. Lohmann, R., Critical review of low-density polyethylene's partitioning and diffusion coefficients for trace organic contaminants and implications for its use as a passive sampler. Environ Sci Technol 2012, 46, (2), 606-18.

8. Schwarzenbach, R. P.; Gschwend, P. M.; Imboden, D. M., Environmental Organic Chemistry. 2nd ed.; John Wiley: New York, 2003.

9. Adams, R. G.; Lohmann, R.; Fernandez, L. A.; Macfarlane, J. K.; Gschwend, P. M., Polyethylene devices: Passive samplers for measuring dissolved hydrophobic organic compounds in aquatic environments. Environ. Sci. Technol. 2007, 41, (4), 1317-1323.

10. Hong, L.; Luthy, R. G., Uptake of PAHs into polyoxymethylene and application to oilsoot (lampblack)-impacted soil samples. Chemosphere 2008, 72, (2), 272-281.

11. Booij, K.; Smedes, F., An improved method for estimating in situ sampling rates of nonpolar passive samplers. Environ Sci Technol 2010, 44, (17), 6789-94. 



\section{Chapter 5.}

\section{Characterizing baseline toxicity of HOC mixtures with GC $\times$ GC and passive dosing}

A. Patricia Tcaciuc ${ }^{1,2} *$, Robert K. Nelson ${ }^{2}$, Lauren Rotkovitz ${ }^{3}$, Christopher M. Reddy ${ }^{2}$, and Philip M. Gschwend, ${ }^{1,2}$

${ }^{1}$ Department of Civil and Environmental Engineering, Massachusetts Institute of Technology, Cambridge, MA 02139, USA

${ }^{2}$ Department of Marine Chemistry and Geochemistry, Woods Hole Oceanographic Institution, Woods Hole, MA 02543, USA

${ }^{3}$ Department of Chemical Engineering, Massachusetts Institute of Technology, Cambridge, MA 02139, USA 


\section{Abstract}

Hydrophobic organic chemicals (HOCs) are often present in contaminated sediments as complex mixtures. Although current risk assessment procedures focus on priority pollutants like PAHs or PCBs, HOCs in general are known to cause narcosis toxicity in an additive fashion by partitioning into, and disrupting the function of, membrane lipids. Thus, accurate evaluations of the hazards associated with such sediments must take into account the cumulative effects of all HOCs. In this paper, we implement a methodology that we recently developed (Chapter 3 ) for estimating the concentration of HOCs in the lipids of organisms exposed to contaminated sediments, which combines the use of polyethylene (PE) passive samplers and comprehensive two dimensional gas chromatography, coupled with flame ionization detector (GC $\times$ GC - FID). In this methodology, GC $\times$ GC - FID analysis is used to quantify the mixture components, as well as estimate their respective concentration in the lipids of an exposed organism at equilibrium with the sediment. We applied this methodology to sediments containing various amounts of PAHs, unresolved complex mixtures (UCM), as well as other HOCs, and compared the results against the results of a novel PE-based passive dosing toxicity protocol with Daphnia magna. Body burdens calculated using GC $\times$ GC-FID analysis were in good agreement with the measured baseline toxicity. Daphnia magna immobilization was observed when GC $\times$ GCestimated body burdens exceeded the baseline toxicity threshold of $0.01 \mathrm{~g} / \mathrm{g}_{\text {lipid. }}$. However, the toxicity in the passive dosing tests appeared to be driven largely by the more polar components of the HOC mixtures, such as PAHs. According to their GC $\times$ GC retention behavior, these polar constituents partitioned more favorably into lipids over polyethylene, and were thus responsible for most of the HOC body burden. However, the observed toxicity was poorly predicted when only the EPA 16 PAHs were considered, particularly in sediments with coal tar derived contamination. 


\subsection{Introduction}

Baseline toxicity, also known as narcosis toxicity, has been identified as a hazard for aquatic life. ${ }^{1,2}$ Due to their affinity for membrane lipids, various hydrophobic organic chemicals (HOCs) have been linked to this kind of toxicity. ${ }^{1,3,4}$ Narcosis toxicity is also believed to be additive, ${ }^{5,6}$ which is a concern because HOC mixtures are very common in sediments. Many industrial chemical products like fuels, lubricants and pesticides are complex mixtures made up of tens to hundreds of components (e.g., Aroclors, fuels, toxaphene). In addition, physical, chemical and biological processes can further alter the composition of such mixtures upon their release in the environment. For example, the weathering of petroleum (which can originate from natural seeps as well as anthropogenic sources) forms a mixture of recalcitrant hydrocarbons, which is also known as the unresolved complex mixture (UCM). The UCM is ubiquitous in the environment, and although its composition has not been fully elucidated, many of its components have been linked to acute or chronic toxicity. ${ }^{7-9}$

Although mixtures are common, regulatory standards and toxicity tests tend to focus on individual chemicals (i.e. priority pollutants). ${ }^{10,}{ }^{11}$ For example, polycyclic aromatic hydrocarbons (PAHs) are routinely monitored in sediments, even though PAHs can be only a small portion of the total hydrocarbons or other HOCs present at a site. ${ }^{12}$ In contrast, complex mixtures of other nonpolar pollutants have been linked to impaired mussel health ${ }^{13-15}$ and decreases in abundance of oceanic phytoplankton. ${ }^{16}$ Moreover, regulatory guidelines focus on a subset of PAHs (specifically a set of 16 PAHs which are among 127 priority pollutants identified by the US Environmental Protection Agency (US EPA)), but depending on the source of contamination, other PAH isomers and methylated derivatives can contribute to toxicity via cumulative effects. For example, in sediments collected from manufactured gas plant sites, alkylated PAHs accounted for more than $60 \%$ of the total PAHs both in concentration and observed toxicity. ${ }^{17}$ Similarly, over $90 \%$ of toxicity of diesel fuel and crude oil has been attributed to alkylated PAHs. ${ }^{17}$

Narcosis effects are additive based on toxicity units ${ }^{18}$ (ratio of concentration to LC50) or body burdens, ${ }^{6}$ but it is worth noting that chemicals for which individual dose response relationships cannot be derived, can still contribute to cumulative toxicity effects. Recent studies 
have shown that even at their solubility limits, some HOCs cannot exert acute toxicity, but they do contribute significantly to cumulative toxicity when they are part of a mixture. ${ }^{19-22}$ For PAHs with low solubility such as anthracene and chrysene, the corresponding body burdens at equilibrium with a saturated PAH solution (30 and $10 \mu \mathrm{mol} / \mathrm{g}_{\text {lipid }}$, respectively), are below the narcosis threshold $\left(40-160 \mu \mathrm{mol} / \mathrm{g}_{\text {lipid }}\right){ }^{2,}{ }^{5}$ The threshold is exceeded if both chemicals are present at solubility and as a result, the two chemicals are only toxic when they form a mixture. The toxicities of anthracene and chrysene can also be understood in terms of chemical activities (defined as the ratio of dissolved concentration to a reference concentration, taken here to be the solubility of the pure liquid or that of a hypothetical subcooled liquid for solids). Toxicity is expected to occur when the sum of chemical activities exceeds $\sim 0.01 .^{23,24}$ For compounds with melting points higher than $200{ }^{\circ} \mathrm{C}$ (and consequently, high energy costs associated with crystal dissolution), ${ }^{25}$ the maximum chemical activity they can reach at saturation (0.005 and 0.01 for anthracene and chrysene, respectively) is below or very close to the narcosis threshold. ${ }^{22}$ This implies that there may be HOCs which do not exert acute toxicity when tested individually in the laboratory, but which could contribute to cumulative toxic effects when they are part of an environmental mixture.

The toxicity of HOCs in sediments is further complicated by the presence of sorptive phases such as organic and black carbon, which partially sequester and reduce the bioavailability of HOCs. $^{26-28}$ Traditionally, bulk total petroleum hydrocarbons (TPH) measurements have been used for evaluation of petroleum contaminated sediments. ${ }^{29}$ However, TPH measurements in sediments are subject to interferences from biological carbon ${ }^{29}$ and the results can vary with the methodology used (gravimetric, gas chromatography, or spectroscopy measurements). ${ }^{29}$ In addition, TPH concentrations offer little information about the bioavailability of various mixture components; instead, because toxicity and bioaccumulation are driven by the chemical activity in sediments, the toxicity and bioaccumulation associated with petroleum-contaminated sediments were better explained by freely dissolved concentrations which were either estimated from equilibrium partition models ${ }^{30}$ or measured directly. ${ }^{31}$ For both petroleum hydrocarbons ${ }^{31}$ and other nonpolar contaminants (Greenberg et al. ${ }^{32}$ and references within), passive sampling has proven to be a useful tool for measuring freely dissolved concentrations. 
Thus, in order to address the potential for cumulative toxicity of HOC mixtures a better approach is needed which (1) is based on freely dissolved as opposed to bulk concentrations and (2) takes into account the contributions of all HOCs present. To this end, we have recently developed methods for estimating concentrations of various HOCs in the lipids of exposed organisms from corresponding concentrations in passive samplers equilibrated with sediments (Chapters 2 and 3). By making use of comprehensive two dimensional gas chromatography (GC $\times \mathrm{GC}$ ) retention times for evaluating partition coefficients between polyethylene (PE) passive sampler $\left(K_{P E W}\right)$ and water as well as between membrane lipids and water $\left(K_{P L W}\right)$, we can use an analytical method to both quantify the concentration and estimate the baseline toxicity of HOCs. Coupled with a flame ionization detector, which has a relatively constant response factor for hydrocarbons, ${ }^{33}$ this approach is particularly useful for UCMs because it enables estimation of mixture toxicity without identification of each UCM component. The baseline toxicity can then be estimated by comparing the $\mathrm{GC} \times \mathrm{GC}$ derived lipid load against the critical body burden for narcosis mentioned before $\left(8-40 \mathrm{mg} / \mathrm{g}_{\text {lipid }}\right.$, assuming an average molecular weight of 200 $\mathrm{g} / \mathrm{mol})^{2}$

The objective of this work was to compare the cumulative HOC toxicity in sediments, estimated based on $\mathrm{GC} \times \mathrm{GC}$ and passive sampling measurements, against experimental determinations of acute toxicity. The acute toxicity was measured using a novel PE-based passive dosing protocol developed for assessing toxicity of environmental HOC mixtures. In passive dosing, ${ }^{19,21,34}$ polymer films are used as the source of the test chemical in a toxicity test and in our toxicity assays, the PE equilibrated with contaminated sediment was used to establish the chemical dose. Compared to other sediment toxicity tests, ${ }^{35}$ passive dosing-mediated toxicity tests were chosen because they are more likely to reflect toxicity due to HOCs, as opposed metals and/or other inorganic porewater constituents such as ammonia. The polymer also acts to buffer losses of test chemical to the walls or the air-water interface, as well as losses due to degradation or uptake by test organisms. For example, for HOCs with $K_{O W}>10^{5}$, uptake by test organisms can significantly change the dose in a test system (e.g, at a loading of $0.5 \mathrm{~g}$ wet weight organisms $/ \mathrm{L},{ }^{36}$ aqueous concentration reduced by factor of two for $\mathrm{K}_{\mathrm{OW}}$ of $10^{5}$ and six for $\mathrm{K}_{\mathrm{OW}}$ $\sim 10^{6}$ ). Thus far, passive dosing has been applied successfully towards understanding the acute toxicity of PAH mixtures on the water flea Daphnia magna, ${ }^{19}$ the benthic amphipods, 
Orchomonella pinguis and Corophium volutator, ${ }^{22}$ as well as the brine shrimp, Artemia franciscana, ${ }^{34}$ but also for in-vitro studies. ${ }^{37}$

The first goal of this study was to implement a PE-based passive dosing protocol for evaluation of narcosis toxicity of environmental HOCs mixtures. In order to validate the PEbased passive dosing protocol, we used the protocol to characterize the dose response curve of two test chemicals, phenanthrene and 1,3,5 trichlorobenzene. Both of these chemicals were selected because they are (1) known to cause baseline toxicity and (2) their toxicity towards Daphnia magna has been previously studied. In addition, phenanthrene can be easily measured in water with fluorescence spectroscopy, which allowed us to investigate the kinetics of $\mathrm{PE} /$ water exchange. Since our ultimate goal was to use passive sampling to test the toxicity of mixtures, we tested the passive dosing protocol on mixtures of PAHs, and in particular mixtures of anthracene and benzo[ $a]$ pyrene. These two chemicals were chosen as test chemicals because in previous studies they have been identified as chemicals that are not toxic by themselves but become toxic when tested as a mixture. ${ }^{19}$ Lastly, since petroleum derived hydrocarbon mixtures often contain saturated compounds as well as aromatic ones, we used the passive dosing protocol to measure the toxicity of octadecane. Altogether, these five test chemicals were chosen because they are solids at room temperature, which means that they can be easily loaded into PE at saturation (see Section 5.2.2). In addition, phenanthrene, anthracene and benzo[a]pyrene are well known environmental contaminants (part of the 16 PAHs on the US EPA priority pollutants list).

The second goal was to compare the observed toxicity of HOC mixtures against GC $\times$ GC and passive sampler-based estimations of body burdens and toxicities For that, we applied the passive dosing protocol to test $\mathrm{PE}$ equilibrated with various contaminated sediments containing a diverse distribution of PAHs, UCM and other HOCs. In parallel, GC $\times$ GC - FID analysis of the PE equilibrated with sediment was used to estimate the cumulative body burdens of HOCs and the potential for baseline toxicity. Our working hypothesis was that the presence of other HOCs could lead to toxicities that are greater than it would be expected based only on the cumulative toxicity of the priority pollutants, which in the sediments we used were mainly PAHs. To test this hypothesis, we selected a set of sediments that contained both PAHs and UCMs in various proportions. We note that thus far, numerous studies have proposed the idea that UCM hydrocarbons present in sediments could contribute to cumulative toxicity, ${ }^{14,38}$ but very few 
studies have attempted to test these compounds at levels, and as part of mixtures, that mimic those found in the environment. ${ }^{21}$ The passive dosing procedures developed and implemented in this study offer an opportunity to study not only acute effects (as was done in this work) but also long term chronic effects of diverse sets of HOCs and their mixtures.

\subsection{Materials and Methods}

All solvents used in this study were purchased from VWR (JT Baker Ultraresi-analyzed). Store bought spring water was used for the toxicity tests and Daphnia magna culture media, while Mili-Q water was used for all other experiments. The Mili-Q water was passed through ion exchange and activated carbon system (Aries Vaponics, Rockland, MA), and exposed to UV in a TOC reduction unit (Aquafine Corporation, Valencia, CA). Neat chemicals were obtained from Sigma-Aldrich, Saint Louis, MO (phenanthrene, anthracene, 1,3,5-trichlorobenzene, benzo $[a]$ pyrene, octadecane, 1,2-dimyristoyl-sn-glycero-3-phosphocholine (DMPC) lipids), Johnson Matthey Electronics, Spokane, WA ( $n$-tetradecylbenzene), AccuStandard, New Haven, CT (1,2,3,4-tetrachloronaphthalene), Mallinckrodt (calcium chloride, sodium azide). PAH standards (EPA 16 PAHs, US 106-N) and mixtures of deuterated PAHs used as internal standards were obtained from Ultra Scientific, N. Kingstown, RI. We also used polyethylene of two different thicknesses, $100 \mu \mathrm{m}$ (4 mil Film-Gard Plastic Sheeting, Carlisle Plastics, Minneapolis, MN) and $25.4 \mu \mathrm{m}$ (1 mil Film-Gard(C) Plastic Drop Cloth, Covalence Plastics, Minneapolis, MN). The $100 \mu \mathrm{m}$ PE was equilibrated with sediment and used in toxicity tests, while the $25 \mu \mathrm{m}$ PE was used in a lipid-PE partitioning experiment.

\subsubsection{Daphnia magna culture}

Daphnia magna cultures were obtained from Carolina Biological Supply Company (Burlington, NC) and from Aquatic Research Organisms (Hampton, NH). Upon receiving, the cultures were acclimated to conditions in our laboratory $\left(20-21^{\circ} \mathrm{C}\right)$, and transferred to open glass containers (with areas at least 10 times larger than the depth to allow for oxygen exchange). The cultures flasks were not aerated and were kept in a chamber with a 12:12 light dark cycle. Neonates were transferred to different culture containers and were used for toxicity assay after 10 days. The tests were attempted with juvenile Daphnia magna, but we obtained poor survival in the controls due to the smaller individuals getting trapped between the PE and walls of the 
flasks. Every 3 d, the water was changed with fresh spring water and fresh food (dissolved yeast) was added to all culture containers. The dissolved oxygen was measured in the spring water (8.2 $\mathrm{mg} / \mathrm{L})$, culture containers $(7.7 \mathrm{mg} / \mathrm{L})$ and during the passive dosing tests $(5.6-7.7 \mathrm{mg} / \mathrm{L}$, see below).

\subsubsection{PE loading with chemicals.}

For use in kinetics experiments and passive dosing tests, $100 \mu \mathrm{m}$ PE pieces were loaded with individual chemicals at saturation from a methanol solution, according to a procedure adapted from Smith et al. ${ }^{19}$. Excess amounts of phenanthrene, anthracene and benzo[a]pyrene were placed in a $50 \mathrm{~mL}$ ground-glass stoppered flask to which methanol and PE $(\sim 5-10 \times 350 \mathrm{mg}$ pieces) were added. The loading flasks were wrapped in foil and placed on a shaker table at 100 rpm and agitated for $72 \mathrm{~h}$. Saturation was confirmed by presence of crystals at the bottom of the flask. The PE was then removed, wiped thoroughly and rinsed with Milli-Q water. To ensure desorption of any residual methanol, the wiped PE pieces were placed in another container with Milli-Q water and placed on the shaker table overnight. Finally, the PE pieces were removed and placed in water in a foil-wrapped storage flask until used.

For 1,3,5-trichlorobenzene (1,3,5-TCB) and phenanthrene, various concentrations were needed to establish a dose response curve, and this was achieved by dilutions with clean PE. Pieces of loaded and clean PE (in ratios of 1:5, 1:3, 1:1 and 3:1 for phenanthrene and 1:6.5, 1:4, 1:2 for 1,3,5-TCB) were weighed, added to $20 \mathrm{~mL}$ toxicity test vials with water, and shaken on the orbital shaker table for several days. After equilibration, which for phenanthrene, was determined by measuring the aqueous concentration according to procedures in the next section, the vials were used for toxicity assay (see 5.2.5).

\subsubsection{Characterization of PE-water equilibration timescales in static conditions.}

Five pieces of $100 \mu \mathrm{m}$ PE $(360 \pm 10 \mathrm{mg})$ loaded with phenanthrene at saturation, were each placed in a $20 \mathrm{~mL}$ vial. The PE and vials were identical to the ones used in the passive dosing protocol. A volume of $20 \mathrm{~mL}$ of water was added to each vial, and one vial was sacrificed after $20 \mathrm{~min}, 45 \mathrm{~min}, 90 \mathrm{~min}, 3 \mathrm{hr}$, and $18 \mathrm{hr}$. The aqueous concentration of phenanthrene in the sacrificed vial was measured using a 1:5 dilution and synchronous fluorescence signal at $292 \mathrm{~nm}$ (Perkin Elmer LS-50 spectrophotometer operated at scan range $270-350 \mathrm{~nm}$, at a scan speed of 
$500 \mathrm{~nm} / \mathrm{min}$, and excitation and emission slits set at $4 \mathrm{~nm}$ ). The equilibrium aqueous concentration of phenanthrene was then measured in each vial after $24 \mathrm{hrs}$.

\subsubsection{Determination of chemical concentrations in PE}

PE concentrations of phenanthrene, octadecane and 1,3,5-TCB were determined by placing 5-10 mg of loaded PE into an amber vial and adding $4 \mathrm{~mL}$ of hexane. The concentration of the extract was determined using a Carlo Erba HRGC 5300 Mega Series, equipped with $30 \mathrm{~m}$ DB-5 MS column (0.25 $\mu \mathrm{m}$ film thickness, $0.25 \mathrm{~mm}$ diameter, Agilent Technologies, Santa Clara, CA) and operated with a helium carrier gas flow of $3 \mathrm{~mL} / \mathrm{min}$. Manual injections of $1 \mu \mathrm{L}$ were performed directly on column with the oven initially set at $70{ }^{\circ} \mathrm{C}$, programmed to $275{ }^{\circ} \mathrm{C}$ at 25 ${ }^{\circ} \mathrm{C} / \mathrm{min}$, and finally held at $275{ }^{\circ} \mathrm{C}$ for $2 \mathrm{~min}$. The FID detector was set at $275{ }^{\circ} \mathrm{C}$. This temperature program was suitable for analysis of 1,3,5-TCB in under 5 minutes, and for analysis of octadecane and phenanthrene in $\sim 10$ minutes. For determination of PE concentration of benzo $[a]$ pyrene, the PE was extracted with a known volume of methanol, and the concentration of benzo $[a]$ pyrene in the extract was quantified with synchronous fluorescence at $261.9 \mathrm{~nm}$ (scan range 200-300 nm, excitation and emission slits at $5 \mathrm{~nm}$, scan speed of $500 \mathrm{~nm} / \mathrm{min}$, and a wavelength difference of $143 \mathrm{~nm}$ ). For all chemicals discussed in this section, calibration curves of at least 3 standards were analyzed with the samples, and the response remained linear across the range of interest $(5-20 \mu \mathrm{g} / \mathrm{L}$ for benzo[a]pyrene, $11-110 \mathrm{mg} / \mathrm{L}$ for 1,3,5-TCB and octadecane, 0.8-2.5 mg/L for phenanthrene).

\subsubsection{Passive dosing protocol.}

All passive dosing toxicity tests (with PE loaded with chemicals, mixtures and with PE equilibrated with contaminated sediment), were performed in clear $20 \mathrm{~mL}$ vials with $\sim 300 \mathrm{mg}$ of PE $(4.3 \times 7.9 \mathrm{~cm})$ wrapped around the walls of the vial, leading to $\sim 1 \mathrm{~cm}$ overlap. The contaminated PE was pre-equilibrated with $\sim 18 \mathrm{~mL}$ spring water in the test vials by overnight agitation on the shaker table. During this step, the vials were covered with foil to prevent exposure to light. The toxicity tests were then performed in triplicates with 10-15 adult Daphnia magna individuals per vial. PE-water and water-only controls were performed in parallel and also in triplicates, and results were discarded if the average survival in either control was below $80 \%$. After $48 \mathrm{hr}$, the Daphnia magna individuals were counted if active when the test vial was

gently shaken. For PE equilibrated with contaminated sediment, the toxicity assay was repeated 
with a $3 \mathrm{~d}$ pre-equilibration time. The dose response curves were fit with a sigmoidal concentration-response curve: ${ }^{20}$

$$
R=\frac{R_{\text {control }}}{1+10^{(\log E C 50-x) \text { Hill slope }}}
$$

where $R$ and $R_{\text {control }}$ are the responses (\% Daphnia magna immobilized) in the test and control flask, respectively, EC50 is the effective concentration (or effective chemical activity, Ea50) producing $50 \%$ of the response, $x$ is the $\log _{10}$ of concentration (or chemical activity) and the Hill slope is a fitted parameter determined by the steepness of the curve.

\subsubsection{Characterization of exposure}

Exposure during toxicity assays was described either in terms of measured aqueous concentration or chemical activity, which is given by:

$$
a=\frac{C_{i, w}}{C_{i, w}^{s a t}(L)}
$$

where $C_{i, w}$ is the aqueous concentration and $C_{i, w}{ }^{s a t}(L)$ is the concentration of the water at equilibrium with the pure liquid (or hypothetical subcooled liquid if the compound is a solid at the temperature of interest). At equilibrium, the chemical activity of a compound is equal in all phases (i.e. methanol, water, lipid or PE). Therefore, the exposure of Daphnia magna to PE loaded at saturation can be described by the maximum activity $\left(a_{\max }\right)$ that the chemical can reach in a saturated solution. The value of $a_{\max }$ can be calculated based on the compound's melting point $\left(T_{m}\right)$ and its entropy of fusion at its melting point, $\Delta_{\text {fus }} S$ :

$$
a_{\max }=e^{-\left(\frac{\Delta_{\mathrm{fus}} S}{R}\left(\frac{T_{m}}{T}-1\right)\right)}
$$

where $R$ is the gas constant $(8.31 \mathrm{~J} / \mathrm{mol} \mathrm{K})$ and $T$ is the room temperature $(298 \mathrm{~K})$. The entropy of fusion was calculated for each chemical based on its number of torsional bonds and the rotation symmetry number as described in Schwarzenbach et al. ${ }^{25}$, and the calculated $a_{\text {max }}$ values are listed in Table 5.1.

For establishing a baseline toxicity dose response curve in terms of concentrations in the lipids (which was needed for evaluating the toxicity based on GC $\times$ GC-derived lipid loads), we assumed that the Daphnia magna was in chemical equilibrium with the passive dosing PE. Then, 
the corresponding concentration in the lipid $\left(C_{\text {lipid }}\right)$ was calculated either from the measured aqueous concentration $\left(C_{W}\right)$ or the measured $\mathrm{PE}$ concentration $\left(C_{P E}\right)$ according to the following formula:

$$
C_{\text {lipid }}=C_{W} K_{K P L W}=\frac{C_{P E} K_{P L W}}{K_{P E W}}
$$

where $K_{P L W}$ and $K_{P E W}$ the phospholipid-water and PE-water partition coefficients (Table 5.1).

\subsubsection{PE equilibration with test sediments}

PE-sediment equilibrations were performed in $2 \mathrm{~L}$ or $4 \mathrm{~L}$ jars, loaded with at least $1.6 \mathrm{~kg}$ of wet sediment and $3 \times \sim 350 \mathrm{mg}$ pieces of $100 \mu \mathrm{m}$ thick PE. The jars with sediment and PE were placed on a roller table for 1 mo at room temperature, and to each sediment jar sufficient water $(\sim 500 \mathrm{~mL})$ was added to ensure that the sediment was continuously moving when rolled. Seven test sediments were employed to reflect various patterns of HOC contamination. The sediments were either freshly collected (Island End, Chelsea, MA, two sections: surface 0-7 cm, and deep 7-14 cm) or archived samples from previous research projects. As mentioned in Chapter 3, Island End was chosen because it contained historical coal tar contamination, which was distinctly visible in the deep sediment. Two sediment samples previously collected from Tabbs Bay, TX were chosen because they contained little PAH contamination but a predominant UCM signal derived from oil field produced water discharge. Additionally, two sediment samples, C1800 and 303.1, previously collected from Lauritzen Channel in Richmond Harbor, Richmond, CA were also used. Though C1800 sediment comes from a Superfund site undergoing remediation due to contamination with DDT and dieldrin (caused by on-shore pesticide packing and shipping activities until 1966), the sediment also contained a significant PAH signal along with a lateeluting UCM (see also Chapter 3). Lastly, two reference sediments were included (Maneer, a reference site for Tabbs Bay and 303.1, the reference sediment for C1800). These reference sediments were not expected to have toxicity, thus serving as a sediment control (in addition to water and PE-water controls). 


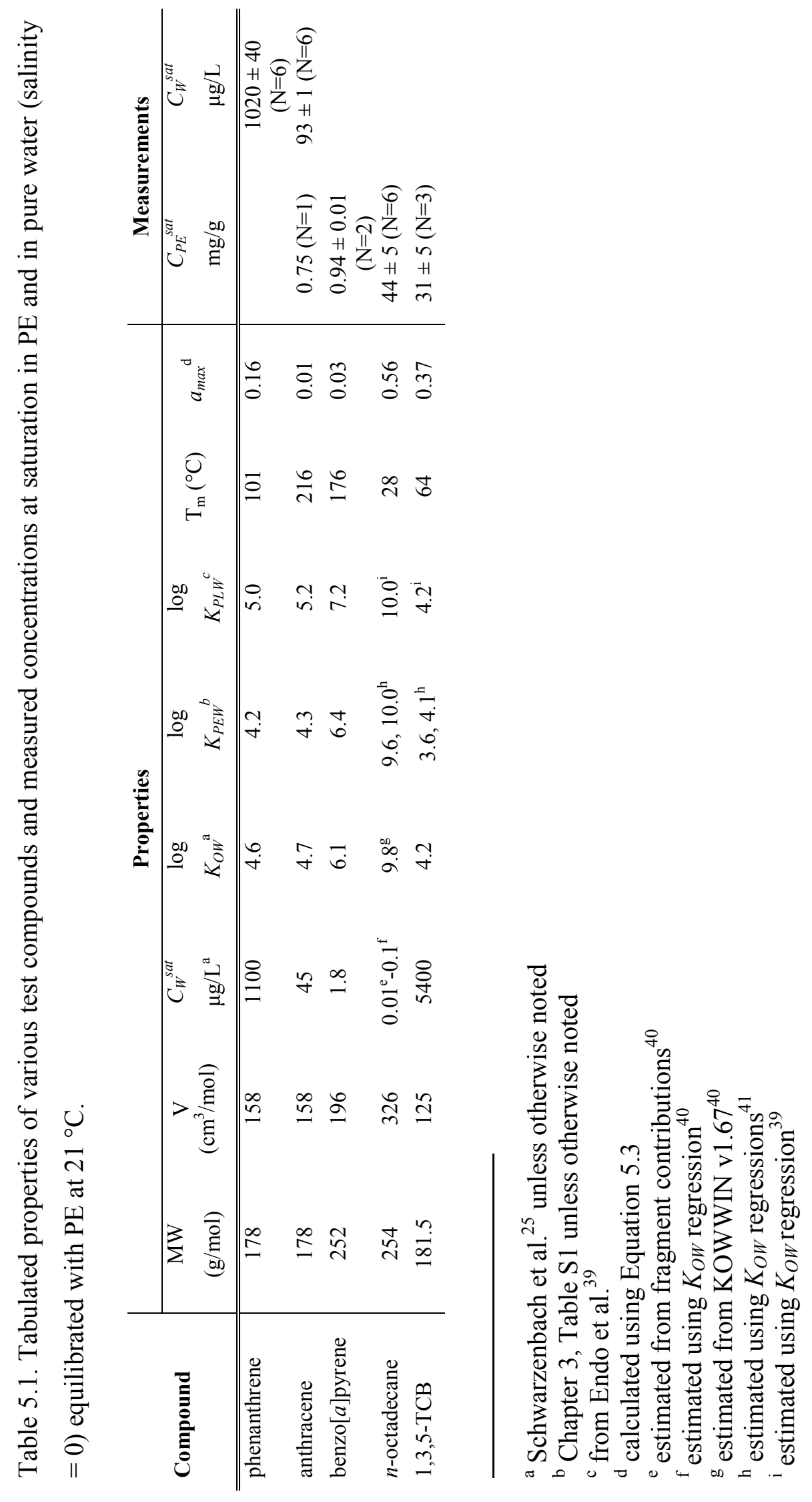




\subsubsection{PE extraction and HOC quantification}

PE equilibrated with sediment was spiked with surrogate standards ( $d_{8}$-acenapthene, $m$ terphenyl, $d_{12}$-perylene) and extracted three times with dichloromethane. A combined sample made of cuttings from each of the three $100 \mu \mathrm{m}$ PE pieces used in the toxicity assays (total of $\sim 80-100 \mathrm{mg}$ ) was extracted. The same procedure was repeated after the three pieces of PE were used in the passive dosing experiments, to confirm that the concentration of HOCs remained the same. Finally, the combined extracts were concentrated to $100-500 \mu \mathrm{L}$ and spiked with injection compounds ( $d_{10}$-anthracene, $d_{10}$-fluoranthene, $d_{12}$-benz $[a]$ anthracene) prior to analysis on the GC $\times$ GC.

\subsubsection{GC $\times$ GC-FID analysis and data processing}

Extracts of $100 \mu \mathrm{m}$ PE from before and after Daphnia magna tests were analyzed on GC $\times$ GC-FID. The analysis was performed on an Agilent 7890 GC-FID system, with a dual stage cryogenic modulator (Leco, Saint Joseph, MI). The injections were performed in splitless mode at $300{ }^{\circ} \mathrm{C}$ and the $\mathrm{H}_{2}$ carrier gas flow was maintained at $1 \mathrm{~mL} / \mathrm{min}$ throughout the run. The first dimension separation was performed on a Rxi-MS column ( $60 \mathrm{~m}, 0.25 \mathrm{~mm}$ ID and $0.25 \mu \mathrm{m}$ film thickness). The oven housing the first dimension column was held at $80^{\circ} \mathrm{C}$ for 0.2 min then the temperature was ramped to $325{ }^{\circ} \mathrm{C}$ at $2{ }^{\circ} \mathrm{C} / \mathrm{min}$ with a final hold time of $0.5 \mathrm{~min}$. The flows exiting the first dimension column were modulated at $6 \mathrm{~s}$ modulation time and the compounds were transferred onto the second dimension BPX-50 column $(1.5 \mathrm{~m}, 0.1 \mathrm{~mm}$ ID and $0.1 \mu \mathrm{m}$ film thickness). The temperature of the oven housing the second dimension column was maintained at a $5{ }^{\circ} \mathrm{C}$ offset with respect to the first dimension oven throughout the run. The modulator temperature was $15{ }^{\circ} \mathrm{C}$ higher than the temperature of the second dimension oven. The FID detector temperature was set at $330{ }^{\circ} \mathrm{C}$. To obtain more information about the identity of peaks which were not found in our standards, a subset of the samples were also analyzed on a second $\mathrm{GC} \times \mathrm{GC}$ system coupled with a time-of-flight detector $(\mathrm{GC} \times \mathrm{GC}$-TOFMS, Leco, Saint Joseph, $\mathrm{MI})$.

Additional analyses were performed on standard mixtures of HOCs (EPA 16 PAHs, $n$-alkane mix, EPA 20 PCBs, linear alkyl benzenes and alkyl cyclohexanes, listed in more detail in Chapter 2 Table 2.1 and Chapter 3 Table S1) for the purpose of determining response factors as well as retention times for training the $K_{P L W}$ and $K_{P E W}$ relationships (Chapter 3). An average 
response factor was determined based on the hydrocarbons in the standard mix (PAHs, alkyl benzenes, alkanes). The procedure for deriving the retention indices and the regression lines was adapted by Nabi et al. ${ }^{42}$ and is described in detail in Chapter 3. Briefly, the retention times are transformed into retention indices by using the relative retention of compounds with respect to the $n$-alkane series. Following that, multivariable linear regressions are used to determine the relationships describing $\log K_{P L W}$ and $\log K_{P E W}$ as a function of retention indices. The temperature program used for this study is identical to the one used in Chapter 3, and thus, the same regression equations for $K_{P L W}$ and $K_{P E W}$ will be used in this chapter (Table 3.1, Equations 3.10 and 3.11).

For evaluating the lipid load of HOCs based on GC $\times$ GC-FID analysis of PE samples, the raw PE chromatograms were imported into Matlab (Mathworks, release 2013a) and the baseline chromatogram associated with a hexane blank run was subtracted. Extract volumes and recoveries were quantified using manual integration of injection and recovery compound peaks with the ChromaTOF software (LECO Corporation, St. Joseph, MI). The recoveries were generally between 70 and $130 \%$. The contributions in the PE sample chromatograms due to column bleed were also removed, and the intensity at each point was adjusted by the response factor, the extract volume and the mass of PE extracted. The PE chromatograms were not recovery corrected. Based on the values of $K_{P L W}$ and $K_{P E W}$ estimated at each point in the chromatogram (as described above and in Chapter 3), the corresponding concentration of an HOCs in the lipid was calculated the concentration at each point in the PE chromatogram:

$$
C_{i, l i p i d}=\frac{C_{i, P E} K_{i, P L W}}{K_{i, P E W}}
$$

The total HOC load in the PE and estimated in membrane lipids was then determined by integration across the $\mathrm{GC} \times \mathrm{GC}$ space of the $\mathrm{PE}$ chromatogram and the calculated PL chromatogram. The amounts of recovery and injection compounds added to the extracts were usually small compared to the loading of the PE $(<0.5 \%$ of the total load for PE equilibrated with contaminated sediments and $\sim 10 \%$ for PE equilibrated with the reference sediment and PE blanks). In addition to the total lipid load, the sum of 16 priority PAHs was determined based on integrated peaks in the LECO software. 


\subsubsection{PE/membrane vesicle partitioning}

The partitioning of chemicals between PE and DMPC vesicles was measured for four chemicals (phenanthrene, octadecane, 1,3,5-trichlorobenzene and $n \mathrm{C}_{14}$ benzene), chosen to have similar first dimension but different second dimension retention times (i.e. different polarity). To measure the partitioning, a $25 \mu \mathrm{m}$ PE strip was loaded with the four chemicals out of a 80:20 methanol:water solution. After one week, the PE was wiped and transferred to a vial with water until used. In parallel, the membrane vesicles were made using a method adapted from Vaes et al. ${ }^{43}$. In brief, $50 \mathrm{mg}$ of DMPC crystals were dissolved in chloroform. The solvent was evaporated to dryness via rotary evaporation for 3 hours, at a water bath temperature of $50{ }^{\circ} \mathrm{C}$ and a vacuum pressure of $30 \mathrm{inHg}$. The film of lipids was hydrated with $100 \mathrm{~mL}$ of $0.01 \mathrm{M}$ $\mathrm{CaCl}_{2}$ and $50 \mathrm{mg} / \mathrm{L} \mathrm{NaN}_{3}$ buffer. The resulting solution was sonicated for $1 \mathrm{hr}$ at $40{ }^{\circ} \mathrm{C}\left(>10{ }^{\circ} \mathrm{C}\right.$ above the glass transition temperature of DMPC of $24{ }^{\circ} \mathrm{C}$ ), and allowed to come to room temperature $\left(21^{\circ} \mathrm{C}\right)$. At the same time, combusted and foil lined $20 \mathrm{~mL}$ vials were prepared with 1,5 and $10 \mathrm{mg}$ pieces cut from the piece of loaded PE. Volumes of $20 \mathrm{~mL}$ of vesicle solution at varying concentrations were then added to each vial $(0.5 \mathrm{mg} / \mathrm{mL}, 0.25 \mathrm{mg} / \mathrm{mL}$ and $0.05 \mathrm{mg} / \mathrm{mL}$ corresponding to each PE size). The concentrations of lipid vesicles were chosen such that the PE:lipid ratio would be 1:10, 1:1 and 10:1, and each experiment was done in triplicate. Finally, the vials were capped with foil line caps, covered with foil to prevent exposure to light, and placed on the shaker table for 10 days. At the end of the experiment, the PE was removed, wiped with Kim-wipe and extracted with $4 \mathrm{~mL}$ of hexane. Three pieces of the original loaded PE were also extracted with hexane for characterization of the concentrations in PE before equilibration with lipid vesicles (i.e. $t=0$ ). After spiking with a known amount of 1,2,3,4tetrachloronaphthalene (TCN), an aliquot of each extract was transferred to a GC vial for analysis on a Clarus 500 GC-MS (Perkin Elmer, Waltham, MA), equipped with a DB-XLB column (30 m, $0.25 \mathrm{~mm}$ ID, $1 \mu \mathrm{m}$ film thickness, Agilent Technologies, Lexington, MA). The helium carrier gas was set at $2 \mathrm{~mL} / \mathrm{min}$ for the entire duration of the run. We used $1 \mu \mathrm{L}$ splitless injections and an injection port temperature of $260^{\circ} \mathrm{C}$. The split valve (20:1 ratio) was open after $1.5 \mathrm{~min}$. The column temperature was programmed at $80{ }^{\circ} \mathrm{C}$ for $1 \mathrm{~min}$, then increased at 25 ${ }^{\circ} \mathrm{C} / \mathrm{min}$ to $250{ }^{\circ} \mathrm{C}$, where it was maintained for $6 \mathrm{~min}$. The lipid-PE partitioning was evaluated based on the initial and final concentration of chemicals in PE from set of lipid-PE phase ratios 
(i.e. $1: 10,1: 1$ or $10: 1)$ in for which loss of chemical from PE to the membrane vesicles was measured to be between 20 and $80 \%$.

\subsection{Results}

\subsubsection{PE loading with chemicals at saturation}

The loading of chemicals into PE at saturation was fairly uniform (variability of 10-12\% based on 3-6 replicates), and the measured concentrations in PE were consistent with saturation levels estimated from literature data (Table 5.1). The concentration in PE was largest for the aliphatic octadecane $(44 \pm 5 \mathrm{mg} / \mathrm{g})$, but smaller for the more polar benzo[ $a]$ pyrene $(0.94 \pm 0.01$ $\mathrm{mg} / \mathrm{g})$ and anthracene $(0.75 \mathrm{mg} / \mathrm{g})$. The measured PE concentrations compared favorably with estimates based on tabulated values for aqueous solubility and $K_{P E W}\left(C_{P E}^{\text {sat }}=K_{P E W} C_{W}^{\text {sat }}\right.$, Table 5.1). For example, the measured and estimated $C_{P E}^{\text {sat }}$ values were within $20 \%$ for anthracene, within a factor of 2 for $1,3,5-\mathrm{TCB}$ and a factor of 5 for benzo[ $a]$ pyrene. These factors are acceptable given typical uncertainty limits of 0.3-0.5 log units associated with solubility and partition coefficients in the literature. In addition, the aqueous concentrations of phenanthrene and anthracene measured after equilibration with loaded PE $(1020 \pm 30$ and $93 \pm 1 \mu \mathrm{g} / \mathrm{L}$, respectively) were in good agreement ( $10 \%$ for phenanthrene and factor of 2 for anthracene) with known solubility values $^{25}$ (Table 5.1). Altogether, these results confirmed that the PE used for passive dosing experiments was indeed loaded with the chemicals at their respective saturation level for phenanthrene, 1,3,5-TCB, anthracene and benzo[a]pyrene.

\subsubsection{Kinetics of $\mathrm{PE} /$ water equilibration for phenanthrene}

The kinetics of PE/water equilibration were measured for phenanthrene in a test system similar to the one used in the passive dosing setup (20 mL vials, $\sim 300-350 \mathrm{mg}$ of $100 \mu \mathrm{m} \mathrm{PE}$ ). In the absence of mixing, phenanthrene reached PE-water equilibrium within $2 \mathrm{~h}$ (Figure 5.1). The PE-water exchange model in Chapter 4 fit the release profile of phenanthrene very well, suggesting that the transport was controlled by the water boundary layer (WBL) and rendering a best-fit WBL thickness of $1000 \mu \mathrm{m}$. The equilibration timescale observed in our setup was consistent with kinetics measured in other passive dosing systems, where equilibration times of $20 \mathrm{~min}^{44}$ to a few $\mathrm{h}^{22}$ were measured for PAHs desorbing from polydimethylsiloxane (PDMS) films casts on glass cuvettes and vials, respectively. 


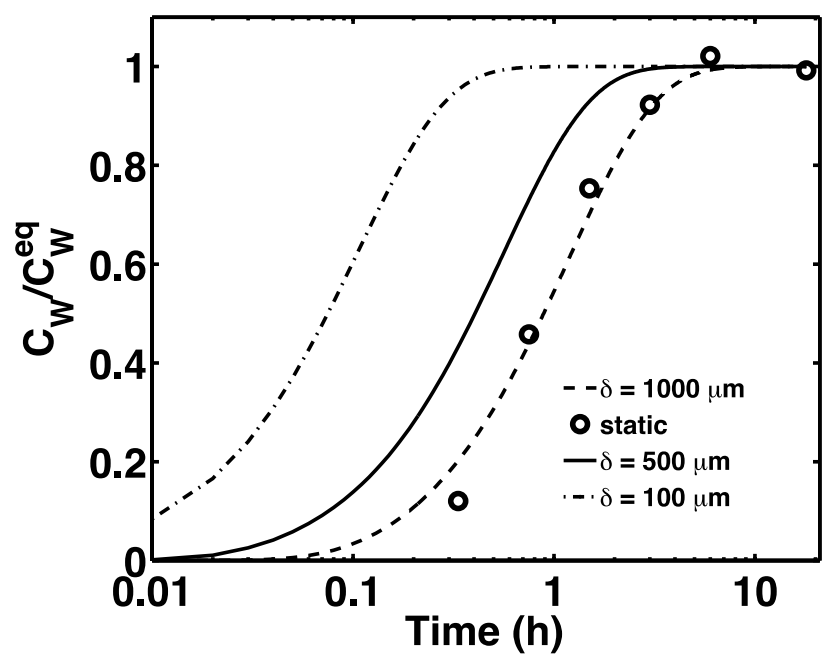

Figure 5.1 Approach to equilibrium of phenanthrene desorbing from a $100 \mu \mathrm{m}$ PE (m $\mathrm{m}_{\mathrm{PE}} 350 \mathrm{mg}$, $\mathrm{V}_{\mathrm{W}}=20 \mathrm{~mL}$ ) in static conditions. Also shown are Chapter 4 model results with aqueous boundary layer thicknesses of $1000 \mu \mathrm{m}$ (best fit for static conditions), and $500 \mu \mathrm{m}$ and $100 \mu \mathrm{m}$ (increasing degrees of mixing).

The fast equilibration timescale of $2 \mathrm{~h}$ for phenanthrene in static conditions implies that the PE-water exchange is sufficiently fast to buffer changes in chemical activity, particularly on the $48 \mathrm{~h}$ timescale of the toxicity assays. Additionally, since phenanthrene reached equilibrium in $2 \mathrm{~h}$ in static conditions, this also implies that a 1 day pre-equilibration on the shaker table before the addition of Daphnia magna, should allow sufficient time for complete PE-water equilibration. In both of these cases (during the pre-equilibration and in the presence of Daphnia magna), the equilibration timescales are expected to be shorter than in static conditions, because the aqueous boundary layers are expected to be smaller (equilibration times reduced to $0.2 \mathrm{~h}$ for WBL of 100 $\mu \mathrm{m}$, Figure 5.1).

\subsubsection{Baseline toxicity of test chemicals}

The PE-mediated passive dosing protocol and Daphnia magna toxicity assay were successfully used to measure the dose response curves for phenanthrene and 1,3,5-TCB. The calculated EC50 for phenanthrene (from fitting the dose response Equation 5.1) was $490 \mu \mathrm{g} / \mathrm{L}$ (95\% C.I. 440 to 530), which matched very well the EC50 measured by Smith et al. ${ }^{22}$ (480 $\mu \mathrm{g} / \mathrm{L}$ with $95 \%$ CI of 450 to $505 \mu \mathrm{g} / \mathrm{L}$ ). The exposure concentration was confirmed by measuring the aqueous concentration of phenanthrene at equilibrium with the PE before and after it was used in the Daphnia magna experiment (the PE was wiped and rinsed after removal from toxicity test 
vial). In general the differences in aqueous concentrations before and after use of PE in the toxicity assays were small (up to $15 \%$ ), confirming the ability of the PE to maintain constant chemical activities. It is interesting to note that while phenanthrene and anthracene are similar in terms of size and hydrophobicity, they are different in terms of toxicity. At their respective solubilities, phenanthrene caused complete Daphnia magna immobilization whereas anthrancene showed no toxicity. As mentioned previously, the lower toxicity of anthracene can be explained by its lower solubility which at saturation leads to a (1) a lower chemical activity (Table 5.1) and (2) a lower corresponding concentration in the membrane lipids than phenanthrene.

The chemical activity corresponding to $50 \%$ immobilization (Ea50) for 1,3,5-TCB was measured to be 0.069 ( $95 \%$ C.I. 0.057 to 0.084 ). The aqueous concentration of $1,3,5$-TCB was not quantified, but the variability in the PE concentration before and after use in toxicity test was less than the loading variability (10\%), suggesting that the 1,3,5-TCB concentration in PE did not change during the experiment. Previously measured EC50 values of trichlorobenzene isomers in Daphnia magna immobilization tests were in the range 0.3-3 mg/L for 1,2,3-TCB and $1.2 \mathrm{mg} / \mathrm{L}$ for $1,24-\mathrm{TCB}^{45}$ which is consistent with our estimated EC50 of $1.1 \pm 0.3\left(\mathrm{EC} 50=C_{W}{ }^{\text {sat }}\right.$ $\times$ Ea50 / $a_{\max }$, Table 5.1). The Ea50 value measured for 1,3,5-TCB was also very similar to the Ea50 of phenanthrene $(0.076,95 \%$ C.I. 0.070 to 0.083 , Figure 5.2), suggesting that both chemicals have a narcotic mode of action. Based on the measured dose response curves of phenanthrene and 1,3,5-TCB (Figure 5.2), the chemical activity threshold for toxicity (defined as $10 \%$ immobilization) is 0.04 , which is consistent with previously found narcosis threshold of $0.01 .^{23,24}$ 

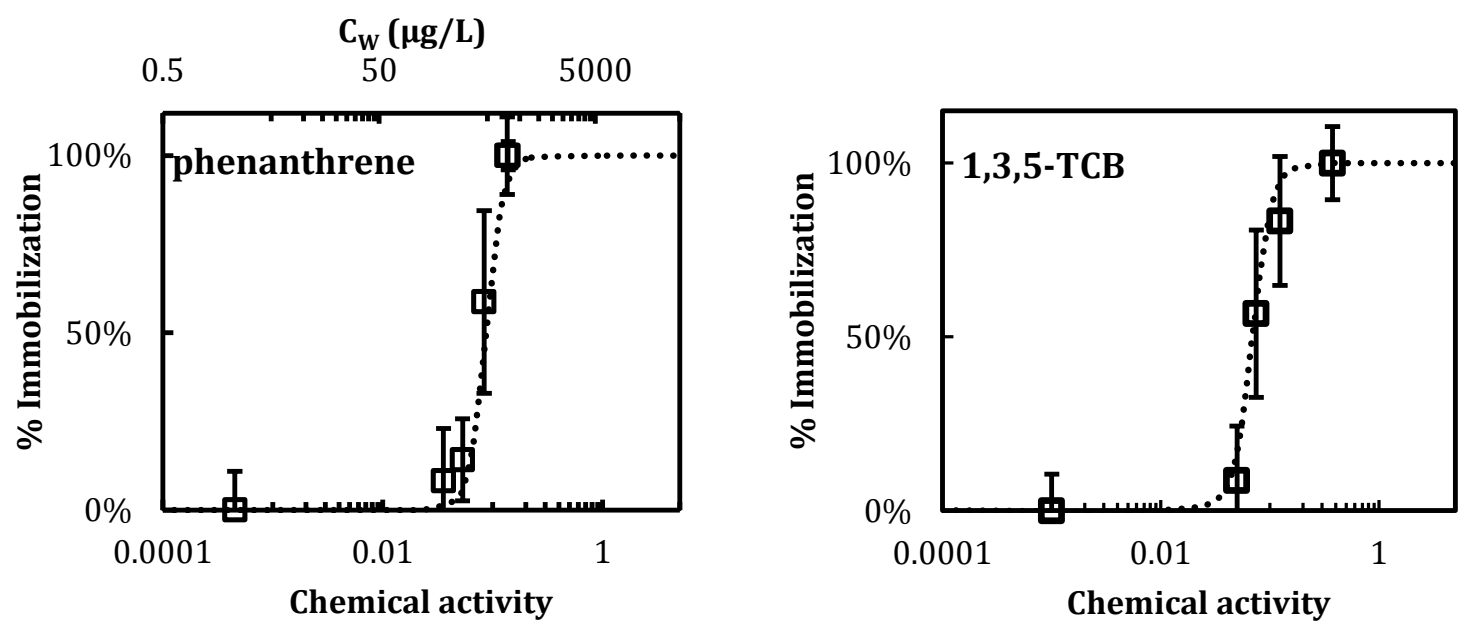

Figure 5.2 Dose response curves for phenanthrene and 1,3,5-TCB in 48 h Daphnia magna immobilization tests performed using passive dosing. Error bars represent one S.D. of triplicate measurements.

We also checked that the passive dosing protocol captured the cumulative effects of mixtures of PAHs. When present in the PE as individual chemicals at saturation, benzo[ $a]$ pyrene and anthracene showed little to no toxicity towards Daphnia magna (\% immobilization of 12 and $0 \%$, respectively). This is understandable since the chemical activity of anthracene at saturation is only 0.01 , while that of benzo $[a]$ pyrene is 0.03 (Table 5.1). In contrast, when tested as a mixture, the toxicity was significantly more (immobilization of $42 \%$ ), due to the cumulative activity of 0.04 of the two chemicals. Altogether, these results confirmed that the PE-mediated passive dosing protocol was effective at capturing the acute toxicity of hydrophobic chemicals towards Daphnia magna.

In order to use the $\mathrm{GC} \times \mathrm{GC}$-based estimations of total $\mathrm{HOC}$ concentrations to estimate toxicity, it is more useful to express the narcosis threshold in terms of lipid body burdens. Since narcosis effects are nonspecific, we combine the data obtained in this study with the PAH toxicity data (also using $48 \mathrm{~h}$ Daphnia magna immobilization endpoints) of Smith et al. ${ }^{19}$ After transforming chemical activities into lipid concentrations, and fitting a dose response curve $\left(R^{2}=\right.$ 0.84 , Figure 5.3), we derive an $\mathrm{EC}_{\text {lipid }} 50$ of $35 \mathrm{mg} / \mathrm{g}_{\text {lipid }}\left(95 \%\right.$ C.I. of 30 to $41 \mathrm{mg} / \mathrm{g}_{\text {lipid }}$ ). Fitting only the toxicity data in this study would have lead to a similarly good fit $\left(R^{2}=0.84\right)$, but slightly larger $\mathrm{EC}_{\text {lipid }} 50$ of $40 \mathrm{mg} / \mathrm{g}_{\text {lipid }}\left(95 \%\right.$ C.I. of 32 to $\left.49 \mathrm{mg} / \mathrm{g}_{\text {lipid }}\right)$. Overall the two fits lead to very similar critical body burdens for toxicity, suggesting that the results from our PEmediated passive dosing protocol are (1) in agreement with other published PDMS-based passive 
dosing protocols and (2) measuring acute toxicity consistent with narcosis toxicity. Based on the dose response curve derived in Figure 5.3, the baseline toxicity threshold (corresponding to 10\% immobilization) is $20 \mathrm{mg} / \mathrm{g}_{\text {lipid }}$ (range of 8 to $28 \mathrm{mg} / \mathrm{g}_{\text {lipid }}$ based on the $95 \% \mathrm{CI}$ values of the fitted coefficients (Table 5.1).

Lastly, since our ultimate goal was to use the passive dosing protocol to investigate the toxicity of environmental HOC mixtures, which would include besides PAHs, other hydrocarbons (HCs) with lower polarity than PAHs (e.g., monoaromatic hydrocarbons, polycyclic aliphatic compounds ${ }^{46}$ or alkanes), we also tested the baseline toxicity of octadecane. Interestingly, octadecane at saturation showed only weak toxicity $(12 \%)$, even though its maximum activity at saturation was calculated to be 0.56 (Table 5.1), a value well above the baseline toxicity threshold. Due to uncertainties in this compounds solubility and partition coefficients ( $K_{O W}$ and consequently $K_{P L W}$ and $K_{P E W}$ ), it could not be confirmed that the loading at saturation $\left(44 \pm 5 \mathrm{mg} / \mathrm{g}_{\mathrm{PE}}\right.$ ) was indeed reflective of chemical saturation for this chemical. Suspecting also that the kinetics of octadecane would be slower than that of the other chemicals tested thus far, the passive dosing was repeated with pre-equilibration times as large as 2 weeks, without any difference in the observed toxicity (immobilization of $20 \pm 8 \%$ ). The behavior of octadecane will be revisited in following sections.

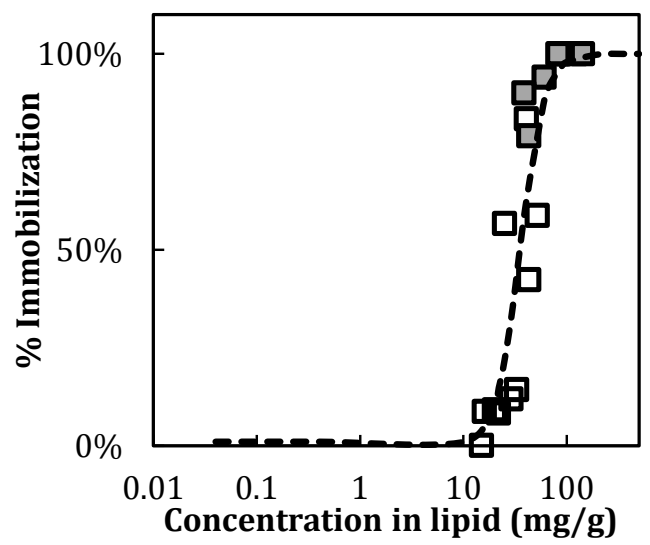

Figure 5.3 Daphnia magna immobilization plotted against concentration in the lipid (right) for chemicals test in this study (empty squares) and PAH mixtures from Smith et al. ${ }^{19}$ (filled squares). 


\subsubsection{GC $\times$ GC analysis of sediment PE}

The PE equilibrated with sediment contained a diverse set of HOCs with total concentrations in PE ranging from $0.05 \mathrm{mg} / \mathrm{g}_{\mathrm{PE}}$ (the reference site Maneer 5) to $101 \mathrm{mg} / \mathrm{g}_{\mathrm{PE}}$ (deep portion of Island End sediment, Table 5.2). The PE equilibrated with Maneer 5 contained slightly different total HOCs than a PE blank, which was extracted without being exposed to any sediment. The total HOC loading in the PE blank $\left(0.12 \mathrm{mg} / \mathrm{g}_{\mathrm{PE}}\right.$, Table 5.2) reflected both potential contamination present in PE or added during the extraction procedure (trace amounts of $n$ alkanes and $n$-alkenes, confirmed by GC $\times$ GC-TOFMS analysis, data not shown), as well as the average level of noise associated with integrating a blank-subtracted chromatogram. However, with the exception of Maneer 5, which was a reference site, the total HOC loadings in all the other sediment equilibrated PEs were 40 - 800 times higher than measured in the blank (Table 5.2), suggesting contamination and/or noise in the chromatogram could impact the calculation of the integrated HOC load at most by $0.1-1 \%$.

The patterns of HOCs in PE were generally conserved within sediments collected from one site, but different across the three major sampling sites (San Francisco Bay, Boston Harbor and Tabbs Bay). Most of the UCM in Island End PE eluted between alkanes with 12 to 37 carbon atoms ( $n \mathrm{C} 12-n \mathrm{C} 37, \sim 40-80 \mathrm{~min}$, Figure 5.4) while in $\mathrm{C} 1800 \mathrm{PE}$, the later eluting UCM ( $n \mathrm{C} 25-$ $n \mathrm{C} 35)$ dominated over the early portion $(n \mathrm{C} 15-n \mathrm{C} 22)$. In contrast, Tabbs Bay 8 PE contained only an early eluting $\mathrm{UCM}(n \mathrm{C} 15-n \mathrm{C} 22)$, with most of the mass concentrated between $n \mathrm{C} 12$ and $n \mathrm{C} 15$. The UCM patterns were conserved within sediments collected at the same site. For example, the PE incubated with deep Island End sediment (chromatogram not shown) had a similar distribution of HCs across the chromatogram, but contained total HOCs concentrations $3 \times$ higher than the PE incubated in top sediment from Island End (Table 5.2). 
Table 5.2 Total concentration of HOCs calculated based on GC $\times$ GC-FID chromatograms of PE equilibrated with various sediments, and corresponding model-estimated total concentration of HOCs in membrane lipids.

\begin{tabular}{lcc} 
Sample & $\begin{array}{c}\text { Concentration in PE } \\
\left(\mathrm{mg}_{\mathrm{HOCs}} / \mathrm{gPE}_{\mathrm{PE}}\right)\end{array}$ & $\begin{array}{c}\text { Concentration in lipid } \\
\left(\mathrm{mg}_{\mathrm{HOCs}} / \mathrm{g}_{\mathrm{PL}}\right)\end{array}$ \\
\hline Island End Top & 37 & 55 \\
Island End Deep & 101 & 145 \\
C1800 & 60 & 40 \\
303.1 & 1.3 & 2.4 \\
Tabbs Bay 10 & 2.4 & 2.3 \\
Tabbs Bay 8 & 5.0 & 6.0 \\
Maneer 5 & 0.23 & 0.07 \\
Blank & 0.12 & 0.06 \\
\hline
\end{tabular}

For all PE samples, the sum of EPA 16 PAHs $\left(\Sigma \mathrm{PAH}_{16}\right)$ was less than $10 \%$ of the total HOC load ( $2 \%$ for Island and $8 \%$ for 303.1, all other samples $<1 \%$, Figure 5.5). However, numerous other PAHs (methylated derivatives, and parent isomers) were present, particularly in Island End and 303.1 PEs (Figure 5.4, Figure 5.5). Further investigation with GC $\times$ GC- TOFMS revealed that this area of the chromatogram contained other polycyclic aromatic compounds such as dibenzothiophenes ( $\mathrm{m} / \mathrm{z}$ of 147$)$, dibenzofurans $(\mathrm{m} / \mathrm{z}$ of 168$)$, and their $\mathrm{C} 1$ and $\mathrm{C} 2$ derivatives. This extended (polyaromatic compounds) PAC fraction of the HOC mixture was calculated by integrating over an approximate area of the chromatogram defined to include all compounds eluting above the methylated series of the 16 PAHs ( $\mathrm{C} 1$ and $\mathrm{C} 2$, dashed line in Figure 5.4). The $\Sigma$ PAC concentration accounted for $20 \%$ of the total HOC load in both Island End PEs and for $30 \%$ in 303.1 PE. For Tabbs Bay PE, $\Sigma$ PACs only accounted for $\sim 10 \%$ of the total HOC load and less than $1 \%$ for the $\mathrm{C} 1800 \mathrm{PE}$. 

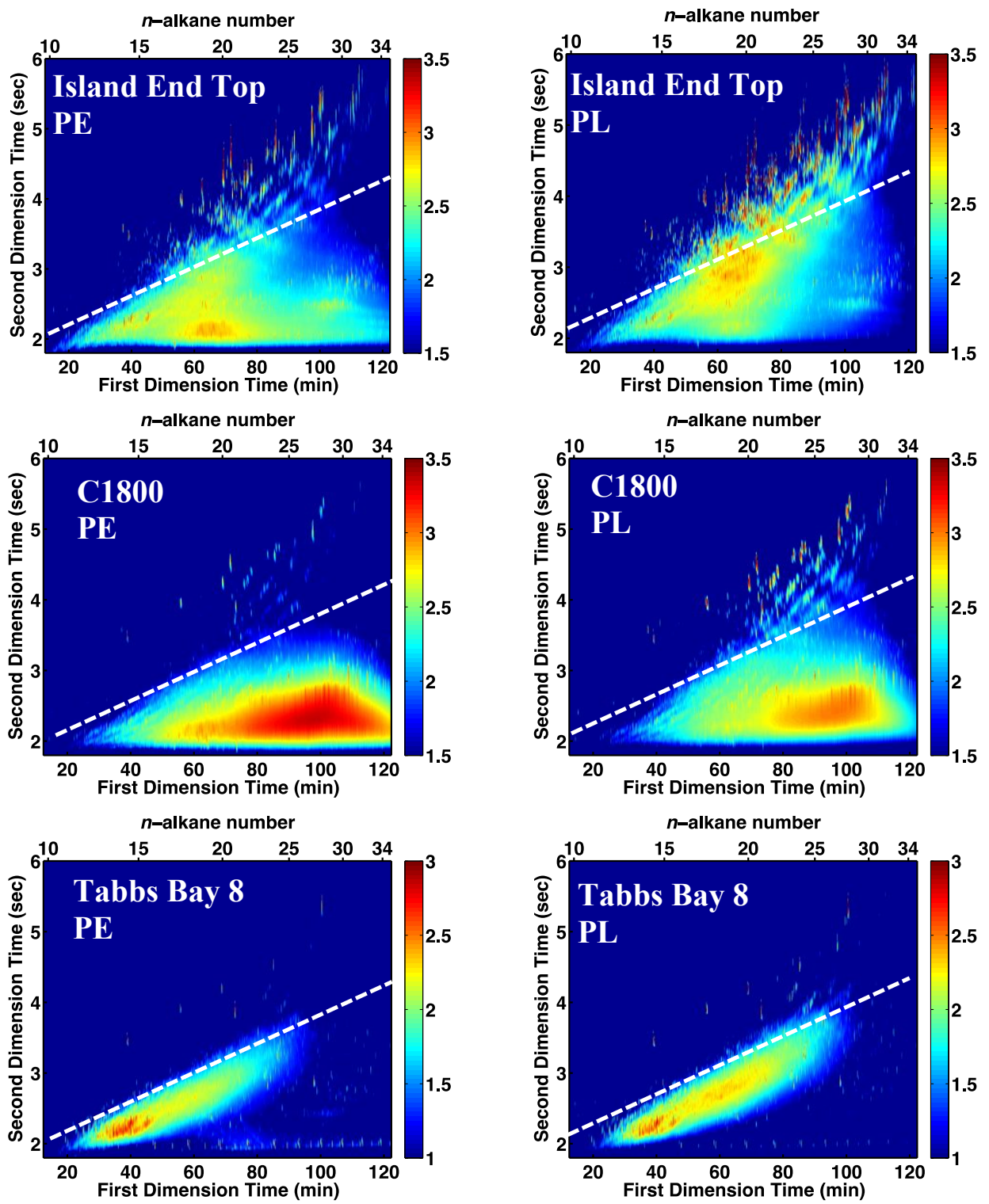

Figure 5.4. GC $\times$ GC-FID chromatograms of PE extracts (left) and corresponding modelestimated PL chromatograms (right) for Island End, C1800 and Tabbs Bay 8 test sediments, illustrating the different features of the HOC mixtures at each site. At each point in the chromatogram, the color can be directly interpreted as a $\log _{10}$ of the concentration $\left(\mathrm{ng} / \mathrm{g}_{\mathrm{PE}}\right.$ or $\mathrm{ng} / \mathrm{g}_{\mathrm{PL}}$ ) using the color bar on the right side of each panel. The area above the dashed white line refers to the portion of the chromatogram integrated for calculation of $\Sigma$ PACs. 

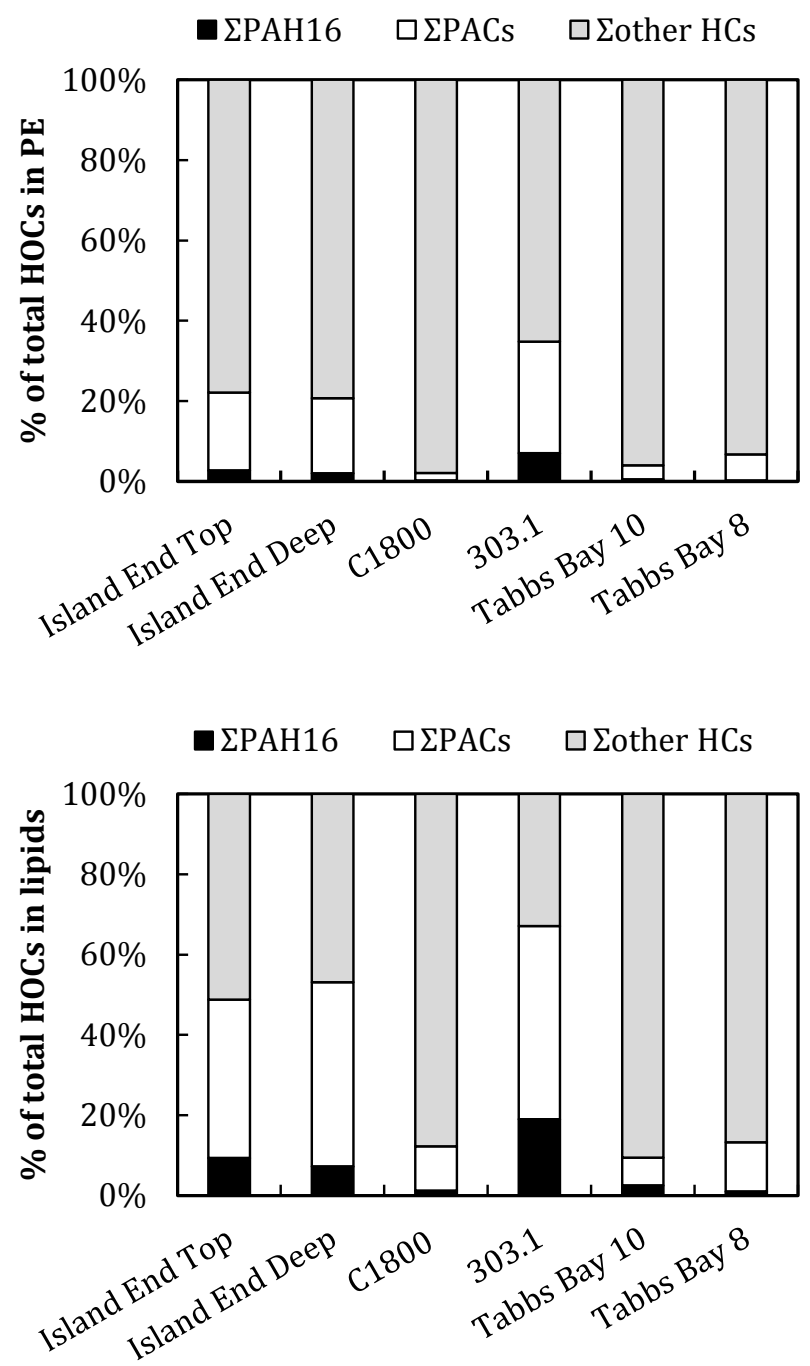

Figure 5.5 (top) Distribution of priority PAHs $(\Sigma \mathrm{PAH} 16)$, total PACs ( $\Sigma$ PACs) and other hydrocarbons (Eother HCs) in PE equilibrated with each test sediment (reference sediment Maneer 5 not shown). (bottom) Distribution of the same compound classes but for the GC $\times$ GCmodel predicted load of HOCs in the lipids of an organism at equilibrium with the PE.

Combining the PE chromatograms with equations for evaluating $K_{P E W}$ and $K_{P L W}$ from GC $\times$ GC retention times (Chapter 3, Equations 3.10 and 3.11), we calculated the concentration of all the HOCs in the lipids of an organism at equilibrium with the sediment. The model-estimated lipid loads differed from the measured PE loads on average by $25 \%$. The concentrations of PACs and the moderately polar components of the $\operatorname{UCM}\left(R T_{2}>3 \mathrm{~s}\right)$ were larger in the lipids than in the PE by factors of 2-3 (Figure 5.4 compare left and right panels). In contrast, the concentration of the saturated portion of the UCM (closer to $n$-alkane line, $R T_{2} 2-3 \mathrm{~s}$ ) was attenuated in lipids 
compared to PE by $\sim 10$ fold. As a consequence, for some sites that contained a large fraction of PAHs, the lipid loads were higher than the corresponding PE loads (e.g., Island End, 303.1, Table 5.2, Figure 5.4) whereas for non PAH-dominated mixtures the lipid loads were lower than the PE loads (e.g., C1800, Tabbs Bay 8, Table 5.2, Figure 5.4). Overall, the PACs accounted for a larger fraction of the total HOC load in the lipids than in PE by 2-6 fold (compare top and bottom of Figure 5.5).

Finally, combining $\mathrm{GC} \times \mathrm{GC}$ model-estimated $\mathrm{HOC}$ concentrations in lipids and the narcosis threshold identified in Figure 5.3 (20 mg/g lipid for 10\% immobilization), we can determine which of the tested sediments are likely to pose baseline toxicity health threats. The calculated body burdens due to the EPA 16 priority PAHs $\left(\Sigma \mathrm{PAH}_{16} 0.1-11 \mathrm{mg} / \mathrm{glipid}\right)$ were below the narcosis threshold for all PE samples. However, considering the concentration of all PAHs and including other aromatic heterocyclic (as defined in Figure 5.4), the narcosis threshold would be exceeded for the PE's equilibrated with the top and deep sections of Island End sediment

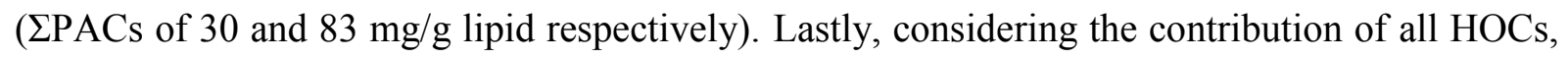
we would additionally expect the C1800 PE to show toxicity towards Daphnia magna (Table 5.2).

\subsubsection{Comparison between empirical and GC $\times$ GC-based estimations of toxicity}

When using the standard passive dosing protocol (5.2.5), immobilization of Daphnia magna was only observed for PE equilibrated with deep layer of Island End (50\%). However, the observed toxicity increased when a 3 day pre-equilibration time was used instead of the 1 day pre-equilibration of the standard protocol (Figure 5.6). With a 3 day pre-equilibration of the PE and test water prior to addition of Daphnia magna, both C1800 and Island End top PE showed measurable toxicity (38 and 72\%, respectively) and the measured toxicity of Island End Deep PE increased to $96 \%$. The PE incubated with the reference sediments Maneer 5 and 303.1 did not show significant toxicity with either 1 or 3 day pre-equilibrations. However, PE equilibrated with Tabbs Bay 8 was weakly toxic (35\% immobilization) when pre-equilibrated for 3 days, but not significantly toxic when pre-equilibrated for 1 day. 

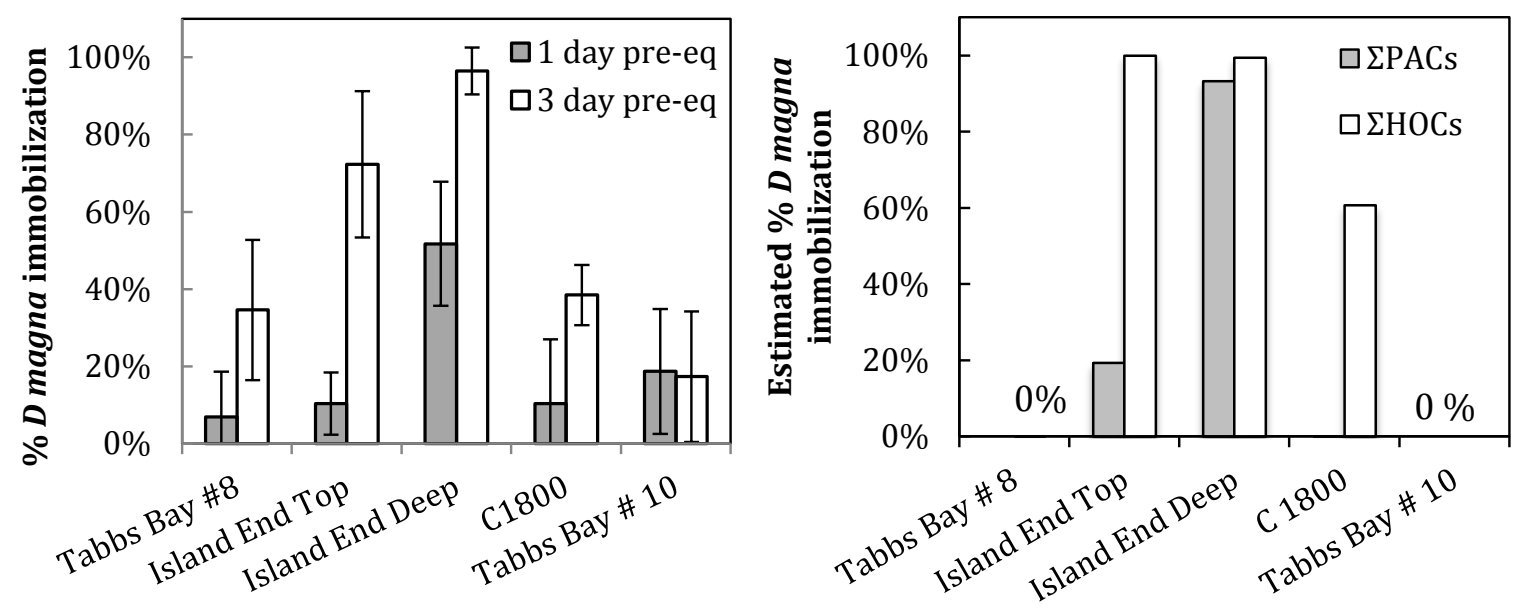

Figure 5.6. (left) Comparison between \% Daphnia magna immobilization observed using a 1 day and 3 day pre-equilibration time for PE and water before addition of test organisms. (right) Estimated Daphnia magna immobilization due to accumulation of the $\Sigma$ PACs or $\Sigma$ HOCs accumulation in lipids.

The observed toxicity using 3 day pre-equilibration matched the predictions of toxicity based on the dose response curve derived in Figure 5.3 quite well (Figure 5.7 note that line is not a best fit, but the dose response curve from Figure 5.3). The only PE which exhibited greater toxicity than expected based on the HOC concentrations was Tabbs Bay 8. Toxicity in the presence of PE equilibrated with Tabbs Bay 8 was previously observed in embryo development tests with the sea urchin Arbacia punctulata (77\% of normal development). ${ }^{47}$ While the embryo development process is known to be susceptible to membrane disruption from HOCs, the toxicity in the sea urchin assays was attributed to the presence of a specific chemical with a mode of action different than narcosis (i.e. the flame retardant tris(2-chloroethyl)phosphate (TCEP) LC50 of $140 \mathrm{mg} / \mathrm{L}$ for Daphnia magna). ${ }^{47}$ It is possible that the presence of TCEP in the PE could have caused the observed toxicity, and its PE-water equilibration kinetics is consistent with the increase in toxicity observed for the 3 day pre-equilibration time. The release of TCEP from PE would likely be membrane controlled because of its low $K_{O W}$ (as opposed to the release of phenanthrene which was WBL controlled), and assuming its membrane diffusivity, $D_{P E}$, was on the order of $10^{-10} \mathrm{~cm}^{2} / \mathrm{s}$ (similar diffusivity to endosulfan sulfate $D_{P E}$ of $10^{-9.6}$ due to similar molar volumes and presence of heteroatoms, Chapter 4), then the equilibration timescale for a $100 \mu \mathrm{m}$ membrane would be on the order of $3 \mathrm{~d}$. However, the presence of TCEP in the PE used 
in our experiments remains to be confirmed, and we cannot exclude contributions from other organic chemicals in the PE that with modes of action different than baseline toxicity.

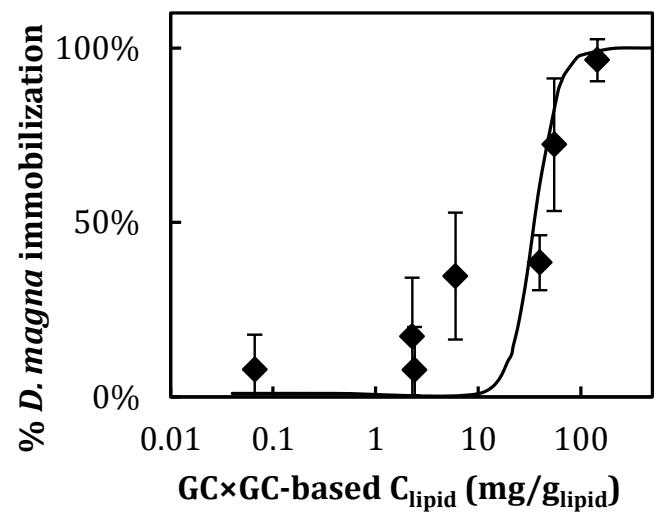

Figure 5.7. Observations of \% Daphnia magna immobilization (diamonds) versus estimated body burdens from GC $\times$ GC-based analysis of sediment-equilibrated PE. The line represents the dose response curve determined based on passive dosing experiments with individual and mixtures of test chemicals (e.g., PAHs, 1,3,5-TCB, see Figure 5.3).

The observed toxicity was poorly explained by considering only the cumulative narcosis potential of the 16 priority PAHs, which were below the narcosis threshold for all sedimentequilibrated PEs $\left(\mathrm{SPAH}_{16} 0.1-11 \mathrm{mg} / \mathrm{g}_{\text {lipid }}\right)$. Even when considering a broader range of PACs (including other $\mathrm{PAH}$ isomers and aromatic heterocycles, and their methylated derivatives, as defined by the line in Figure 5.4), the estimations of toxicity were still below the empirical determinations for Island End Top and C1800 PEs (estimated immobilization of $20 \%$ and $<1 \%$ compared to measured 72 and 38\%, respectively). For Island End Deep, the observed toxicity of $92 \%$ was in good agreement with the estimation based on $\Sigma$ PACs of $93 \%$ immobilization, supporting the findings of Hawthorne et al. ${ }^{17}$ that methylated PAHs (which form a large portion of the $\Sigma$ PACs) account for the majority of toxicity at coal tar contaminated sites. Interestingly, the estimations of toxicity based on $\Sigma$ PACs were closer to the observed toxicities using 1 day pre-equilibrations (10\% immobilization for both Island End top and C1800 PEs). This observation supports the hypothesis, which will be discussed in the following section, that a 1 day pre-equilibration may not have been enough for all mixture components to reach equilibrium concentrations in the water. 
It should be noted also that as expected from the historical contamination at the site, the C1800 PE did contain DDT as well as its degradation products DDE and DDD, and the potential for specific (non-baseline) toxicity effects of these three chemicals should be considered. Previous studies have measured the EC50 in $48 \mathrm{~h}$ Daphnia immobilization tests for DDT, ${ }^{48}$ $\mathrm{DDD}^{49}$ and $\operatorname{DDE}^{50}$ (1-2, 9 and $5 \mu \mathrm{g} / \mathrm{L}$, respectively). In our experiments, though, the aqueous concentrations of DDT, DDD and DDE calculated based on measured concentrations in the $\mathrm{C} 1800 \mathrm{PE}\left(<5 \mathrm{ng} / \mathrm{g}_{\mathrm{PE}}, \sim 8 \mu \mathrm{g} / \mathrm{g}_{\mathrm{PE}}\right.$ and $2 \mu \mathrm{g} / \mathrm{g}_{\mathrm{PE}}$, respectively), would be well below the effective concentrations identified previously $(<0.01,80$ and $3.2 \mathrm{ng} / \mathrm{L}$, respectively). In addition, the chemical activities corresponding to the measured EC50 values are consistent with Ea50 values measured for baseline toxicity (e.g. 0.03 for DDT), suggesting that at least in the $48 \mathrm{hr}$ Daphnia magna immobilization test, the toxicity of DDT, DDE and DDD is likely due to membrane disruption and not more specific effects.

\subsection{Discussion}

In general there was good agreement between the $\mathrm{GC} \times \mathrm{GC}$ based predictions of cumulative HOC loads in membrane lipids and empirical observations of baseline toxicity using passive dosing. The results supported our working hypothesis that baseline toxicity will be poorly predicted by the expected body burdens of priority pollutants alone (in this case $\Sigma \mathrm{PAH}_{16}$ ). However, questions remain regarding (1) the difference in observed toxicity between 1 day and 3 day equilibrated PE, and (2) the lack of toxicity of octadecane-loaded PE, and we consider these questions in the following sections from the perspective of kinetics and thermodynamics of HOCs partitioning between PE and membrane lipids.

\subsubsection{Kinetics of various $\mathrm{HOC}$ mixture components}

Based on the range of $K_{P E W}$ values $\left(10^{4}-10^{10}\right)$ calculated from $\mathrm{GC} \times \mathrm{GC}$ retention times, we estimate that the PE-water equilibration times for components of HOC mixtures, in static conditions, would be on the order of 1.5 to $3.5 \mathrm{hrs}$. As it was established in Chapter 4, the transfer of chemicals between PE and water exchange is WBL-controlled if the transport resistance through the boundary layer is smaller than the corresponding resistance through the membrane: 


$$
\frac{\mathrm{D}_{\mathrm{W}}}{\delta} \ll \frac{\mathrm{D}_{\mathrm{PE}} K_{P E W}}{L}
$$

where $D_{P E}$ and $D_{W}$ are the diffusivities in PE and water $\left(\mathrm{cm}^{2} / \mathrm{s}\right), \delta$ is the thicknesses of the WBL and $\mathrm{L}$ is half thickness of PE membrane. For a $100 \mu \mathrm{m}$ thick membrane and a WBL of $\sim 500 \mu \mathrm{m}$, chemicals with $K_{P E W}>10^{4}$ will be WBL controlled, assuming typical values of diffusivities in water $\left(10^{-6}-10^{-5} \mathrm{~cm}^{2} / \mathrm{s}\right)^{25}$ and in PE $\left(10^{-8}-10^{-10} \mathrm{~cm}^{2} / \mathrm{s}\right){ }^{41}$ For WBL controlled transport, the approach to equilibrium is characterized by a simple exponential (Equation 4.1), and the coefficient of the exponential is proportional to $\approx M W^{0.46}$ (the mass transfer coefficient is $\mathrm{D}_{\mathrm{W}} / \delta$, and $D_{W} \approx M W^{0.7}$ and $\delta \approx M W^{0.24}$, Chapter 4). For a molecular weight range of 100 to $500 \mathrm{~g} / \mathrm{mol}$, and using the measured equilibration time of phenanthrene ( $2 \mathrm{~h}$, Figure 5.1) as a reference, this gives us a range of equilibration times from 1.5 to $3.2 \mathrm{hrs}$. As a result, if most of the HOCs are in WBL regime, their concentration in the water should reach equilibrium by 1 day, even in static conditions.

The kinetics of PE-water mass transfer, as described by the model in Chapter 4, do not appear to explain the difference in observed toxicity between 1 and 3 days. Yet, the toxicity estimated based on the more polar components of the mixtures (which are expected to be behave more similarly to the PAHs) were closer to the 1 day measurements, which suggests that a larger fraction of the UCM could have reached equilibrium in 3 days compared to 1 day. In contrast, compounds eluting closer to the PAH line are likely going to have kinetics that are similar to that of phenanthrene and other PAHs whose PE-water kinetics have been studied more. Photoactivation was discarded as a likely explanation, because the equilibration was done in the dark and the increase in toxicity was observed for both PAH-rich and UCM-rich PEs. It is possible that photoactivation did occur during the 2 day exposure to Daphnia magna (which was performed with $12 \mathrm{~h}: 12 \mathrm{~h}$ light dark cycle), but in that case the 1 day and 3 day pre-equilibrations should have given the same measured toxicity.

The possibility that the PE-water transfer model formulated in Chapter 4 may not be applicable when dealing with sediment-equilibrated PE must also be considered. The loading of the PE at levels as high as was measured in Island End $\left(0.05-0.1 \mathrm{~g} / \mathrm{g}_{\mathrm{PE}}\right)$ could have changed the diffusivities in the polymer. For example, a 10 fold decrease in $D_{P E}$ for all compounds would lead to a range of compounds with $K_{P E W}$ between $10^{3}$ and $10^{5}$ being membrane controlled which 
as was discussed for TCEP (5.3.5) could lead to equilibration times of 1-3 days instead of a few hours. For example, compounds like dibenzothiophene and dibenzofuran, and their methylated derivatives, have lower $K_{P E W}$ and $D_{P E}$ values than their hydrocarbons counterparts, rendering the transport membrane controlled. Further analysis of the data will be required to establish if theses heterocyclic molecules form a large enough portion of the overall HOC load, such that their equilibration could lead to a difference in toxicity. Alternatively, the presence of organic films on the outside of PE could have also changed the equilibration kinetics.

\subsubsection{PE-lipid partitioning for various HOC mixture components}

As mentioned before, the $\mathrm{GC} \times \mathrm{GC}$ based model for partition coefficients suggests that differential partitioning will occur between lipid and PE as dictated by the compound's polarity. Compounds closer to the $n$-alkane line will prefer the PE by a factor of $\sim 10$, whereas compounds closer to in second retention time to the PAHs will favor lipids by approximately 6 fold. Considering this model result and looking at the data in Table 5.1, we can see that indeed one would not likely expect toxicity of octadecane PE because the loading was too low (translated into a lipid load of $4 \mathrm{mg} / \mathrm{g}$ below the threshold). The contradiction with the activity approach could mean that the PE was not loaded with octadecane at equilibrium due to kinetic limitations. Diffusion of such large $n$-alkanes in PE has not been studied in depth. It is possible that a fast initial uptake due to octadecane adsorbing to the surface of PE is followed by a slow step of octadecane diffusing in and reaching thermodynamic equilibrium, and the loading time was not sufficient to allow completion of the second step.

The differential partitioning of chemicals between PE and membrane lipid vesicles was studied independently in a preliminary partitioning experiment using DMPC vesicles and PE loaded with various test chemicals (5.2.10). The results of the experiment confirmed that polar chemicals (phenanthrene and 1,3,5 TCB) preferred the lipid over the PE phase, while non polar ones like octadecane and $n$-tetradecylbenzene strongly preferred the PE phase (Figure 5.8). The DMPC-PE partition coefficients measured for phenanthrene and 1,3,5-TCB $\left(K_{D M P C-P E}\right.$ of $8.7 \pm$ 0.8 and $2.8 \pm 0.4$, respectively) were similar to those predicted from $\mathrm{GC} \times \mathrm{GC}$ estimates (6.3 and 3.2 , respectively). For octadecane and $n$-tetradecylbenzene, the GC $\times$ GC-based $K_{P L-P E}$ were larger than the values for DMPC-PE partitioning measured experimentally by a factor of $\sim 10$. However, the experimental determinations of DMPC-PE partition coefficients for octadecane 
and $n$-tetradecylbenzene $(0.015 \pm 0.005$ and $0.06 \pm 0.05$, respectively) had more uncertainty than for phenanthrene and 1,3,5-TCB because the fractional losses from PE was only $\sim 20 \%$. The partitioning experiments did support, though, the trend inferred from $\mathrm{GC} \times \mathrm{GC}$ retention times that aliphatic and monoaromatic compounds will partition more into PE compared to lipids. Combining the $K_{P L-P E}$ value of octadecane with its measured concentration in PE at saturation further reinforces the argument that if the Daphnia magna tissue did reach chemical equilibrium with the PE, the dose of octadecane was not high enough to cause toxicity. Lastly, it is worth noting that the experimental determinations of $K_{P L-P E}$ was done for DMPC vesicles, whereas the GC $\times$ GC-based relationship was trained on $K_{P L W}$ coefficients averaged across lipids with the same polar headgroup but different side chains (e.g., 1-palmitoyl-2-oleoyl-sn-glycero-3phosphocholine, POPC, egg PC and soy PC).

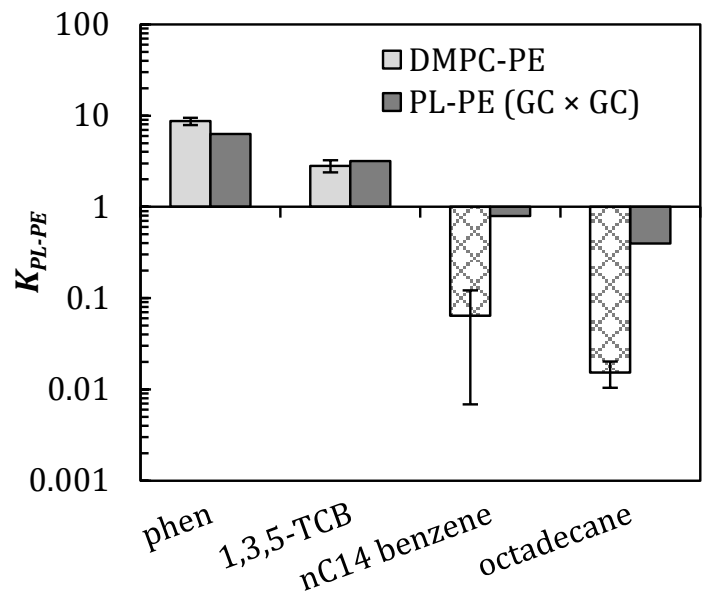

Figure 5.8 Experimentally measured partition coefficients between DMPC and PE (DMPC-PE) and $\mathrm{GC} \times \mathrm{GC}$-estimated PL-PE $(\mathrm{PL}-\mathrm{PE}(\mathrm{GC} \times \mathrm{GC}))$ partition coefficients for phenanthrene, 1,3,5-TCB, $n \mathrm{C} 14$ benzene and octadecane. Experimental values were determined at room temperature. The pattern fill denotes measurements that are expected to have more uncertainty due to small loss from PE to the lipid phase.

\subsection{Conclusions}

In this study, we implemented a PE-based passive dosing protocol for testing the baseline toxicity of HOC mixtures. The protocol was validated by measuring the dose response curves of phenanthrene and 1,3,5-TCB, which are both known to cause baseline toxicity. The EC50s for 
both of these chemicals were in good agreement with previously published values. ${ }^{22,45}$ Passive dosing with sediment-equilibrated PE was then used to measure toxicity of environmental HOC mixtures. The toxicity of HOC mixtures was consistent with estimations of lipid body burdens based on GC $\times$ GC-FID analysis and an equilibrium partition model parameterized with $K_{P E W}$ and $K_{P L W}$ partition coefficients calculated from the two dimension retention times. More research is needed to understand the kinetics of PE-water equilibration for sediment-exposed PE, as it remains to be determined why the toxicity observations were different with 1 versus 3 day preequilibrations.

The GC $\times$ GC-derived partition coefficients suggested that the more polar components of a an HOC mixture (i.e. PAHs) have a greater affinity to lipids and would contribute more towards body burdens than the more saturated, aliphatic components. The toxicity of PE equilibrated with coal tar contaminated sediment did appear to come mostly from the PAH load, while for C1800, the toxicity appeared to come mostly from UCM. Across all the contaminated sediments, the baseline toxicity was poorly predicted when only the EPA 16 PAHs were considered, suggesting that cumulative toxicity due to these compounds only is not a good metric at sites where other aromatic compounds (e.g. methylated derivatives of PAH or heterocyclic aromatic compounds) are present.

\section{Acknowledgements}

We thank John MacFarlane and Josefin Betsholtz for assistance with Island End sediment collection, Guilherme Lotufo for providing the C1800 and 303.1 sediments, and to collaborators Marion Nipper and Scot Carr for collecting the Tabbs Bay sediment. LSR acknowledges support from the UROP Office at MIT. 


\section{References}

1.Verhaar, H. J. M.; Vanleeuwen, C. J.; Hermens, J. L. M., Classifying Environmental-Pollutants .1. Structure-Activity-Relationships for Prediction of Aquatic Toxicity. Chemosphere 1992, 25, (4), 471-491.

2.McCarty, L. S.; Mackay, D.; Smith, A. D.; Ozburn, G. W.; Dixon, D. G., Residue-based interpretation of toxicity and bioconcentration QSARs from aquatic bioassays - neutral narcotic organics. Environ Toxicol Chem 1992, 11, (7), 917-930.

3.Sikkema, J.; de Bont, J. A.; Poolman, B., Mechanisms of membrane toxicity of hydrocarbons. Microbiol Rev 1995, 59, (2), 201-22.

4.Konemann, H., Quantitative structure-activity relationships in fish toxicity studies. Part 1: relationship for 50 industrial pollutants. Toxicology 1981, 19, (3), 209-21.

5.McGrath, J. A.; Parkerton, T. E.; Hellweger, F. L.; Di Toro, D. M., Validation of the narcosis target lipid model for petroleum products: Gasoline as a case study. Environ Toxicol Chem 2005, $24,(9), 2382-2394$.

6.Di Toro, D. M.; McGrath, J. A.; Hansen, D. J., Technical basis for narcotic chemicals and polycyclic aromatic hydrocarbon criteria. I. Water and tissue. Environ Toxicol Chem 2000, 19, (8), 1951-1970.

7.Smith, E.; Wraige, E.; Donkin, P.; Rowland, S., Hydrocarbon humps in the marine environment: Synthesis, toxicity, and aqueous solubility of monoaromatic compounds. Environ Toxicol Chem 2001, 20, (11), 2428-2432.

8.Scarlett, A.; Rowland, S. J.; Galloway, T. S.; Lewis, A. C.; Booth, A. M., Chronic sublethal effects associated with branched alkylbenzenes bioaccumulated by mussels. Environ Toxicol Chem 2008, 27, (3), 561-567.

9.Du, J.; Mehler, W. T.; Lydy, M. J.; You, J., Toxicity of sediment-associated unresolved complex mixture and its impact on bioavailability of polycyclic aromatic hydrocarbons. Journal of Hazardous Materials 2012, 203-204, (0), 169-175.

10.Altenburger, R.; Greco, W. R., Extrapolation concepts for dealing with multiple contamination in environmental risk assessment. Integr Environ Assess Manag 2009, 5, (1), 628 .

11.Backhaus, T.; Faust, M., Predictive Environmental Risk Assessment of Chemical Mixtures: A Conceptual Framework. Environ Sci Technol 2012, 46, (5), 2564-2573.

12.Venturini, N.; Tommasi, L. R.; Bícego, M. C.; Martins, C. C., Characterization of the benthic environment of a coastal area adjacent to an oil refinery, Todos os Santos Bay (NE-Brazil). Brazilian Journal of Oceanography; Vol 52, No 2 (2004) 2004.

13.Booth, A. M.; Sutton, P. A.; Lewis, C. A.; Lewis, A. C.; Scarlett, A.; Chau, W.; Widdows, J.; Rowland, S. J., Unresolved complex mixtures of aromatic hydrocarbons: Thousands of overlooked persistent, bioaccumulative, and toxic contaminants in mussels. Environ Sci Technol 2007, 41, (2), 457-464.

14.Donkin, P.; Smith, E. L.; Rowland, S. J., Toxic effects of unresolved complex mixtures of aromatic hydrocarbons accumulated by mussels, Mytilus edulis, from contaminated field sites. Environ Sci Technol 2003, 37, (21), 4825-4830.

15.Martins, L. K. P.; Nascimento, I. A.; Fillmann, G.; King, R.; Evangelista, A. J. A.; Readman, J. W.; Depledge, M. H., Lysosomal responses as a diagnostic tool for the detection of chronic petroleum pollution at Todos os Santos Bay, Brazil. Environ Res 2005, 99, (3), 387-396. 
16.Echeveste, P.; Dachs, J.; Berrojalbiz, N.; Agusti, S., Decrease in the abundance and viability of oceanic phytoplankton due to trace levels of complex mixtures of organic pollutants. Chemosphere 2010, 81, (2), 161-168.

17.Hawthorne, S. B.; Miller, D. J.; Kreitinger, J. P., Measurement of total polycyclic aromatic hydrocarbon concentrations in sediments and toxic units used for estimating risk to benthic invertebrates at manufactured gas plant sites. Environ Toxicol Chem 2006, 25, (1), 287-296.

18.Lin, Z. F.; Du, J. W.; Yin, K. D.; Wang, L. S.; Yu, H. X., Mechanism of concentration addition toxicity: they are different for nonpolar narcotic chemicals, polar narcotic chemicals and reactive chemicals. Chemosphere 2004, 54, (11), 1691-1701.

19.Smith, K. E.; Schmidt, S. N.; Dom, N.; Blust, R.; Holmstrup, M.; Mayer, P., Baseline toxic mixtures of non-toxic chemicals: "solubility addition" increases exposure for solid hydrophobic chemicals. Environ Sci Technol 2013, 47, (4), 2026-33.

20.Engraff, M.; Solere, C.; Smith, K. E. C.; Mayer, P.; Dahllof, I., Aquatic toxicity of PAHs and PAH mixtures at saturation to benthic amphipods: Linking toxic effects to chemical activity. Aquatic Toxicol 2011, 102, (3-4), 142-149.

21.Rojo-Nieto, E.; Smith, K. E. C.; Perales, J. A.; Mayer, P., Recreating the seawater mixture composition of HOCs in toxicity tests with Artemia franciscana by passive dosing. Aquatic Toxicol 2012, 120, 27-34.

22.Smith, K. E. C.; Dom, N.; Blust, R.; Mayer, P., Controlling and maintaining exposure of hydrophobic organic compounds in aquatic toxicity tests by passive dosing. Aquatic Toxicol 2010, 98, (1), 15-24.

23.Ferguson, J., The Use of Chemical Potentials as Indices of Toxicity. Proceedings of the Royal Society of London B: Biological Sciences 1939, 127, (848), 387-404.

24.Mayer, P.; Reichenberg, F., Can highly hydrophobic organic substances cause aquatic baseline toxicity and can they contribute to mixture toxicity?

25.Schwarzenbach, R. P.; Gschwend, P. M.; Imboden, D. M., Environmental Organic Chemistry. 2nd ed.; John Wiley: New York, 2003.

26.Kraaij, R.; Seinen, W.; Tolls, J., Direct evidence of sequestration in sediments affecting the bioavailability of hydrophobic organic chemicals to benthic deposit-feeders. Environ Sci Technol 2002, 36, (16), 3525-3529.

27.Hawthorne, S. B.; Azzolina, N. A.; Neuhauser, E. F.; Kreitinger, J. P., Predicting Bioavailability of sediment polycyclic aromatic hydrocarbons to Hyalella azteca using equilibrium partitioning, Supercritical fluid extraction, and pore water concentrations. Environ Sci Technol 2007, 41, (17), 6297-6304.

28.Reichenberg, F.; Mayer, P., Two complementary sides of bioavailability: accessibility and chemical activity of organic contaminants in sediments and soils. Environ Toxicol Chem 2006, $25,(5), 1239-45$.

29.TPHCWG, Analysis of Petroleum Hydrocarbons in Environmental Media. In Weisman, W., Ed. Amherst Scientific Publishers: Amherst, MA, 1998; Vol. 1.

30.Verbruggen, E. M. J.; Beek, M.; Pijnenburg, J.; Traas, T. P., Ecotoxicological environmental risk limits for total petroleum hydrocarbons on the basis of internal lipid concentrations. Environmental Toxicology And Chemistry / SETAC 2008, 27, (12), 2436-2448.

31.Muijs, B.; Jonker, M. T. O., Assessing the Bioavailability of Complex Petroleum Hydrocarbon Mixtures in Sediments. Environ Sci Technol 2011, 45, (8), 3554-3561.

32.Greenberg, M. S.; Chapman, P. M.; Allan, I. J.; Anderson, K. A.; Apitz, S. E.; Beegan, C.; Bridges, T. S.; Brown, S. S.; Cargill, J. G. t.; McCulloch, M. C.; Menzie, C. A.; Shine, J. P.; 
Parkerton, T. F., Passive sampling methods for contaminated sediments: risk assessment and management. Integr Environ Assess Manag 2014, 10, (2), 224-36.

33.Gros, J.; Reddy, C. M.; Aeppli, C.; Nelson, R. K.; Carmichael, C. A.; Arey, J. S., Resolving Biodegradation Patterns of Persistent Saturated Hydrocarbons in Weathered Oil Samples from the Deepwater Horizon Disaster. Environ Sci Technol 2014, 48, (3), 1628-1637.

34.Kwon, H.-C.; Roh, J.-Y.; Lim, D.; Choi, J.; Kwon, J.-H., Maintaining the constant exposure condition for an acute Caenorhabditis elegans mortality test using passive dosing. Environmental health and toxicology 2011, 26, e2011015-e2011015.

35.ASTM E2591-07 Standard Guide for Conducting Whole Sediment Toxicity Tests with Amphibians; ASTM International: West Conshohocken, PA, 2013; 10.1520/E2591-07R13.

36.ASTM E729-96 Standard Guide for Conducting Acute Toxicity Tests on Test Materials with Fishes, Macroinvertebrates, and Amphibians; ASTM International: West Conshohocken, PA, 2014.

37.Smith, K. E.; Oostingh, G. J.; Mayer, P., Passive dosing for producing defined and constant exposure of hydrophobic organic compounds during in vitro toxicity tests. Chem Res Toxicol 2010, 23, (1), 55-65.

38.Allan, S. E.; Smith, B. W.; Tanguay, R. L.; Anderson, K. A., Bridging environmental mixtures and toxic effects. Environ Toxicol Chem 2012, 31, (12), 2877-87.

39.Endo, S.; Escher, B. I.; Goss, K. U., Capacities of membrane lipids to accumulate neutral organic chemicals. Environ Sci Technol 2011, 45, (14), 5912-21.

40.CSID:11145. http://www.chemspider.com/Chemical-Structure.11145.html (19:35, Mar 19, 2015)

41.Lohmann, R., Critical review of low-density polyethylene's partitioning and diffusion coefficients for trace organic contaminants and implications for its use as a passive sampler. Environ Sci Technol 2012, 46, (2), 606-18.

42.Nabi, D.; Gros, J.; Dimitriou-Christidis, P.; Arey, J. S., Mapping environmental partitioning properties of nonpolar complex mixtures by use of GC x GC. Environ Sci Technol 2014, 48, (12), 6814-26.

43.Vaes, W. H.; Ramos, E. U.; Hamwijk, C.; van Holsteijn, I.; Blaauboer, B. J.; Seinen, W.; Verhaar, H. J.; Hermens, J. L., Solid phase microextraction as a tool to determine membrane/water partition coefficients and bioavailable concentrations in in vitro systems. Chem Res Toxicol 1997, 10, (10), 1067-72.

44.Brown, R. S.; Akhtar, P.; Akerman, J.; Hampel, L.; Kozin, I. S.; Villerius, L. A.; Klamer, H. J. C., Partition controlled delivery of hydrophobic substances in toxicity tests using poly(dimethylsiloxane) (PDMS) films. Environ Sci Technol 2001, 35, (20), 4097-4102.

45.Ramamoorthy, S. R., S; Chlorinated Organic Compounds in the Environment. Regulatory and Monitoring Assessment. CRC Press: 1997; p 112.

46.Ventura, G. T.; Kenig, F.; Reddy, C. M.; Frysinger, G. S.; Nelson, R. K.; Mooy, B. V.; Gaines, R. B., Analysis of unresolved complex mixtures of hydrocarbons extracted from Late Archean sediments by comprehensive two-dimensional gas chromatography ( $\mathrm{GC} \times \mathrm{GC})$. Org Geochem 2008, 39, (7), 846-867.

47.Nipper, M. Field Validation of General Methodology for Evaluating Narcosis Toxicity using the Sediment Profile Imaging and Micro-sampling System (SPIMS) The NOAA/UNH Cooperative Institute for Coastal and Estuarine Environmental Technology (CICEET) 2011. http://ciceet.unh.edu/news/releases/spring11_reports/pdf/nipper_etd07.pdf 
48.Stratton, G.; Giles, J., Importance of bioassay volume in toxicity tests using algae and aquatic invertebrates. B Envion Contam Toxicol 1990, 44, (3), 420-427.

49.Montgomery, J. H., Groundwater Chemicals Desk Reference 3rd ed.; CRC Press: 2010; p 289.

50.Bettinetti, R.; Croce, V.; Noe, F.; Ponti, B.; Quadroni, S.; Galassi, S., Ecotoxicity of pp'DDE to Daphnia magna. Ecotoxicology 2013, 22, (8), 1255-63. 



\section{Chapter 6.}

\section{Measuring in-situ degradation rates of DDT with passive sampling}

A. Patricia Tcaciuc ${ }^{1,2 *}$, Raffaella Borrelli ${ }^{3}$, Luciano M. Zaninetta ${ }^{4}$, Philip M. Gschwend ${ }^{1,2}$

1MIT/WHOI Joint Program in Chemical Oceanography

2Department of Civil and Environmental Engineering, Massachusetts Institute of Technology, Cambridge, MA

${ }^{3}$ ENI Renewable Energy \& Environmental R\&D - Donegani Institute, Novara, Italy

${ }^{4}$ Syndial, San Donato Milanese, Italy 


\section{Abstract}

Passive sampling is becoming a widely used tool for assessing freely dissolved concentrations of hydrophobic organic contaminants in sediments and in the water column. The loss of performance reference compounds (PRCs), loaded in the sampler before deployment, is typically used to assess the fractional equilibration of target analytes. The key assumption behind the use of PRCs is that their release is diffusion driven. In this paper we show that when applying passive sampling for measuring reactive compounds such as DDT, transformations of PRCs in the sediment can enhance the release of those PRCs. In a PRC-loaded PE deployed at the sediment water interface of a freshwater lake, the loss of the ${ }^{13} \mathrm{C}-2,4$ '-DDT PRC was accelerated compared to the loss of other PRCs $\left({ }^{13} \mathrm{C}\right.$-labeled PCBs, as well as ${ }^{13} \mathrm{C}$-labeled DDE and DDD). The DDT PRC loss was also accompanied by an accumulation of its degradate, ${ }^{13} \mathrm{C}-2,4$ '-DDD in the PE. The field observations were also found in static laboratory incubations with fieldcollected sediment. Using a 1D reaction-diffusion model, we deduced the in situ degradation rates of DDT, based on the measured PRC loss. The values for in situ degradation rates increased with depth into the sediment bed $\left(0.09 \mathrm{~d}^{-1}\right.$ at $0-10 \mathrm{~cm}$ and $0.9 \mathrm{~d}^{-1}$ at $\left.30-40 \mathrm{~cm}\right)$, and compared favorably with values previously reported in the literature. This work shows that while the traditional interpretation of symmetric PRC loss and target accumulation applies to DDE and DDD, it may not apply to DDT, and by inference other compounds, when in situ transformation rates are comparable to rates of sampler-environment diffusive exchange. 


\subsection{Introduction}

The use of the insecticide, DDT, decreased sharply after several countries recognized its harmful effects and banned it in 1970s. Yet, decades later, DDT contamination persists in various parts of the world, ${ }^{1}$ and at levels that pose health risks to aquatic life and humans. ${ }^{2,3,4}$ At sites of concern, the risk associated with this chemical depends on its availability for transport or uptake into organisms, which is in turn related to its freely dissolved concentration (Lydy et al. ${ }^{5}$ and references therein).

Unlike persistent pollutants like PCBs (which can still be degraded in anaerobic sedimentary environments, but on timescales of several years to several decades ${ }^{6}$ ), DDT is subject to chemical transformations upon its release to aquatic environments, producing two major degradation products, DDE and DDD (altogether referred to as DDXs). The degradation of DDT in sediments is typically faster than that of PCBs, and it has been reported to occur with half lives from days ${ }^{7,8}$ to years, ${ }^{9,} 10$ depending on conditions in the sediment (e.g. $\mathrm{pH}$, redox potential, microbial communities). In addition, the DDE and DDD degradates are also susceptible to transformations. ${ }^{9,1}$ Therefore, modeling the long term fate of $\sum$ DDX (taken here to mean DDT, DDE and DDD together) in contaminated bodies of water requires information, not only about their freely dissolved concentrations, but also about their transformation rates, preferably tuned to in situ conditions.

Recent efforts to characterize pore water concentrations of DDXs in sediments have employed passive sampling in conjunction with performance reference compounds (PRCs). The PRC loss measured during the deployment time can be used to calculate the extent of disequilibrium of the analytes of interest. PRCs have been used successfully to determine porewater concentrations of PCBs ${ }^{12,13}$ and PAHs, ${ }^{14}$ as well as of DDE and DDD. ${ }^{15,16}$ However, at sites where DDT was present, previous work ${ }^{17,18}$ measured a near complete loss of the DDT PRC which was not accompanied by a corresponding uptake of the DDT target analyte. Similarly, while applying solid-phase microextraction (SPME) passive sampling to sediments spiked with DDT, Bao et al. ${ }^{15}$ found that the parent compound DDT spike was stable in some of the sediments used in their study, but not in others.

These studies suggest that the behavior of DDT-PRCs may not be fully understood, and this can have implications for the interpretation of passive sampler data. For example, if biotic or abiotic transformation of DDT is occurring in the sediment, then at least theoretically, these 
reactive processes can lead to a faster loss of PRCs than can be explained by diffusive transfers alone. In such instances, complete loss of the PRC may not correspond to complete equilibration of the target compound. Furthermore, if the degradation product of the DDT-PRC is already present in the sampler as a PRC (e.g., a labeled DDE or DDD), then this can change the rate at which the second PRC diffuses out of the passive sampler. Lastly, it is worth noting that understanding contaminant degradation is relevant, not only for the accurate interpretation of any passive sampling exercise, but also for the long term modeling of the fate of contaminants of interest. In the case of DDT, degradation rates can be important inputs in mass balance models aiming to compare remediation scenarios against natural attenuation.

The goals of this study where to: (1) determine if reactive processes can impact the transfer of PRCs and target chemicals between sediments and passive samplers, (2) build a reactiondiffusion mass transfer model which could be used to determine in situ degradation rates based on the measured PRC loss, and (3) investigate implications for passive sampling applications to reactive compounds. In this paper we show that using a combination of labeled PCBs and DDXs as PRCs, we can identify patterns in the PRC loss that are consistent with reaction-diffusion transport of the DDT-PRC. These patterns were present in field-deployed polyethylene (PE) samplers as well as in static laboratory incubations. Lastly, DDT reactivity in the test sediments was confirmed with incubations of sediment slurries with a labeled DDT substrate.

\subsubsection{PE/sediment bed 1D diffusion model.}

Applying Fick's law to a PE membrane in a unmixed sediment bed, and employing the method of Laplace transforms, Fernandez et al. (2009) ${ }^{14}$ developed a model for the exchange kinetics of chemicals between polymer membranes and static sediment beds. According to this model, a typical PRC loss curve is characterized by a fast initial decrease in PRC concentration, followed by a slow decline as diffusion through the sediment bed becomes increasingly limiting. The diffusion rate into the sediment bed depends in turn on the fraction of the chemical present in the porewater, and the sediment's porosity and tortuosity. Since porosity can be measured and tortuosity can be approximated based on porosity, ${ }^{14}$ the PRC transport can be ultimately modeled as dependent on $K_{d}$, the sediment/water partition coefficient. Lastly, for PRC and target compounds differing only in isotopic label, the fractional equilibration of a target chemical should be, at any time, equal to the fraction of PRC lost from the PE. 


\subsubsection{Development of 1D reaction-diffusion model.}

Assuming that degradation of a target compound and/or PRC takes place everywhere in the sediment bed, we can write the following governing equations:

$$
\begin{aligned}
& \frac{\partial C_{P E}}{d t}=D_{P E} \frac{\partial^{2} C_{P E}}{\partial x^{2}} \\
& \frac{\partial C_{S E D}}{d t}=D_{S E D} \frac{\partial^{2} C_{S E D}}{\partial x^{2}}-k_{r} C_{S E D}
\end{aligned}
$$

where $C_{P E}$ and $C_{S E D}$ are the concentrations $\left(\mathrm{mol} / \mathrm{cm}^{3}\right)$ in the PE and sediment, respectively, $D_{P E}$ and $D_{S E D}$ are the diffusivities in the PE and in sediment $\left(\mathrm{cm}^{2} \mathrm{~s}^{-1}\right)$, and $k_{r}$ is the first order degradation rate of the chemical in the sediment $\left(\mathrm{s}^{-1}\right)$. Using the same boundary and initial conditions as in the case of the diffusion-only model, and applying the Laplace transform method (SI-1) leads to the following Laplace space solutions for the concentration of PRC in PE:

$$
C_{P E}^{P R C}=C_{P E}^{P R C, 0}\left(\frac{1}{s}-\frac{1}{s^{\frac{3}{2}}\left(\operatorname{coth}(\sqrt{s})+\frac{K_{P E S E D} \sqrt{s}}{\sqrt{\psi} \sqrt{s+\overline{k_{r}}}}\right)}\right)
$$

where $s$ is the Laplace space parameter, $K_{P E S E D}$ is the PE-sediment partition coefficient $\left(\mathrm{L}_{\mathrm{SED}} / \mathrm{L}_{\mathrm{PE}}\right), \Psi$ is the ratio of diffusivities $\left(D_{S E D} / D_{P E}\right)$, and $\overline{k_{r}}$ is the reaction rate in nondimensional units $\left(=k_{r} L^{2} / D_{P E}\right)$. For a target chemical diffusing into PE and reacting in the sediment bed, the concentration in PE is given by:

$$
C_{P E}^{\text {target }}=C_{S E D}^{\text {target, } 0} K_{P E S E D}\left(\frac{1}{\sqrt{s}\left(s+\overline{k_{r}}\right)\left(\operatorname{coth}(\sqrt{s})+\frac{K_{P E S E D} \sqrt{s}}{\sqrt{\psi} \sqrt{s+\overline{k_{r}}}}\right)}\right)
$$

where $C_{S E D}^{\text {target, } 0}$ is the concentration in the sediment at the time of deployment. For $k_{r}=0$, both solutions (for PRC and target chemicals) reduce to the diffusion-only solutions. ${ }^{14}$ However, the sum of fractional PRC remaining and target equilibration is different from 1 in the time domain (or $1 / \mathrm{s}$ in Laplace space), consistent with the intuitive expectation that the uptake and release profiles will not be symmetric for reactive chemicals. The inversion of Equations 3 and 4 to time domain can be performed using Matlab or other programming languages, ${ }^{19}$ using code similar to one already published. ${ }^{14,20}$ Lastly, we note that the transport model of Fernandez et al. ${ }^{14}$, as well as the reaction-diffusion model developed here, is built using a volume-based $K_{d}\left(\mathrm{~L}_{\mathrm{sed}} / \mathrm{L}_{\mathrm{w}}\right)$. 
However, throughout this manuscript, the mass based $K_{d}\left(\mathrm{~kg}_{\mathrm{sed}} / \mathrm{L}_{\mathrm{W}}\right)$ is discussed, which was calculated from $K_{d}{ }^{\prime}$ according to the following formula:

$$
K_{d}=\frac{K_{d}^{\prime}}{(1-\phi) \rho_{s}}
$$

where $\phi$ is the porosity of the sediment and $\rho_{\mathrm{s}}$ is the density of the solids (assumed to be 2.5 $\mathrm{kg} / \mathrm{L})$.

\subsection{Materials and Methods}

Solvents used for extraction of PE and sediment were purchased from VWR (JT Baker Ultraresi-analyzed). Standards for labeled PCBs and DDTs were purchased from Cambridge Isotope Laboratory (all ${ }^{13} \mathrm{C}$ labeled chemicals and $\mathrm{d}_{8} 4,4$ '-DDD, Tewskbury, MA) and $\mathrm{C} / \mathrm{D} / \mathrm{N}$ Isotopes ( $\mathrm{d}_{8}$ 2,4'-DDT and $\mathrm{d}_{8}$ 4,4'-DDT, Pointe-Claire, Quebec). Polyethylene strips used as samplers in the field and in laboratory experiments, were cut from PE sheets $(25 \mu \mathrm{m}$, Film Guard 1 mil plastic drop cloth, Covalence Plastics, Minneapolis, MN), and cleaned by overnight submersions in dichloromethane, methanol, and Milli-Q water (Aries Vaponics, Rockland, MA).

\subsubsection{PRC loading of PE.}

The PE was loaded with PRCs by shaking on a rotary shaker table at $100 \mathrm{rpm}$ for $7 \mathrm{~d}$ in an 80:20 methanol:water mixture. ${ }^{21}$ The PE used in field and laboratory (static jar and tumbling slurries) incubations was loaded with the same PRCs but at different concentrations (Table S1).

\subsubsection{Field site overview}

Sampling was conducted at Lake Maggiore (Italy), a subalpine lake situated between Italy and Switzerland. Between 1948 and 1996, a plant producing technical grade DDT discharged waste in the River Toce, ${ }^{22}$ which flows into Lake Maggiore. This discharge led to DDT contamination of the lake, and particularly of the sediments of Pallanza Bay, located at the mouth of the river. The application of passive sampling at Lake Maggiore was interesting for two reasons. Firstly, in situ sampling for DDE and DDD would allow evaluation of the hazards associated with these chemicals in the sediments, as discussed elsewhere. ${ }^{18}$ Secondly, the site was ideal for studying reactive processes leading to DDT degradation as previous studies 
identified the presence of DDE and DDD degradation products of DDT in the sediments and biota. $^{22}$

The sampling activities of this study were conducted in the vicinity of Pallanza Bay, where in June 2013, we deployed passive samplers loaded with PRCs (Section 6.2.3) and collected sediment samples via grab samplers (Section 6.2.3), which were then used for experiments in the laboratory (Section 6.2.4). In addition to sediment samples collected during this field trip (labeled as F1 and F2, Section 6.2.3), two additional sediment samples, CARM and LM were used in laboratory incubations. These two sediments were collected during a field trip to Lake Maggiore in 2010, from a less contaminated site in the northern part of Lake Maggiore (CARM, in the proximity of Carmine, Italy), and a more contaminated site closer to Pallanza Bay (LM, in the proximity of Verbania, Italy). The two sediments were chosen because they appeared to have similar sorption properties (Section 6.2.7, Table 6.1) but differed in level of DDX contamination ( $\mathrm{DDDX}$ was $\sim 10$ fold higher in LM than CARM, data not shown) as well as redox properties (LM sediment was black with the exception of a thin light brown layer at the top, whereas CARM sediment was light brown throughout, suggesting that the conditions in LM were more reducing).

\subsubsection{Field deployment of PE.}

For field deployment, the PRC-loaded PE was carried to ENI Donegani Institute (Novara, Italy) where it was stored at room temperature in foil packets for 1 mo. The PE was then mounted in metal frames and deployed at the sediment-water interface in Lake Maggiore in June of 2013. The sampler extended $40 \mathrm{~cm}$ into sediment bed and $20 \mathrm{~cm}$ above the sediment water interface. Sediment from the near vicinity of the sampler (F1) and $\sim 10 \mathrm{~m}$ away from the sampler (F2) were also collected via a grab sampler. The sediment samples were stored in amber foillined jars at $4{ }^{\circ} \mathrm{C}$, until used either for sediment analysis or ex situ incubations with PE (see below). One set of in situ samplers was retrieved after 10 days and another after 30 days. After

retrieval, the PE was cleaned with a Kimwipe, cut from the frame and stored in foil packets at 4 ${ }^{\circ} \mathrm{C}$ for $\sim 2$ months until sub-sectioned into $10 \mathrm{~cm}$ slices, and extracted with solvent.

\subsubsection{Jar incubations.}

We performed jar incubations of PE to determine if the behavior of the PRC and target compounds matches what we observed in the field-deployed PE. Pre-homogenized F2 sediment 
was placed into three $500 \mathrm{~mL}$ jars, and two pieces of PE (2.5 by $2.5 \mathrm{~cm}$ and $25.4 \mu \mathrm{m}$ thickness) were added to each jar (total of 6 PE strips). In each jar, the two PE strips were placed horizontally and parallel to each other, with at least $3 \mathrm{~cm}$ of sediment between each other and the top or bottom of the jar. PE strips were removed after 5, 10 (duplicate), 20 and 30 (duplicate) days (total of 6 PE pieces). The experiment was repeated with two other sediments (CARM and LM), which had been collected from Lake Maggiore during a previous field campaign (Section 6.2.2), and stored in the freezer until use. Because of the smaller amount of sediment available, the jar incubations with CARM and LM sediment were conducted in $125 \mathrm{~mL}$ amber jars, with one PE piece per jar, but still maintaining at least $3 \mathrm{~cm}$ of sediment around the PE to avoid edge effects.

\subsubsection{Tumbling experiments.}

To determine the equilibrium concentration of target analytes in the three sediments ( $F 2$, CARM, LM), as well as to be able to infer the apparent sediment-water partition coefficient for DDE and DDD, a last set of PE/sediment incubations was performed under continuously mixed conditions. The tumbling setup was adapted from Lohmann et al. ${ }^{23}$ In short, a ground glass stoppered, round bottom flask was charged with 30-40 g of wet sediment ( 20 g dry sediment), 100 mL Milli-Q water, and 50 mg PRC-loaded $25 \mu \mathrm{m}$-thick PE. Each tumbling experiment was performed in duplicate. The flasks were placed at room temperature, inside a light-excluding cardboard drum on a roller table. After 1 month, the PE was removed from each flask, wiped with a Kim-wipe, extracted with solvent and analyzed. An aliquot of the sediment slurry from each experiment (except for the spiked autoclaved sediment) was dried overnight in a $60{ }^{\circ} \mathrm{C}$ oven, and saved for chemical analysis.

Suspecting that DDT was susceptible to degradation in the sediments used, two additional set of tumbling experiments were performed with F2 sediment spiked with a mixture of $\mathrm{d}_{8} 4,4^{\prime}$-DDT and ${ }^{13} \mathrm{C}$ PCB 153. First, two flasks containing autoclaved F2 sediment and PE in the same ratio as above, were tumbled for 1 month. Second, a set of flasks containing the spiked F2 sediment, was tumbled in similar conditions for 4-60 d. The concentrations the spiked chemicals were quantified in PE and the sediments (SI-2). 


\subsubsection{PE Extraction.}

The field PE samples were extracted along with trip blanks (used to characterize the concentration of PRCs at time zero), and laboratory blanks. Prior to the first extraction, the PE was spiked with a known amount of a mixture of surrogate standards: ${ }^{13} \mathrm{C}$ PCB 70, $\mathrm{d}_{8} 4,4$ '- DDD, $\mathrm{d}_{8} 2,4$ '-DDT and ${ }^{13} \mathrm{C}$ PCB 170 . Three $20 \mathrm{~mL}$ DCM extractions for each $50 \mathrm{mg}$ piece of PE were combined in a round bottom flask, and concentrated using rotary evaporation. The extract volume was further concentrated to $\sim 100 \mu \mathrm{L}$ and spiked with a known amount of injection compounds ${ }^{13} \mathrm{C}$ PCB 97 and ${ }^{13} \mathrm{C}$ PCB 167. The laboratory PE exposed samples (tumbling and jar), were extracted in a similar fashion, but with a slightly different combination of recovery and injection compounds.

\subsubsection{Sediment Characterization.}

For each sediment (F2, CARM, LM) used in laboratory experiments, we characterized the water content, the fractions of organic carbon $\left(f_{O C}\right)$ and black carbon $\left(f_{B C}\right)$, and the bulk concentration of DDXs. The $\mathrm{f}_{\mathrm{OC}}$ and $\mathrm{f}_{\mathrm{BC}}$ (CTO-375) were measured according to standard procedures, ${ }^{24}$ noting that the sediment used for $\mathrm{f}_{\mathrm{BC}}$ determination was combusted at $375{ }^{\circ} \mathrm{C}$ directly in the silver capsule used for eventual CHN analysis, instead of in a ceramic crucible with subsequent transfer to a silver capsule. The concentrations of DDXs in bulk sediment were determined from $1 \mathrm{~g}$ aliquots of oven dried $\left(60^{\circ} \mathrm{C}, 24 \mathrm{~h}\right)$ and ground sediment which were loaded into metal extraction cells, spiked with the same surrogate standards as the field PE samples, and extracted using an accelerated solvent extractor (Dionex ASE 200, Sunnyvale, CA), with a mixture of 90:10 DCM:methanol, at $100^{\circ} \mathrm{C}$ and 1000 psi. Activated copper (Granular Copper, 20-30 mesh, JT Baker, treated with $1 \mathrm{~N}$ nitric acid) was added to the solvent extract for removal of elemental sulfur. The solvent extracts were then concentrated under a gentle stream of nitrogen to a final volume of $\sim 1 \mathrm{~mL}$, and spiked with injection compounds prior to GCMS analysis.

\subsubsection{Chemical Analysis.}

Quantifications of DDXs in the PE and sediment samples (with the exception of spiked sediments, see SI-2), were done on an Agilent 6890 GC coupled with a mass spectrometer (JEOL GCmate, JOEL Ltd, Tokyo, Japan). The parameters of the analysis have been summarized elsewhere. ${ }^{25}$ We mention here that employing $\mathrm{d}_{8} 2,4$ '-DDT as a recovery standard, 
without any of its potential degradation products ( $\mathrm{d}_{8} 2,4^{\prime}$-DDE or $\mathrm{d}_{8} 2,4^{\prime}$-DDD) added to the sample, ensured that the amount of instrument-related DDT degradation could always be evaluated in each sample (as opposed to relying on detecting the presence of degradation in standard solution containing only DDT). Typically, if samples showed any measurable DDT degradation (seen as appearance of a $\mathrm{d}_{8} 2,4^{\prime}$-DDD peak), the guard column was cut and the analysis was repeated

Table 6.1 $\log K_{d}$ values determined from PE/sediment tumbling experiments $\left(K_{d, T}{ }^{a}\right)$, and from fits of diffusion model to PRC loss from field deployed $\left(K_{d, F}^{b}\right)$ and PRC loss from laboratory incubated PE $\left(K_{d, L}^{c}\right)$ in various sediments collected from Lake Maggiore.

\begin{tabular}{|c|c|c|c|c|c|c|c|c|}
\hline \multirow[t]{2}{*}{ Sediment } & F1 & \multicolumn{3}{|c|}{ F2 } & \multicolumn{2}{|c|}{ CARM } & \multicolumn{2}{|c|}{ LM } \\
\hline & $\log K_{d, T}$ & $\log K_{d, T}$ & $\log K_{d, F}$ & $\log K_{d, L}$ & $\begin{array}{l}\log \\
K_{d, T}\end{array}$ & $\log K_{d, L}$ & $\log K_{d, T}$ & $\log K_{d, L}$ \\
\hline 2,4'-DDE & n.d. ${ }^{\mathrm{d}}$ & 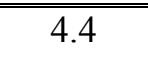 & & & 3.9 & & 3.9 & \\
\hline $4,4^{\prime}$-DDE & 4.3 & 4.3 & 4.3 & $3.8 \pm 0.3$ & 3.9 & $3.6^{\mathrm{e}}$ & 3.9 & $3.6^{\mathrm{f}}$ \\
\hline $2,4^{\prime}-\mathrm{DDD}$ & 3.8 & 3.9 & & & 3.7 & & 3.6 & \\
\hline $4,4^{\prime}$-DDD & 4.0 & 4.1 & 3.8 & $3.8 \pm 0.3$ & 3.8 & $3.6 \pm 0.7$ & 3.8 & $3.3 \pm 0.2$ \\
\hline$f_{\mathrm{OC}}(\%)$ & $2.42 \pm 0.16$ & & $2.23 \pm 0.10$ & & & 0.02 & $1.26=$ & 0.03 \\
\hline$f_{B C}(\%)$ & $0.35 \pm 0.01$ & & $0.33 \pm 0.04$ & & & $=0.03$ & $0.17=$ & 0.03 \\
\hline
\end{tabular}

${ }^{\text {a }} K_{d, T}=C_{S E D} / C_{P E}{ }^{*} K_{P E W}$, with $K_{P E W}$ values from Hale et al. ${ }^{26}$. Error in $\mathrm{K}_{\mathrm{d}}$ values calculated from duplicate experiments was $<0.05 \log$ units. Even when considering heterogeneity in sediment concentration from triplicate $\mathrm{C}_{\mathrm{SED}}$ measurements, error in $\mathrm{K}_{\mathrm{d}}$ was still less than 0.1 log units across all sediments.

${ }^{\mathrm{b}}$ Errors of fitted coefficient could not be calculated because field data only contained two timepoints.

${ }^{c}$ Error bars on fitted $K_{d}$ value were calculated based on a $95 \%$ confidence interval.

${ }^{\mathrm{d}}$ Not determined because 2,4'-DDE concentration in sediment was below detection.

${ }^{\mathrm{e}}$ Only upper limit of $95 \% \mathrm{CI}\left(\mathrm{K}_{\mathrm{d}}<10^{4}\right)$ could be calculated due to small PRC loss $<20 \%$.

${ }^{\text {f }}$ Only upper limit of $95 \% \mathrm{CI}\left(\mathrm{K}_{\mathrm{d}}<10^{4}\right)$ could be calculated due to small PRC loss $<20 \%$.

\subsection{Results and Discussion}

\subsubsection{PRC loading and detection limits.}

The PRC concentrations ranged from 100 to $400 \mathrm{ng} / \mathrm{g}$ PE and were more than a factor of 100 higher than the limits of detection (Table S1). The variability in the loaded PE $(N=5)$, was less 
than $6 \%$ for all the PRCs in the field deployed PE, and less than $10 \%$ for most PRCs in the laboratory PE, with the exception of ${ }^{13} \mathrm{C}$ PCB $28(13 \%),{ }^{13} \mathrm{C}$ PCB $111(11 \%)$ and ${ }^{13} \mathrm{C}$ PCB 153 $(15 \%)$.

\subsubsection{Fractional loss of PRCs after field deployment.}

The measured PRC losses after 10 and 30 days deployment of PE into the sediment bed at the field site suggested that the releases of the PCB PRCs, as well as those of ${ }^{13} \mathrm{C} 4,4$ '-DDE and ${ }^{13} \mathrm{C} 4,4$ '-DDD, were all diffusion mediated. The fractions of PRC remaining after 10- and 30-day deployments were consistent with diffusion profiles (Figure 6.1) obtained using the model of Fernandez et al. (2009) ${ }^{14}$ and using $K_{\mathrm{d}}$ values $\left(\mathrm{L}_{\mathrm{W}} / \mathrm{kg}_{\mathrm{S}}\right)$ ranging from $10^{3.6}-10^{4.0}$ for DDD and $10^{4.1}-10^{4.5}$ for DDE. Furthermore, the $K_{\mathrm{d}}$ values deduced from the PRC loss in the PE strip deployed at $0-10 \mathrm{~cm}$ into the sediment $\left(10^{4.3}\right.$ for DDE and $10^{4.0}$ for DDD), matched quite well the independently measured $K_{\mathrm{d}}$ values from ex situ tumbling experiments with the same sediment (Table 6.1). The measured $K_{d}$ values make sense relative to each other, as $K_{O W}$ of DDE is larger than $\mathrm{K}_{\mathrm{OW}}$ of DDD ( $10^{6.5}$ and $10^{6.0}$, respectively). Furthermore, across the four depth horizons (0$10,10-20,20-30$ and $30-40 \mathrm{~cm}$ ), the PRC loss varied at most by $10 \%$, suggesting that $\mathrm{K}_{\mathrm{d}}$ does not significantly vary with depth at this site (Figure 6.1 and Gschwend et al. ${ }^{17}$ ).
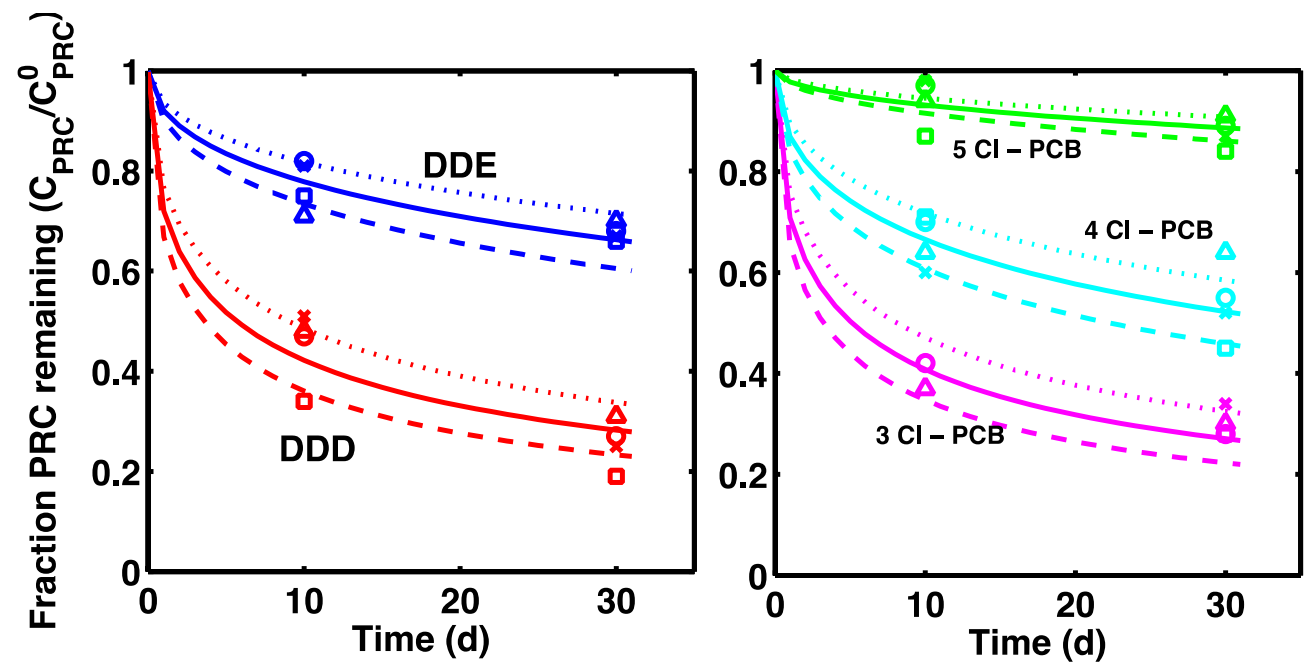

Figure 6.1 Fractional PRC remaining in PE as a function of time, after deployment in sediment at various depth horizons: 0-10 cm (squares), 10-20 cm (crosses), 20-30 cm (circles), and 30-40 $\mathrm{cm}$ (triangles). Solid lines represent the expected PRC release using the diffusion model of Fernandez et al. ${ }^{14}$, assuming $\mathrm{K}_{\mathrm{d}}\left(\mathrm{L}_{\mathrm{W}} / \mathrm{kg}_{\mathrm{sed}}\right)$ values of $10^{3.8}\left({ }^{13} \mathrm{C} 4,4^{\prime}\right.$-DDD $), 10^{4.3}\left({ }^{13} \mathrm{C} 4,4^{\prime}\right.$-DDE), $10^{4.5}\left({ }^{13} \mathrm{C}\right.$ PCB 28), $10^{4.4}\left({ }^{13} \mathrm{C}\right.$ PCB 47) and $10^{5.5}\left({ }^{13} \mathrm{C}-\mathrm{PCB} 111\right)$. Dotted and dashed lines 
represent the results of the same diffusion model, using $K_{d}$ values $0.2 \log$ units lower and higher, respectively. The measured losses of each PRC compound are consistent with diffusion-driven transport.

\subsubsection{PRC loss and target uptake in static ex-situ incubations.}

For DDE/DDD and PCB PRCs, the losses of PRCs measured in laboratory incubations were similar to that observed in the field PE. For example, while there was some difference $(\sim 10 \%)$ in the amount of PRC loss measured in field PE versus the PE incubated in F2 sediment (collected from the vicinity of samplers), the overall shape of loss curve was similarly well captured by the diffusion model ${ }^{14}$ (Figure 6.2). This was also the case for the PE incubated in two additional sediments, CARM and LM (Figure 6.2).

The transfer between PE and sediment of PRC and target analytes was isotropic for both DDE and DDD in all three jar incubations (Figure 6.2), as expected for diffusion-mediated transport. The sum of observed PRC loss and corresponding target accumulation was $1 \pm 0.1$ across all sampled time points (e.g. $1.02 \pm 0.03$ for DDE, and $0.98 \pm 0.08$ for DDD for PE in F2). However, the $K_{d}$ values that fit the measured PRC loss, transformed throughout this manuscript to units of $\mathrm{L}_{\mathrm{W}} / \mathrm{kg}_{\text {sed }}$ (Figure 6.2) were smaller on average, by 0.3 log units (range 0.2 to 0.5 ) than the measured $K_{d}$ values (also in $\mathrm{L}_{\mathrm{W}} / \mathrm{kg}_{\mathrm{sed}}$ ) from tumbling experiments (Table 6.1). Hence, though the diffusion model ${ }^{14}$ accurately captures both the release and uptake of DDE and DDD, the model-derived $K_{d}$ is a fitted value, which will only equal the true $K_{d}$ value when all the assumptions of the diffusion model are met. First, bioturbation or groundwater flow could violate the static sediment bed assumption, leading to increased PRC loss and larger $K_{d}$ values in the field compared to laboratory incubation done with the same sediment (e.g. $\mathrm{K}_{\mathrm{d}, \mathrm{DDE}}$ from PRC loss in field PE $10^{4.3}$ vs. lab F2 PE $10^{3.8}$ ). Second, local disequilibrium effects could lead to PRCbased $K_{d}$ values that are lower than the tumbling-based $K_{d}$ (e.g, for DDE in CARM and LM, tumbling- $\mathrm{K}_{\mathrm{d}} 10^{3.9}$ vs. PRC- $\mathrm{K}_{\mathrm{d}} 10^{3.6}$ ). At the same time, it should be noted that uncertainties associated with $\mathrm{K}_{\mathrm{d}}$ values estimated using organic and black carbon measurements can easily exceed $0.5 \log$ units as well; $K_{O C}$ alone can vary by one order of magnitude depending on organic carbon type. ${ }^{27}$ 

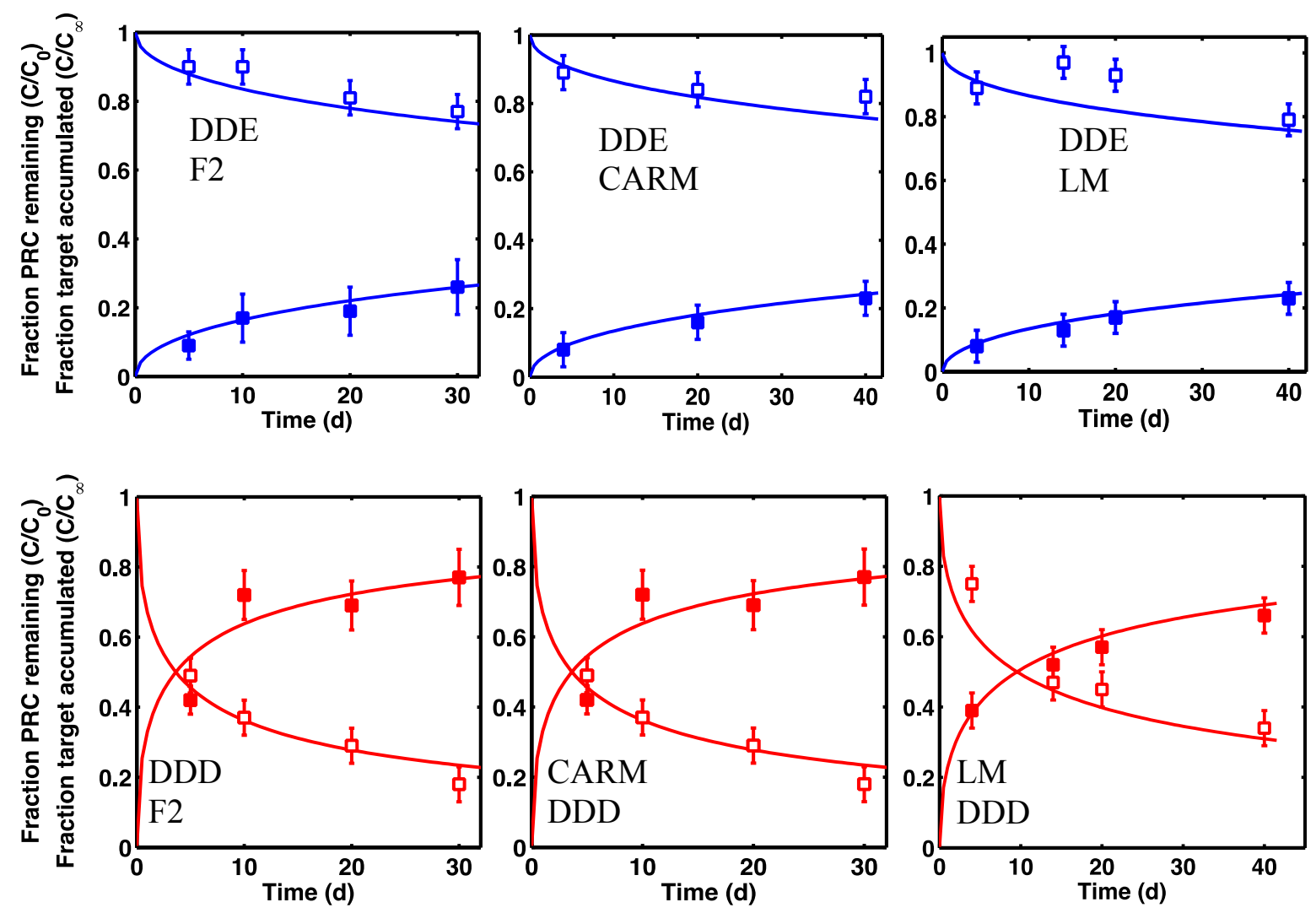

Figure 6.2 Fraction of PRC remaining (filled symbols) and fraction of target compound accumulated (empty symbols) for static incubation of PE in F2, CARM and LM sediment for 4,4'-DDE (top panels) and 4,4'-DDD (bottom panels). Fractional accumulation calculated as the ratio of concentration of target analyte measured in PE from static incubation divided by the concentration of the same analyte in tumbled PE. Lines represent the diffusion model of Fernandez et al. ${ }^{14}$ with $\mathrm{K}_{\mathrm{d}}\left(\mathrm{L}_{\mathrm{W}} / \mathrm{kg}_{\mathrm{SED}}\right)$ for DDE of $10^{3.8}(\mathrm{~F} 2), 10^{3.6}(\mathrm{CARM}), 10^{3.6}(\mathrm{LM})$ and for DDD of $10^{3.8}$ (F2), $10^{3.6}$ (CARM), $10^{3.3}$ (LM). Error bars for fraction of PRC remaining calculated based on one SD of PRC concentration in $\mathrm{T}=0 \mathrm{PE}$, and for fraction of target accumulation based on one SD of instrument error.

Thus, though potentially susceptible to chemical transformations in the sediment, the exchange of DDE and DDD between passive samplers and sediments beds appears to be mainly diffusion-mediated. The reaction rates associated with potential transformations of DDE and DDD in both field and laboratory conditions were likely slower compared to the rates of diffusion into the sediment bed. PE/sediment exchange timescales were on the order of days (70 days for DDE and 15 days for DDD) while previous measurements of degradation rates imply half-lives on the order of years (3-30 $\mathrm{yr}$ for DDE ${ }^{11}$ and $10 \mathrm{yr}$ for DDD in sediment beds ${ }^{28}$ ). This is further supported by the fact that in the 30 day $\mathrm{PE} /$ sediment tumbling experiments, no loss of 
DDE or DDD was observed in the sediment comparing concentrations found pre and post tumbling (data not shown).

\subsubsection{Reaction-driven DDT PRC loss.}

In contrast, the loss of DDT PRC measured in the field deployed PE (Figure 6.3), and in the laboratory incubations (Figure 6.4) was not consistent with diffusion-mediated transport. The loss of DDT PRC did not slow down with time, as it would be the case for a diffusion curve (Figure 6.3, left). Furthermore, since $K_{d}$ is usually correlated with $K_{O W}$, and DDE and DDT have similar $K_{O W}$ values $\left(10^{6.5}\right.$ and $10^{6.8}$, respectively $\left.{ }^{29}\right)$ we would expect both compounds to have similar $K_{d}$ values and implicitly, similar PRC losses.
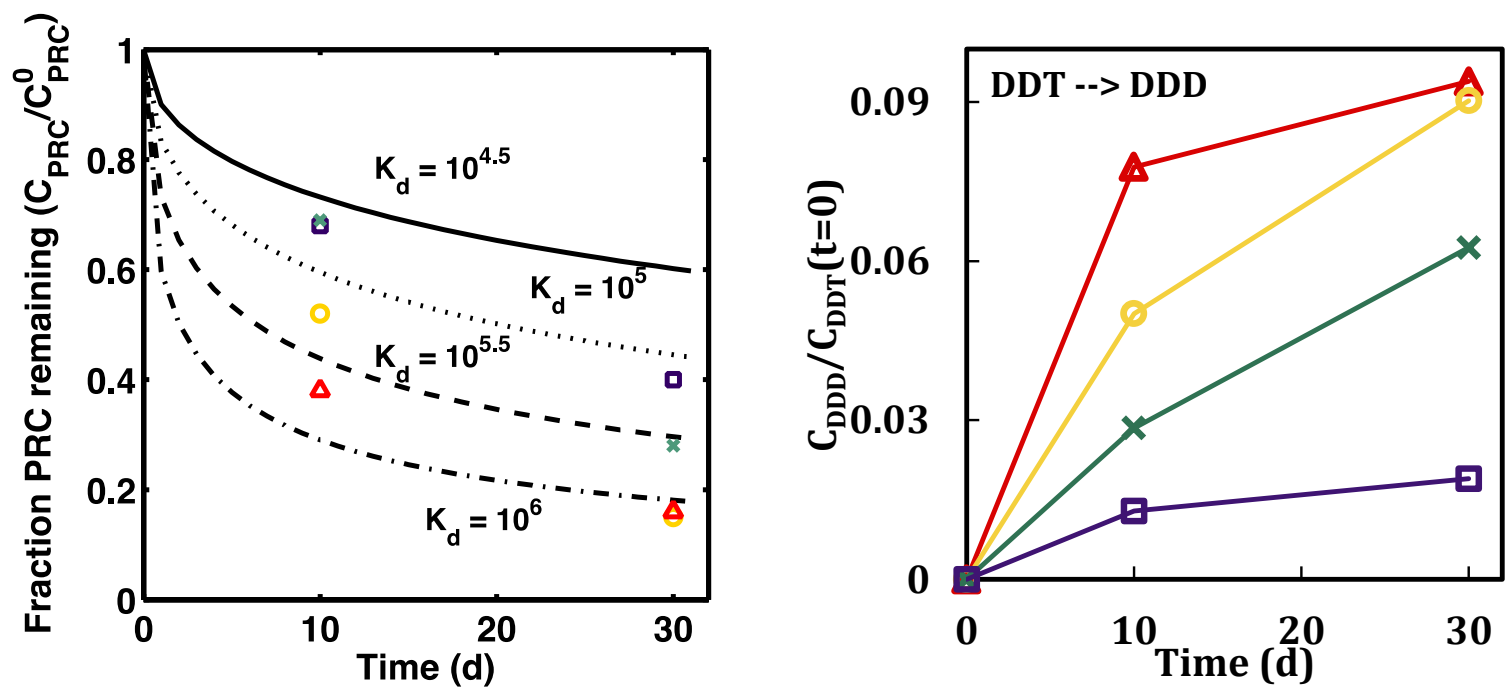

Figure 6.3 Fraction of ${ }^{13} \mathrm{C} 2,4^{\prime}$-DDT PRC remaining in PE (left), and measured in PE as the degradation product ${ }^{13} \mathrm{C} 2,4^{\prime}$-DDD (right), after deployment in sediment at various depth horizons: 0-10 cm (squares), 10-20 cm (crosses), 20-30 cm (circles), and 30-40 cm (triangles). Lines in left figure represent the expected PRC release using the diffusion model of Fernandez et al. ${ }^{14}$, assuming $\mathrm{K}_{\mathrm{d}}\left(\mathrm{L}_{\mathrm{W}} / \mathrm{kg}_{\mathrm{sed}}\right.$ ) values of $10^{4.5}$ (solid), $10^{5.0}$ (dot), $10^{5.5}$ (dash), and $10^{6}$ (dot-dash). As opposed to PRCs in Figure 1, the measured DDT PRC loss varies significantly with depth. In addition, the PRC loss from 10-20 and 20-30 cm depth horizons is not consistent with the shape of a diffusion profile. For the $0-10 \mathrm{~cm}$ and $30-40 \mathrm{~cm}$, the PRC loss appears to be loosely consistent with diffusion into a sediment with a $\mathrm{K}_{\mathrm{d}}$ value of $10^{5}$ and $10^{6}$, respectively. Lines in right figure are drawn to guide the eye and are not model fits.

In contrast, the DDT PRC was lost to a substantially greater extent than the DDE PRC (at least by a factor of 2 after 30 days). Also, the $\mathrm{K}_{\mathrm{D}}$ values $\left(10^{5}-10^{6}\right)$ required to fit the DDT-PRC 
losses are 1-2 orders magnitude larger than either the model fit or measured $K_{d}$ values of DDE $\left(10^{4.3}\right.$, Table 6.1). Lastly, the amount of DDT-PRC recovered in the PE was found to decrease significantly with depth, more than it could be explained by loading variability $(\sim 2 \%)$, and inconsistent with the behavior of all other PRCs, whose loss varied little with depth (Figure 6.1).

Similar to the field deployment case, the losses of the DDT PRC measured in PE strips incubated in sediments in the laboratory, were also not consistent with diffusive transport. While the loss of the DDE PRC (similar size and $K_{O W}$ to DDT, as mentioned above) varied at most by $10 \%$ across the PEs incubated in three different sediments (e.g. 8 - 18\% lost after 20 days, Figure 6.2), the DDT PRC loss differed greatly (e.g. after 20 days lost 43\% in CARM-PE, and more than $90 \%$ in LM-PE). The variable loss of DDT PRC across the different sediment incubations supports the hypothesis that reactive processes occurring in the sediment, as opposed to inside the PE, are accelerating the PRC loss.

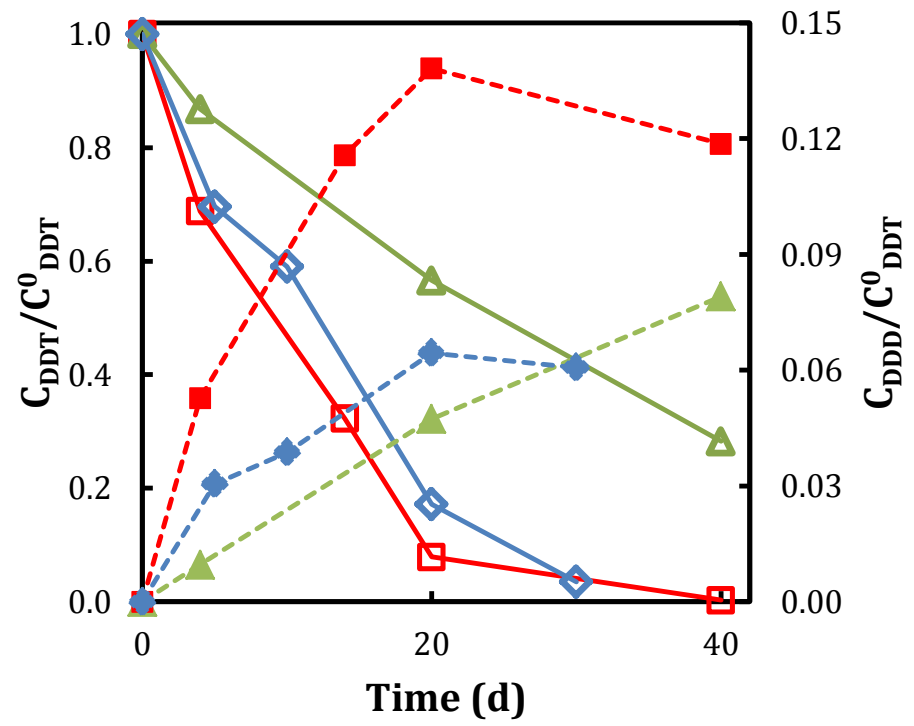

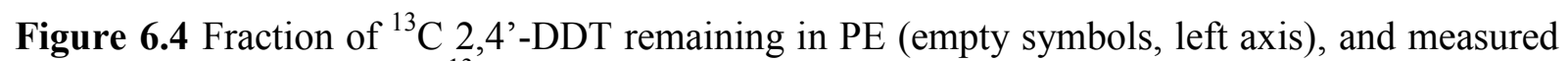
as its degradation product ${ }^{13} \mathrm{C} 2,4^{\prime}$-DDD (filled symbols, right axis) as a function of time after incubation in CARM (green triangles), LM (red squares) and F2 (blue diamonds). Lines are drawn to guide the eye and are not model fits. 


\subsubsection{Accumulation of PRC degradation products in the PE.}

The fact that the loss of the ${ }^{13} \mathrm{C} 2,4$ '-DDT PRC was driven by reactions is confirmed by the corresponding accumulation in the $\mathrm{PE}$ of its degradation product, ${ }^{13} \mathrm{C} 2,4$ '-DDD. This labeled degradate's concentration in PE increased with depth into the sediment (Figure 6.3). The amount of degradation product was on the order of $2-20 \mathrm{ng} / \mathrm{g}$ PE, at least 10 times more than the limit of detection (Table S1) and its identity was confirmed by its retention time and the observed ratio of the confirming and quantitation ion intensities. We are confident that the amount of degradation product measured came from reactions occurring in the environment, and not degradation during the PE extraction or GC-MS analysis. The degradation of $\mathrm{d}_{8} 2,4$ '-DDT recovery standard, added to PE prior to extraction, was closely monitored and analysis conditions were carefully maintained such that $\mathrm{d}_{8}$ 2,4'-DDD was always below detection. Accumulation of the PRC degradation product ${ }^{13} \mathrm{C} 2,4$ '-DDD was also observed in the laboratory incubations, and the measured concentration of ${ }^{13} \mathrm{C} 2,4^{\prime}$-DDD in PE increased with time and with the amount of observed DDT PRC loss (Figure 6.4). In both field and laboratory incubations, up to $10 \%$ of the initial DDT PRC concentration was recovered as ${ }^{13} \mathrm{C} 2,4^{\prime}$-DDD in the PE (Figure 6.3, Figure $6.4)$.

\subsubsection{Reaction diffusion model.}

Lastly, we evaluated whether a reaction diffusion model could fit the behavior of the DDTPRC (Equation 3 inverted with Matlab). Since the observed PRC loss is due to both diffusion of the PRC into the sediment bed and reaction in the sediment, the reaction rate can only be fit for a given $K_{d}$ (which affects $K_{P E S E D}$ and $\Psi$ in Equation 3). Applying the reaction diffusion model to the observed PRC loss from the field deployed PE, and assuming the same value of $K_{d}$ of DDT as the model derived (Figure 6.1) $K_{d}$ value of DDE (similar $K_{O W}$ values of DDE and DDT, see Section 6.3.4), we obtain fitted reaction rates that increase with depth into sediment bed: $0.09 \mathrm{~d}^{-1}$ at $0-10 \mathrm{~cm}$ to $0.9 \mathrm{~d}^{-1}$ at $30-40 \mathrm{~cm}$ (Figure 6.5). The fit of PRC loss over time is greatly improved over using the diffusion-only model (Figure 6.5 vs. Figure 6.3 left). Previous studies found that DDT degradation in sediment slurries depended on oxidation reduction potential $\left(\mathrm{k}_{\mathrm{r}}\right.$ ranged from 0.03 to $0.3 \mathrm{~d}^{-1}$ for $E_{h}$ values of -150 to $450 \mathrm{mV}$ ), and that DDD was the major degradation product in strongly reducing conditions. ${ }^{7}$ Thus, the magnitude and increase with depth of the fitted reaction rate make sense given that (1) the oxidation reduction potential is likely to become 
more reducing with depth into sediment bed and (2) the amount of ${ }^{13} \mathrm{C} 2,4$ '-DDD degradation product measured in the PE also increases with depth (Figure 6.3 right).

Fits of PRC losses observed in laboratory incubations of PE in the sediments yielded reaction rates that were larger than for the field PE, ranging from $0.5 \mathrm{~d}^{-1}$ (CARM) to $3.5 \mathrm{~d}^{-1}$ (LM). As was the case for modeling the results of the field deployments, the fits to the DDT PRC improved over using a diffusion-only model (compare Figure 6.4 with Figure 6.6). The fitted reaction rate deduced from the PRC loss in F2 PE is almost an order of magnitude larger, due to larger PRC loss observed in the static laboratory incubation. The difference in reaction rates could be a result of the assumptions made in the model (e.g. lower $\mathrm{K}_{\mathrm{d}}$ values needed to explain PRC loss from laboratory incubated PE versus field deployed, see captions of Figure 6.1 and Figure 6.2, and Table 6.1), or may actually be reflecting more favorable conditions for degradation of DDT in the laboratory, due to higher temperatures or changes in redox potential of the sediment after removal from the field.

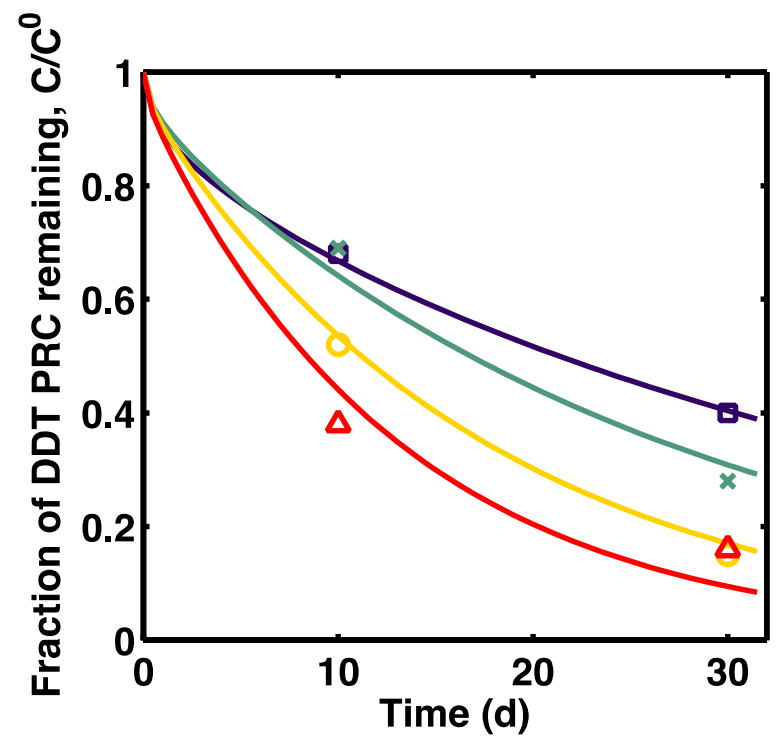

Figure 6.5 Fraction of DDT PRC remaining after 10 and 30 day deployments at various depths into the sediment $0-10 \mathrm{~cm}$ (squares), $10-20 \mathrm{~cm}$ (crosses), 20-30 cm (circles), and 30-40 cm (triangles). Lines represent reaction diffusion model results using $\mathrm{K}_{\mathrm{d}}$ for DDT equal to $K_{d}$ of DDE deduced from PRC loss in the sediment, and reaction rates for DDT degradation from top to bottom of graph of 0.1 (blue), 0.3 (green), 0.7 (yellow) and $1 \mathrm{~d}^{-1}$ (red). 
Instability of DDT in F2 sediment on timescales of days-weeks was also confirmed by the poor recovery of a labeled DDT spike in tumbling experiments with F2 sediment (only 1-18\% of initial $\mathrm{d}_{8} 4,4^{\prime}$-DDT spike recovered, versus $91 \pm 12 \%$ of spiked ${ }^{13} \mathrm{C}$ PCB 153$)$. Even when the sediment was autoclaved prior to incubation, no labeled DDT was measurable in PE tumbled with the sediment that was also spiked, suggesting that the degradation may be abiotic. The lack of native DDT in the field-collected F1 and F2 sediments in fact further supports the hypothesis of DDT reactivity. For an average sedimentation rate of $0.5 \mathrm{~cm} /$ year, ${ }^{30}$ the average age of the upper $10 \mathrm{~cm}$ would be about 10 years ( 0 at the top and 20 years at $10 \mathrm{~cm}$ ), implying that DDT reacts on timescales shorter than 10 years.

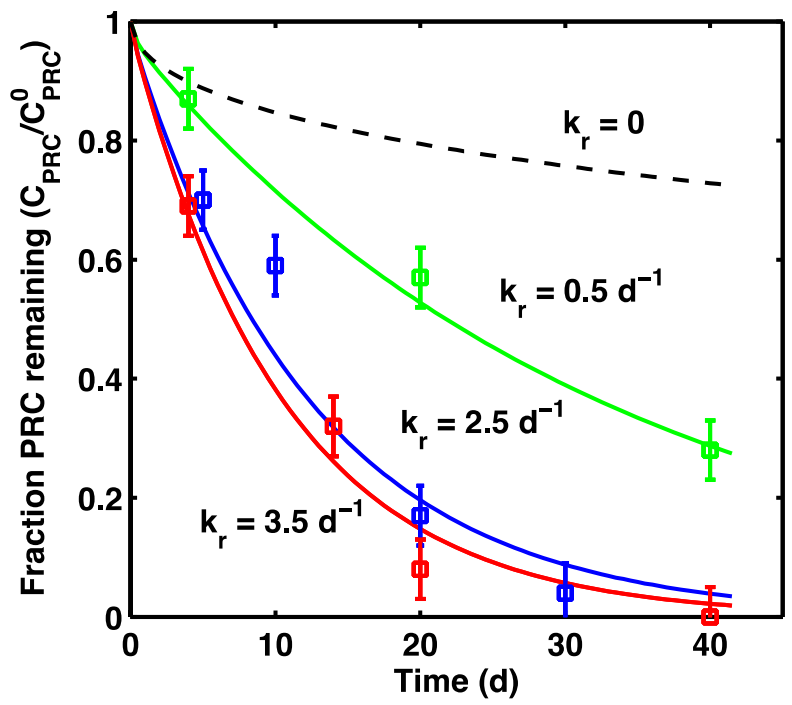

Figure 6.6 Model fits (solid lines) for the fraction of ${ }^{13} \mathrm{C} 2,4$ '-DDT remaining in PE as a function of time after incubation in CARM (green triangles), LM (red squares) and F2 (blue diamonds). Reaction rates ranged from $0.5 \mathrm{~d}^{-1}$ to $3.5 \mathrm{~d}^{-1}$. Also shown for comparison, the model prediction with no reaction (dashed line).

Altogether, the reactivity of DDT in the sediments was confirmed by (1) accelerated loss over other PRCs with similar transport properties $\left(D_{W}\right.$ and $\left.K_{O W}\right),(2)$ accumulation of the PRC degradation product, ${ }^{13} \mathrm{C} 2,4^{\prime}$-DDD (3) larger accumulation of ${ }^{13} \mathrm{C} 2,4$ '-DDD with increasing reducing conditions and (4) instability of DDT in a sediment slurry. The range of fitted reaction rates from both field and laboratory $\operatorname{PE}\left(0.09 \mathrm{~d}^{-1}\right.$ to $\left.3.5 \mathrm{~d}^{-1}\right)$ is in line with degradation rates previously measured in anaerobic sediment slurries, ${ }^{7,8}$ or in aqueous systems amended with zero valent iron. ${ }^{31}$ However, the best fit reaction rates were fast compared to other studies which 
report much slower degradation rates $\left(0.08-2 \mathrm{yr}^{-1}\right) \cdot{ }^{9,28}$ Although it remains to be confirmed that the magnitudes of the reaction rate derived using the reaction-diffusion model are accurate, the results so far suggest that passive sampling is a promising tool for measuring in situ reactivity of compounds in sediments.

Although the accumulation of the ${ }^{13} \mathrm{C} 2,4$ '-DDD degradation product of the DDT-PRC supports the hypothesis that the DDT-PRC is undergoing degradation, the amount of ${ }^{13} \mathrm{C} 2,4^{\text {' }}$ DDD that is recovered in the PE is at most $10 \%$ of the initial DDT-PRC loaded in the PE (Figures 6.3 and 6.4), which is not consistent with DDD being the only degradation product of DDT in the sediment. Based on the reaction diffusion model developed in this paper, the DDTPRC diffuses out into the sediment for about $0.3 \mathrm{~cm}$ (or about two PE half thicknesses). If the DDD were the only degradation product, then the ${ }^{13} \mathrm{C} 2,4$ '-DDD produced would at the same time diffuse further away into the sediment and also back into the PE. However given diffusivity of DDD in the sediment (calculated to be $\sim 10^{-10} \mathrm{~cm}^{2} / \mathrm{s}$ ), the DDD produced would only diffuse $0.01 \mathrm{~cm}$ in the sediment in $1 \mathrm{mo}$. On the other hand, considering the partition coefficients between PE and water $\left(K_{P E W}\right.$ for DDD $\left.10^{5}\right)$ and the sediment water partition coefficients measured for the lake sediments ( $K_{d}$ for DDD of $10^{3.3}$ and $10^{3.8}$, Table 6.1), this would imply that, the majority of the ${ }^{13} \mathrm{C} 2,4^{\prime}$-DDD produced should be in the PE at equilibrium $\left(K_{P E S E D}>\right.$ 10). In turn, this would suggest that the amount of DDT-PRC lost should have been approximately equal to the amount of ${ }^{13} \mathrm{C} 2,4^{\prime}$-DDD accumulated in the PE. Even if the ${ }^{13} \mathrm{C} 2,4^{\prime}-$ DDD were to not reach equilibrium (i.e., behave like the ${ }^{13} \mathrm{C} 4,4^{\prime}$-DDD PRC, which only reaches $\sim 80 \%$ equilibration in 30 days, Figures 6.1 and 6.2 ), the amount of ${ }^{13} \mathrm{C} 2,4^{\prime}$-DDD which accumulated in the PE is still too low. For example, for the PE deployed in the sediment at 30-40 $\mathrm{cm}$, we measured accumulation of ${ }^{13} \mathrm{C} 2,4^{\prime}$-DDD of $9 \%$ relative to the initial amount of DDTPRC left, but if all the DDT-PRC that reacted in the sediment were to transform to DDD, then we should have measured $\sim 64 \%$. Although possible, it is unlikely that the DDD produced could be undergoing degradation as well, given that behavior of the DDD-PRC and DDD target analytes were both consistent with diffusion driven transport (Figures 6.1 and 6.2).

These calculations suggest however, that the degradation of the DDT in the sediment could lead to the formation of other degradation products besides DDD. DDT is know to degrade to 
DDE, for example; but, following the same reasoning as above, if the DDT-PRC was degrading to DDE then we should have measured an accumulation of 2,4 '-DDE in the PE $(\sim 10-20 \%$ of the initial DDT-PRC concentration, assuming all DDT transforming to DDE). In contrast, in both field deployments and laboratory incubations of PE in sediment, there was no measurable accumulation of ${ }^{13} \mathrm{C} 2,4^{\prime}$-DDE (detection limit of $\sim 1 \%$, Table $\mathrm{S} 1$ ), suggesting that DDE was not the major degradation product. Altogether, it appears that the degradation of DDT in the vicinity of PE may lead to more than the known degradation products DDE and DDD, and this conclusion is supported by other studies of DDT degradation in anaerobic sediment slurries which also observed that the sum of DDE and DDD produced only accounted for $15-33 \%$ of the initial DDT spike. ${ }^{7}$
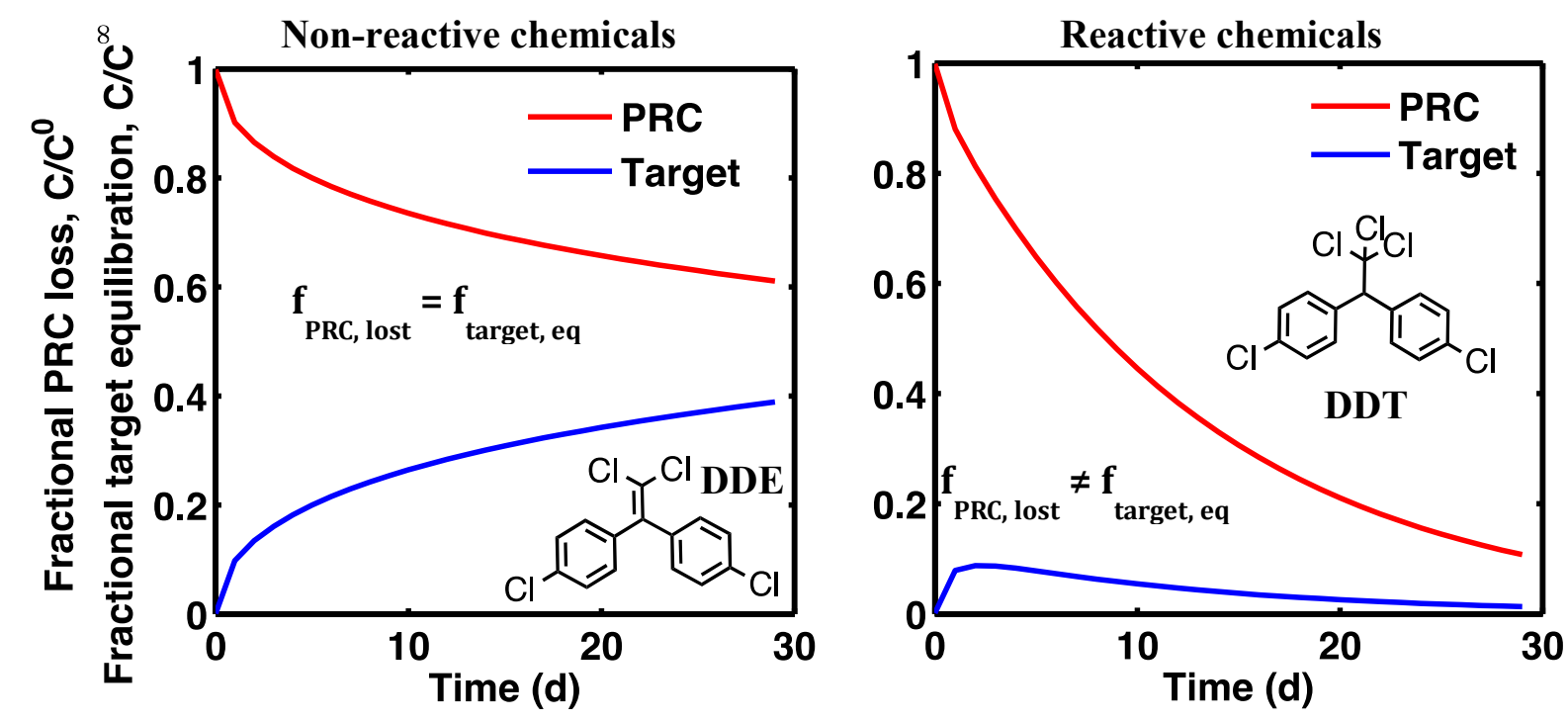

Figure 6.7 Accumulation of target compounds (blue) and release of PRCs (red) for a nonreactive (DDE, left) and for a reactive (DDT, right) compound. Non-reactive herein refers to chemicals whose reaction timescales are much slower than the timescale of $\mathrm{PE} /$ sediment exchange (order of weeks to months for DDXs). Conversely, reactive chemicals have reaction timescales that are shorter or comparable (order of hours to days) to the PE/sediment exchange timescales.

\subsubsection{Implications for interpretation of PRCs and measuring reactive compounds.}

For field deployments, PRCs should be chosen to avoid overlap in isotopic label or isomer state between the DDT PRC and DDE/DDD PRCs (i.e. 2,4'-DDT should be used with 4,4'-DDE 
and DDD, or vice-versa). Using a combination of reactive and non-reactive PRCs can help us understand the processes that are happening in the sediment. If a DDT-PRC is used, and there is no reactivity in the sediment, e.g, the DDT PRC behaves similarly to PCB or DDE PRCs, then the PRC loss can be interpreted similarly to the PRC loss of DDE and DDD. ${ }^{16}$ But if reactivity is present, then we can use the reaction diffusion model to investigate how the accumulation of target compound is affected. Assuming the same reaction rate applies to a target compound, the accumulation of target chemical will be greatly decreased compared to its equilibrium value (Figure 6.7). For example, a reduced uptake of target chemical could be observed when using passive sampling for other organochlorine pesticides which react in the sediments on timescales similar to DDT, such as chlordane or dieldrin. ${ }^{28}$ In addition, if the reactive processes are fast enough to accelerate the PRC loss (Figure 6.1 and Figure 6.5, e.g. diffusion timescales of weeks to months for DDE and DDD, but reaction timescale of DDT of days), then the PRC loss in conjunction with Equation 3 could be used to evaluate in situ degradation rate. This application warrants further investigation because it can greatly enhance our understanding of environmental transformations of chemicals such as organochlorine pesticides but also other chemicals such as pyrethroids, which have been found to undergo reactions on timescales from days to years, depending on chemical structure and on conditions in the sediment. ${ }^{32}$

\section{Acknowledgements}

Funding for this research was provided by ENI S.p.A (through the MIT Energy Initiative, contract numbers 5200003626 and 5210000541). 


\section{References}

1. Mingelgrin, U.; Nasser, A. n., Diagnosis and prognosis of the distribution of contaminants in the geosphere. Viable Methods of Soil and Water Pollution Monitoring, Protection and Remediation 2006, 69, 3-23.

2. Weston, D. P.; Jarman, W. M.; Cabana, G.; Bacon, C. E.; Jacobson, L. A., An evaluation of the success of dredging as remediation at a DDT-contaminated site in San Francisco Bay, California, USA. Environ Toxicol Chem 2002, 21, (10), 2216-2224.

3. US Environmental Protection Agency Overview of Palos Verdes Shelf Superfund Site. http://yosemite.epa.gov/r9/sfund/r9sfdocw.nsf/3dc283e6c5d6056f88257426007417a2/25ce9253a 6ef1bcc88257d4800637d5a!OpenDocument

4. US Environmental Protection Agency Velsicol Chemical Corp. (Michigan) Superfund Site. http://www.epa.gov/region5/cleanup/velsicolmichigan/

5. Lydy, M. J.; Landrum, P. F.; Oen, A. M.; Allinson, M.; Smedes, F.; Harwood, A. D.; Li, H.; Maruya, K. A.; Liu, J., Passive sampling methods for contaminated sediments: state of the science for organic contaminants. Integr Environ Assess Manag 2014, 10, (2), 167-78.

6. Sinkkonen, S.; Paasivirta, J., Degradation half-life times of PCDDs, PCDFs and PCBs for environmental fate modeling. Chemosphere 2000, 40, (9-11), 943-949.

7. Gambrell, R. P.; Reddy, C. N.; Collard, V.; Green, G.; Patrick, W. H., The recovery of DDT, kepone, and permethrin added to soil and sediment suspensions incubated under controlled redox potential and $\mathrm{pH}$ conditions. $J$ Water Pollut Con F 1984, 56, (2), 174-182.

8. Chiu, T. C.; Yen, J. H.; Liu, T. L.; Wang, Y. S., Anaerobic degradation of the organochlorine pesticides DDT and heptachlor in river sediment of Taiwan. B Envion Contam Toxicol 2004, 72, (4), 821-828.

9. Eggen, T.; Majcherczyk, A., Effects of zero-valent iron $(\mathrm{Fe} 0)$ and temperature on the transformation of DDT and its metabolites in lake sediment. Chemosphere 2006, 62, (7), 111625 .

10. Connor, M. S.; Davis, J. A.; Leatherbarrow, J.; Greenfield, B. K.; Gunther, A.; Hardin, D.; Mumley, T.; Oram, J. J.; Werme, C., The slow recovery of San Francisco Bay from the legacy of organochlorine pesticides. Environ Res 2007, 105, (1), 87-100.

11. Eganhouse, R. P.; Pontolillo, J., DDE in sediments of the Palos Verdes Shelf, California: in situ transformation rates and geochemical fate. Environ Sci Technol 2008, 42, (17), 6392-8.

12. Tomaszewski, J. E.; Luthy, R. G., Field deployment of polyethylene devices to measure PCB concentrations in pore water of contaminated sediment. Environ Sci Technol 2008, 42, (16), 6086-91.

13. Apell, J. N.; Gschwend, P. M., Validating the use of performance reference compounds in passive samplers to assess porewater concentrations in sediment beds. Environ Sci Technol 2014, 48, (17), 10301-7.

14. Fernandez, L. A.; Harvey, C. F.; Gschwend, P. M., Using performance reference compounds in polyethylene passive samplers to deduce sediment porewater concentrations for numerous target chemicals. Environ Sci Technol 2009, 43, (23), 8888-94.

15. Bao, L. J.; Jia, F.; Crago, J.; Zeng, E. Y.; Schlenk, D.; Gan, J., Assessing bioavailability of DDT and metabolites in marine sediments using solid-phase microextraction with performance reference compounds. Environ Toxicol Chem 2013, 32, (9), 1946-1953.

16. Liu, H. H.; Bao, L. J.; Zhang, K.; Xu, S. P.; Wu, F. C.; Zeng, E. Y., Novel passive sampling device for measuring sediment-water diffusion fluxes of hydrophobic organic chemicals. Environ Sci Technol 2013, 47, (17), 9866-73. 
17. Fernandez, L. A.; Lao, W.; Maruya, K. A.; White, C.; Burgess, R. M., Passive sampling to measure baseline dissolved persistent organic pollutant concentrations in the water column of the Palos Verdes Shelf Superfund site. Environ Sci Technol 2012, 46, (21), 11937-47.

18. Gschwend, P. M., Validation of the polyethylene passive in situ sampling protocol: assessment of DDT and its degradates in the sediments of Lago Maggiore, Italy. In 2014 (in prep).

19. Hollenbeck, K. J. INVLAP.M: A matlab function for numerical inversion of Laplace transforms by the de Hoog algorithm. http://www.isva.dtu.dk/staff/karl/invlap.htm

20. Thompson, J. M.; Hsieh, C. H.; Luthy, R. G., Modeling uptake of hydrophobic organic contaminants into polyethylene passive samplers. Environ Sci Technol 2015, 49, (4), 2270-7.

21. Booij, K.; Smedes, F.; van Weerlee, E. M., Spiking of performance reference compounds in low density polyethylene and silicone passive water samplers. Chemosphere 2002, 46, (8), $1157-1161$.

22. Galassi, S.; Volta, P.; Calderoni, A.; Guzzella, L., Cycling pp'DDT and pp'DDE at a watershed scale: the case of Lago Maggiore (Italy). Journal of Limnology 2006, 65, (2), 100-106. 23. Lohmann, R.; MacFarlane, J. K.; Gschwend, P. M., Importance of black carbon to sorption of native PAHs, PCBs, and PCDDs in Boston and New York, Harbor sediments. Environ Sci Technol 2005, 39, (1), 141-148.

24. Fernandez, L. A.; MacFarlane, J. K.; Tcaciuc, A. P.; Gschwend, P. M., Measurement of freely dissolved PAH concentrations in sediment beds using passive sampling with low-density polyethylene strips. Environ Sci Technol 2009, 43, (5), 1430-1436.

25. Tcaciuc, A. P.; Apell, J. N.; Gschwend, P. M., Modeling the transport of organic chemicals between polyetheylene and water in infinite and finite bath conditions. In 2015 .

26. Hale, S. E.; Martin, T. J.; Goss, K.-U.; Arp, H. P. H.; Werner, D., Partitioning of organochlorine pesticides from water to polyethylene passive samplers. Environ Pollut 2010, $158,(7), 2511-2517$.

27. Gerstl, Z.; Mingelgrin, U., Sorption of organic substances by soils and sediments. $J$ Environ Sci Heal B 1984, 19, (3), 297-312.

28. Leatherbarrow, J. E., N. David, B.K. Greenfield, J.J. Oram and J.A. Davis., Organochlorine pesticide fate in San Francisco Bay. In RMP Technical Report: SFEIContribution \#433., San Francisco Estuary Institute, Oakland, CA.: 2006.

29. ATSDR Toxicological Profile for DDT, DDE and DDD; US Department of Health and Human Services: 2002; pp 218-219. http://www.atsdr.cdc.gov/ToxProfiles/tp35.pdf

30. Putyrskaya, V.; Klemt, E.; Röllin, S., Migration of 137Cs in tributaries, lake water and sediment of Lago Maggiore (Italy, Switzerland) - analysis and comparison with Lago di Lugano and other lakes. J Environ Radioactiv 2009, 100, (1), 35-48.

31. Pirnie, E. F.; Talley, J. W.; Hundal, L. S., Abiotic transformation of DDT in aqueous solutions. Chemosphere 2006, 65, (9), 1576-1582.

32. Meyer, B. N.; Lam, C.; Moore, S.; Jones, R. L., Laboratory degradation rates of 11 pyrethroids under aerobic and anaerobic conditions. J Agric Food Chem 2013, 61, (20), 4702-8. 


\section{Supplemental Information}

\section{Contents}

1. Derivation of Laplace solution for reaction diffusion model.

2. Additional experimental detail.

\section{Tables}

Table S1. PRC concentrations (ng/g PE) in PE used in the field and in laboratory incubations. 


\section{Derivation of Laplace solution for reaction diffusion model.}

The general procedure for deriving Laplace domain solutions for the transfer between polymer membranes and various media has been described elsewhere, and we follow the same steps here.

Solution for PRCs. The governing equation are repeated here from the main text:

Governing equations:

$\frac{\partial C_{P E}}{d t}=D_{P E} \frac{\partial^{2} C_{P E}}{\partial x^{2}} \quad$ for $-L<x<L$

$\frac{\partial C_{S E D}}{d t}=D_{S E D} \frac{\partial^{2} C_{S E D}}{\partial x^{2}}-k_{r} C_{S E D} \quad$ for $L<x<L+\delta$ and $-\delta-L<x<-L$

and in addition, we write the following initial conditions:

$C_{P E}=C_{P E}^{0}$ for $-L<x<L$

$C_{S E D}=0$ for $x<-L$ and $x>L$

and

boundary

$\frac{\partial C_{P E}}{\partial x}=0 \quad$ at $x=0$

$C_{P E}=K_{P E S E D} C_{S E D} \quad$ at $x=L$ and $x=-L$

$D_{P E} \frac{\partial C_{P E}}{\partial x}=D_{S E D} \frac{C_{S E D}}{\partial x} \quad$ at $x=L$ and $x=-L$

$C_{S E D}=0 \quad$ at $x=\infty$ and $X=-\infty$

The first step in deriving the solution is to transform the equations and boundary conditions in non dimensional units. $\bar{C}, X, T$ are now unit-less variables for concentration, distance and time:

$$
\begin{aligned}
& \overline{C_{P E}}=\frac{C_{P E}}{C_{P E}^{0}} \text { and } \overline{C_{S E D}}=C_{S E D} \frac{K_{P E S E D}}{C_{P E}^{0}} \\
& X=\frac{x}{L} \text { and } T=t * \frac{D_{P E}}{L^{2}}
\end{aligned}
$$

where $\mathrm{L}$ is the half thickness of the polymer, $D_{P E}$ is the diffusivity in the PE and $K_{P E S E D}$ is the PE-sediment partition coefficient. In non-dimensional terms the initial conditions for $\mathrm{T}=0$ for a PRC diffusing out of PE become:

$$
\begin{aligned}
& \overline{C_{P E}}=1 \text { for }-1<X<1 \\
& \overline{C_{W}}=0 \text { for } X<-1 \text { and } X>1
\end{aligned}
$$

Similarly, at $\mathrm{T}>0$, we have the following boundary conditions in non dimensional units:

- No flux at the center of sampler: $\quad \frac{\partial \overline{C_{P E}}}{\partial T}=0$ at $X=0$;

- Chemical equilibrium at sampler/sediment interface: $\overline{C_{P E}}=\overline{C_{S E D}}$ at $X=1$ and $X=-1$ (14)

- No accumulation of mass at sampler-sediment interface 
$\frac{\partial \overline{C_{P E}}}{\partial X}=\frac{\psi}{K_{P E S E D}} \frac{\partial \overline{C_{S E D}}}{\partial X}$ at $X=1$ and $X=-1$, where $\psi=\frac{D_{S E D}}{D_{P E}}$

- At large distances away from the sampler, concentration equals to that of sediment, which is 0 for a PRC: $\overline{C_{S E D}}=0$ at $X=\infty$ and $X=-\infty$

Lastly, we perform the same change of variables for the governing equations:

$$
\begin{aligned}
& \frac{\partial^{2} \overline{C_{P E}}}{\partial X^{2}}=\frac{\partial \overline{C_{P E}}}{\frac{\partial T}{X_{S}}} \\
& \frac{\partial^{2} \overline{C_{S E D}}}{\partial X^{2}}-\frac{\overline{k_{r}}}{\psi} C_{S E D}=\frac{1}{\psi} \frac{\partial \overline{C_{S E D}}}{\partial T}
\end{aligned}
$$

where $\overline{k_{r}}$ is the reaction rate $\mathrm{k}_{\mathrm{r}}$ normalized by $\mathrm{D}_{\mathrm{PE}} / \mathrm{L}^{2}$ By taking Laplace transforms of the governing equations, we translate the equations from time domain to complex s domain, and thus reduce the complexity of the differential equations. $\widehat{C_{P E}}$ and $\widehat{C_{W}}$ denote the Laplace domain expression of concentration. It follows that we now have the following governing equations and boundary conditions that are independent of the time variable:

$\circ$ governing equations:

$$
\begin{aligned}
& \frac{\partial^{2} \widehat{C_{P E}}}{\partial X^{2}}=s \widehat{C_{P E}}-1 ; \\
& \frac{\partial^{2} \widehat{C_{S E D}}}{\partial X^{2}}-\frac{\overline{k_{r}}}{\psi} \widehat{C_{S E D}}=\frac{s}{\psi} \widehat{C_{S E D}}
\end{aligned}
$$

○ boundary conditions:

$$
\begin{array}{rlrl}
\frac{\partial \widehat{C_{P E}}}{\partial X}=0 & & \text { at } X=0 \\
\widehat{C_{P E}} & =\widehat{C_{S E D}} & & \text { at } X=1 \\
\frac{\partial \widehat{C_{P E}}}{\partial X}=\frac{\psi}{K_{P E S E D}} \frac{\partial \widehat{C_{S E D}}}{\partial X} & & \text { at } X=1 \\
\frac{C_{S E D}}{\partial}=0 & \text { at } X=\infty
\end{array}
$$

The solutions found to satisfy both differential equations and the first and last boundary condition above:

$$
\begin{aligned}
& \widehat{C_{P E}}=A \cosh (X \sqrt{s})+\frac{1}{s} \\
& \widehat{C_{S E D}}=B e^{\left(-X \sqrt{\frac{s+\overline{k_{r}}}{\psi}}\right)}
\end{aligned}
$$

Using the second and third boundary conditions above, we can solve for A and B:

$$
\begin{aligned}
& A=\frac{1}{s} \frac{1}{\sqrt{\frac{s}{s+k_{r}} \mathrm{~K}_{\mathrm{PEW}} \sqrt{\psi}} \sinh (\sqrt{s})-\cosh (\sqrt{s})} \\
& B=-A \sinh (\sqrt{s}) \frac{K_{P E S E D}}{\sqrt{\psi}} \sqrt{\frac{s}{s+k_{r}}} e^{\left(\sqrt{\frac{s+\overline{k_{r}}}{\psi}}\right)}
\end{aligned}
$$

Since we are ultimately interested in the fraction of PRC which remains in the PE after a certain time, we can integrate the concentration in the PE across the thickness of the PE:

$$
\widehat{M_{P R C}}=\int_{0}^{1} \widehat{C_{P E}} d X
$$

After plugging in A, and simplifying we are left with:

$$
\widehat{M_{P R C}}=\frac{1}{s}-\frac{1}{s^{\frac{3}{2}}} \frac{1}{\frac{\mathrm{K}_{\mathrm{PEW}}}{\sqrt{\psi}} \sqrt{\frac{s}{s+k_{r}}}+\operatorname{coth}(\sqrt{s})}
$$

The expression cannot be inverted to time domain using inversion tables. Instead we use a MATLAB numerical inversion algorithm invlap. ${ }^{1}$ to find the corresponding mass of PRC left 
in the PE as function of time T. A sample code to perform this operation is provided in SI-2.

Solution for target compounds. For target analytes diffusing into the PE, the governing equations remain the same as for the transport of PRCs, but the initial conditions and boundary conditions are different, written here directly in non-dimensional terms:

- Initial conditions at $\mathrm{T}=0$ :

$$
\begin{aligned}
& \overline{C_{P E}}=0 \text { for }-1<X<1 \\
& \overline{C_{S E D}}=1 \text { for } X<-1 \text { and } X>1
\end{aligned}
$$

- Boundary conditions at $T>0$

$$
\begin{array}{ll}
\frac{\partial \overline{C_{P E}}}{\partial X}=0 & \text { at } X=0 \\
\overline{C_{P E}}=\overline{C_{S E D}} & \text { at } X=1 \text { and } X=-1 \\
\frac{\partial \overline{C_{P E}}}{\partial \hat{x}}=\frac{\psi}{K_{P E W}} \frac{\partial \overline{C_{S E D}}}{\partial X} & \text { at } X=1 \text { and } X=-1 \\
\frac{\partial \overline{C_{S E D}}}{\partial T}=-\overline{\mathrm{k}}_{\mathrm{r}} T & \text { at } X=\infty \text { and } X=-\infty
\end{array}
$$

We note that as opposed to the PRC case, the concentration is now normalized by $C_{S E D}^{0}$, the initial concentration of the analyte in the sediment. Unlike a diffusion case, though, we can no longer assume that the sediment concentration stays constant over time. Instead, at a distance far away from the sampler the concentration in the sediment is only affect by the intrinsic rate constant.

The governing equations in Laplace domain are slightly different due to the change in the initial conditions:

$$
\begin{aligned}
& \frac{\partial^{2} \overline{C_{P E}}}{\partial X^{2}}=s \widehat{C_{P E}} ; \\
& \frac{\partial^{2} \overline{C_{S E D}}}{\partial X^{2}}=\frac{s+\overline{k_{r}}}{\psi} \overline{C_{S E D}}-\frac{1}{\psi} ;
\end{aligned}
$$

And the boundary conditions in Laplace domain are also slightly different:

$$
\begin{array}{rlrl}
\frac{\partial \widehat{C_{P E}}}{\partial X}=0 & & \text { at } X=0 \\
\widehat{C_{P E}} & =\widehat{C_{S E D}} & & \text { at } X=1 \\
\frac{\partial \widehat{C_{P E}}}{\partial \hat{x}}=\frac{\psi}{K_{P E S E D}} \frac{\partial \widehat{C_{S E D}}}{\partial X} & & \text { at } X=1 \\
\widehat{C_{S E D}}=\frac{1}{s+\overline{k_{r}}} & & \text { at } X=\infty
\end{array}
$$

The solutions that satisfy the governing equations, and the first and last boundary conditions, take the form:

$$
\begin{aligned}
& \widehat{C_{P E}}=D \cosh (X \sqrt{s}) \\
& \widehat{C_{S E D}}=E e^{\left(-X \sqrt{\frac{s+k_{r}}{\psi}}\right)}+\frac{1}{s+\overline{k_{r}}}
\end{aligned}
$$

After solving for $\mathrm{D}$ and $\mathrm{E}$ using the second and third boundary condition, we get:

$$
\begin{aligned}
& D=\frac{1}{s+\overline{k_{r}}} \frac{1}{\cosh (\sqrt{s})+\frac{\mathrm{K}_{\mathrm{PEW}}}{\sqrt{\psi}} \sqrt{\frac{s}{\left(s+\overline{k_{r}}\right)}} \sinh (\sqrt{s})} \\
& E=-D \frac{\mathrm{K}_{\mathrm{PEW}}}{\sqrt{\psi}} \sqrt{\frac{s}{\left(s+\overline{k_{r}}\right)}} \sinh (\sqrt{s}) e^{\left(\sqrt{\frac{s+k_{r}}{\psi}}\right)}
\end{aligned}
$$


Lastly, $\overline{M_{\text {target }}}$ can now be calculated as:

$$
\begin{aligned}
& \widehat{M_{\text {target }}}=\int_{0}^{1} \widehat{C_{P E}} d X \\
& \widehat{M_{\text {target }}}=\frac{1}{\sqrt{s}\left(s+k_{r}\right)} \frac{1}{\operatorname{coth}(\sqrt{s})+\frac{K_{\text {PEW }}}{\sqrt{\psi} \sqrt{\frac{s}{s+k_{r}}}}}
\end{aligned}
$$

As expected the solution reduces for $\mathrm{k}_{\mathrm{r}}=0$ to that found by Fernandez et al. (2009) ${ }^{2}$ :

$$
\widehat{M_{\text {target }}}=\frac{1}{s^{\frac{3}{2}}} \frac{1}{\operatorname{coth}(\sqrt{s})+\frac{\mathrm{K}_{\mathrm{PEW}}}{\sqrt{\psi}}}
$$




\section{Additional experimental details}

Incubation of lake sediment with $d 84,4^{\prime}-D D T$. Field collected F2 sediment was used to determine if DDT could degrade in the laboratory conditions used typically for ex-situ sampling (i.e. tumbling). Nine $50 \mathrm{~mL}$ combusted pear shaped flasks were spiked with $1 \mu \mathrm{g}$ ${ }^{13} \mathrm{C}$ PCB 153 and $5 \mu \mathrm{g} \mathrm{d} 8$ 4,4'-DDT ( $25 \mu \mathrm{L}$ of a solution in hexane). To each flask about $20 \mathrm{~g}$ wet sediment and $40 \mathrm{~mL}$ of Milli- $\mathrm{Q}$ water were added. The flasks were then put on a tumbler and removed after 4 (duplicate), 11, 14, 22, 30 (duplicate), 50, 60 days. The sediment slurry was poured off into an amber jar and frozen until freeze-dried, and the walls of the flask were washed with a mixture of methanol and DCM (to check that the PCB and DDT spike had mixed into the sediment slurry, as opposed to remaining on the walls). Aliquots of $1 \mathrm{~g}$ of the freeze-dried sediment were extracted using the procedure described previously for analysis of sediments.

Clarus GCMS analysis of d8 4,4'-DDT spiked sediments. The analysis was performed with a programmable inlet and a DB-XLB column (30 m, $0.25 \mathrm{~mm}$ ID, $1 \mu \mathrm{m}$ film thickness, Agilent Technologies, Lexington, MA). Injections of $1 \mu \mathrm{L}$ were done at $70{ }^{\circ} \mathrm{C}$, in splitless mode, and the inlet temperature was ramped to $275^{\circ} \mathrm{C}$ at $200^{\circ} \mathrm{C} / \mathrm{min}$. The split valve $(20: 1$ split ratio) was open after $1.5 \mathrm{~min}$. The column temperature was held at $75{ }^{\circ} \mathrm{C}$ for $1.5 \mathrm{~min}$, then ramped to 200 at $25{ }^{\circ} \mathrm{C} / \mathrm{min}$, and to $275^{\circ} \mathrm{C}$ at $4{ }^{\circ} \mathrm{C} / \mathrm{min}$. Helium was used as a carrier gas at $2 \mathrm{~mL} / \mathrm{min}$ and the MS source temperature was held at $250^{\circ} \mathrm{C}$. For analysis of DDT in sediment samples, the inlet liner (deactivated liner, without glass wool, Restek, Bellefonte, PA) was periodically removed and cleaned, to prevent degradation of the DDT. Degradation was monitored by the loss of signal of ${ }^{13} \mathrm{C} 4,4$ '-DDT added to each extract as an injection standard.

Results of $d_{8} 4,4$-DDT sediment incubation experiments. We observed a marked decrease in DDT concentration compared to the spiked amount, but the data were not suitable for evaluating a reaction rate. Although more than $80 \%$ of the spiked DDT was lost in all sampled flasks, there was no correlation between DDT concentration and incubation time. However, we are fairly confident that the observed loss of DDT was not due to an experimental artifact, because both the PCB and DDT spikes were loaded into the tumbling flaks from the same solution (thus at a known and constant mass ratio to each other of 1:5 PCB:DDT). It is unlikely that DDT could have crystalized in the sediment, (which would 
have lead to a heterogeneous concentration), since the levels at which DDT was spiked were fairly low ( $<500 \mathrm{ng} / \mathrm{g}$ sediment). Even if crystals would have formed when the chemicals were spiked into the sediment, DDT and PCB 153 have similar chemical properties (MW 360 and 354, Kow $10^{6.9}$ and $10^{6.9}$, melting points of 103 and $108{ }^{\circ} \mathrm{C}$, respectively), and thus should have dissolved at the same rate. The loss to the walls of DDT was also negligible. 
Table S1 PRC concentrations (ng/g PE) in PE used in the field and in laboratory incubations, as well as limits of detection (LOD, ng/g) determined from the sum of average peak area and three standard deviations of solvent blank runs and assuming mass of PE extracted given in parentheses.

\begin{tabular}{l|cccc|cccc} 
Compound & Field PE & SD & $\%$ & $\begin{array}{c}\text { LOD } \\
(\mathbf{6 0} \mathbf{~ m g )}\end{array}$ & Lab PE & SD & $\%$ & $\begin{array}{c}\text { LOD } \\
\mathbf{( 1 5} \mathbf{~ m g})\end{array}$ \\
\hline \hline${ }^{13}$ C PCB 28 & 153 & 4 & $3 \%$ & 0.1 & 266 & 35 & $13 \%$ & 0.5 \\
${ }^{13}$ C PCB 47 & 152 & 2 & $1 \%$ & 0.1 & 243 & 4 & $2 \%$ & 0.7 \\
${ }^{13}$ C 4,4'-DDE & 192 & 4 & $2 \%$ & 0.1 & 298 & 13 & $4 \%$ & 1.2 \\
${ }^{13}$ C PCB 111 & 171 & 10 & $6 \%$ & 0.1 & 230 & 26 & $11 \%$ & 0.7 \\
${ }^{13}$ C 2,4'-DDT & 246 & 6 & $2 \%$ & 0.2 & 380 & 31 & $8 \%$ & 1.1 \\
${ }^{13}$ C 4,4'-DDD & 143 & 2 & $1 \%$ & 0.1 & 284 & 12 & $4 \%$ & 0.5 \\
${ }^{13}$ C PCB 153 & 115 & 3 & $2 \%$ & 0.1 & 198 & 30 & $15 \%$ & 0.9
\end{tabular}

aThe LOD for PE concentrations translates into a LOD of detection for aqueous concentrations of 0.1 to $1 \mathrm{pg} / \mathrm{L}$ (for $K_{P E W}$ ranging from $10^{5}$ to $10^{6}$ ). 


\section{References}

1. Hollenbeck, K. J. INVLAP.M: A matlab function for numerical inversion of Laplace transforms by the de Hoog algorithm http://www.isva.dtu.dk/staff/karl/invlap.htm 2. Fernandez, L. A.; Harvey, C. F.; Gschwend, P. M., Using Performance Reference Compounds in Polyethylene Passive Samplers to Deduce Sediment Porewater Concentrations for Numerous Target Chemicals. Environ Sci Technol 2009, 43, (23), 88888894. 



\section{Chapter 7}

Conclusions 
The first goal of this thesis was to expand the use of passive sampler use towards understanding the HOC toxicity and bioaccumulation potential of mixtures. In Chapters 2 and 3 , methods were developed for estimating passive sampler-water, as well as membrane lipid-water partition coefficients, which add to existing GC $\times$ GC-based methods for calculation of other environmentally relevant physicochemical properties (e.g. vapor pressure, octanol-water partition coefficients and others). ${ }^{1,2}$ The GC $\times$ GC-based model allowed estimations of samplerwater and lipid-water partition coefficients to within 0.5 and $0.3 \log$ units (factor of 3 and factor of 2), respectively. In Chapters 3 and 5 we illustrated the application of the GC $\times$ GC-based partition coefficients, for evaluating bioaccumulation and toxicity of mixtures.

Using $\mathrm{GC} \times \mathrm{GC}$ retention times, we evaluated the relative partitioning between $\mathrm{PE}$ and lipids for a range of HOCs and found that the partition coefficients for lipid-water and PE-water are similar (typically within one log unit of each other). However, depending on the polarity of the chemical, the partitioning favored the lipid or PE phase. Aliphatic compounds such as alkanes will preferentially accumulate in the PE by a factor of 10 , whereas more polar chemicals, like PAHs, will accumulate to a greater extent in the lipids (factor of 5-6). This trend was supported by a partitioning experiment in Chapter 5 . Thus, even though passive sampling with polymer membranes is often referred to as biomimetic due the affinity of both the membranes and lipids for hydrophobic chemicals, ${ }^{3}$ the load and distribution of HOCs in the lipids is not expected to be the same as that measured in the passive samplers (at least for polyethylene).

The combination of passive sampling and GC $\times$ GC-based partition coefficients provided good estimates for the bioaccumulation in the polychaete Nereis virens of PCBs, but not for other compounds like PAHs, which can be metabolized by this species or its gut microflora. However, the GC $\times$ GC-based estimates of bioaccumulation were still useful because they served as (1) upper limit of tissue concentration as well as (2) a starting point for identification of situations where metabolism is affecting the tissue concentrations. Recent studies have shown that the concentration of HOCs in food webs is bound at the upper limit by the chemical activity in sediments. ${ }^{4}$ The combination of the $\mathrm{GC} \times \mathrm{GC}$ and passive sampling can help us estimate the activity for legacy pollutants but also for other nonpolar contaminants of emerging concern.

The toxicity of HOC mixtures was consistent with estimations of lipid body burdens based on GC $\times$ GC-FID analysis. The baseline toxicity of PE equilibrated with contaminated sediments was much greater than one would have expected based solely on the concentration of the EPA 16 
PAHs and other priority pollutants in the sediments. While the aromatic fraction of the mixture appeared to contribute to the toxicity more than the saturated portion (more favorable thermodynamics and kinetics), the estimations of toxicity were much closer to observed values when the contributions of all HOCs present in PE were considered. Thus, risk assessment for baseline toxicity based on priority pollutants alone is not a good metric at sites where other aromatic compounds (e.g. methylated derivatives of PAH or heterocyclic aromatic compounds) are abundant. The procedures laid out in this thesis would enable either direct measurement of baseline toxicity (with passive dosing) or estimations of cumulative body burdens at equilibrium (with GC $\times$ GC). To our knowledge, this is the first study which attempted to measure the cumulative baseline toxicity effects due to, and at the respective chemical activity levels of, HOC mixtures in sediments.

The second goal of this thesis was to improve our understanding of how passive samplers behave in the environment, which could aid in their implementation and facilitate novel applications. Starting with simple mathematical formulations of diffusion in the membrane and in the water (Chapter 4), we show that the transfer of hydrophobic compounds $\left(K_{O W}>10^{4}\right)$ between PE and water is mainly controlled by diffusion through the water boundary layer (WBL). This implies that future efforts at designing faster equilibrium samplers need to focus on reducing the water side resistance to transfer (by inducing flow or turbulence). Alternatively, since labeled compounds used as performance reference compounds (PRCs) can be a big part of the passive sampling costs, alternative applications without PRCs are worth considering for reducing sampling costs. Based on the mass transfer model, using polymers with slower diffusivities (such as polyoxymethylene) could lead to similar equilibration timescales (diffusivity in polymers $\sim 10^{-10}-10^{-11} \mathrm{~cm}^{2} / \mathrm{s}$, implying time to equilibrium of days to months) as we currently see for WBL controlled transfer using PE. ${ }^{5}$ Unlike boundary layer controlled transport, the membrane controlled transport is fully "determined" (with known values of partition coefficients and diffusivities we can calculate the exact fractional equilibration), and PRCs would not be needed. Another interesting model application (Chapter 5) was the use of polymer membranes to control chemical doses in toxicity tests. Lastly, since the model essentially describes the analytical solution for the transport through a boundary layer, it could be used in combination with tracer studies, to determine characteristic diffusive boundary layer 
thickness in various environments. Aqueous boundary layer thicknesses are in turn relevant for dissolution, nutrient uptake and other geochemical processes.

A common assumption in passive sampling studies that employ performance reference compounds (PRCs) to adjust for disequilibrium between samplers and the environment is that the exchange of PRCs and target chemicals is diffusion controlled and therefore symmetric. In Chapter 6 we showed that reactive processes can also affect the behavior of PRCs, and considerations of reactivity should be taken into account when interpreting the PRC loss (i.e. complete PRC loss may not correspond to complete equilibration of a target compound). Furthermore, using a reaction diffusion mass transfer model and PRC loss from in situ passive samplers deployed in sediments, we determined that the degradation rate of DDT in a freshwater lake sediment increased with depth into the sediment bed from $0.09 \mathrm{~d}^{-1}$ at $0-10 \mathrm{~cm}$ and $0.9 \mathrm{~d}^{-1}$ at $30-40 \mathrm{~cm}$. The degradation was confirmed by corresponding accumulation of the PRC-derived degradation product in the PE. The magnitude of reaction rates was in line with other measurements in the literature and with the complete transformation of DDT to DDE and DDD observed in the contaminated lake sediments. More research is needed though to assess the accuracy of the degradation rate calculated from PRC loss, as well as the processes that are causing this degradation (e.g. biotic or abiotic reductive dechlorination)

\section{Future work}

The findings and techniques presented in this thesis open the door to various further research opportunities. Passive dosing-based testing could be adapted towards investigations of other mixtures, and other toxicity modes. For example, 10 day tests with Daphnia pulex (commonly used for reproductive toxicity tests ${ }^{6}$ ) in combination with passive dosing, could be used to assess the effect of environmental mixtures on reproductive success. Using dosing sheets could also be helpful in long term tests where in the absence of buffering, the chemical concentration can be affected by introduction of food or water exchange. If the dosing sheets are equilibrated with environmental mixtures, the passive samplers can effectively be used not only to measure chemicals in the environment but also as "toxicity meters."

Another potential research avenue is the application of the reaction-diffusion model in Chapter 6 towards other chemicals susceptible to transformations. For example, pyrethroids, a class of insecticides found to be toxic to fish and birds, ${ }^{7}$ are susceptible to transformations in 
sediments on timescales of months to years. ${ }^{8}$ Measuring in situ degradation of these compounds could help us understand the conditions that impact the degradation (e.g., presence of particular microbes, redox potential) and the mechanisms of degradation. Information about environmental transformations can in turn aid in selection of pesticides by balancing effectiveness with potential harmful effects.

Some studies have suggested that the degradation of DDT is tightly coupled with the oxidation reduction potential in sediments and soils. ${ }^{9}$ Thus the observed loss of a reactive compound from PE (not necessarily DDT) could function as a "redox probe." Furthermore, if the observed DDT loss was due to reductive dechlorination, then PE in combination with PRCs could be used as in situ indicators of conditions favorable for reductive dechlorination.

While passive samplers are broadly considered to be biomimetic devices that accumulate HOCs similarly as the lipid compartments of biota, the $\mathrm{GC} \times \mathrm{GC}$ model presented in this thesis suggested that there is preferential accumulation of more polar compounds in lipids and preferential accumulation of more saturated chemicals in PE. This implication could be further investigated with $\mathrm{GC} \times \mathrm{GC}$ for other sampling polymers, which may be able to better mimic the partitioning into membrane lipids. 


\section{References}

1. Arey, J. S.; Nelson, R. K.; Xu, L.; Reddy, C. M., Using comprehensive two-dimensional gas chromatography retention indices to estimate environmental partitioning properties for a complete set of diesel fuel hydrocarbons. Anal Chem 2005, 77, (22), 7172-82.

2. Nabi, D.; Gros, J.; Dimitriou-Christidis, P.; Arey, J. S., Mapping environmental partitioning properties of nonpolar complex mixtures by use of GC x GC. Environ Sci Technol 2014, 48, (12), 6814-26.

3. Vrana, B.; Mills, G. A.; Allan, I. J.; Dominiak, E.; Svensson, K.; Knutsson, J.; Morrison, G.; Greenwood, R., Passive sampling techniques for monitoring pollutants in water. Trac Trend Anal Chem 2005, 24, (10), 845-868.

4. Jahnke, A.; MacLeod, M.; Wickstrom, H.; Mayer, P., Equilibrium sampling to determine the thermodynamic potential for bioaccumulation of persistent organic pollutants from sediment. Environ Sci Technol 2014, 48, (19), 11352-9.

5. Lohmann, R., Critical review of low-density polyethylene's partitioning and diffusion coefficients for trace organic contaminants and implications for its use as a passive sampler. Environ Sci Technol 2012, 46, (2), 606-18.

6. Stark, J. D.; Vargas, R. I., Toxicity and hazard assessment of fipronil to Daphnia pulex. Ecotox Environ Safe 2005, 62, (1), 11-16.

7. Thatheyus, A. J.; Selvam, A. D. G., Synthetic pyrethroids: toxicity and biodegradation. Appl Ecol Env Sci 2013, 1, (3), 33-36.

8. Meyer, B. N.; Lam, C.; Moore, S.; Jones, R. L., Laboratory degradation rates of 11 pyrethroids under aerobic and anaerobic conditions. J Agric Food Chem 2013, 61, (20), 4702-8.

9. Gambrell, R. P.; Reddy, C. N.; Collard, V.; Green, G.; Patrick, W. H., The recovery of DDT, kepone, and permethrin added to soil and sediment suspensions incubated under controlled redox potential and pH conditions. J Water Pollut Con F 1984, 56, (2), 174-182. 